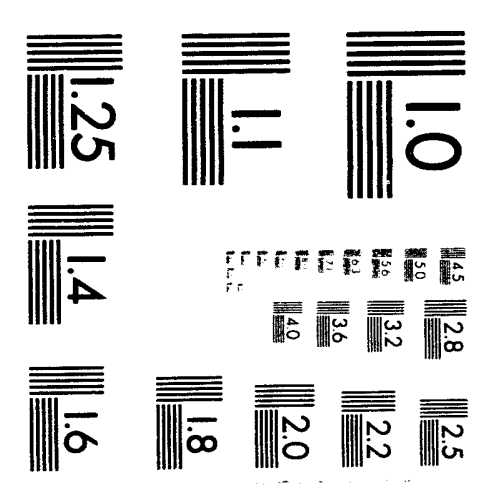



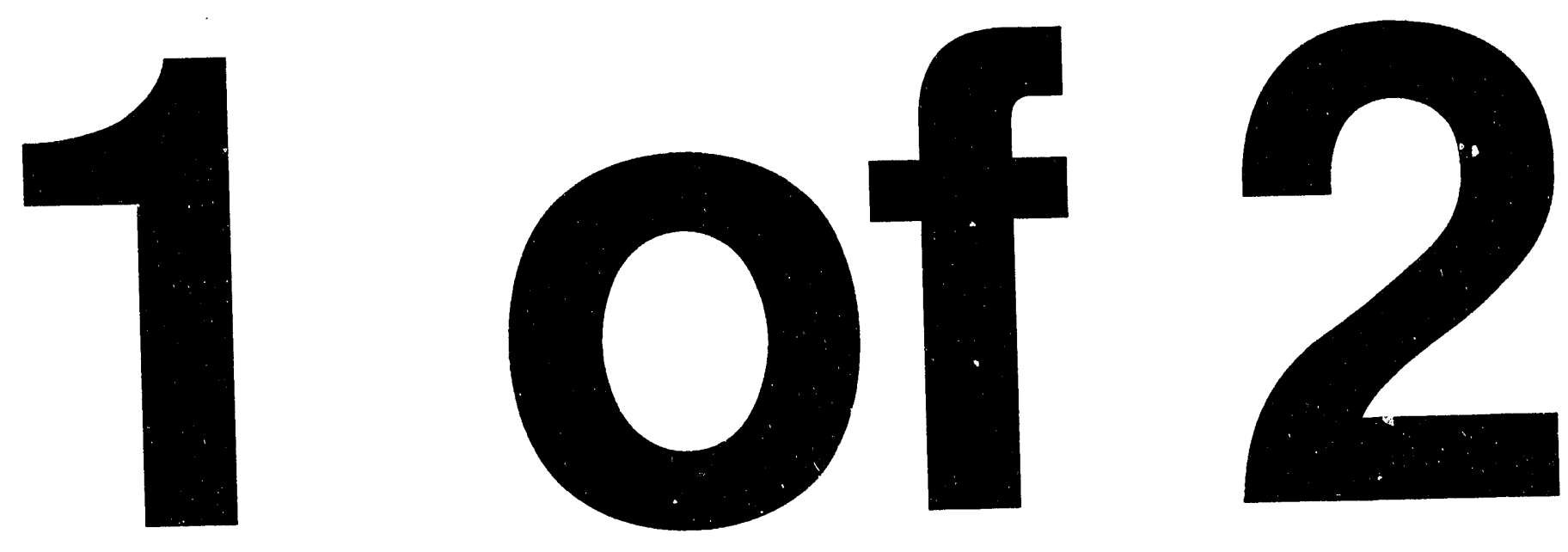


\title{
A Study of Diagnostic X-Ray Lines in Heliumlike Neon Using an Electron Beam Ion Trap
}

\author{
Bradford John Wargelin
}

(Ph.D. Thesis)

Manuscript date: October 1993

LAWRENCE LIVERMORE NATIONAL LABORATORY

University of California • Livermore, California 994551

\section{MASTER}


A Study of Diagnostic X-Ray Lines in Heliumlike Neon Using an Electron Beam Ion Trap

by

Bradford John Wargelin

B.S. (Massachusetts Institute of Technology) 1985

A dissertation submitted in partial satisfaction of the requirements for the degree of

Doctor of Philosophy

in

Physics

in the

GRADUATE DIVISION

of the

UNIVERSITY of CALIFORNIA at BERKELEY

Committee in charge:

Professor Steven M. Kahn, Chair

Professor Roger W. Falcone

Professor Alexei V. Filippenko 
The dissertation of Bradford John Wargelin is approved:
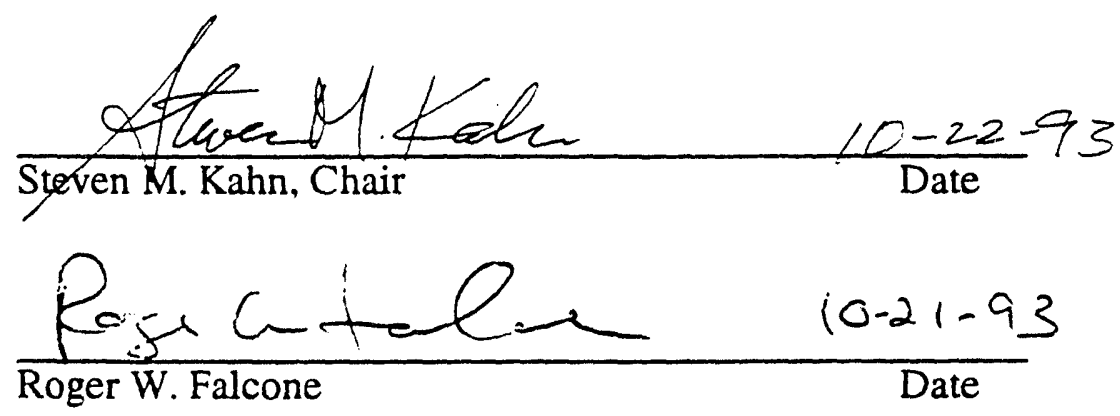

$\frac{\text { Alexei V. Filippents } \quad 10-22-73}{\text { Alexei V. Filippenko }}$

University of California at Berkeley

1993 
A Study of Diagnostic X-Ray Lines in Heliumlike Neon

Using an Electron Beam Ion Trap

by

Bradford John Wargelin

\begin{abstract}
Heliumlike ions play an extremely important role in X-ray astrophysics because of their emissivity and because the relative intensities of their emission lines can be used to infer physical characteristics of $\mathrm{X}$-ray emitting plasmas, including temperature, electron density, and ionization balance. Ir. Jer to properly apply these diagnostics, accurate atomic data are required, including cross sections for collisional excitation and ionization, radiative rates, and the wavelengths and strengths of satellite lines. Although theoretical atomic models have been created to estimate many of the rates and cross sections involved, very few experimental results are available for comparison with theoretical predictions.

This thesis describes an experimental study of heliumlike neon using an electron beam ion trap, a device specifically designed to study X-ray emission from highly charged ions. Using a low-energy $\mathrm{X}$-ray spectrometer designed and built for this experiment, electron impact excitation cross sections and dielectronic satellite strengths were measured for all significant $n=2 \rightarrow 1$ emission lines in He-like and Lilike Ne over a range of energy extending from well below the direct excitation threshold of the lines to over fourteen times the threshold energy. The cross section for innershell ionization of $\mathrm{Li}$-like $\mathrm{Ne}$, which excites the He-like forbidden line, was also measured. In addition, the radiative and collisional depopulation rates of the metastable $1 s^{2} s^{3} S_{1}$ state, which form the basis of the He-like Ne density diagnostic, were determined.

Experimental results were generally in agreement with theoretical predictions, although some significant differences were noted, particularly for the wavelengths and resonance strengths of dielectronic satellites. In addition, the excitation cross section for the intercombination line blend was found to be $15 \%$ higher than predicted by theory, while the cross section of the forbidden line was $25 \%$ higher. Results from the study of innershell ionization and excitation of Li-like Ne were used to distinguish between three widely varying predictions of the fluorescence yield of the primary $\mathrm{Li}$ like $K_{\alpha}$ line. The radiative lifetime measurement had a precision of nearly one percent and was found to be in excellent agreement with theoretical predictions.
\end{abstract}


A Study of Diagnostic X-Ray Lines in Heliumlike Neon Using an Electron Beam Ion Trap 


\section{Contents}

List of Figures $\ldots \ldots \ldots \ldots \ldots \ldots \ldots \ldots \ldots \ldots \ldots$

List of Tables . . . . . . . . . . . . . . . . . . . . . .

Acknowledgments $\ldots \ldots \ldots \ldots \ldots \ldots \ldots \ldots \ldots \ldots \ldots \ldots \ldots \ldots \ldots \ldots \ldots \ldots$

1 Introduction 1

1.1 Astrophysical X-Ray Sources . . . . . . . . . . . . . 2

1.2 Spectroscopic Line Diagnostics $\ldots \ldots \ldots \ldots \ldots \ldots \ldots \ldots \ldots$

1.3 Organization of Thesis $\ldots \ldots \ldots \ldots \ldots \ldots \ldots \ldots \ldots$

2 Heliumlike Ions $\quad 10$

2.1 Astrophysical Significance $\ldots \ldots \ldots \ldots \ldots \ldots \ldots \ldots \ldots$

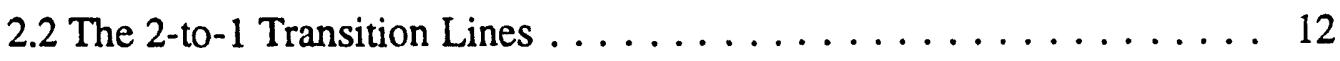

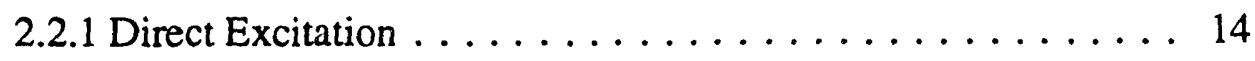

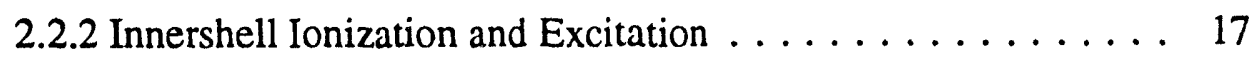

2.2.3 Recombination Mechanisms . . . . . . . . . . . . . 19

2.3 Density Diagnostics . . . . . . . . . . . . . . . . 24

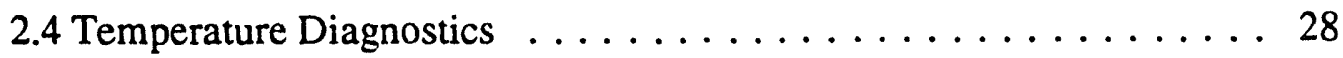

2.5 Ionization Balance Diagnostics $\ldots \ldots \ldots \ldots \ldots \ldots \ldots \ldots . \ldots \ldots$

2.6 Existing Data, Calculations, and Applications $\ldots \ldots \ldots \ldots \ldots . \ldots 32$

3 The Electron Beam Ion Trap $\quad 34$

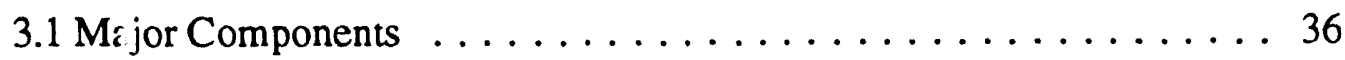

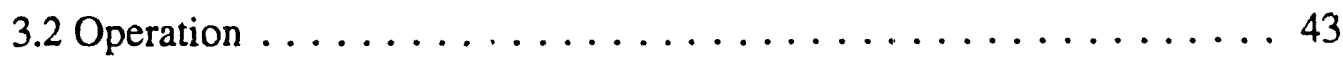

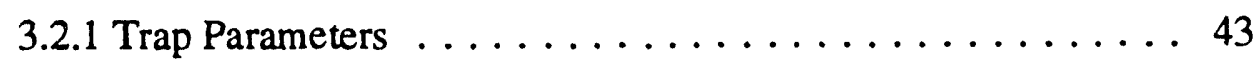

3.2.2 Control System and Relevent Timescales ......... 46

3.2.3 Data Acquisition Systems . . . . . . . . . . . . . 50

4 Soft X-Ray Crystal Spectrometer

4.1 Diffraction Crystals $\ldots \ldots \ldots \ldots \ldots \ldots \ldots \ldots \ldots \ldots$

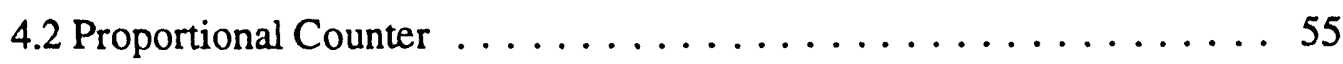

4.3 General Layout $\ldots \ldots \ldots \ldots \ldots \ldots \ldots \ldots \ldots \ldots \ldots$ 
5 Experimental Procedure 63

5.1 Desired Measurements . . . . . . . . . . . . . . . . 63

5.2 Low Energy Measurements . . . . . . . . . . . . . . . 65

5.3 High Energy Measurements . . . . . . . . . . . . . 69

6 Polarization $\quad 74$

6.1 Background and Theory . . . . . . . . . . . . . 74

6.2 Theoretical Calculation of Line Polarizations . . . . . . . . . 80

6.2 .1 Cascades . . . . . . . . . . . . . . . 82

6.2.2 Collisional Transfer from the Metastable ${ }^{3} S_{1}$ State . . . . . . 87

6.2.3 Effect of Transverse Electron Velocity . . . . . . . . . . 89

6.3 Polarization Measurements . . . . . . . . . . . . . 92

6.3.1 Experimental Methods . . . . . . . . . . . . . 93

6.3 .2 Polarization at $1 \mathrm{keV} \ldots \ldots \ldots \ldots \ldots$

6.4 Intensity Correction Factors $\ldots \ldots \ldots \ldots 1$

7 Radiative Lifetime of the ${ }^{3} S_{1}$ State $\quad 105$

8 Analysis and Results $\quad 115$

8.1 Dielectronic Recombination Satellite Lines . . . . . . . . . . . 115

8.1.1 Fluorescence Yields . . . . . . . . . . . . . . . . 118

8.1.2 Theoretical Resonance Strengths . . . . . . . . . . . . 120

8.1.3 Experimental Measurement of KLL Resonance Strengths . . . 121

8.2 Low Energy Data . . . . . . . . . . . . . . . . . . . . . . . . . 129

8.2.1 Near-Threshold Line Intensities . . . . . . . . . . . . . . 130

8.2.2 Determination of Cross Sections and Resonance Strengths . . . 139

8.3 High Energy Data . . . . . . . . . . . . . . . . . . . 144

8.3.1 Excitation of $K_{\alpha}$ Lines at High Energies . . . . . . . . 145

8.3.2 Data Analysis and Fit Results . . . . . . . . . . . 148

8.3.3 Normalization of $\sigma_{w}(\mathrm{E}) \ldots \ldots \ldots \ldots \ldots \ldots$

8.4 Application to Temperature Diagnostic . . . . . . . . . 159

9 Concluding Remarks 163

9.1 Summary of Results . . . . . . . . . . . . . . . 163

9.2 Future Work . . . . . . . . . . . . . . . . 165

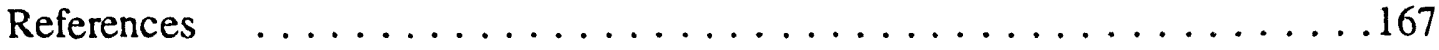




\section{List of Figures}

2.1 Fractional Ion Abundances Versus Temperature . . . . . . . . . 11

2.2 He-like Ion Energy Level Diagram $\ldots \ldots \ldots \ldots \ldots \ldots \ldots \ldots \ldots$

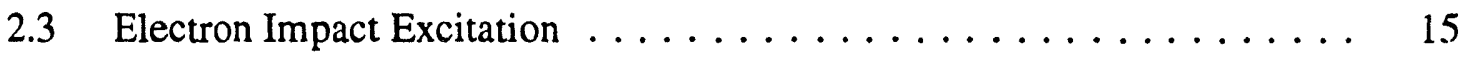

2.4 Innershell Ionization of a Li-like Ion $\ldots \ldots \ldots \ldots \ldots \ldots \ldots \ldots$

2.5 Spectrum of He-like Neon . . . . . . . . . . . . . . . 20

2.6 Recombination Mechanisms . . . . . . . . . . . . . 22

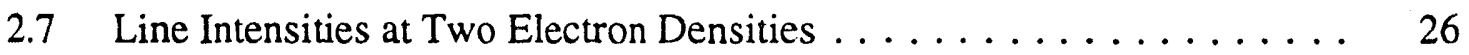

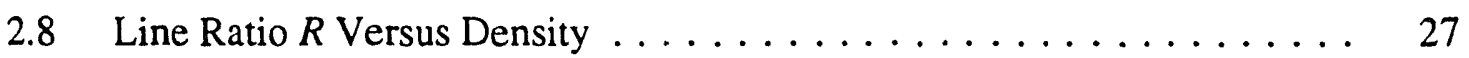

2.9 Electron Impact Excitation Cross Sections . . . . . . . . . . . . . . . . . . 29

2.10 Line Ratio $G$ Versus Temperature $\ldots \ldots \ldots \ldots \ldots \ldots \ldots \ldots \ldots$

$3.1 \quad$ Electron Beam Ion Trap $\ldots \ldots \ldots \ldots \ldots \ldots \ldots \ldots \ldots \ldots \ldots$

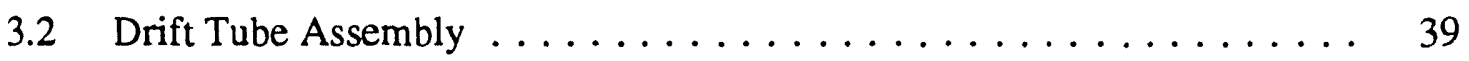

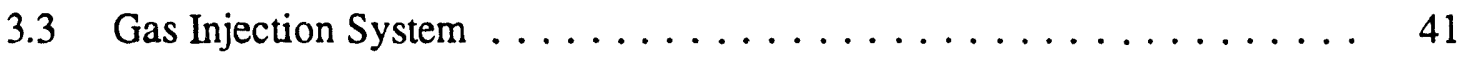

3.4 Example Timing Pattern for EBIT Control Program . . . . . . . . 47

4.1 Soft X-ray Flat Crystal Spectrometer $\ldots \ldots \ldots \ldots \ldots \ldots \ldots \ldots$

$4.2 \mathrm{X}$-ray Transmission of Various Window Materials $\ldots \ldots \ldots \ldots \ldots . \ldots 7$

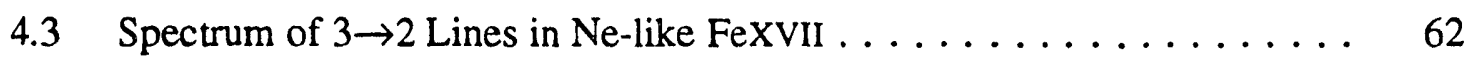

5.1 Timing Patterns for Electron Beam Energy Sweeps . . . . . . . . . 70

6.1 X-ray Emission Geometry . . . . . . . . . . . . . . . . 77

6.2 Theoretical Polarization of He-like $\mathrm{Fe} K_{\alpha}$ Lines $\ldots \ldots \ldots \ldots \ldots . \quad 81$

6.3 Theoretical Polarizations of He-like Ne $K_{\alpha}$ Lines with Cascades . . . . . 86

6.4 Theoretical Polarizations after Including Effects of Collisional Transfer, Transverse Electron Velocity, and Beam Energy Spread ........ 91

6.5 Spectra Obtained at $1000 \mathrm{eV}$ with Mica and TAP Crystals ........ 96

7.1 Spectra of He-like Neon Above and Below Threshold . . . . . . . . 107

7.2 Line Intensities as a Function of Time . . . . . . . . . . . . 109

7.3 Experimental Versus Theoretical Lifetimes for $z \ldots \ldots \ldots \ldots \ldots . \ldots 114$ 
8.1 Low-Energy Spectra Taken with Event Mode System . . . . . . . . . . 117

8.2 Energy Windows Used to Determine DR Resonance Strengths . . . . . . 123

8.3 Experimental and Theoretical KLL Spectra . . . . . . . . . . . . . 125

8.4 Event Mode Spectra Between 750 and $1200 \mathrm{eV} \ldots \ldots \ldots \ldots$

8.5 Experimental and Theoretical Cross Sections for $w \ldots \ldots \ldots \ldots$

8.6 Cross Sections for $x y \ldots \ldots \ldots \ldots \ldots \ldots \ldots \ldots \ldots$

8.7 Cross Sections for $z \ldots \ldots \ldots \ldots \ldots \ldots \ldots \ldots$

8.8 Cross Sections for $q r \ldots \ldots \ldots \ldots \ldots \ldots \ldots \ldots \ldots \ldots$

8.9 High Energy Data for $z$ and $q r \ldots \ldots \ldots \ldots \ldots \ldots \ldots$

8.10 High Energy Data for $x y$ and $z \ldots \ldots \ldots \ldots \ldots \ldots 15 \ldots \ldots \ldots$

8.11 Relative Cross Sections Derived from High Energy Data . . . . . . . . . 154

8.12 Energy Dependence of $\sigma_{w} \ldots \ldots \ldots \ldots \ldots \ldots \ldots$

8.13 Experimental and Theoretical Temperature Dependence of $G \ldots \ldots 16$ 


\section{List of Tables}

5.1 Atomic Processes Relevant to He-like Neon $K_{\alpha}$ Emission . . . . . . . . 66

6.1 Branching Ratios . . . . . . . . . . . . . . . . . 84

6.2 Theoretical and Experimental Line Polarizations . . . . . . . . . . . . 100

7.1 Results of Lifetime Measurements and Associated Uncertainties . . . . . 112

8.1 Atomic Data for $K L L$ Satellites in Li-like $\mathrm{Ne}^{7+} \ldots \ldots \ldots \ldots \ldots \ldots$

8.2 Atomic Data for $K L L$ Satellites in Be-like $\mathrm{Ne}^{6+} \ldots \ldots \ldots \ldots$

8.3 High Order Resonances . . . . . . . . . . . . . . . . . . 141 


\section{Acknowledgments}

This thesis would not have been possible without the guidance and support of many people, especially my thesis advisor, Steve Kahn, and my project advisor at LLNL, Peter Beiersdorfer. Steve has been a driving force in the field of "laboratory strophysics," being one of the first people to recognize the urgent need for accurate experimental data on the atomic physics of X-ray emission, so that high-resolution $\mathrm{X}$-ray spectra from upcoming satellite missions can be fully exploited. His efforts over the past several years have been rewarded by a growing awareness in the X-ray astrophysics community of the need for better atomic data, as evidenced by increased NASA funding for theoretical and experimental research on the frontier of astrophysics and atomic physics, including this thesis research.

The results presented here would not have been possible without the guidance of Peter Beiersdorfer. His expertise in atomic physics and the science of EBIT was invaluable for this project, and his detailed review of this thesis as it was being written challenged me to understand many issues that might have gone uninvestigated, resulting in a deeper understanding of atomic physics that should serve me very well in the years ahead. My only regret is that time was too short to fully explore the many ideas and suggestions he provided during my tenure as a graduate student.

Many other members of the EBIT team at Livermore deserve thanks, including Dave Knapp, who created most of the EBIT analysis software and the Event Mode data acquisition system, and Dan Nelson and Ed Magee, who helped build the Bradley Fighting Spectrometer and kept EBIT up and running. Thanks also go to the Livermore theorists who supplied cross section calculations and helpful advice, namely, Kennedy Reed, Al Osterheld, and Mau Chen.

I am especially indebted to Ed Kellogg and the other AXAF project directors at SAO for their patience and support as this thesis was finished, and to Roger Falcone and Alex Filippenko for their comments and suggestions. I also owe thanks to many friends at Berkeley who helped me with their advice, friendship, encouragement, and commiseration. In particular, I would like to thank Bill Craig, Fiona Harrison, Duane Liedahl, Frits Paerels, and Ron Wurtz.

Finally, I wish to thank my family for their support, encouragement, and love. I would never have reached this point without them. I am forever grateful to my wife, Peggy, who deserves as much credit for the completion of this thesis as anyone. 


\section{Chapter 1}

\section{Introduction}

This thesis presents the results of an experimental investigation of heliumlike and lithiumlike neon iois using an electron beam ion trap. The excitation cross sections of the four primary $K_{\alpha}(n=2 \rightarrow 1)$ lines and blends, specifically the He-like resonance line and its $\mathrm{Li}$-like collisional satellites, the He-like intercombination line blend, and the He-like forbidden line (which is also excited by innershell ionization of Li-like neon), were measured as functions of energy from below their excitation thresholds to approximately 14 times the threshold energy. The cross section for collisional depopulation of the metastable $1 s 2 s^{3} S_{1}$ level was also studied as a function of energy, along with the radiative lifetime of that level. The purpose of these measurements is to provide reliable experimental data for application in plasma diagnostics. The specific diagnostics discussed here can be used to determine temperature, density, ionization balance, and the degree of ionization nonequilibrium.

The need for these atomic data is driven by the impending launch of two X-ray telescope satellite missions which will produce abundant high-resolution spectral data from astrophysical X-ray sources. The Advanced X-ray Astrophysics Facility (AXAF) and X-ray Multi-Mirror Mission (XMM) are both slated for launch at the end of the decade and will have resolving powers $E / \Delta E$ ranging between approximately 100 and 1000. This resolution is roughly a factor of ten better than that of any nonsolar astrophysical X-ray spectra to date. Proper interpretation of these spectra will require a detailed and accurate understanding of the atomic physics involved, much of which will have to come from theoretical calculations Although there have been steady advances in theoretical atomic modeling codes, relatively little experimental data is 
available for comparison. Those data which do exist have often shown significant disagreement with theoretical predictions.

With this in mind, an increasing number of $\mathrm{X}$-ray astrophysicists have recognized that major efforts must be made now, in both theory and experiment, to obtain reliable, accurate, and comprehensive atomic data so that the wealth of high quality spectra provided by $A X A F$ and $X M M$ will not be wasted. This thesis is an attempt to contribute to that effort.

To help provide some context for this work and to give some indication of the wide range of physical conditions found in X-ray plasmas, a brief summary of astrophysical $\mathrm{X}$-ray sources is given in section 1.1 , followed by a short review of some important diagnostic methods in section 1.2. The chapter concludes with an overview of the organization of the rest of this thesis.

\subsection{Astrophysical X-Ray Sources}

$\mathrm{X}$-rays of astrophysical origin are emitted from hot gas with typical temperatures between $10^{5}$ and $10^{8} \mathrm{~K}$. As is true of all forms of electromagnetic radiation, X-rays can be emitted as discrete lines or as continuum emission. Line emission is generally the primary source of plasma cooling up to temperatures of a few times $10^{7} \mathrm{~K}$, beyond which most ions have been fully stripped of their electrons so that cooling is dominated by bremsstrahlung. Continuum emission is also generated by radiative recombination and 2-photon radiative decay. For high energy $X$-rays, Compton scattering can reprocess line emission into continua, and at large optical depths all radiation is reprocessed to produce blackbody emission.

Like $\mathrm{X}$-ray emission, astrophysical $\mathrm{X}$-ray sources can be divided into two general categories (with some overlap). In collisional plasmas, excitations are 
dominated by electron-ion collisions, resulting in ion excitation followed by spontaneous radiative decay. Most of the emission is from $K$-shell $(n \rightarrow 1)$ transitions in abundant elements from carbon $(Z=6)$ to sulfur $(Z=16)$, and $L$-shell $(n \rightarrow 2)$ emission from iron. Collisional plasmas are generally optically thin at all wavelengths, and if the plasma is also in ionization equilibrium it is referred to as being in "coronal" equilibrium.

$\mathrm{X}$-ray photoionized nebulae, or XPNs, are defined as plasmas in which radiation from a central X-ray continuum source (usually described by a power law) is reprocessed by an optically thick medium. The plasma is in that case overionized relative to its temperature (average electron kinetic energy) so that emission is dominated by radiative recombination continua and line emission from cascades. Depending on the source geometry and other physical parameters, absorption lines may also be prominent. In both types of X-ray source, a significant fraction of line emission is in the form of forbidden radiation from metastable states that are collisionally depopulated in laboratory plasmas. There are also, of course, intermediate cases such as in (portions of) accretion disks where both collisions and photoionization are important.

Examples of collisional plasma X-ray sources are early and late type stars, supernova remnants (SNRs), and hot gas surrounding galaxies and clusters of galaxies. The X-ray luminosity of stellar coronae is typically less than about $10^{33}$ ergs/sec, but thousands of stellar coronal X-ray sources were detected by the Einstein $\mathrm{X}$-ray satellite [61]. For comparison, the Sun typically has $L_{x} \approx 2 \times 10^{27} \mathrm{ergs} / \mathrm{sec}$, but $L_{x}$ can be larger during flares. The generally accepted model is that stellar coronae in late type stars $\left(\mathrm{F}\right.$ to $\mathrm{M}$ ) are somehow heated to temperatures of $10^{6}-10^{7} \mathrm{~K}$ by magnetic fields which are driven by some sort of dynamo in the convective layer of the star. This model is largely based on the strong correlation found between X-ray luminosity 
and stellar rotation velocities [67], and on comparisons with the Sun. Expected coronal densities are roughly $10^{9}-10^{10} \mathrm{~cm}^{-3}$, but can be up to $-10^{13} \mathrm{~cm}^{-3}$ in stellar flares, with the hot gas confined in magnetic loop structures.

$X$-rays have also been observed in early type stars ( $O$ and $B)$, but their method of generation is less clear. Early type stars do not have convection zones near the surface and are therefore expected to have relatively insignificant magnetic fields. Their strong radiation fields are believed, however, to drive powerful stellar winds. The two primary competing models for the source of X-ray emission are a hot corona at the base of the stellar wind [17], or shocks associated with gas blobs created by radiationdriven instabilities in the wind $[18,73]$.

Supernova remnants, apart from the occasional Crab-like nonthermal source at the center, can be approximated as expanding spherical shells of shocked gas. Timescales for ionization equilibrium are generally longer than the ages of young SNRs, so the $\mathrm{X}$-ray emitting plasma is underionized relative to its electron temperature. Most of the X-rays are emitted as lines, and detailed time-dependent models are required to adequately match observed spectra. Energy for production of the hot ionized gas that emits the X-rays comes from conversion of the bulk motion kinetic energy of the supernova ejecta (with typical velocities of $400 \mathrm{~km} / \mathrm{sec}$ ) into thermal energy as it expands into and shocks the surrounding interstellar medium.

Supernovae are believed to be a major source of the gas found in clusters of galaxies. This gas is extremely hot $\left(10^{7}-10^{8} \mathrm{~K}\right)$ and very tenuous $\left(-10^{-2} \mathrm{~cm}^{-3}\right)$, but because of the huge emitting volume of a cluster (of order a cubic megaparsec), X-ray emission can be detected at great distancss. The gas gains energy as it falls deeper into the gravitational well of the cluster, and since the gas is so diffuse, cooling times are on the order of the age of the universe. Near the center of a cluster, where densities are higher and the gas can therefore radiate more efficiently, cooling times 
are much shorter and the gas can depart from strict hydrostatic equilibrium, creating "cooling flows" [31a].

Cataclysmic variables (CVs), $\mathrm{X}$-ray binaries (XRBs), and active galactic nuclei (AGNs) are all accretion-powered sources that in many cases show signs of having XPNs. It should be noted, however, that X-ray emission from these sources is often dominated by continuum emission from the central photoionizing source. There can also be thermal emission and line emission from regions of the plasma that are collisionally excited. In all these cases, the energy source for X-ray production comes from the conversion of gravitational energy into thermal energy as gas accretes onto a central compact object. In CCVs, the accreting object is generally believed to be a white dwarf, in XRBs a neutron star or sometimes a black hole, and in AGNs a massive black hole (typically $10^{6}$ to $10^{9}$ solar masses).

\subsection{Spectroscopic Line Diagnostics}

During the earliest days of X-ray astronomy in the 1960s, proportional counters flown on rockets had a typical resolving power $E / \Delta E$ of order 1 or perhaps a few. By the 1970 s, improved counters with $E / \Delta E \approx 7$ were able to observe $K$-shell emission lines from iron at approximately $7 \mathrm{keV}$, as well as provide information on the general shape of X-ray source spectra. These low or moderate resolution spectra are usually fit with power law, blackbody, or thermal bremsstrahlung models which allow determination of approximate termperatures and luminosities. If the energy coverage extends below roughly $1 \mathrm{keV}$, the interstellar (or local) gas column density can also be estimated. Fe $K$-shell emission, which was first seen in the Cas A SNR and several KRBs, has received a great deal of attention because it is one of the very 
few spectroscopic features that can be resolved in low and moderate resolution spectra.

The Einstein observatory marked a significant improvement in sensitivity and spectral resolution. In addition to a proportional counter, Einstein had a solid state spectrometer with resolving power $E / \Delta E \approx 6(E / 1 \mathrm{keV})$, and two dispersive spectrometers, the Objective Grating Spectrometer, or OGS, with $E / \Delta E \approx 30$, and a Focal Plane Crystal Spectrometer (FPCS) with $E / \Delta E$ between 60 and 700, although most spectra had $E / \Delta E \approx 100$. The dispersive spectrometers, because of their relatively low efficiencies, were only practical because of Einstein's large collecting area and reflective focussing optics, and even then could only be used to observe the brightest sources.

OGS data from 22 bright Galactic X-ray sources, all of them accretion-powered and all but one XRBs, were recently analyzed by Vrtilek et al. [82], who found clear evidence for oxygen $K$-edge absorption in 10 of the sources, along with iron $L$ and neon $K$ edges in a few. X-ray line emission was detected in at least 11 of the sources and suggested that the emitting plasma spans a large range in temperature and ionization state. Neither purely collisional nor purely photoionized models could adequately explain the observed data, although rather crude photoionization models were used in that analysis. More recent and detailed work by Liedahl et al. [54, 55] suggests that photoionization models can, in fact, explain much of the data quite well.

A majority of the plasma diagnostics that have been proposed are most appropriate for collisional plasmas. A good review is given by Mewe [61]. More recently, Liedahl et al. $[54,55]$ have worked on Fe $L$-shell diagnostics for XPNs and found that spectra from photoionized plasmas are very different in appearance from collisionally excited spectra. 
In collisional plasmas, one of the most important parameters to determine is the temperature. The standard diagnostic method is to compare the intensities of two lines from the same ionization stage but with different excitation energies. Examples are the Lyman- $\alpha$ and Lyman- $\beta$ lines in H-like ions, or $K_{\alpha}$ and $K_{\beta}$ in He-like ions. If both lines cannot be observed at the same time, or instrumental effects are a concern, one can also measure the intensities of two lines that are very close in wavelength but have excitation cross sections with very different energy dependences. The best example is the ratio of the triplet and singlet $K_{\alpha}$ lines in He-like ions, which are discussed in detail in Chapter 2. The intensity ratios of resonance lines and some of their dielectronic satellites are also temperature sensitive because the excitation energy for a satellite lies significantly below the threshold for excitation of the parent line. This method is most easily applied to He-like iron which has relatively strong satellite lines.

Another important parameter is the electron density. As an example, if the density and luminosity of a source are known, then the emitting volume of the source can be determined, an important constraint for many theoretical models. Most density diagnostics are based on lines emitted from metastable states. The He-like triplet lines are particularly important and are discussed in Chapter 2. Iron $L$-shell emission is also density-sensitive because of the number of metastable "ground" states that lie just above the lowest energy ground state. At sufficiently high densities, these metastable states can be excited from the true ground state and then further excited (before the metastable state has time to radiatively decay) to levels which cannot be directly excited from the lowest ground state. 


\subsection{Organization of Thesis}

Chapter 2 discusses the theory behind the importance and utility of He-like ions as diagnostics of astrophysical plasma parameters. Explanations of all the processes which contribute to the excitation of He-like $K_{\alpha}$ emission are presented, including direct excitation, radiative and dielectronic recombination, and inner-shell ionization and excitation. It is then explained how the observed relative intensities of $K_{\alpha}$ lines provide information on the temperature, density, and ionization structure of X-ray emitting plasmas.

Chapters 3, 4, and 5 cover the equipment and procedures used in this investigation of He-like neon, beginning with a discussion of the electron beam ion trap. The main components and general operation methods are described, along with a brief summary of the data acquisition systems. Chapter 4 describes the low energy spectrometer that was designed and built specifically for this project, including the specially modified proportional counter used to detect the X-rays. Chapter 5 explains which measurements were desired and how the investigation was divided into two primary experiments: (1) a detailed, continuous-energy-coverage measurement at low energies and (2) a set of measurements at high energies in which the relative fractions of He-like and Li-like ions were varied in order to isolate the effects of direct excitation, innershell ionization, and collisional depopulation of the metastable state.

Chapter 6 combines a theoretical and experimental investigation of the linear polarization of $\mathrm{X}$-rays emitted from the ion trap. A model based on theoretical calculations of excitation cross sections is constructed to predict the effective cross section and polarization of each of the four primary $K_{\alpha}$ lines and blends. The effects of polarization on observed line intensities are determined for later application when 
analyzing data, and the results of an experimental measurement of line polarization are presented and compared with theory.

Chapter 7 presents the results of a separate experiment that measured the radiative lifetime of the ${ }^{3} S_{1}$ metastable state to an accuracy of $1.5 \%$. It also measured the effective electron density, a result that was used in analysis of other data obtained in this investigation.

Chapter 8 presents results from the analysis of data that are the primary focus of this thesis. Resonance strengths and wavelengths of dielectronic satellites are determined, along with excitation cross sections of all the $K_{\alpha}$ lines as a function of energy. Cross sections for inner-shell ionization of Li-like neon and collisional depopulation of the metastable state are also presented and compared with theory. A summary of results and suggestions for future work are listed in Chapter 9. 


\section{Chapter 2}

\section{Heliumlike Ions}

\subsection{Astrophysical Significance}

Heliumlike ions, defined as ions with only two remaining electrons, are arguably the most important isoelectronic species in X-ray astrophysics, for reasons that can be traced directly to their atomic structure. The $1 s^{2}$ configuration is a closed shell, so any additional electrons (in the $n=2$ shell) are much less tightly bound. For example, it takes only $207 \mathrm{eV}$ to remove one of the $2 s$ electrons from Be-like neon and $239 \mathrm{eV}$ to ionize $\mathrm{Li}$-like $\mathrm{Ne}$, but $1196 \mathrm{eV}$ to ionize He-like to $\mathrm{H}$-like and an additional 1363 $\mathrm{eV}$ to fully strip the nucleus. For this reason, He-like ions can exist over an extremely large temperature range, in fact, the largest range of any isoelectronic species (see Figure 2.1).

The characteristic teinperature for He-like ions depends on the element. After hydrogen and helium, which together account for $99.8 \%$ of the interstellar medium, the most astrophysically abundant elements are $\mathrm{C}, \mathrm{N}, \mathrm{O}, \mathrm{Ne}, \mathrm{Mg}, \mathrm{Si}, \mathrm{S}$, and $\mathrm{Fe}$, with oxygen being the most abundant of these "metals." Typical temperatures of X-ray emitting gas lie roughly between $10^{5}$ and $10^{8} \mathrm{~K}$, with He-like carbon most prominent at the low end while He-like iron dominates at high temperatures. Above $10^{8} \mathrm{~K}$ even Fe nuclei have been fully stripped of their electrons, and radiative cooling is dominated by thermal bremsstrahlung.

The characteristic line emission of He-like ions is known as $K$-shell emission and lies in the X-ray band for all the elements from $\mathrm{C}(\sim 300 \mathrm{eV})$ to $\mathrm{Fe}(\sim 6700 \mathrm{eV})$. $K_{\alpha}$ denotes $n=2$ to $n=1$ transitions, $K_{\beta}$ is $3-$ to-1, and so on. The numerical 


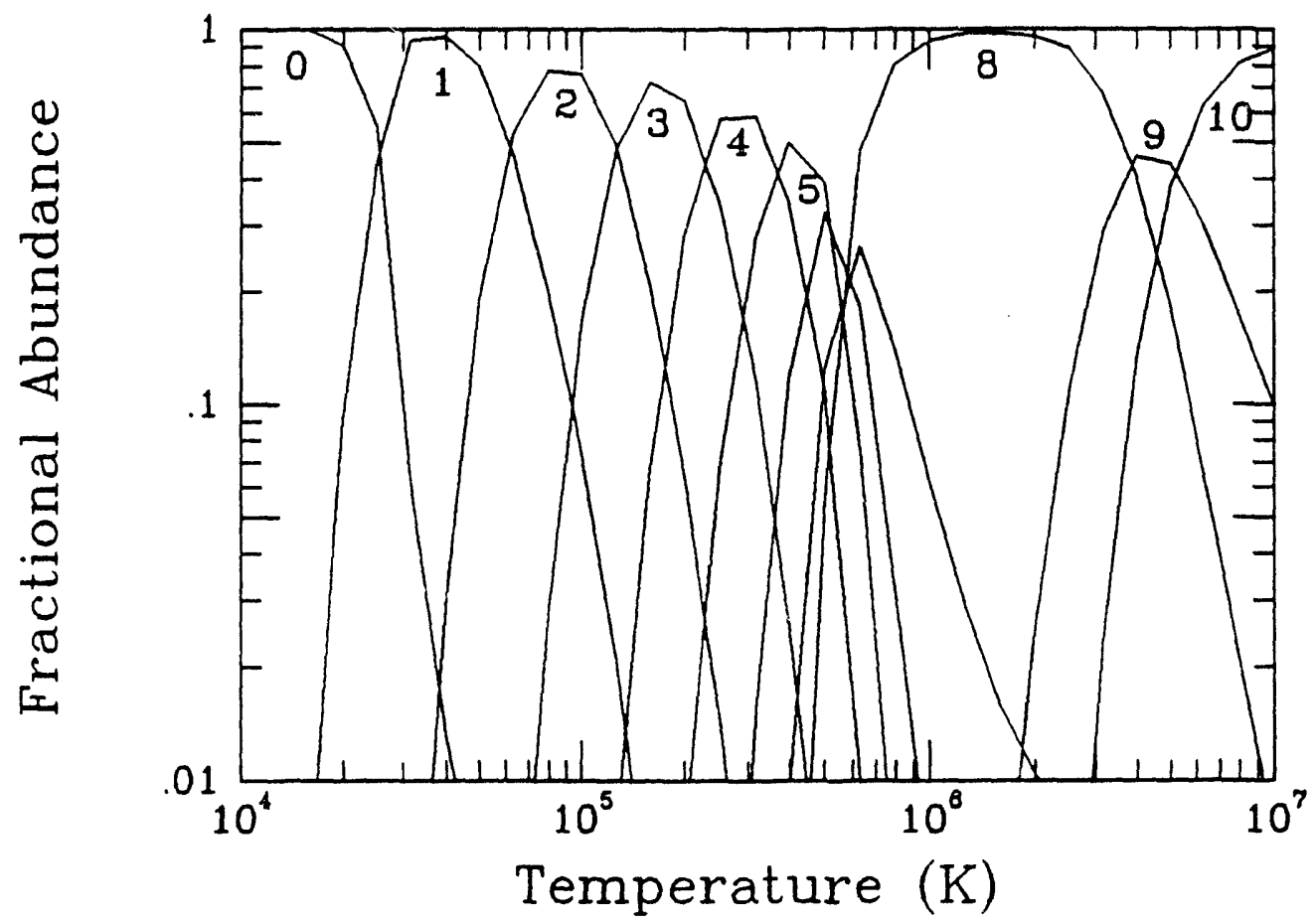

Figure 2.1: Fractional ion abundances for neon versus temperature, assuming coronal equilibrium [3]. Note how the closed-shell He-like configuration, labeled as 8 , dominates over a wide range of temperature. 
preponderance of He-like ions in most astrophysical plasmas obviousiy makes their emission important, but their line emission is also intrinsically bright because there are only two electrons. Unlike open-shell ions which may have dozens or hundreds of important transitions, He-like ions have only a few important lines since low- $n$ energy levels are generally nondegenerate and each excited level usually has only one significant decay channel. The small number of lines also tend to be close together in wavelength while still being easily resolved from each other, making quantitative spectroscopic analysis feasible. From a theoretical point of view, He-like ions are also relatively simple to model. This is important, since complete experimental examination of an atomic system is generally impossible, and it is always necessary to rely to some degree upon theory.

\subsection{The 2-to-1 Transition Lines}

A schematic energy level diagram of the He-like system is presented in Figure 2.2, showing the ground state $1 s^{2} 1 S_{0}$ level and $1 s 2 l$ excited levels. Higher $1 s n l$ levels are similar to the $1 s 2 l$ configuration with the addition of triplet and singlet $D$ terms for $n=3$, a further four $F$ terms for $n=4$, and so on. There are only four single-photon 2 to-1 transitions in He-like ions, as discussed in detail below. In contrast, there are 22 possible satellite lines from doubly excited Li-like ions, all of which lie close in wavelength to the four He-like lines. These lines, 26 in all, have been discussed in depth by Gabriel [32] and labeled with the letters of the alphabet. While most of them arise from dielectronic recombination (which will be discussed later), the four dominant lines (labeled as $w, x, y$, and $z$ ) all arise from direct collisional excitation of He-like ions. 


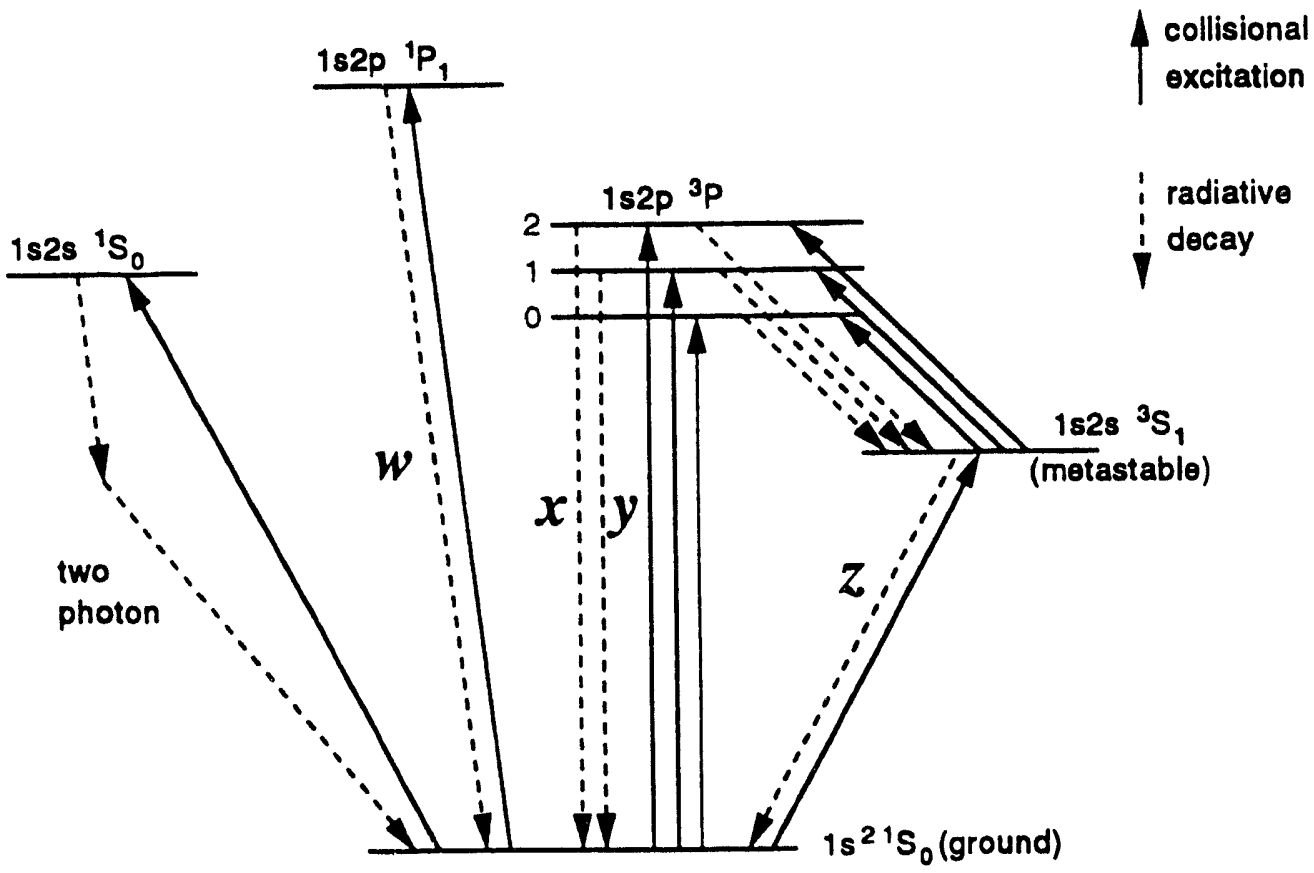

Figure 2.2: Energy level diagram showing ground state and first excited states $(1 s 2 l)$ of a He-like ion. Line $w$ is the resonance line, $x$ and $y$ are intercombination lines, and $z$ is the forbidden line. Energy differences are not to scale; all excited states are much closer to each other than to the ground state. 


\subsubsection{Direct Excitation}

The most direct and often dominant method of exciting ions is known as electron impact excitation, or EIE, in which an incoming free electron collides with an ion or atom, driving one of the bound electrons into a higher energy bound state, and reducing the energy of the free electron by an equal amount (see Figure 2.3). Obviously, for a state to be excited, the incoming electron must have an energy larger than the energy difference between the initial and final (excited) state of the ion, that is, the electron energy must be avove threshold. Since lines $w, x, y$, and $z$ are all radiative decays to ground, each has an excitation threshold equal to its photon energy.

The highest energy line, and generally the most intense, is the $1 s 2 p{ }^{1} P_{1} \rightarrow 1 s^{2}$ ${ }^{1} S_{0}$ transition producing line $w$, also known as the "resonance" line because it results from a fully allowed electric dipole (E1) radiative transition after collisional excitation from the ground state. The radiative rate $A_{r}$ for this transition is extremely high and scales as $Z^{4}$. As with most allowed transitions which are excited from ground, the excitation cross section of $w$ remains significant even at large electron energies, owing to the contribution of higher angular momenta [69]. At energies several times above threshold, the cross section falls as $\ln E / E$. The other singlet state, $1 s 2 s 1 S_{0}$, is forbidden to decay to ground via single photon emission by selection rules $(J=0$ to 0 forbidden for all multipoles) but can decay via two-photon emission (2E1), with a rate that scales as $Z^{6}$. This produces continuum emission since only the sum of the photon energies is quantized, although the probability is highest for emission of two "half energy" photons.

Although the three $1 s 2 p^{3} P$ triplet levels have the same electronic configuration and are very close in energy, with excitation rates proportional to $2 J+1(5: 3: 1$ for 


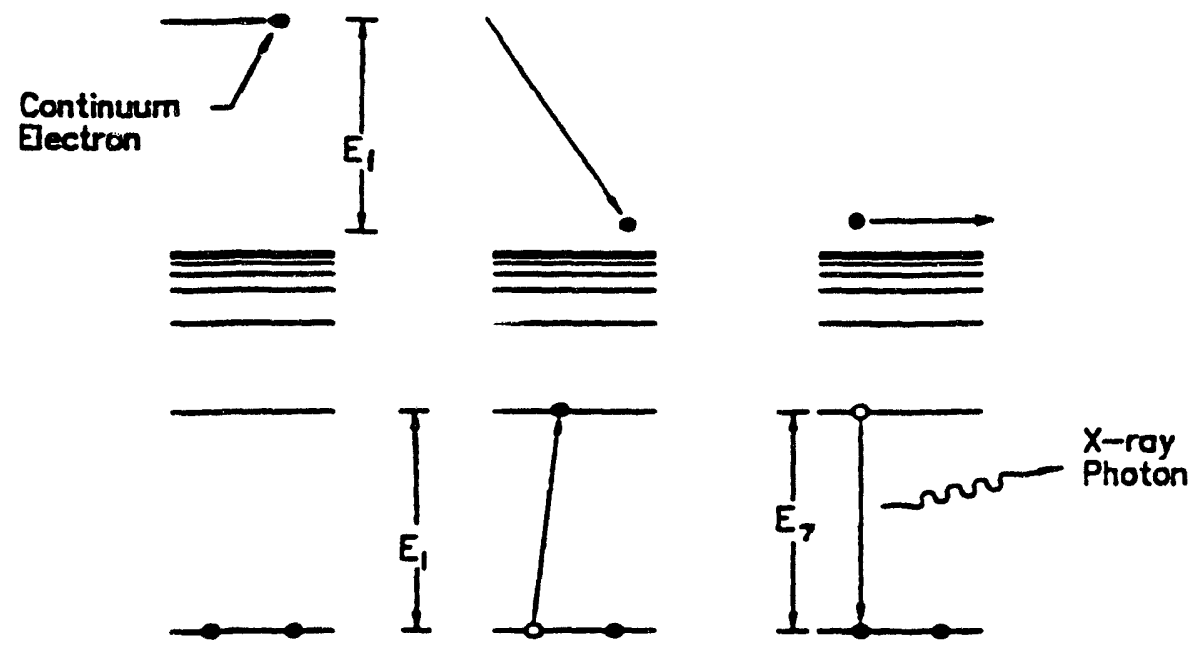

Figure 2.3: Illustration of electron impact excitation (EIE) of a He-like ion. A colliding electron transfers some of its kinetic energy to excite a bound electron into a higher energy level. 
$\left.{ }^{3} P_{2,1,0}\right)$, they decay in very different ways. The ${ }^{3} P_{0}$ level is strictly forbidden to decay to ground ( $J=0$ to 0 not allowed) and instead decays to $1 s 2 s^{3} S_{1}$. (For nuclei with nonzero magnetic moment, decays to ground can occur because of hyperfine coupling [39], but this decay mode is not available to neon ions, which all have zero nuclear spin.) The ${ }^{3} P_{1}$ level is forbidden to decay to ground by E1 (for strict LS coupling $\Delta S$ must equal 0 ), but is partially mixed with $1 s 2 p^{1} P_{1}$ because both levels have $J=1$, so that it does in fact radiate via electric dipole as line $y$, with $A_{r} \propto Z^{9}$. The ${ }^{3} P_{2}$ level can decay to ground by magnetic quadrupole ( $\Delta J=2$ with parity change) as line $x$, but the rate is very slow for low $Z$ (scaling as $Z^{8}$ ) and so it usually decays to $1 s 2 s^{3} S_{1}$. Together, lines $x$ and $y$ are known as "intercombination" lines because they require a change in spin, intercombining the triplet and singlet series. For low- $Z$ elements, $x$ and $y$ are unresolvable, and for the rest of this thesis " $x y$ " will refer to the blend of $x$ and $y$. Since collisional excitation of these triplet levels (including ${ }^{3} S_{1}$ ) requires a change in spin, only small values of angular momenta, corresponding to close encounters where spin exchange is most likely, contribute to the excitation cross section [69]. Lines $x, y$, and $z$ therefore have cross sections which decrease with energy, scaling as $E^{-a}$ with $a$ between 2 and 3 .

The lowest-lying of all $1 s 2 l$ levels is the metastable state $1 s 2 s^{3} S_{1}$ which decays via a relativistic magnetic dipole (M1) transition, producing line $z$. Early nonrelativistic calculations of the radiative rate of this level predicted it would be so slow (E1 prohibited because parity does not change, and M1 forbidden because spin does change) that 2-photon emission would dominate. After single-photon "forbidden" line emission from several ions was observed in the Sun, however [33], calculations which included the effects of relativistic momentum showed that the level decays by M1 radiation, with a radiative rate that scales as $Z^{10}$. 
As discussed previously, the ${ }^{3} S_{1}$ level producing line $z$ is fed by cascades from ${ }^{3} P_{0}$ and ${ }^{3} P_{2}$. Cascades from higher- $n$ levels can populate all six of the $1 s 2 l$ levels, but this mechanism is especially important for line $z$, which can derive more than half its intensity from cascades. As will be discussed in section 2.3, electron collisions not only populate the $3 S_{1}$ level but can, at sufficiently high densities, depopulate the metastable level via collisional transfer to the $1 s 2 p^{3} P$ levels.

\subsubsection{Innershell Ionization and Excitation}

In addition to direct excitation and cascades, line $z$ can also be excited by innershell ionization (ISI) of Li-like ions. The ground state configuration of Li-like ions is $1 s^{2} 2 s$. If one of the $1 s$ electrons is removed--the cross section for this process is of course much smaller than that for removal of the $2 s$ electron--then the resulting He-like ion will be left in either a $1 s 2 s^{1} S_{0}$ or $1 s 2 s^{3} S_{1}$ state (see Figure 2.4). The threshold for ISI of Li-like ions is slightly below that for ionization of He-like ions because of the shielding effect of the $2 s$ electron. ISI is most important when the abundance of Li-like ions is large and the electron temperature is high. While these two conditions are mutually exclusive for coronal equilibrium, such a situation can occur in ionizing plasmas such as young supernova remnants. It is also often true in tokamak plasmas, where large temperature gradients and ion transport can cause large deviations from coronal equilibrium. Unexpectedly high intensities for line $z$ were observed in several ions for many years before it was recognized that ISI was at least partially responsible [13].

A reliable indicator of the importance of ISI in production of $z$ is the strength of certain Li-like satellite lines. Although most satellites come from dielectronic recombination, a few can be collisionally excited when a $1 s$ electron, rather than being 


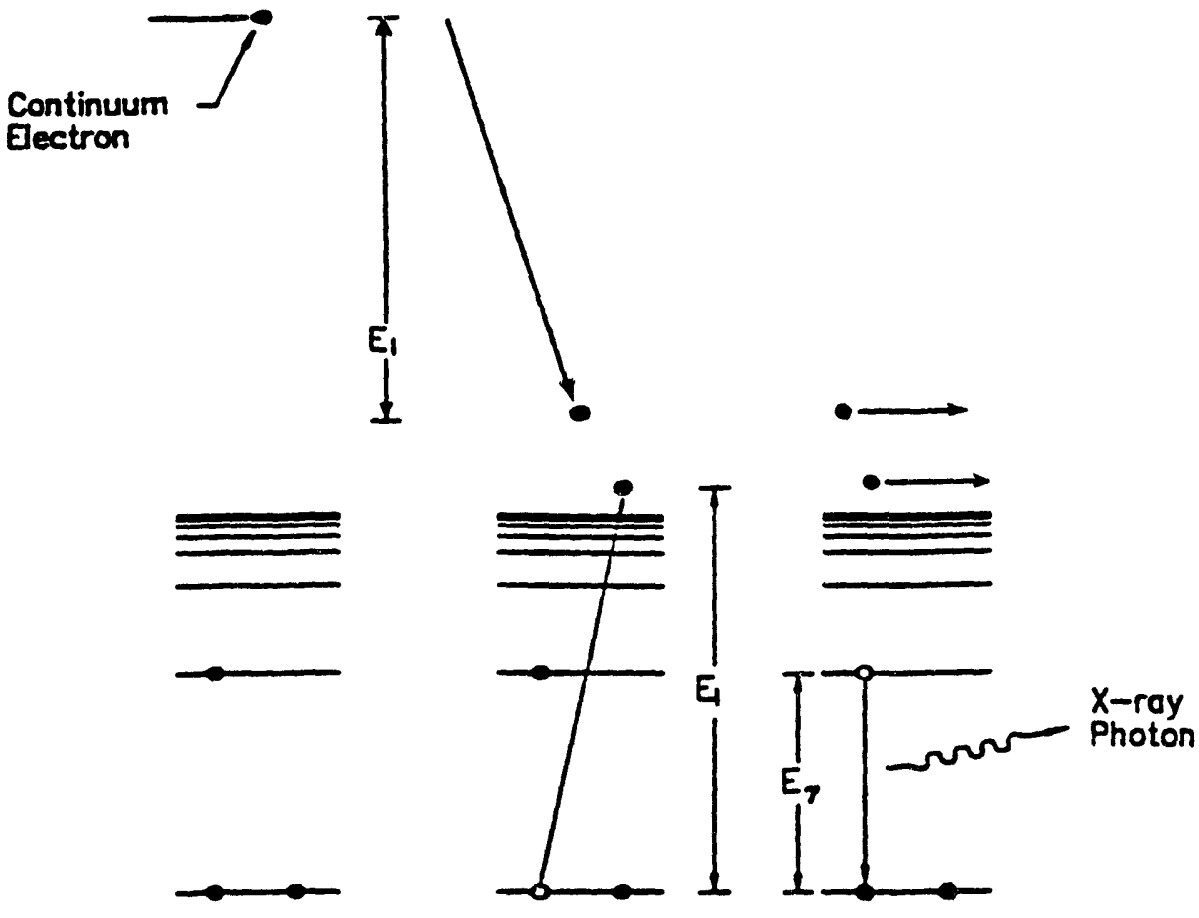

Figure 2.4: Illustration of innershell ionization (ISI) of a Li-like ion. A free electron with kinetic energy greater than the $K$-shell binding energy collides with the ion, driving one of the $1 s$ electron into the continuum while the incident electron loses an equivalent energy. The collision leaves the resulting He-like ion in either a $1 s 2 s^{3} s_{1}(75 \%)$ or $1 s 2 s^{1} S_{0}(25 \%)$ state. The decay of the ${ }^{3} S_{1}$ level produces a $z$ photon. 
completely ejected as in ISI, is instead excited to a higher level, leaving the ion in a doubly excited state. There are 16 distinct levels in the Li-like $1 s 2 l 2 l$ ' configuration, with 22 possible 2-to- 1 transitions. The extra $n=2$ "spectator" electron has little effect other than to partially shield the nuclear charge, which slightly changes the energies of the transitions. The satellite lines therefore fall within or just below (in energy) the true He-like spectrum.

Seven of the Li-like levels can be populated by collisional excitation of the ground state (to the $1 s 2 s 2 p$ configuration). These doubly excited states usually autoionize. but their radiative decays produce the lines $q$ and $r\left[1 s 2 s\left({ }^{3} S\right) 2 p 2 p_{3 / 2,1 / 2} \rightarrow\right.$ $\left.1 s^{2} 2 s^{2} S_{1 / 2}\right], s$ and $t\left[1 s 2 s\left({ }^{1} S\right) 2 p{ }^{2} P_{3 / 2,1 / 2} \rightarrow 1 s^{2} 2 s^{2} S_{1 / 2}\right]$, and $u$ and $v\left[1 s 2 s\left({ }^{3} S\right) 2 p\right.$ ${ }^{4} P_{3 / 2,1 / 2} \rightarrow 1 s^{2} 2 s^{2} S_{1 / 2}$. The "1,3 $s^{\prime \prime}$ in parentheses refers to the coupling of the $1 s$ and $2 s$ electrons. The strongest satellite is $q$, followed by $r$ and $t$. The strengths of these lines are discussed in detail in Chapter 8, but for now it will suffice to say that for Lilike $\mathrm{Ne}$, line $r$ is roughly half as strong as $q$, with which it is blended, and that all other satellites have negligible intensities when collisionally excited. A spectrum of He-like neon, including satellites, is shown in Figure 2.5.

\subsubsection{Recombination Mechanisms}

He-like $2 \rightarrow 1$ lines can also be produced when ions recombine. The three primary mechanisms for this process are radiative recombination (RR), dielectronic recombination (DR), and charge exchange recombination (CER). There are a few other recombination avenues, such as three-body (ion-electron-electron) recombination, which can be important in high density plasmas, but they are negligible in most astrophysical environments outside stellar interiors. 


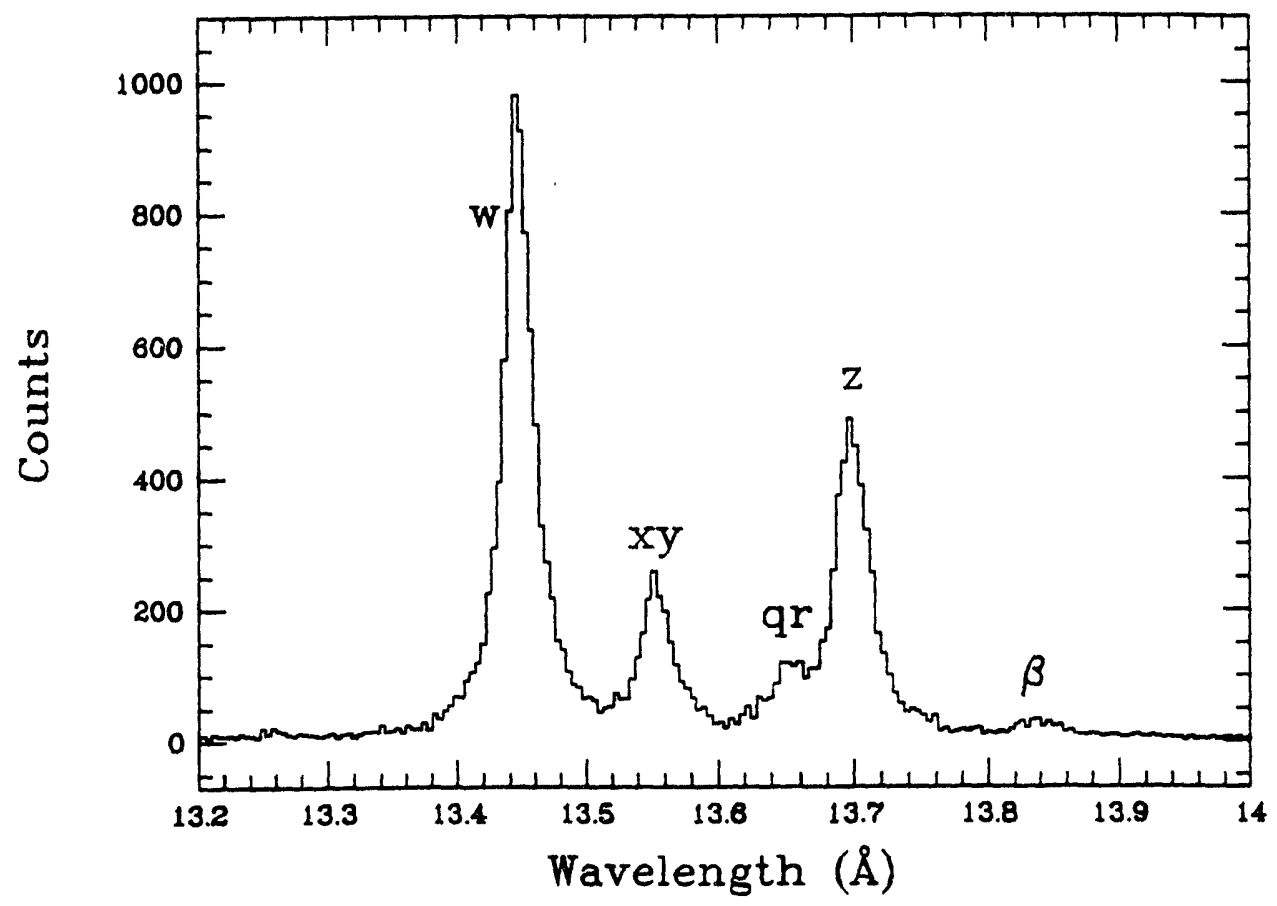

Figure 2.5: Spectrum of He-like neon obtained with EBIT at an electron energy of $1000 \mathrm{eV}$. Spectral resolving power $E / \triangle E \approx 500$, comparable to that of the upcoming $\mathrm{X}$-ray satellite missions $A X A F$ and $X M M$. Lines $q r$ and $\beta$ are, respectively, Li-like and Be-like collisional satellites of $w$. 
Radiative recombination is simply the inverse of photoionization, and is a twobody process in which a free electron collides with an ion and is captured into a bound state of the ion. A photon is emitted with an energy equal to the electron's initial kinetic energy plus the binding energy of the level into which it is captured. This process is shown schematically in Figure $2.6 a$ and can be written as

RR: $\quad A^{q+}+e^{-} \rightarrow A^{(q-1)+}+\gamma$.

The recombining electron is most likely to be captured into a low energy state, but often ends up in a high- $n$ level from which it will then cascade directly or indirectly to ground. He-like $2 \rightarrow 1$ transitions can thus be excited by radiative recombination of $\mathrm{H}$ like ions. The He-like triplet lines have larger effective cross sections for excitation by $R R$ than the resonance line $w$ because of their higher statistical weights $(2 J+1)$ and the effect of cascades. Therefore anomalously high intensities of $x, y$, and $z$ relative to $w$ are a signature of recombination-dominated plasmas (such as cooling or photoionized plasmas).

Dielectronic capture is like RR except that instead of carrying away the recomuination energy via a photon, one of the already bound electrons is excited to a higher-energy level. Since bound-state energy levels are quantized, this process is resonant, i.e., it can only occur at discrete energies. After dielectronic capture the ion is left in a doubly excited state (see Figure $2.6 b$ ) which will decay either radiatively (resulting in dielectronic recombination) or by Auger emission (resulting in resonant excitation or resonant scattering). Note that this is the same situation that occurs as a result of innershell impact excitation; the only difference is which doubly excited states tend to be produced.

Auger decay, also known as autoionization, is the exact inverse of dielectronic capture; one of the excited electrons falls to a lower energy level while the other 
(a)
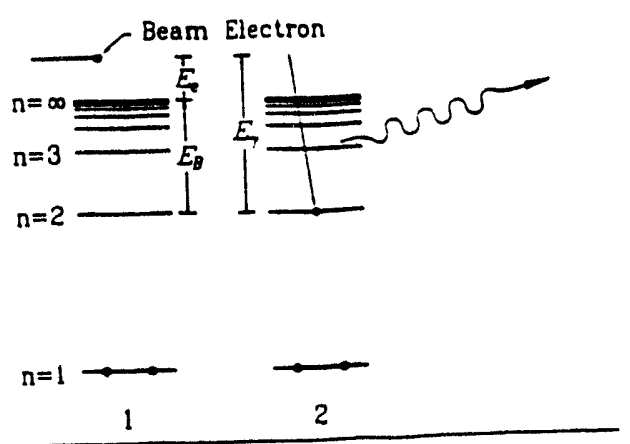

(b)

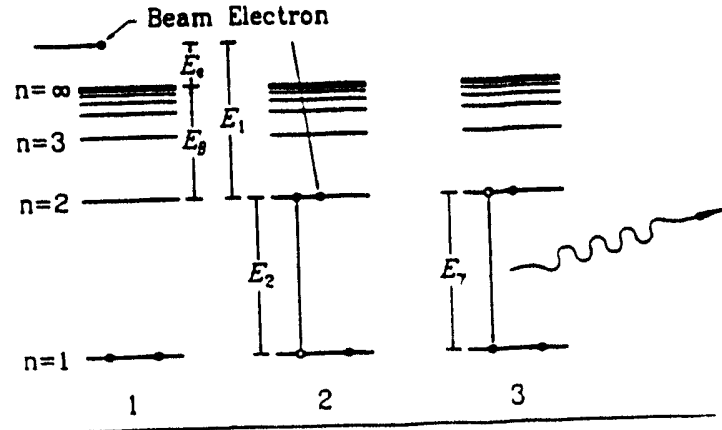

(c)
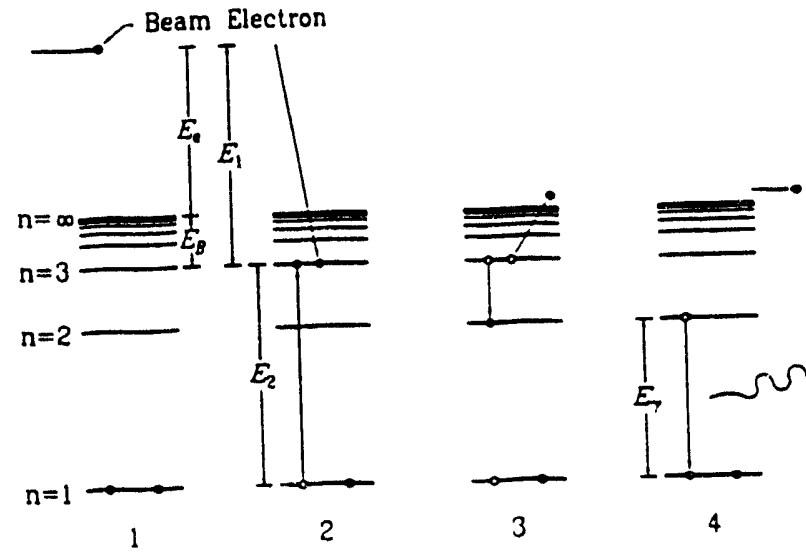

Figure 2.6: Recombination mechanisms. (a) Radiative recombination of He-like ion consists of capture of a free electron (in this case into $n=2$ level) accompanied by photon emission. Photon energy equals the sum of initial electron energy and binding energy of the final state. (b) Dielectronic recombination, for the $K L L$ resonance. When $E_{1}=E_{2}$, a free electron may be captured into a bound state by exciting an already bound electron into a higher energy level. This is known as dielectronic capture (steps 1 and 2). Dielectronic recombination occurs if the doubly excited state then decays radiatively, producing a satellite line (step 3). (c) Resonant excitation (shown here for the $K M M$ resonance) occurs if the doubly excited state instead autoionizes, leaving the ion with the same number of electrons with which it started but in a singly excited state (step 3) which then decays (step 4). If, in step 3, the doubly excited state autoionizes back to ground, leaving the ion in its original unexcited state, the process is called resonant elastic scattering (not shown). Figure taken from Ref. [49]. 
excited electron carries away the energy of that transition by being ejected into the continuum. Resonant elastic scattering (RS) is defined as dielectronic capture followed by an Auger decay which leaves the ion in its initial energy state and can be written as

RS: $\quad A^{q+}+e^{-} \rightarrow\left\{A^{(q-1)+}\right\}^{* *} \rightarrow A^{q+}+e^{-}$.

If the Auger decay proceeds to a bound state different from the original state, the resulting singly excited state will decay radiatively (see Figure $2.6 \mathrm{c}$ ) in the process known as resonant excitation (RE):

RE: $\quad A^{q+}+e^{-} \rightarrow\left\{A^{(q-1)+}\right\}^{* *} \rightarrow\left\{A^{q+}\right\}^{*}+e^{-} \rightarrow A^{q+}+e^{-}+\gamma$.

$\mathrm{RE}$ leaves the ion in its original charge state and occurs at electron energies above the direct excitation threshold of the emitted line.

If the doubly excited state (following dielectronic capture) decays radiatively to a bound singly excited state the process is known as dielectronic recombination (DR) and the ion gains one electron (see Figure 2.6b):

DR:

$$
A^{q+}+e^{-} \rightarrow\left\{A^{(q-1)+}\right\}^{* *} \rightarrow\left\{A^{(q-1)+}\right\}^{*}+\gamma .
$$

DR occurs at electron energies below the direct excitation threshold and results in the emission of a satellite line. After the satellite line is emitted, the ion is still in a (singly) excited state and emits another photon. As an example, when a He-like ion undergoes DR, the first photon (from the doubly excited Li-like ion) is a satellite of a He-like line, while the second photon (from a singly excited state) is a low-energy $\mathrm{Li}$ like line. When a H-like ion undergoes DR, however, the first photon is a :-like satellite while the second photon (from a singly excited He-like state) is a true He-like line. 
DR and RE resonances are usually labeled using the notation for the inverse Auger process, e.g., $K L M$ denotes the DR resonance in which a $K$-shell electron is excited to the $L$ shell by the capture of a continuum electron into the $M$ shell. Likewise, a $2 \rightarrow 1$ photon can be produced from a $K M M$ RE resonance when a $K$-shell electron is excited to the $M$ shell by capture of a free electron into the $M$ shell, followed by the Auger decay of one of the $M$-shell electrons to the $L$ shell, ejecting the other $M$-shell electron and leaving an $L$-shell electron which then decays to ground. DR and RE can sutstantially increase the excitation cross section of an emission line (and its satellites), particularly for higher- $Z$ elements.

Charge exchange recombination (CER) occurs when an atom or ion collides with another ion, resulting in the transfer of an electron. CER usually occurs between an ion and a neutral atom because ions repel each other. This is a resonant process, as the ionization energy of the atom (before transfer) must equal the ionization energy of the ion (after transfer). A striking example of this process is seen in tokamaks, where neutral hydrogen atoms $(\mathrm{IP}=13.6 \mathrm{eV})$ can donate electrons to bare $\mathrm{Na}$ nuclei, with the electron being transferred into a $7 p$ orbital, resulting in strongly enhanced $\mathrm{Na}$ Lyman- $\zeta$ emission. CER is not as important in astrophysical $\mathrm{X}$-ray sources, since there are generally no neutral atoms present. One exception, however, is the case where a cold cloud of neutral gas is being evaporated by a hot $\mathrm{X}$-ray plasma.

\subsection{Density Diagnostics}

The use of He-like line ratios as diagnostics of plasma conditions, particularly density and temperature, was first described by A. H. Gabriel and C. Jordan in 1969 [34], soon after their observation of strong forbidden line emission from several Helike ion species in the Sun [33]. The atomic term diagram in Figure 2.2 helps to 
illustrate the physical origin of the density sensitivity of the He-like lines. At low densities, any excitation of an ion is immediately followed by spontaneous radiative decay (or perhaps Auger decay if the ion is doubly excited) before any further collision can occur. Since the $1 s 2 p^{3} P$ and $1 s 2 s^{3} S_{1}$ levels are so close in energy, their relative populations are only weakly affected by temperature, and the intensity ratio of the intercombination ( $x$ and $y$ ) and forbidden $(z)$ lines is approximately constant over a wide range of temperature.

With increasing electron density, however, the rate of collisional excitation from the ${ }^{3} S_{1}$ level to the ${ }^{3} P$ levels becomes comparable to the radiative decay rate of the metastable ${ }^{3} S_{1}$ level, and lines $x$ and $y$ grow stronger at the expense of $z$ (see Figure 2.7). Because of this collisional transfer, a fairly small change in electron density can dramatically alter the intensities of $x$ and $y$ relative to $z$. Observation of these lines in cosmic X-ray sources can therefore provide an excellent means of accurately determining the local electron density. As seen in Figure 2.8. the density at which the line ratio $R$, defined as $I_{z} /\left(I_{X}+I_{y}\right)$, is most sensitive to density changes depends upon the particular elemental species. This is mostly because the radiative rate of line $z$ scales as $Z^{10}$. This diagnostic technique may therefore be applied over a wide range of densities using He-like ions of abundant elements such as $\mathrm{C}, \mathrm{N}, \mathrm{O}, \mathrm{Ne}, \mathrm{Mg}, \mathrm{Al}, \mathrm{Si}$. $\mathrm{S}, \mathrm{Ar}, \mathrm{Ca}$, and $\mathrm{Fe}$. The usable range for this diagnostic technique extends from less than $10^{8} \mathrm{~cm}^{-3}$ for carbon to over $10^{17} \mathrm{~cm}^{-3}$ for iron [71]. As shown by Gabriel and Jordan, the equation describing $R$ versus electron density may be written as

$$
n_{e}=n_{c}\left(\frac{R_{0}}{R}-1\right)
$$

where $R_{O}$ is the low density limit of $R$, and $n_{C}$ is the "critical density" at which $R$ falls to one half of $R_{0}$. 


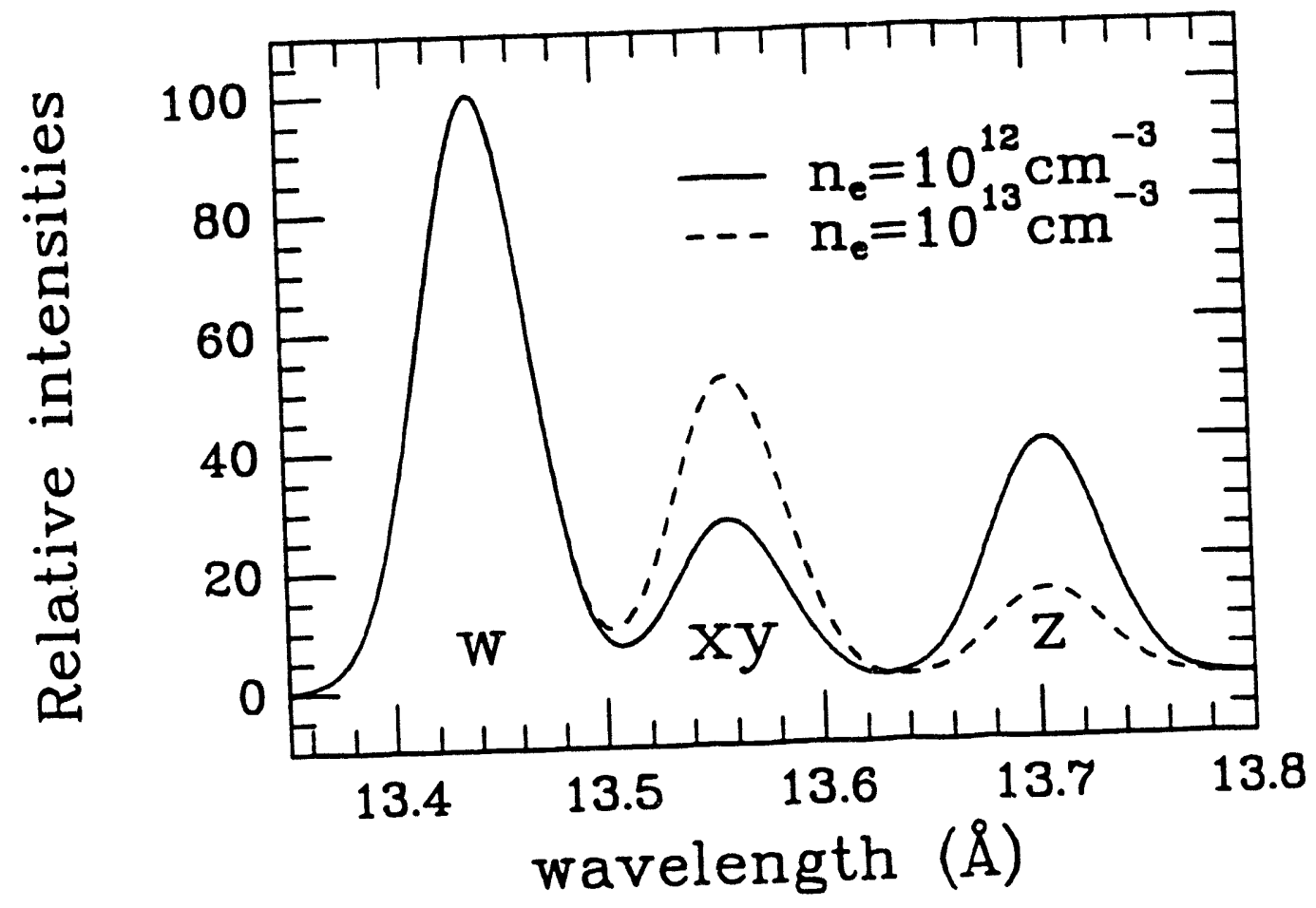

Figure 2.7: Predicted line intensities for $\mathrm{He}$-like $\mathrm{Ne}^{8+}$, with electron energy $1000 \mathrm{eV}$. Note how the relative intensities of $x y$ and $z$ differ dramatically for the two electron densities. 


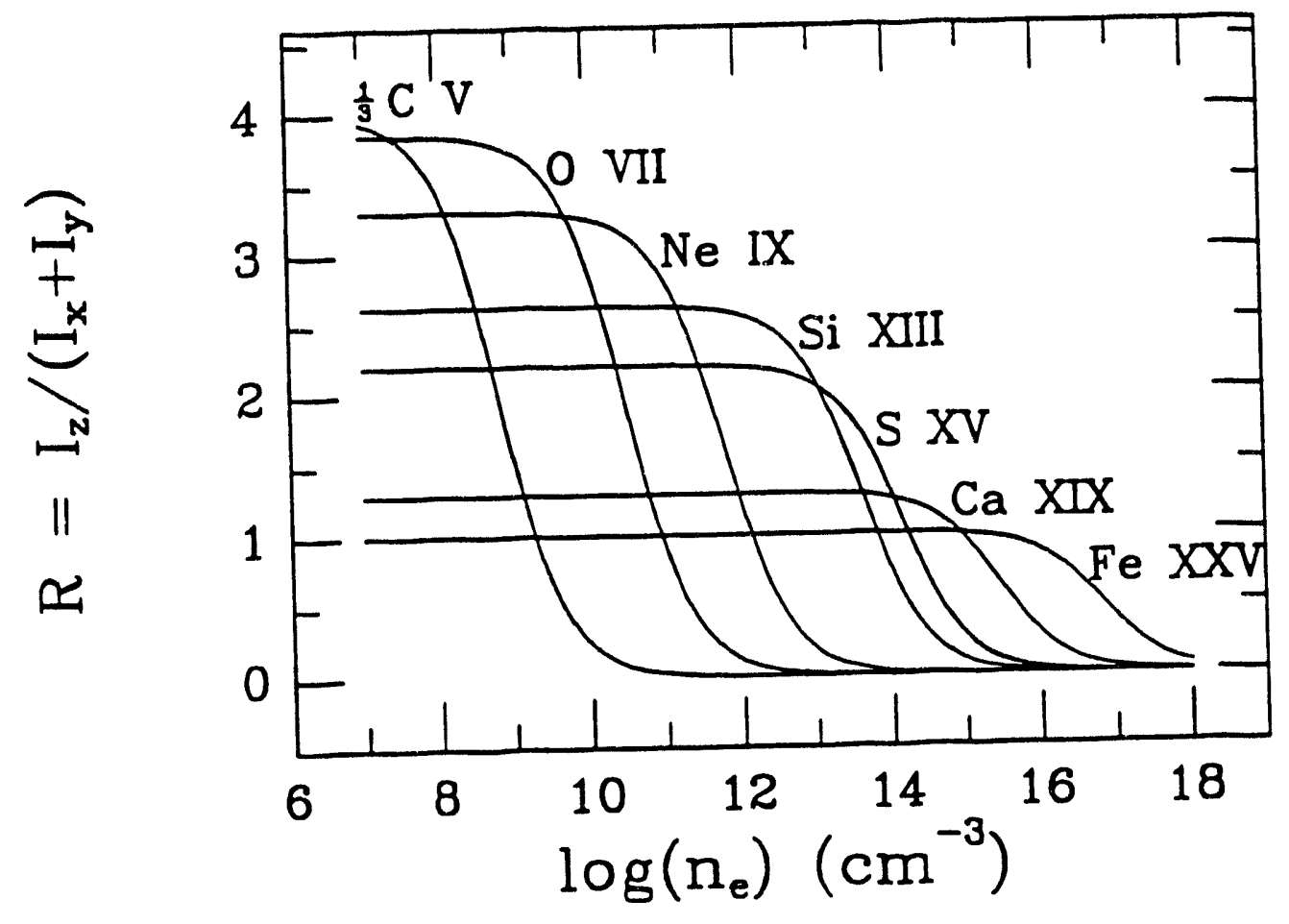

Figure 2.8: The line ratio $R=I_{z} /\left(I_{x}+I_{y}\right)$ for several abundant He-like ions, computed from theoretical results of Pradhan [68]. Note how each ion species is sensitive at a different electron density. For carbon, $R$ is shown divided by 3 . 


\subsection{Temperature Diagnostics}

Helium-like ions can also serve as temperature diagnostics in X-ray sources. This is due to the very different dependences on energy of the collisional excitation rates to the various levels. As described previously, the collisional excitation cross section for $w$ increases with electron energy due to the contribution from higher angular momentum states, eventually decreasing as $\ln E / E$ (see Figure 2.9). The EIE cross sections for $x, y$, and $z$, however, are dominated by terms associated with electron exchange which peak at low values of the electron angular momentum, and thus decrease with increasing energy according to a fairly steep power law. The line ratio $G=\left(I_{x}+I_{y}+I_{z}\right) / I_{w}$ is therefore very sensitive to temperature. At the same time, $G$ is essentially independent of electron density, since electron-ion collisions simply transfer electrons between the ${ }^{3} S_{1}$ and ${ }^{3} P$ states. (A small fraction of collisions excite higher- $n$ levels, but nearly all of those states then decay back to $2^{3} P$ and $2^{3} S_{1}$.) The utility of He-like ions as temperature probes is clearly illustrated in Figure 2.10, which shows some theoretical results of Pradhan [68]. By observing the value of $G$ for a given He-like ion and then comparing with a reference curve, the local temperature of an emitting region in coronal equilibrium can be determined. This diagnostic technique is applicable over a temperature range of a few times $10^{5}$ to over $10^{8} \mathrm{~K}$. which spans virtually the entire range of temperatures intrinsic to cosmic X-ray sources.

\subsection{Ionization Balance Diagnostics}

Some care must be exercised when applying the diagnostics discussed above because up to three ion species contribute to each line's intensity. Besides direct 

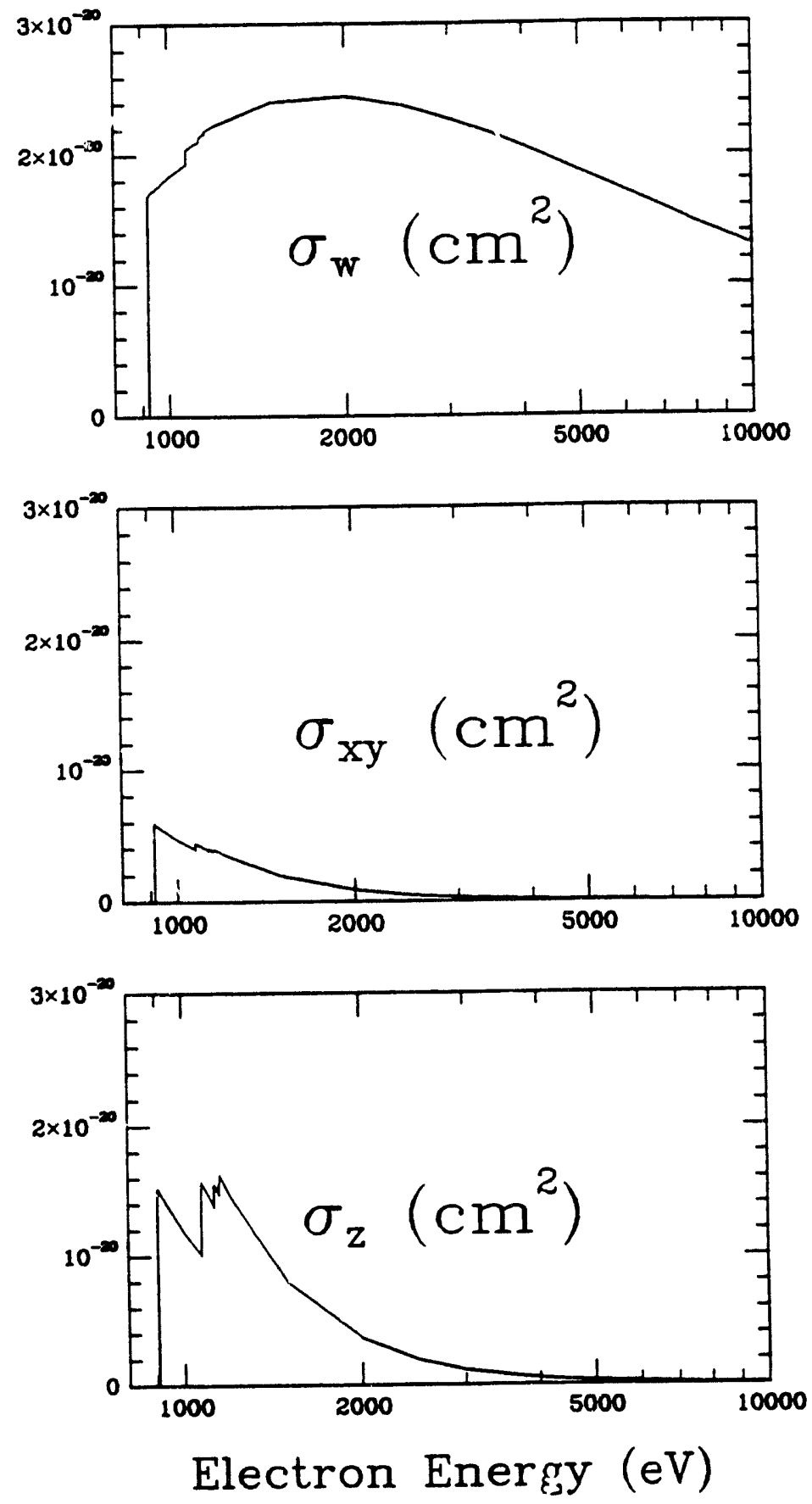

Figure 2.9: Electron impact excitation cross sections (after including cascades) for the $K_{\alpha}$ lines of He-like neon, based upon calculations by Reed [72]. The energy dependence of lines $x y$ and $z$ is very different from that of $w$, making the intensity ratio $G=\left(I_{x y}+I_{z}\right) / I_{w}$ sensitive to temperature. 


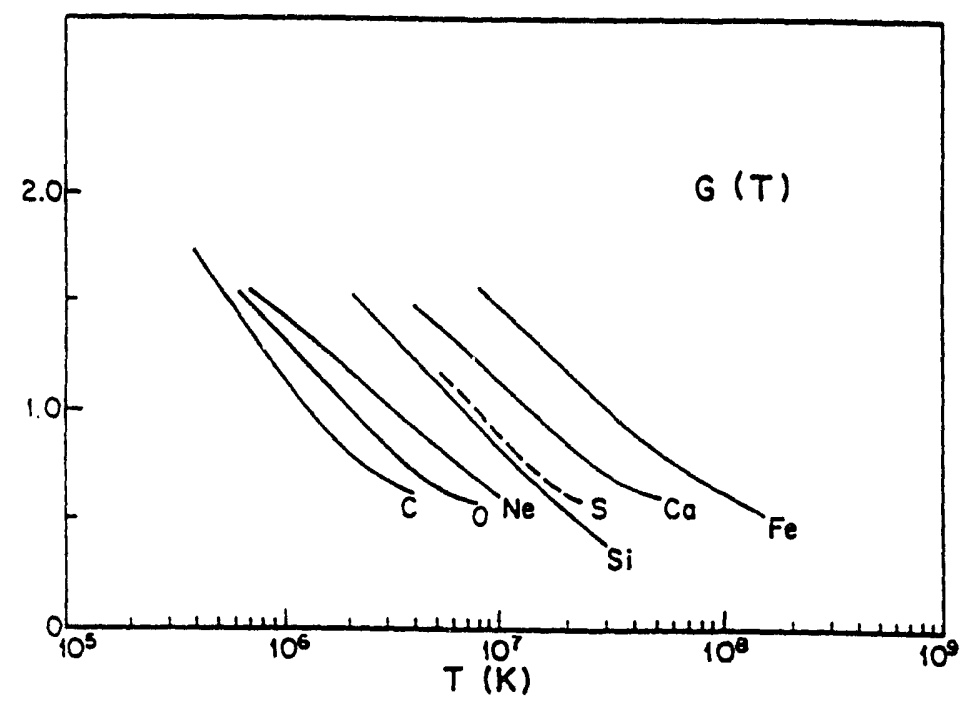

Figure 2.10: The line ratio $G=\left(I_{x}+I_{y}+I_{z}\right) / I_{w}$ as a function of temperature, as computed by Pradhan [68] assuming coronal equilibrium. $G$ is sensitive to temperature over a different range for each element. 
excitation and dielectronic recombination of $\mathrm{He}$-like ions, there is recombination of $\mathrm{H}$ like to He-like ions which enhances $x, y$, and $z$ more than $w$, and innershell ionization of Li-like ions which increases the intensity of $z$ (and therefore $x$ and $y$ if the density is high enough). Naively assuming coronal equilibrium, where the ratio of $\mathrm{H}-, \mathrm{He}-$, and Li-like ions is determined strictly by temperature, can lead to wildly inaccurate temperature estimates if the plasma is recombining, ionizing, or nonthermal.

The contribution of ISI can be determined by the strength of the Li-like satellite $q$ (or $r$ if sufficiently intense). It is collisionally excited just like $w$, but with a $2 s$ spectator electron that slightly increases the wavelength; like $w$, it even has its own DR satellites. Its EIE cross section is proportional to that of $w$, so that the ratio of $\mathrm{Li}$ like to He-like ions can be easily determined. If the cross section for ISI is known, the fraction of $z$ that comes from ISI can then be directly determined from the strength of q. Likewise, the relative abundances of Li-like and He-like ions can also be used to deduce the temperature by comparison with theoretical models of ionization balance. If the temperatures derived from $I_{q} I_{w}$ and $G$ are not consistent, then the plasma is not in ionization equilibrium.

The contribution of recombining $\mathrm{H}$-like ions can in principle be inferred from the intensity of the relatively nearby Lyman- $\alpha$ line $\left(\lambda_{w} / \lambda_{\mathrm{Ly}-\alpha}=1.1\right)$, but in practice this is somewhat more complicated because Lyman- $\alpha$ can itself be produced by the recombination of bare nuclei. Because, however, recombination cross sections are generally two or three orders of magnitude smaller than those for excitation and ionization, recombination generally will not be an important source of He-like line excitation except in photoionized plasmas. The anomalously large $G$ ratio and negligible intensity of $q$ in such a case would make it obvious that the plasma was recombination-dominated. 


\subsection{Existing Data, Calculations, and Applications}

Since the original work of Gabriel and Jordan, several groups $[1,15,60,62,63,71]$ have worked on more detailed theoretical models in an effort to produce more reliable values of $R$ and $G$ as functions of electron density and temperature. As we have seen, however, many processes are involved in He-like $\mathrm{K}_{\alpha}$ emission. One must take into account collisional excitation, innershell ionization and excitation, radiative recombination, dielectronic recombination, resonant excitation, autoionization, radiative cascades, and collisional transfer. Because of quantitative uncertainties in all these atomic and radiative processes, published results of the several authors listed above sometimes vary significantly. When presented with the same observational data, the different models may, for example, predict electron densities that vary by a factor of ten or more [71]. Published works also almost invariably assume coronal equilibrium, rather than present results in a more flexible manner that can be employed over a wide range of conditions. This makes it difficult to compare one model against another or with data obtained under nonequilbrium conditions.

Clearly, experimental data are required to determine which theoretical calculations are correct. Some tests have come from experimental observations of the solar corona ( $\mathrm{O}$ and $\mathrm{Ne}$ [59]; $\mathrm{Ne}$ [84]; $\mathrm{Ca}[46]$ ) and tokamak plasmas ( $\mathrm{S}, \mathrm{Cl}, \mathrm{Ar}$ [47]; Fe [14]; $\mathrm{C}$ and $\mathrm{O}$ [78]; Si [48]). These have sometimes pointed to significant deficiencies in the models.

None of the above observations, however, can supply what is really needed: precise measurements of cross sections and rates for collisional excitation, dielectronic recombination, resonant excitation, radiative recombination, and innershell ionization under controlled conditions, and as functions of energy. Until a few years ago such experiments would hardly have been conceivable, but a new 
device called an electron beam ion trap has made many of these measurements relatively straightforward. This device is described in the next chapter. 


\section{Chapter 3}

\section{The Electron Beam Ion Trap}

In plasmas, such as found in tokamaks and discharge tubes, $\mathrm{X}$-ray emission is the result of the interaction of many different processes excited by a continuous distribution of particle energies. Very high-quality X-ray spectra may be obtained, and plasma parameters such as temperature and density can be determined from Thompson scattering measurements (for example), but it is generally impossible to determine the cross sections of individual atomic processes. In a crossed-beam apparatus, by contrast, intersecting beams of ions and electrons have a single interaction energy, and absolute ionization cross sections can be determined very accurately by measuring the relative numbers of different ion species before and after interacting with the electron beam. Crossed-beam experiments, however, are generally limited to the study of ions with relatively low charge, and observations of $\mathrm{X}$-ray emission are difficult because of the small ion-electron interaction rates.

Ion accelerators can easily produce highly charged ions, and X-ray line emission can be generated by directing the ion beam through a target, often a thin carbon foil. Like crossed-beam experiments, interactions between the ions and electrons (bound within the atoms of the foil) take place at a single energy, but the energy is typically several hundred $\mathrm{MeV}$, far above the thresholds of all atomic processes. Additional complications arise because the electron density in the interaction region (the solid foil) is extremely high, of order $10^{23} \mathrm{~cm}^{-3}$, creating a significant population of excited metastable states. It is also difficult to obtain high-resolution X-ray spectra because the high velocity of the emitting ions introduces substantial Doppler shifts which vary with observation angle. 
In an Electron Beam Ion Trap (EBIT), the device used for this investigation of He-like neon, stationary ions are probed with a tunable, nearly monoenergetic electron beam (typically $\leq 50 \mathrm{eV} \mathrm{FWHM),} \mathrm{and} \mathrm{high-resolution} \mathrm{spectra} \mathrm{are} \mathrm{accumulated} \mathrm{as} \mathrm{a}$ function of electron energy. An EBIT also affords a great deal of control over which charge states are present, and ions can be studied while ionizing, recombining, or in ionization equilibrium. The combination of high-resolution $\mathrm{X}$-ray spectroscopy and detailed control over ion-electron interaction parameters permits the determination of cross sections for individual excitation processes such as collisional excitation, innershell ionization, resonance excitation, and dielectronic recombination.

Since its completion in 1987, the EBIT at the Lawrence Livermore National Laboratory has been used with great success to study the physics of highly charged ions, making many first-of-their-kind measurements. EBIT's high resolution X-ray spectroscopy capabilities have been used to determine line overlaps for resonant photo-pumping of X-ray lasing transitions [8], measure relativistic and QED wavelength shifts in very highly charged ions such as Ne-like $\mathrm{Yb}^{60+}[12]$, and identify highly forbidden transitions such as magnetic octupole decay in $\mathrm{Ni}$-like $\mathrm{Th}^{62+}$ and $\mathrm{U}^{64+}$ [10]. Cross section measurements are a staple of the EBIT program, with studies of impact excitation of $\mathrm{He}-\mathrm{like} \mathrm{Ti}^{20+}, \mathrm{V}^{21+}, \mathrm{Cr}^{22+}, \mathrm{Mn}^{23+}$, and $\mathrm{Fe}^{24+}$ [9], dielectronic recombination in He-like $\mathrm{Ni}^{26+}$ [50] and $\mathrm{Ne}-$ like $\mathrm{Au}^{69+}$ [75], and ionization of Li-like $\mathrm{Cr}^{21+}\left(K\right.$-shell) [4.8] and $\mathrm{Ba}^{53+}(L$-shell) [85]. There have also been measurements of line polarization in $\mathrm{He}$-like $\mathrm{Sc}^{19+}[39]$ and the determination of the radiative rate of the forbidden line $z$ in He-like $\mathrm{Ne}^{8+}$ [83], which is described in Chapter 7 .

There are actually two EBITs currently in operation. Many of the experiments mentioned above were conducted on EBIT2, a slightly enhanced version of EBIT1 that was completed in 1990. After EBIT2 was fully operational, EBIT1 was upgraded to SuperEBIT, capable of reaching electron energies of almost $200 \mathrm{keV}$, high enough 
to completely ionize uranium. The experiments that are the focus of this thesis were performed on EBIT2, which will now be described in more detail.

\subsection{Major Components}

The Livermore EBIT is a modified Electron Beam Ion Source, or EBIS, explicitly built to study $\mathrm{X}$-rays emitted from the interaction of highly charged ions with an electron beam. It is described in detail by Levine et al. [52]. Magnetic fields confine and focus the electrons, which can be accelerated to any energy between a few hundred and 30,000 electron volts. Neutral atoms or ions with low charge are injected into the nearly monoenergetic beam where they are collisionally ionized and excited. As the electrons pass through a short "trap" region, the beam is compressed by a strong magnetic field. There the ions are longitudinally confined within a potential well where they may be observed for an extended period before the next ion injection occurs. Six observation ports ring the trap region, permitting several spectrometers and detectors to be used simultaneously.

The electron beam originates from a Pierce-type electron gun which is positioned at the bottom of EBIT (Figure 3.1). The cathode is made of tungsten, approximately $3 \mathrm{~mm}$ in diameter with a spherically concave emitting surface, impregnated with barium which has a very low work function. Typical operating temperatures are around $1000^{\circ} \mathrm{C}$. The adjustable anode voltage controls the current, which can be up to $200 \mathrm{~mA}$, while a focus electrode is used to make the emitted electrons converge to a single point. After passing through the hollow anode, the electron beam is guided upward toward the trap region by a pair of "transition" and "snout" electrodes. 


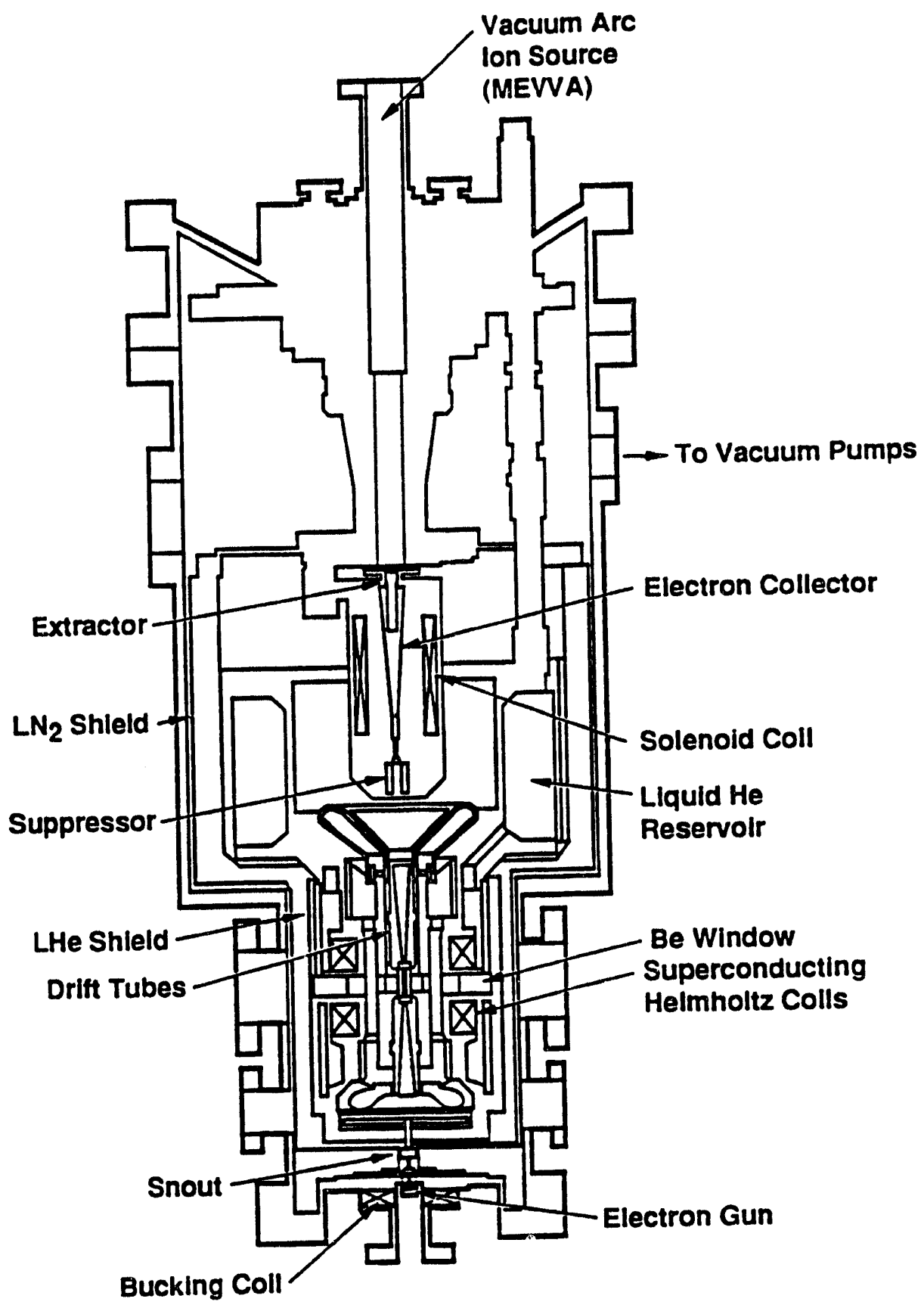

Figure 3.1: Components of the Electron Beam Ion Trap. 
A pair of superconducting Helmholtz coils create a 3-Tesla magnetic field which compresses the electron beam to a diameter of approximately $70 \mu \mathrm{m}$ as it passes through the trap region where the ions are held. The niobium-titanium coils carry 150 amperes of current and are cooled by liquid helium. Two pairs of steering coils located on the exterior of the vacuum vessel are used to fine tune the magnetic field direction so it exactly coincides with the electron beam axis. A "bucking coil" surrounding the electron gun cancels the field created by the superconducting coils so that electrons emitted from the cathode are "born" in a zero-field environment. This lets the electrons be focused more tightly than if they had to spiral around residual magnetic field lines.

The electron beam density, and therefore the ion-electron interaction rate, is highest in the trap region (typically a few times $10^{12} \mathrm{~cm}^{-3}$ ) which consists of three drift tubes (see Figure 3.2). The middle drift tube, which has six slots to allow observation of emitted X-rays, is $2 \mathrm{~cm}$ long and is typically biased about 200 volts below the two end drift tubes to create a potential well that axially confines the ions. Radial confinement is provided by electrostatic attraction of the electron beam. All three drift tube voltages float on top of a potential that can reach $30 \mathrm{kV}$, supplied by a low-noise high-voltage amplifier.

After passing through the trap, the electron beam is decelerated and dumped on the walls of the collector, which has its own electromagnet that makes the beam diverge before impact. Both the collector and its magnet are cooled by liquid nitrogen. A "suppressor" electrode prevents electrons that scatter off the collector from escaping back toward the trap. The entire path traveled by the electrons is only $40 \mathrm{~cm}$, and the beam is maximally compressed for only $2 \mathrm{~cm}$ as it passes through the trap. This short length makes the beam much more stable than that in an EBIS, which suffers from a variety of beam instabilities such as the modified rotational two-stream 

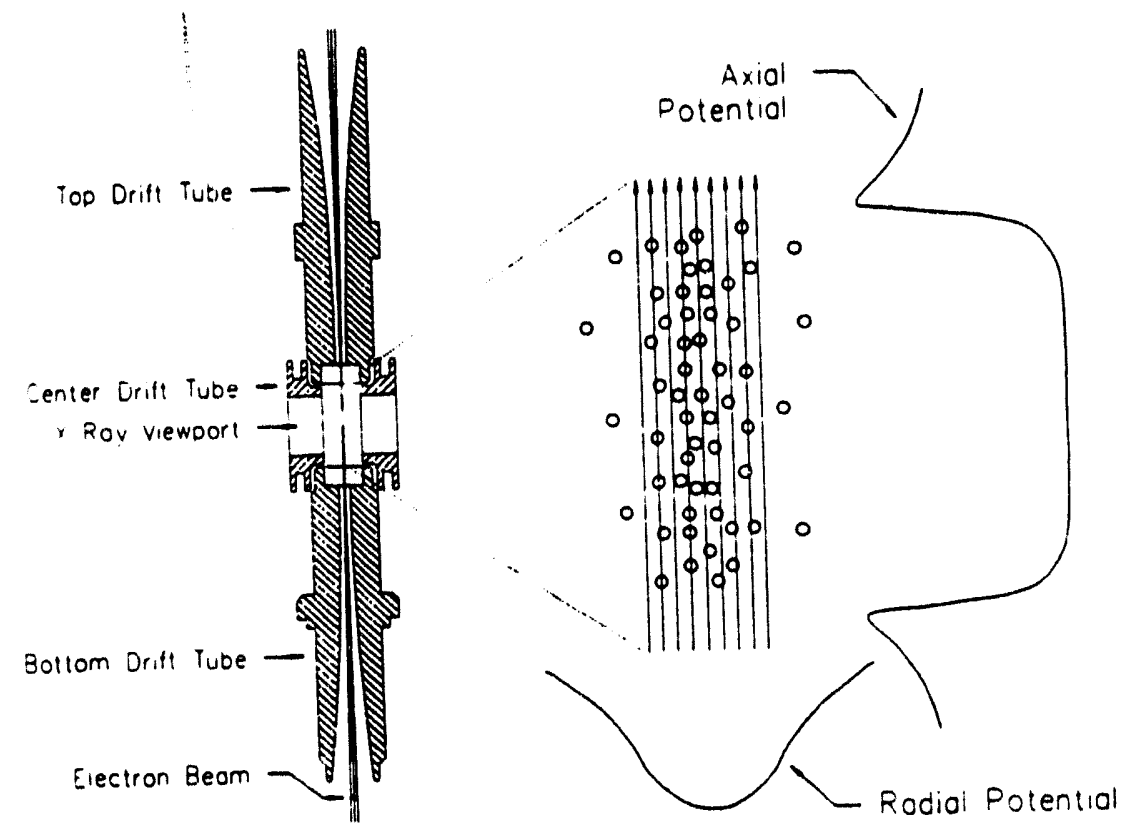

Figure 3.2: Drift tube assembly, showing the top, middle, and bottom drift tubes which are separated by thermally conducting sapphire insulators. The axial potential well is generated by raising the voltage of the two end drift tubes above that of the middle. Ions are radially confined by the electrostatic attraction of the electron beam, which also collisionally ionizes and excites the ions. From Ref. [49]. 
instability [53]. Greater stability means a lower rate of ion heating, so that ions can undergo more ionizing collisions before they diffuse out of the beam. EBIT is thus able to reach much higher charge states than a conventional EBIS, and the higher electron density also increases ion-electron interaction rates.

Ions can be introduced into the trap in two ways. The most commonly used source of ions is the metal vapor vacuum arc (MEVVA) which generates a $20-\mu$ s pulse of mostly doubly and triply charged ions on the top end of EBIT, above the electron collector. Virtually all metallic elements can be used, and some success has been achieved with nonmetals by using packed powders or conductive composite materials. The MEVVA anode and an "extractor" electrode help direct ions down the EBIT axis through a hole in the center of the electron collector, but only a small fraction of the ions produced in each MEVVA firing reach the trap. To capture ions in the trap, the top drift tube voltage must be lowered and then raised just after the burst of ions arrives, acting as a sort of drawbridge.

The other method for introducing target ions, and the one used in this study of neon, is to inject gases directly into the trap through one of the six observation ports ringing the trap. A differential pumping scheme directs a finely controlled and collimated stream of neutral atoms or molecules into the electron beam (see Figure 3.3). Neutral atoms are not affected by the electric and magnetic fields in the trap, but once an atom is ionized, it becomes trapped. Neutral atoms can also be introduced into EBIT by sublimation of a liquid or solid placed under vacuum near one of the observation ports. Although somewhat difficult to control, this method has been used successfully with sulfur.

In addition to being used for the study of gaseous elements, the gas injector is often used in conjunction with the MEVVA to study metal ions. Injecting a low- $Z$ gas (usually nitrogen) helps higher- $Z$ ions attain higher charge states via a process known 


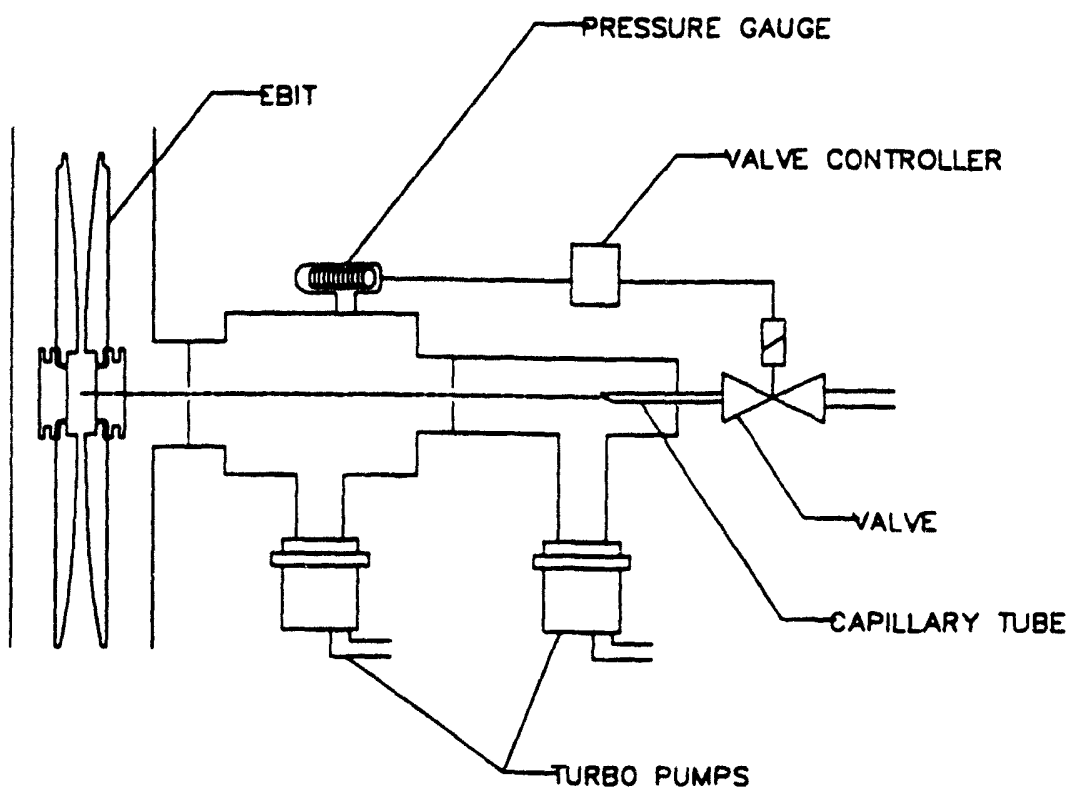

Figure 3.3: EBIT gas injecton system [80]. Two turbopumps reduce the pressure in the left chamber to approximately $10^{-7}$ torr, keeping the gas load on EBIT small. The desired pressure is set and maintained by a thermovalve which receives feedback from the pressure readings of the ionization gauge mounted on the left chamber. A capillary tube and two collimating holes direct a fine jet of gas through one of the slots in the drift tube assembly where it intersects with the electron beam. 
as "evaporative cooling." As described by Schneider et al. [76], at a given electron beam energy, although high- $Z$ ions will have lost a smaller fraction of their electrons than low- $Z$ ions, the average charge of the high- $Z$ ions will be much larger than the charge of the low- $Z$ ions. (For example, at $5000 \mathrm{eV}$, nitrogen will be completely stripped and have a charge of +7 , while iron ions will still have several of their 26 electrons left, but have an average charge of +20 or more.) Because of their higher charge, the high- $Z$ ions are trapped in a deeper energy well $\left(q_{\mathrm{i}} V_{\text {trap }}\right)$. Since ions in the trap undergo collisions with one another on timescales much shorter than electron-ion collisions, thus equipartitioning their energy, the lower- $Z$ ions are more likely to escape the trap, effectively carrying away excess energy from the higher- $Z$ ions and allowing them to undergo more ionizing collisions. Nitrogen cooling is extremely effective for high- $Z$ elements such as gold, which can have trapping times of hours or even days, but is of very little benefit for low- $Z$ elements such as neon.

EBIT has an extensive vacuum system, including roughing pumps, turbomolecular pumps, and ion pumps. There are also several valves that can isolate the electron gun and MEVVA to speed repairs or replacements. The gas injector and two of the six observation ports have gate valves; the other three ports are sealed with 5 -mil beryllium windows that hold off 1 atm pressure while still providing good $X$-ray transmission down to about $2 \mathrm{keV}$. Typical pressures inside the primary vessel are a few times $10^{-8}$ torr before the superconducting magnets are cooled with liquid helium. After cooling, the pressure drops below the $10^{-10}$ torr limit of the ionization gauges. Estimated pressures in the drift tube region are in the $10^{-12}$ torr range. By design, the surfaces in EBIT that are cooled by liquid nitrogen and helium act as cryopumps, greatly increasing the vacuum quality. Liquid helium is used only during experiment runs, but liquid nitrogen continuously cools EBIT's primary thermal shield. Frozen gases therefore accumulate on interior surfaces over time, including high voltage 
electrodes. This lowers the maximum energy at which the beam can be run, because of high voltage breakdown. Every few months, therefore, the entire cooling system must be shut down to let frozen contaminants evaporate and be pumped off.

\subsection{Operation}

Successful operation of EBIT requires a thorough understanding of its many components and the physical processes occurring in the trap as electrons and ions interact. Each measurement requires a different set of operating conditions, necessitating adjustment of the many voltages, currents, fields, and injection rates discussed above. A computer control system regulates many of those parameters, while also providing trigger pulses to a pair of data acquisition (DAQ) systems. The DAQ systems in turn record spectra accumulated by any spectrometers that are in use, as well as important beam parameters.

\subsubsection{Trap Parameters}

The most important physical parameter in EBIT is the electron-ion interaction energy because of its effect on which charge states are present and which atomic processes are excited. Although the ions can have considerable energy, up to hundreds of $\mathrm{eV}$, they are much more massive than the electrons and move much more slowly $\left(3.1 \times 10^{6} \mathrm{~cm} / \mathrm{s}\right.$ for a $200-\mathrm{eV}$ neon ion versus $1.9 \times 10^{9} \mathrm{~cm} / \mathrm{s}$ for a $1000-\mathrm{eV}$ electron). The interaction energy is therefore essentially equal to the electron beam energy, i.e., $e$ times the trap potential. The net voltage potential experienced by electrons passing through the trap is given by

$$
V_{\text {total }}=V_{D T}+V_{D T 2}-V_{\text {space }}
$$


where $V_{\mathrm{DT}}$ is the voltage on which all three drift tubes float, $V_{\mathrm{DT} 2}$ is the potential of the middle drift tube (bottom of the axial potential well), and $V_{\text {space }}$ is the spacecharge potential arising from the cumulative potentials of beam electrons, scattered electrons, and trapped ions. $V_{\text {space }}$ varies with distance from the center of the beam and provides radial confinement for the ions. The depth of the radial potential well is proportional to the amount of charge in the beam, as given by Gauss's Law.

If the electron beam is modeled as a uniform cylinder of charge, then the electron density is given by

$$
n_{e}=\frac{I}{e v_{e} A}=\frac{I}{e \sqrt{2 E / m_{e}} \pi r_{b}^{2}}
$$

where $I$ is the electron beam current, $e$ is the electron charge, $v_{e}$ is the beam velocity, $A$ is the beam area, $r_{\mathrm{b}}$ is the beam radius, and $E$ is the electron beam energy. Gauss's Law can then be applied to find the potential at the edge of the beam, given by

$$
V_{\text {space }}\left(r_{b}\right)=\frac{0.485 I}{\sqrt{E}} . \quad[\text { volis] }
$$

where the beam current is in $\mathrm{mA}$ and the energy is in $\mathrm{keV}$. In reality, the EBIT beam has a Gaussian density profile with a radius (containing $80 \%$ of the beam current) of approximately $35 \mu \mathrm{m}$ which is essentially independent of energy and current [32]. This makes the actual space charge potential slightly higher than given by Eq. 3.3. Trapped ions decrease the space charge, but only by a few percent. At $1 \mathrm{keV}$ and with $50 \mathrm{~mA}$ of current, $V_{\text {space }}$ is $25 \mathrm{~V}$ at the edge of the beam and decreases to zero at the center, introducing a spread in the ion-electron interaction energy. The total energy spread of the beam is somewhat larger because of transverse electron velocity (discussed in Chapter 6) and ripple in the high voltage power supply (approximately 
$20 \mathrm{~V}$ peak-to-peak). The total energy spread is typically $40 \mathrm{eV}$, but can be larger for high currents and low energies.

To find the potential difference between the center of the beam and the drift tubes (see Eq. 3.1), we again apply Gauss's Law, which yields

$$
V_{\text {space }}\left(r_{D T}\right)=V_{\text {space }}\left(r_{b}\right)\left[1+\ln \left(\frac{r_{D T}}{r_{b}}\right)\right]=6.8 V_{\text {space }}\left(r_{b}\right)
$$

where $\mathrm{DT}$ is the inner radius of the middle drift tube $(5 \mathrm{~mm})$. At $1 \mathrm{keV}$ and a current of $50 \mathrm{~mA}$, the potential difference between the center of the beam and the inner edge of the drift tube is approximately $170 \mathrm{~V}$ (see Eq. 3.1). The best way to accurately determine the space-charge potential and the energy spread of the beam is to vary the drift tube voltage to find a DR resonance of known energy and measure the FWHM (in energy) of the resonance.

As stated earlier, the space-charge potential of the beam provides radial confinement for the ions. Ions of charge $q_{i}$ with kinetic energy less than $q_{i} V_{\text {space }}\left(r_{\mathrm{b}}\right)$ will remain trapped inside the electron beam. Most ions have a higher kinetic energy, so that on average, ions spend only about $10-50 \%$ of their time actually in the electron beam. As long as their kinetic energy is less than $q_{i} V_{\text {space }}(r \mathrm{DT})$, however, ions will not be able to escape the trap by reaching the drift tubes. Additional confinement is provided by the magnetic field. For this reason, most ions escape from the trap along the axis rather than by striking the drift tube walls.

For a given ionization balance, the primary determinant of the number and distribution of the trapped ions is the shape and depth of the potential well, in both the axial and radial directions. Since the relative potentials of the top, middle, and bottom drift tubes are generally fixed, the character of the well is determined by the spacecharge potential. As can be seen in Equation 3.3. $V_{\text {space }}$ can be held constant if $I$ is varied as $E^{1 / 2}$. In that case, the electron density (and therefore the electron-ion 
overlap) will also be constant. This is an important point that will be discussed again in Chapter 5.

\subsubsection{Control System and Relevant Timescales}

Several EBIT subsystems, such as the gas injector, electron gun focus, magnetic steering coils, and the snout and transition electrodes, must be adjusted manually. Once they are set, they generally need only minor (though frequent) tuning. For the drift tubes and other subsystems that operate on millisecond timescales, however, an IBM XT computer serves as EBIT's electronic control system. Working through a CAMAC interface, the control program adjusts the voltage supplied to the anode and drift tubes while also providing triggers and gates for the data acquisition systems. The control program uses a graphical interface with separate screens for setting voltage and timing patterns.

Thirty-two channels are available for timing control, with four devoted to the common drift tube voltage, three channels for the three drift tube pieces, and two for the anode voltage (which controls the beam current). Additional channels can be assigned to anode or drift tube control as needed. The total voltage applied to the drift tubes is equa' to the sum of all drift tube voltages (set in the voltage control screen) that are "on" at that time (as determined by the timing pattern). Anode voltages are also fed into a summing amplifier before being sent to the anode power supply. Most of the other channels are used for triggers or gates to synchronize data acquisition with the drift tube voltage cycle.

An example of a timing pattern (showing only 12 of the most important channels) is shown in Figure 3.4. It can be broken into four primary sections. First is the dump phase, in which the top drift tube potential is lowered below that of the 
Clock period $=2.0 \mathrm{~ms}$; Total period $=4.5960 \mathrm{sec}$

20
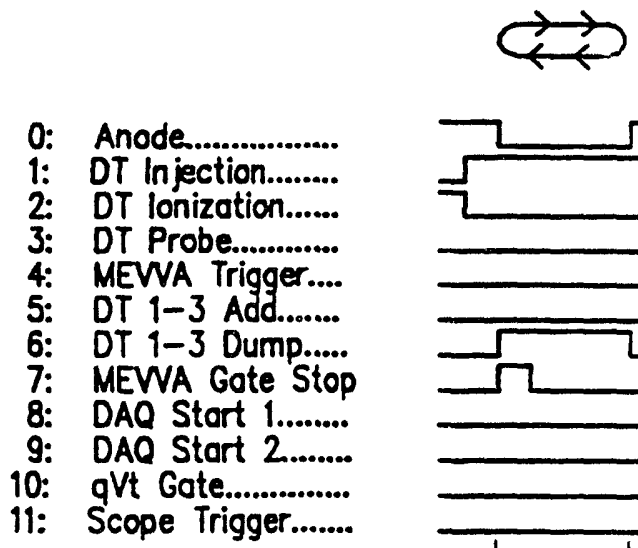

120
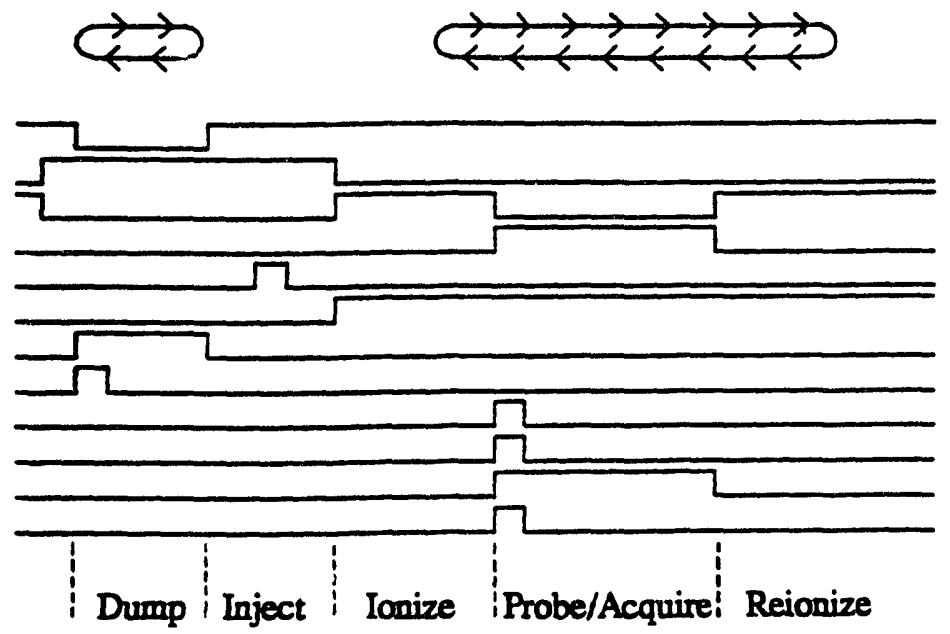

Figure 3.4: Example timing pattern for EBIT control program. Anode and drift tube voltages are set in a second screen, and controlled by the timing pattern. In each time step, a channel can be on (up) or off (down). Loops cause a sequence of time steps to repeat. There are four primary phases. (1) Dump ions out of the trap by lowering the top drift tube voltage. (2) Inject ions into the trap while it is open by firing the MEVVA and then raising the top drift voltage to trap the new ions. This stage is not used when the gas injector provides the ions. (3) Ionize the ions up to the desired charge state. (4) Acquire data. In this example, the drift tube voltage is switched between a "probe" voltage and the "ionization" voltage. Data are acquired during the probe phase, while the ionization phase reestablishes the charge balance. 
middle drift tube so that ions empty out of the trap. When the MEVVA is in use, the dump is immediately followed by the injection phase, in which the drift tube potentials are adjusted to admit and then trap a new batch of ions. This dump/injection procedure is needed to replenish the supply of ions in the trap, as they continually escape. Trapping times range from a few seconds for low-Z elements to hours or even days for the heaviest ions (with nitrogen cooling). If the target ions are provided instead by the gas injector (which continuously replenishes the trap with ions), the trap must still be dumped periodically to remove contaminants which build up over time, such as barium and tungsten from the electron gun. The trap must be dumped more frequently (on the order of every 10 seconds) when low- $Z$ ions such as neon are being studied, because they act as coolants for the heavier contaminant ions.

Next is the ionization phase, in which the desired charge balance is established. For low- $Z$ elements this takes only a few milliseconds, but for high- $Z$ ions with many electrons it can take a second or more. For the specific case of neon, the cross section for ionization of a Li-like ion is $2.7 \times 10^{-19} \mathrm{~cm}^{2}$ at $1000 \mathrm{eV}$. (Cross sections for ionization and recombination quoted in this section are taken from Arnaud and Rothenflug [3].) At a density of $4 \times 10^{12} \mathrm{~cm}^{-3}$ and an electron velocity of $1.9 \times 10^{9} \mathrm{~cm}$ $\mathrm{sec}^{-1}$ (the velocity at $1000 \mathrm{eV}$ ), the ionization rate $n_{e} v_{e} \sigma$ is approximately $2000 \mathrm{sec}^{-1}$, corresponding to a timescale of $0.5 \mathrm{msec}$. It also, of course, takes time to ionize from a neutral atom to the $\mathrm{Li}$-like charge state. At energies of a few $\mathrm{keV}$, above the ionization threshold of He-like neon $(1196 \mathrm{eV})$, the timescale for ionization of Li-like ions remains roughly the same, and ionization of He-like ions takes approximately 3 msec.

Competing with ionization is recombination, which generally proceeds more slowly. The cross section for radiative recombination, which scales as roughly $E^{-2}$, is $8.0 \times 10^{-23} \mathrm{~cm}^{2}$ for $\mathrm{H}$-like $\rightarrow \mathrm{He}$-like neon recombination at $1000 \mathrm{eV}$, corresponding to 
a timescale of about 1.5 seconds. Recombination of He-like ions takes about twice as long. Dielectronic recombination only occurs at discrete energies below threshold, but proceeds much more quickly. A typical resonance strength of $10^{-19} \mathrm{~cm}^{2} \mathrm{eV}$ averaged over the approximately $50-\mathrm{eV}$ energy width of the electron beam gives an effective cross section of $2 \times 10^{-21} \mathrm{~cm}^{2}$, which is a factor of 15 higher than for radiative recombination of $\mathrm{H}-\mathrm{like}$ ions at $750 \mathrm{eV}$. The timescale for dielectronic recombination ranges from tens to a few hundred msec, depending on the particular resonance.

Charge exchange recombination does not involve the beam electrons, and depends instead on the density of neutral gas atoms. A more detailed discussion of charge exchange is presented in Chapter 8 , but a rough estimate of the CER timescale is presented here assuming a cross section of a few times $10^{-15} \mathrm{~cm}^{2}$, an ion velocity of a few times $10^{6} \mathrm{~cm} \mathrm{sec}^{-1}$, and a neutral gas density of $10^{9} \mathrm{~cm}^{-3}$, giving a timescale of order $100 \mathrm{msec}$. At most electron beam energies, therefore, CER is the primary recombination mechanism.

The final stage of a timing cycle, after the ions have reached equilibrium and are ready for study, is the data acquisition (DAQ) phase, in which the electron beam energy is set at the desired "probe" energy and spectra are collected. If the probe energy is different frorn the "ionization" energy, the charge balance can be maintained by chopping the beam up and down in energy and setting the DAQ gates so that spectra are only acquired at the probe energy. Switching times for the beam energy are generally less than a msec, with a slew rate of approximately $10 \mathrm{keV} / \mathrm{msec}$, determined by the drift-tube high-voltage power supply.

Once ionization equilibrium has been attained, the most important cross sections during the data acquisition phase are obviously those for line excitation. Cross sections for excitation of the neon $K_{\alpha}$ lines are of order $10^{-20} \mathrm{~cm}^{2}$ near threshold, corresponding to timescales of approximately $10 \mathrm{msec}$. At higher energies, the cross 
sections for $x y$ and $z$ are much smaller, decreasing as $E^{-a}$, with $a$ between 2 and 3 . The typical timescale for radiative decay of excited ions is on the order of picoseconds $\left(10^{-12} \mathrm{sec}\right)$, so the chance of an excited ion undergoing further collisions before decaying is negligible. An exception is the highly forbidden transition from the $1 s 2 s$ ${ }^{3} S_{1}$ state that decays to produce line $z$. The cross section for collisional depopulation of that metastable state is roughly $10^{-18} \mathrm{~cm}^{2}$ at $1000 \mathrm{eV}$, corresponding to a timescale of order $0.1 \mathrm{msec}$, which is comparable to the radiative lifetime of that level.

The DAQ phase is generally the longest part of the timing cycle in order to maximize data collection efficiency. As mentioned before, cycle times are usually determined by the timescales for ion escape or for contaminants in the trap to reach unacceptable levels. A cycle time of 8 seconds was used to obtain most of the neon data. The unit time steps in the timing pattern are adjustable and can be as short as 0.1 msec, but are usually set to $1 \mathrm{msec}$. The choice is based on whether or not the beam energy is varied during the DAQ phase, and, if so, on the timescales for ionization and recombination.

\subsubsection{Data Acquisition Systems}

Since EBIT has five available observation ports, a number of spectrometers and detectors may be used simultaneously. Each has its own signal processing electronics, using gate and delay generators, time-to-analog converters, level discriminators, and so on. Processed signals are fed into analog-to-digital converters (ADCs) or multichannel analyzers (MCAs) which are themselves subject to coincidence requirements such as "beam on" (in case of beam crashes), successful MEVVA firings (if appropriate), and "DAQ gate on" (determined by the control program timing pattern). 
EBIT has two data acquisition systems: the original EBIT data acquisition system, known as EBDAQ, and the recently developed Event Mode System. In EBDAQ, the ADC and MCA outputs are fed into a CAMAC-based histogramming memory which is periodically read by the computer running the EBDAQ program. The program can display spectra or pulse height data from any of the spectrometers in use and also offers some basic analysis tools such as count summing, rebinning, and energy calibration. Data can be written to the hard disk or retrieved for analysis at any time, even while data are being collected.

A very important feature of EBIT is the ability to produce ions of a desired charge state at one energy, and then to probe the ions at a second beam energy, which is accomplished by rapid switching of the beam accelerating potential. With EBDAQ, data can be collected for one beam energy at a time, which is usually sufficient but does not make use of the time spent at other energies. With this in mind, the Event Mode system was developed to record the instantaneous time and electron beam energy for every detected photon, permitting spectra to be collected as the beam energy is varied continuously over a desired range. In this mode of operation, a waveform generator provides the signal (usually a triangle wave) that controls the drift tube voltage, sweeping the beam energy up and down at a rate fast enough to keep the ionization balance from changing. By recording and displaying spectra as a function of beam energy, the Event Mode system adds another dimension to cross section measurements. He-like neon data obtained in this manner will be discussed in detail in Chapter 8. 


\section{Chapter 4}

\section{Soft X-Ray Crystal Spectrometer}

EBIT was designed to study $\mathrm{X}$-ray emission from highly charged ions, so most of its spectrometers were designed for use at energies above a few keV. Several Bragg crystal spectrometers are available using flat crystals, or curved crystals in the Johann and von Hámos geometries. Bragg crystal diffraction is based on the scattering of X-rays from lattice planes in the crystal. If the distance between planes is $d$, constructive interference occurs when

$$
m \lambda=2 d \sin \theta_{B},
$$

where $m$ is the diffraction order (an integer), $\lambda$ is the wavelength of the diffracted radiation, and $\theta_{B}$ is the angle of reflection (Bragg angle) with $90^{\circ}$ being normal to the crystal surface.

Because X-rays are so easily absorbed in air, the EBIT crystal spectrometers are usually operated in a helium atmosphere (supplied by boil-off from the superconducting magnets) because of its much lower absorptivity. The detectors used in these spectrometers are all position-sensitive proportional counters with onedimensional imaging capability, sealed with beryllium windows 5 to 50 mils thick. (One mil is $0.001 "$ or $25.4 \mu \mathrm{m}$.) Spectral resolving power depends, among other things, on the type of crystal used and ranges from $\lambda / \Delta \lambda=500$ to 4500 . Because the ion-electron interaction region in EBIT is only about 70 microns wide, EBIT is essentially a line source so that no further apertures or slits are necessary.

Several solid state spectrometers using Ge or Si are also available. Their grasp is orders of magnitude higher than that of the crystal spectrometers but their energy 
resolution is much poorer, only about $100 \mathrm{eV}$ at $1 \mathrm{keV}$. Because of this and their large bandwidth, they are used as survey instruments to help tune EBIT or normalize data obtained with the crystal spectrometers. A lithium-drifted silicon, or $\mathrm{Si}(\mathrm{Li})$, detector was chosen for this neon experiment because it had a thin boron nitride window with good low-energy X-ray transmission.

The lower limit on the energy of X-rays that can be studied with the crystal spectrometers is set by the thickness of the Be windows on EBIT and the detectors. For a total path length of 10 mils ( 5 mil window on EBIT plus a minimum of 5 mils for the detector window), the $\mathrm{X}$-ray transmission of $\mathrm{Be}$ is $37 \%$ at $3 \mathrm{keV}, 17 \%$ at 2.5 $\mathrm{keV}$, and less than $4 \%$ at $2 \mathrm{keV}$. At $900 \mathrm{eV}$, the transmission is less than one part in 108. In addition, the absorption length for $900 \mathrm{eV}$ photons is less than $2 \mathrm{~mm}$ in air and about $16 \mathrm{~cm}$ in pure He. To study the X-ray emission from He-like neon, a new spectrometer had to be constructed.

There were several important design constraints on the new spectrometer. First, it had to be able to resolve the neon $K_{\alpha}$ lines. The two closest lines are $z$ and the $\mathrm{Li}$ like $q r$ blend, separated by $3.2 \mathrm{eV}$. To make sure that the two lines could be resolved even when one was significantly more intense than the other, a resolution of $2 \mathrm{eV}$ was desired, corresponding to a resolving power $\lambda / \Delta \lambda$ of approximately 460 . Next, the spectrometer required high throughput, with good low-energy $\mathrm{X}$-ray transmission through any windows (separating the spectrometer from EBIT or sealing the detector) and high detector quantum efficiency. It also needed to operate under vacuum and be inexpensive to build. A schematic of the resulting instrument, a vacuum Flat Crystal Spectrometer (FCS), is shown in Figure 4.1. Essentially it consists of a diffraction crystal and detector which are independently rotatable about a common pivot, inside a vacuum-tight box. 


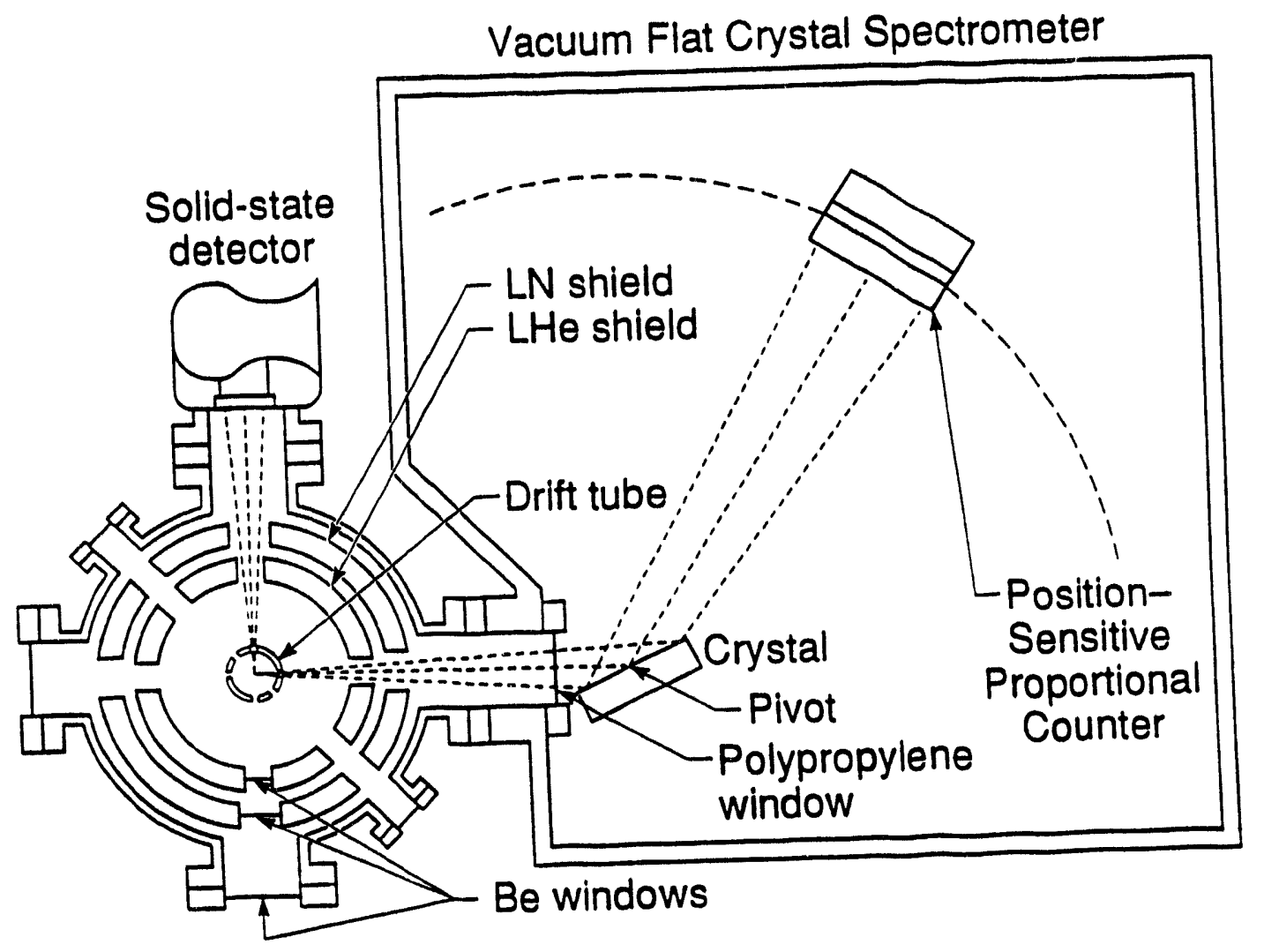

Figure 4.1: Schematic layout of the vacuum Flat Crystal Spectrometer. The electron beam is directed out of the page. The crystal and PSPC detector rotate about a common pivot point. The spectrometer is attached to one of EBIT's six observation ports, and uses a $1-\mu \mathrm{m}$ polyimide window to separate its vacuum from that of EBIT. 


\subsection{Diffraction Crystals}

Most of the He-like neon data were collected using TAP crystals (Thallium Acid Phthalate, chemical formula $\left.\mathrm{C}_{6} \mathrm{H}_{4}[\mathrm{COOH}][\mathrm{COO}] \mathrm{Tl}\right)$ which have the highest reflectivity of any crystal usable at wavelengths near $13.6 \AA$. With a planar spacing of $2 d=$ $25.76 \AA$ [40], the Bragg angle required for diffraction of He-like neon lines is about $32^{\circ}$. The intrinsic resolving power of TAP crystals at these wavelengths is about $\lambda / \Delta \lambda=600$. RAP (Rubidium Acid Phthalate) and KAP (Potassium Acid Phthalate) crystals have similar $2 d$ spacings and higher resolving power, but significantly lower reflectivity. Mica crystals ( $2 d=19.84 \AA$. $\theta_{B}=43^{\circ}$ for He-like neon $K_{\alpha}$ lines) are also widely available and have good resolving power ( $\lambda / \Delta \lambda$ approximately 1400$)$, but their reflectivity is less than $10 \%$ that of TAP. Apart from the acid phthalates and mica, there are virtually no other crystals to use in this wavelength region.

The flat crystal configuration was chosen because of its simplicity. Although curved crystals generally have higher collection efficiency because of their focusing properties and have been used with success on EBIT, particularly in the von Hámos spectrometer [11], proper alignment of the crystal and detector is a time-consuming and exacting procedure which would be very difficult to accomplish under vacuum. Flat crystals are by comparison easy to align, and also have larger bandwidth and lower cost. In addition, the EBIT research group has several kinds of flat crystals, all of which are the same size (1" by $2 ")$ and can be easily interchanged.

\subsection{Proportional Counter}

The FCS uses a single-wire 1-D position-sensitive proportional counter (PSPC) to detect X-rays dispersed by the crystal. Position information is obtained using the 
rise-time, or resistance-capacitance, method described by Borkowsi and Kopp [16]. The detector was originally sealed with a Be window, but was modified to detect lowenergy $\mathrm{X}$-rays for this experiment. The new window consists of a $4-\mu \mathrm{m}$ thick polypropylene $\left(\mathrm{CH}_{2}\right)_{x}$ foil coated with approximately $150 \AA$ of aluminum for electrical conductivity. The $80 \mathrm{~mm}$ by $8 \mathrm{~mm}$ open area is supported by a 1 -mm-wide bar down the length of the window. To mount the window, a 1/64" thick Viton gasket is placed on top of the face of the detector body, followed by the polypropylene film (Al-coated side down), and finally the ribbed window support frame. The window is then sealed by tightening two rows of screws attaching the window frame to the detector body.

Extensive tests of the window assembly showed that the polypropylene can be safely pressurized to about $5 \mathrm{~atm}$. Although there is virtually no chance of a properly mounted window rupturing under vacuum while operating with a gas pressure of 1 atm, the window must still be replaced every few months because of fatigue caused by repeated cycling of the vacuum. Microscopic perforations gradually appear in the window and are manifested by a decrease in the quality of the vacuum.

The 4- $\mu \mathrm{m}$ polypropylene film was chosen for several reasons. Other window materials such as lexan $\left(\mathrm{C}_{6} \mathrm{H}_{14} \mathrm{O}_{3}\right)$, paralene $\left(\mathrm{C}_{8} \mathrm{H}_{8}\right)$, and mylar $\left(\mathrm{C}_{10} \mathrm{H}_{8} \mathrm{O}_{4}\right)$ have higher mass absorption coefficients and therefore need to $h \mathrm{c}$ thinner than polypropylene to achieve the same X-ray transmissivity. Since a rupture of the PSPC window would be disastrous for EBIT, strength (and therefore thickness) was an important consideration. In addition, unlike any of the other materials, the polypropylene was available in large quanuies of the desired thickness at very reasonable prices (a few hundred dollars for thousands of square feet) and came already coated with aluminum. (The manufacturer uses it to make capacitors.) 


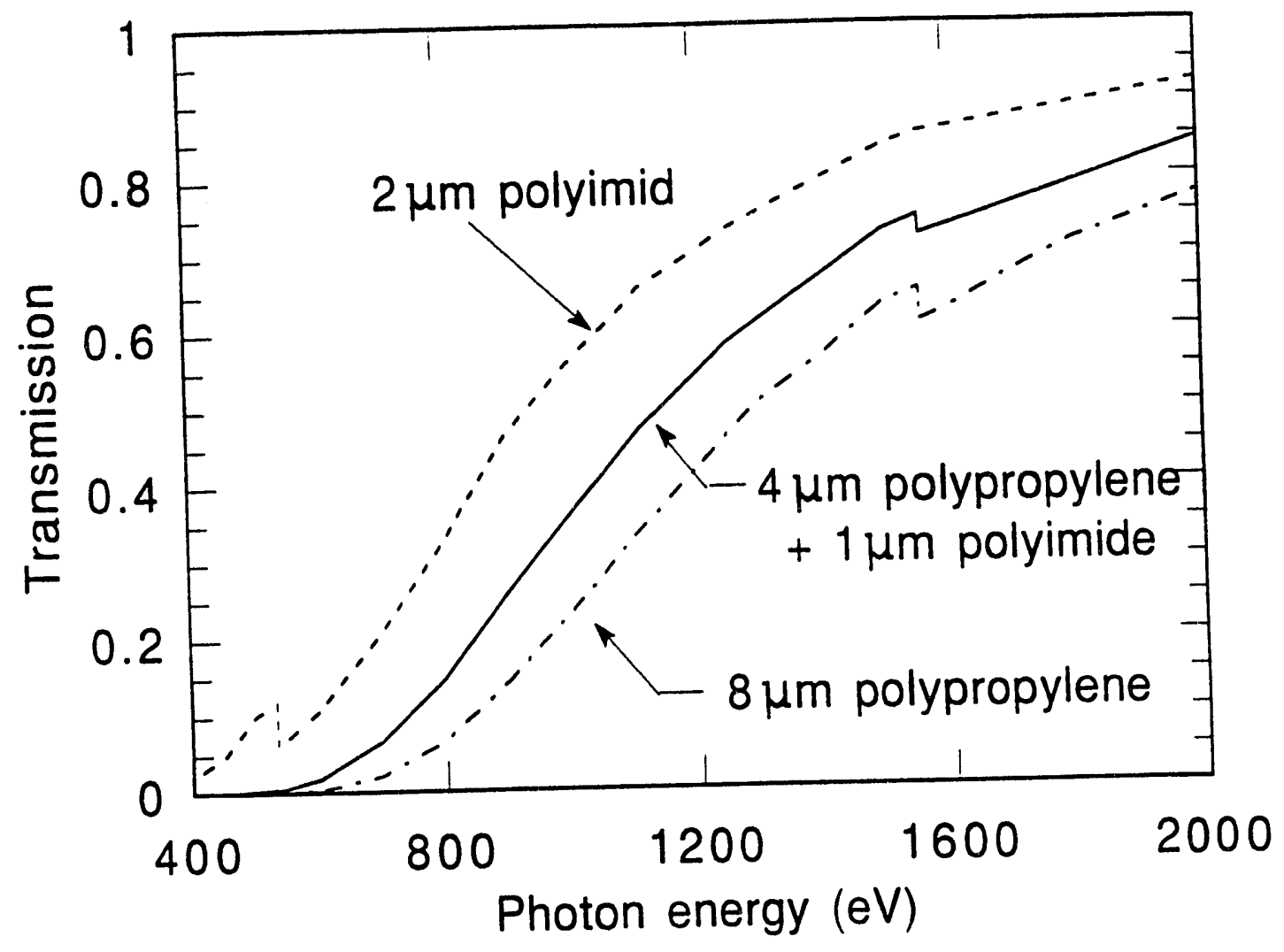

Figure 4.2: X-ray transmission versus photon energy for three combinations of two thin plastic windows. Windows are composed of either $4-\mu \mathrm{m}$ polypropylene or $1-\mu \mathrm{m}$ polyimide, each coated with $150 \AA$ of aluminum. 
Polypropylene was also used in the window separating the spectrometer vacuum (typically $2 \times 10^{-6}$ torr) from that of EBIT. Without this window the EBIT trap vacuum (approximately $10^{-12}$ torr) and therefore the charge balance would be severely degraded. During the early stages of this project, the spectrometer window was replaced by a $1-\mu \mathrm{m}$ thick polyimide foil $\left(\mathrm{C}_{22} \mathrm{H}_{10} \mathrm{O}_{4} \mathrm{~N}_{2}\right)$ which had higher $\mathrm{X}$-ray transmission and a lower gas diffusion rate. Quality polyimide foils are difficult to produce, however, and are not available in sizes larger than about 2 inches in diameter. Putting a polyimide window on the PSPC would therefore require a more complex, costly, and supportive windowpane arrangement. The transmission of various combinations of polyimide and polypropylene windows is shown in Figure 4.2. The 4- $\mu \mathrm{m}$ polypropylene $/ 1-\mu \mathrm{m}$ polyimide combination used during most of the project had a net transmission of about $25 \%$ at $920 \mathrm{eV}$.

Thin plastic windows are much more permeable than Be foils and the counter gas slowly leaks through the window. This necessitated converting the detector, which was originally permanently sealed, into a "flow counter" with a continuous gas supply. Two holes were drilled through the detector body into the gas chamber, and gas fittings attached. Two flexible metal tubes connect the PSPC gas fittings to another set of fittings on a "feed-through" flange on the spectrometer housing. Electrical feed-throughs supply connections for the high voltage, power, and signal cables. P10 gas, a mixture of $90 \%$ argon and $10 \%$ methane well-suited for detection of low energy $\mathrm{X}$-rays, is supplied at a pressure of just over $1 \mathrm{~atm}$ by a gas cylinder with a precise low pressure regulator. At the exhaust end, gas is bubbled through a cup of water to keep the pressure constant and provide information on the rate of flow.

The net resolution of the spectrometer depends upon detector spatial resolution, crystal resolving power, spatial extent of the source, etc. Since the EBIT source width is only $70 \mu \mathrm{m}$, the crystal and detector resolution are the most important factors. For 
Bragg diffraction, the spectrometer resolving power is given by the quotient of the Bragg equation and its derivative,

$$
\frac{\lambda}{\Delta \lambda}=\frac{\tan \theta}{\Delta \theta},
$$

where $\theta$ is the angle of diffraction and $\Delta \theta$ is the net angular width of a detected line. Spatial resolution of the detector depends on the X-ray energy and anode voltage (which must be adjusted to provide the best signal height), but at $900 \mathrm{eV}$ the resolution is about $250 \mu \mathrm{m}$. The combined source-to-crystal-to-detector distance in the FCS is $50 \mathrm{~cm}$, so this provides a resolving power of 2000 at $45^{\circ}$. For neon $K_{\alpha}$ spectra, spectrometer resolution is therefore limited by the intrinsic resolving power of the $\operatorname{TAP}(\lambda \Delta \lambda \approx 600)$ and mica $(\lambda \Delta \lambda \approx 1400)$ crystals.

\subsection{General Layout}

The spectrometer vacuum vessel was designed to put the crystal as close as possible to EBIT to maximize X-ray flux, and then, given that constraint, to maximize the range of allowable Bragg angles for maximum wavelength coverage. With a gate valve between the EBIT observation port and the spectrometer (required so the spectrometer can be brought up to air or removed without affecting EBIT), the center of the crystal is 11.5 " away from the beam. The PSPC is located 9" from the crystal, in a compromise between optimizing flux and spectral resolution. Bragg angies up to $63^{\circ}$ are accessible, meaning that the $K_{\alpha}$ lines of He-like oxygen $\left(\theta_{B} \approx 59^{\circ}\right.$ with TAP crystals) can be observed.

The crystal and PSPC both rotate about a common pivot on concentric axles that extend through a hole in the bottom of the spectrometer box to the outside. Rubber $\mathrm{O}$ rings, sliding on Teflon sleeves, seal the axles against one another and the box frame. 
This arrangement allows pointer arms on the outside of the box to adjust the crystal and PSPC angles while the spectrometer is under vacuum. Two steel pins on the crystal pivot are used to hold the crystal (attached to a mounting block with melted wax) in place as it rotates. The PSPC rotates about the same point at the end of a pivot arm which is attached to the PSPC mounting plate. The plate has a set of four roller bearings and moves along a curved track attached to the bottom of the spectrometer.

The spectrometer box is $25^{\prime \prime}$ long, $17.5^{\prime \prime}$ wide, and $6.75^{\prime \prime}$ tall. It is made of aluminum and was designed in three sections for ease of construction. The bottom plate is $3 / 4$ " thick and has a hole for the pivot axis, as well as four more 2.5 "-diameter holes along the back edge. One is for the vacuum pumps, and the others are sealed by feed-through flanges for the gas tubing, high voltage and signal cables, and powerpreamplifier cable.

Two steel pins align the bottom plate with the 4 " tall side wall section. This side section contains the $\mathrm{X}$-ray viewing port, which is connected to the gate valve on EBIT's observation port by a steel adapter flange. The spectrometer window, which separates the spectrometer and EBIT vacua, is mounted on the inside of the box directly over the X-ray port opening. A pump-around (not shown in Figure 4.1) can be valved open to keep the pressure on both sides of the window the same when the spectrometer is being pumped down or let up to air.

The lid is $2 "$ tall, and has been milled out to a thickness of $3 / 4$ " to allow enough room for the gas tubing which is connected to the gas fittings on top of the PSPC. The fittings are on top on the detector, rather than the sides, to avoid obstructing the movement of the PSPC at the largest and smallest Bragg angles. There are two ports on the top, one for an ionization pressure gauge and the other for a 6"-diameter Plexiglas window that allows direct observation of the spectrometer window, crystal, 
and PSPC. The Plexiglas can also be removed to access the crystal, rather than having to lift off the entire (heavy) lid.

Rubber O-rings are used to seal the window and all flanges, including the seals between the bottom plate, side walls, and lid. A turbopump backed by a roughing pump brings the pressure of the empty chamber down to below $10^{-7}$ torr. With the PSPC in place and running with P10 gas the pressure is a factor of ten higher because of diffusion through the detector window. A good vacuum is desired to minimize the gas load on EBIT, which must operate under ultrahigh vacuum. X-ray absorption by residual gas is completely negligible.

The FCS has been used with great success to study soft X-ray spectra on EBIT. In addition to the measurements discussed in this thesis, spectra have been ubtained of $L$-shell emission from neonlike iron (shown in Figure 4.3) and the Lyman series of hydrogenlike oxygen [6], to name just a few examples of astrophysical relevance. In the future, replacement of the $4-\mu \mathrm{m}$ polypropylene PSPC window with $1-\mu \mathrm{m}$ polyimide will permit the study of He-like oxygen. The PSPC has proven to be very versatile and reliable, and two more modified PSPCs have been constructed. In addition to acting as backups for the vacuum FCS, they have also been used in Heenvironment crystal spectrometers to study $\mathrm{X}$-rays down to $2.5 \mathrm{keV}$. Examples include the $K_{\alpha}$ spectrum of He-like sulfur and the first observation of a magnetic octupole line, in Ni-like thorium and uranium [10]. 


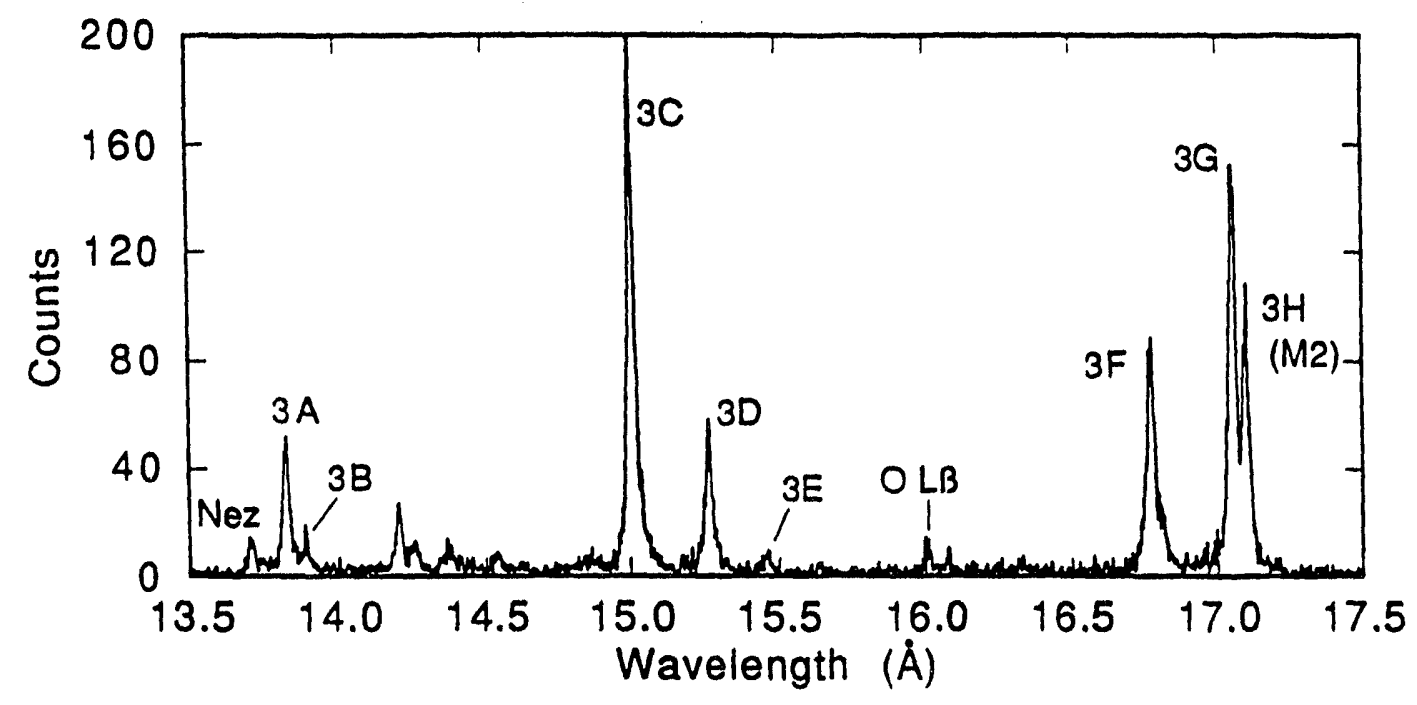

Figure 4.3: Spectrum of $3 \rightarrow 2$ lines in neonlike FexvII recorded by the Soft X-ray FCS. The spectrum is a composite of data from three spectrometer settings. The oxygen Lyman- $\beta$ and He-like neon $z$ lines arise from residual gas in EBIT. From Ref. [6]. 


\section{Chapter 5}

\section{Experimental Procedure}

As explained in Chapter 2, many processes and several ion stages contribute to the excitation of He-like 2-to-1 lines and their satellites. Chapter 3 introduced EBIT, an extremely powerful tool for investigating such processes, and Chapter 4 described the spectrometer used to study He-like neon. This chapter discusses the experimental procedures adopted to constrain the processes contributing to He-like neon $K_{\alpha}$ emission.

\subsection{Desired Measurements}

The desired end product of this experiment is a set of cross sections and rates for all the processes involved in production of lines $w, x, y, z$, and any blended satellite lines as a function of electron energy, density, and the fractional abundance of the ion stage involved. These cross sections and rates can then be assembled, using any electron energy distribution, density, or ionization balance, to compute line intensity ratios. The process can subsequently be inverted, using observed line ratios as diagnostics to deduce the temperatures, densities, and ionization states of astrophysical $\mathrm{X}$-ray sources.

Before discussing how the various cross sections and rates were measured, the reader may find it useful to briefly review the processes contributing to excitation of He-like $K_{\alpha}$ lines. The following discussion is divided into paragraphs which separately list the contributions of He-like, Li-like, and $\mathrm{H}$-like ions. 
For collisional plasmas, the most important cross sections are those associated with the electron impact excitation (EIE) of $w, x, y$, and $z$. Li-like dielectronic recombination (DR) satellites (whose intensities are proportional to the He-like ion abundance) often blend with the true He-like lines and can make significant contributions to their apparent intensities at low temperatures, particularly for lines $y$ and $w$. The intensity of all the $K_{\alpha}$ lines is enhanced by resonant excitation (RE), although that process is most important for $x y$ and $z$.

Line $z$ is also excited by innershell ionization (ISI) of Li-like neon. Innershell excitation produces the collisional satellites $q$ and $r$. As will be discussed in section 5.3, the relative cross sections for excitation of $z$ (via ISI) and $q r$ can be determined from observing how the ratio $I_{z} / I_{q r}$ changes as the relative abundance of $\mathrm{Li}$-like and He-like charge states is varied.

Ideally, the contribution of recombining $\mathrm{H}$-like neon would be measured, too. As discussed in Chapter 3, however, the rate of radiative recombination (RR) of $\mathrm{H}$-like ions is completely negligible compared to that for EIE of He-like neon, and is therefore impossible to measure on EBIT with any kind of accuracy. Dielectronic recombination (DR) rates are significantly larger than for $R R$, but only at discrete energies below the ionization threshold for production of $\mathrm{H}$-like ions. Charge exchange recombination (CER) is the dominant recombination mechanism in EBIT at most energies, but because only a small fraction of recombinations lead to excitation of the $K_{\alpha}$ lines (as discussed in Chapter 8), its contribution to line excitation is negligible except at very high energies or $\mathrm{H}$-like ion abundances.

All of these recombination mechanisms, except for DR, are difficult or impossible to measure on EBIT or any other device. From an astrophysical point of view, however, CER is of virtually no importance. $R R$ and $D R$ can be very important in exciting He-like $K_{\alpha}$ lines if the fraction of H-like ions is high, but because of the 
simplicity of the H-like system, theoretical estimates for these processes are accurate to a few percent.

Experimental measurements of the He-like and Li-like processes described above, combined with H-like theory, are sufficient to predict line intensity ratios at any temperature or nonthermal energy distribution for any ionization balance in the low density limit, that is, assuming that there are no collisions while an ion is in an excited state. As previously mentioned, however, the ${ }^{3} S_{1}$ state producing line $z$ is relatively long-lived, so that at high densities electron collisions can transfer the excited electron from $2^{3} S_{1}$ to $2^{3} P$ or other higher- $n$ levels. This competition between radiative and collisional depopulation is what gives rise to the density sensitivity of the line ratio $R=I_{z} \Pi_{x y}$. To calibrate this effect, the cross section for the relevant collisional transfer must be measured, along with the radiative decay rate of the metastab!z ${ }^{3} S_{1}$ level.

\subsection{Low Energy Measurements}

Table 5.1 lists the processes relevant to the production of He-like neon $K_{\alpha}$ lines, along with their respective energy ranges, the specific lines that are excited, and the ion stages involved. Contributions from Li-like neon only occur at energies above the innershell ionization threshold, which is slightly below the threshold for ionization of He-like to H-like because of the shielding effect of the extra $2 s$ electron. Likewise, at energies below the He-like ionization threshold (1196 eV), no H-like ions will be produced. This means that if EBIT's electron beam energy is kept below that threshold, there will be no $\mathrm{H}$-like ions to recombine. The cross section for innershell ionization of Li-like ions is negligibly small below $1196 \mathrm{eV}$ (see Chapter 8), so its contribution to the intensity of $z$ can also be ignored. The intensities of $w, x, y$, and $z$ 


\begin{tabular}{|c|c|c|c|}
\hline Process & $\begin{array}{c}\text { Energy } \\
(\mathrm{eV})\end{array}$ & $\begin{array}{c}\text { Lines } \\
\text { Produced }\end{array}$ & $\begin{array}{c}\text { Ion } \\
\text { Stage }\end{array}$ \\
\hline Ionization $\mathrm{He} \rightarrow \mathrm{H}$ & $>1196$ & $\ldots$ & $\mathrm{He} \rightarrow \mathrm{H}$ \\
\hline Electron Impact Exc. of $n=6-\infty$ & $>1165$ & $w^{\prime}, x^{\prime}, y^{\prime}, z^{\prime}$ & $\mathrm{He}$ \\
\hline Electron Impact Exc. of $n=5$ & $>1152$ & $w^{\prime}, x^{\prime}, y^{\prime}, z^{\prime}$ & $\mathrm{He}$ \\
\hline Electron Impact Exc. of $n=4$ & $>1127$ & $w^{\prime}, x^{\prime}, y^{\prime}, z^{\prime}$ & $\mathrm{He}$ \\
\hline Innershell Ionization $\mathrm{Li} \rightarrow \mathrm{He}$ & $>1107$ & $z$ & $\mathrm{Li} \rightarrow \mathrm{He}$ \\
\hline Electron Impact Exc. of $n=3$ & $>1073$ & $w^{\prime}, x^{\prime}, y^{\prime}, z^{\prime}$ & $\mathrm{He}$ \\
\hline Resonant Exc. KMN & $\sim 1020$ & $w, x, y, z$ & $\mathrm{He}$ \\
\hline Resonant Exc. KMM & $960-970$ & $w, x, y, z$ & $\mathrm{He}$ \\
\hline Electron Impact Exc. of $2^{1} P_{1}$ & $>922$ & $w$ & $\mathrm{He}$ \\
\hline Electron Impact Exc. of $2^{3} P_{2,1,0}$ & $>916$ & $x, y, z^{\prime}$ & $\mathrm{He}$ \\
\hline Electron Impact Exc. of $2^{3} S_{1}$ & $>905$ & $z$ & $\mathrm{He}$ \\
\hline 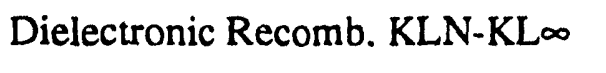 & $860-920$ & "w","y" & $\mathrm{He} \rightarrow \mathrm{Li}$ \\
\hline Dielectronic Recomb. KLM & $814-824$ & "w","y" & $\mathrm{He} \rightarrow \mathrm{L}$ \\
\hline Dielectronic Recomb. KLL & $667-682$ & "y",qr,"z" & $\mathrm{He} \rightarrow \mathrm{Li}$ \\
\hline Ionization $\mathrm{Li} \rightarrow \mathrm{He}$ & $>239$ & $\ldots$ & $\mathrm{Li} \rightarrow \mathrm{He}$ \\
\hline Collisional transfer $2^{3} S_{1} \rightarrow 2^{3} P_{2,1,0}$ & $>10$ & $x, y$ from $z$ & $\mathrm{He}$ \\
\hline
\end{tabular}

Table 5.1: List of all significant atomic processes related to the production of He-like neon $K_{\alpha}$ emission. A prime (') means that the line is excited via cascades. DR satellites are denoted by the He-like line with which they blend in quotes, e.g., the DR KLL satellite line $k$ is listed as " $z$ " because it is very close in wavelength to $z$ The Li-like satellite lines $q$ and $r$ are produced by innershell EIE with a threshold of $908 \mathrm{eV}$. 
(and their DR satellites) then depend on only He-like ions and their cross sections for electron impact excitation (EIE), dielectronic recombination (DR), and resonant excitation (RE). The Event Mode data acquisition system is perfectly suited for this situation, recording the $K_{\alpha}$ line intensities as the electron beam is swept up and down in energy, mapping out all the resonances and cascade jumps from the lowest DR resonances up to the He-like ionization threshold.

Running EBIT at such low energies, however, presented many challenges. In the normal operating mode, $V_{\text {anode }}<V_{\text {snout }}<V_{\text {transition }}<V_{D T}$ so that the beam electrons move smoothly and progressively faster on their way to the trap. Operating with $V_{D T}$ less than $1 \mathrm{kV}$ made this impossible, and prior to this experimental program, virtually no work had been done with drift tube voltages less than about $4 \mathrm{kV}$. Fortunately, EBIT proved to be much more amenable to low energy operation than expected, and after a short period of trial and error, a stable mode of operation was found for energies as low as about $600 \mathrm{eV}$. The beam can be run at even lower energies, but only with small currents since there appears to be an excluded operating region for beam currents between about 20 and $40 \mathrm{~mA}$ for all energies up to about $2000 \mathrm{eV}$. The ion-electron interaction rate scales as approximately $\beta^{3 / 2}$ in EBIT, so virtually all the He-like neon measurements were conducted using the higher current operating mode.

Another aspect of operating at low energies is that the beam energy spread (typically about $50 \mathrm{eV}$ FWHM) is proportionately larger, tending to smear out resonances and thresholds. Solid state spectrometers are also less effective, not only because their resolving power is worse at low photon energies but also because there is a significant low-energy background arising from bremsstrahlung and emission from residual gas (mostly oxygen and nitrogen but also higher- $Z$ contaminants). The combination of low resolving power and high background means that RR peaks 
cannot be resolved, and so their intensities cannot be used to normalize crystal spectrometer data.

For He-like neon, relative cross sections must instead be determined by normalizing line intensities (as seen in the FCS) to the amount of time spent at a given energy. For the low-energy measurement, this was accomplished by ramping the beam energy up and down using a triangular wave function so that an equal amount of time was spent collecting data in equal energy intervals. An important assumption in this method, however, is that conditions in the trap do not change as the beam energy is varied. Specifically, the number and distribution of ions in the trap must remain the same. Although ion conditions are of course affected by the gas injection rate, trap depth, and so on, the only factor that matters on the timescale of the beam energy ramp (of order one $\mathrm{msec}$ ) is the space charge, or equivalently, the density of electrons in the beam (see section 3.2.1). Another reason for keeping the electron density constant is that the rate of collisional transfer between ${ }^{3} S_{1}$ and the ${ }^{3} P$ levels is proportional to density.

As explained previously, to maintain a constant density the beam current must be varied as $E^{1 / 2}$. Based on the maximum current that could be run at the lowest energies, a baseline of $49 \mathrm{~mA}$ at $1 \mathrm{keV}$ was adopted. For a given electron gun filament temperature, the amount of current that can be extracted from the filament is simply proportional to the velocity of the electrons as they are pulled toward the anode, that is, $I_{\text {beam }} \propto \sqrt{V_{\text {anode }}}$. This theoretical relationship was experimentally confirmed to better than $1 \%$ accuracy over the entire range required, and meant that $V_{\text {anode }}$ could be controlled using the same triangular input signal (with proper scaling) as $V_{D T}$.

The low energy data were collected using the Event Mode system in two experimental runs. The first run included measurement of the lowest-energy DR 
satellites, in the KLL band around $670 \mathrm{eV}$. It extended up to the threshold for ionization of He-like neon near $1200 \mathrm{eV}$, but the rate at which the beam energy was varied $(1000 \mathrm{~Hz}$, or $1.1 \mathrm{keV} / \mathrm{msec})$ tended to smear out the details of how the cross section of $z$ varied with energy because of the nonnegligible lifetime of the $2^{3} S_{1}$ level. (See Chapter 7 for a description of how the radiative rate of $z$ was measured.) The second measurement covered the energy range from 750 to $1200 \mathrm{eV}$ at a lower rate, and did not include the KLL resonances since adequate statistics were obtained in the first run. Starting at a higher energy meant that a smaller energy range had to be covered and that a higher beam current could be used, both of which increased the data collection rate.

For the second measurement, a triangle wave with a frequency of $232 \mathrm{~Hz}$ controlled $V_{D T}$ and $V_{\text {anode }}$, corresponding to an energy slew rate of $104 \mathrm{eV} / \mathrm{msec}$. At that rate, the $91-\mu \mathrm{sec}$ lifetime for radiative decay of $2^{3} s_{1}$ corresponded to less than 10 $\mathrm{eV}$, much less than the roughly $50-\mathrm{eV}$ beam energy resolution, so that smearing of features in the excitation cross section of $z$ was negligible. At the same time, the ramp rate was sufficiently fast that the ionization balance remained virtually unchanged (ramp period $=4.3 \mathrm{msec}$ vs. ionization timescale $\sim 10 \mathrm{msec}$, but the cross sections for ionization and recombination are practically constant over the small energy range covered). A schematic of the voltage/timing pattern used is shown in Figure 5.1a. Data were collected in this way until approximately 400 counts had been accumulated in each 20-eV energy bin for the $x y$ blend, the weakest of the $K_{\alpha}$ lines.

\subsection{High Energy Measurements}

At energies above the threshold for ionization of He-like ions, the $K_{\alpha}$ EIE cross sections are smoothly varying, without any further resonances or cascade jumps, so 

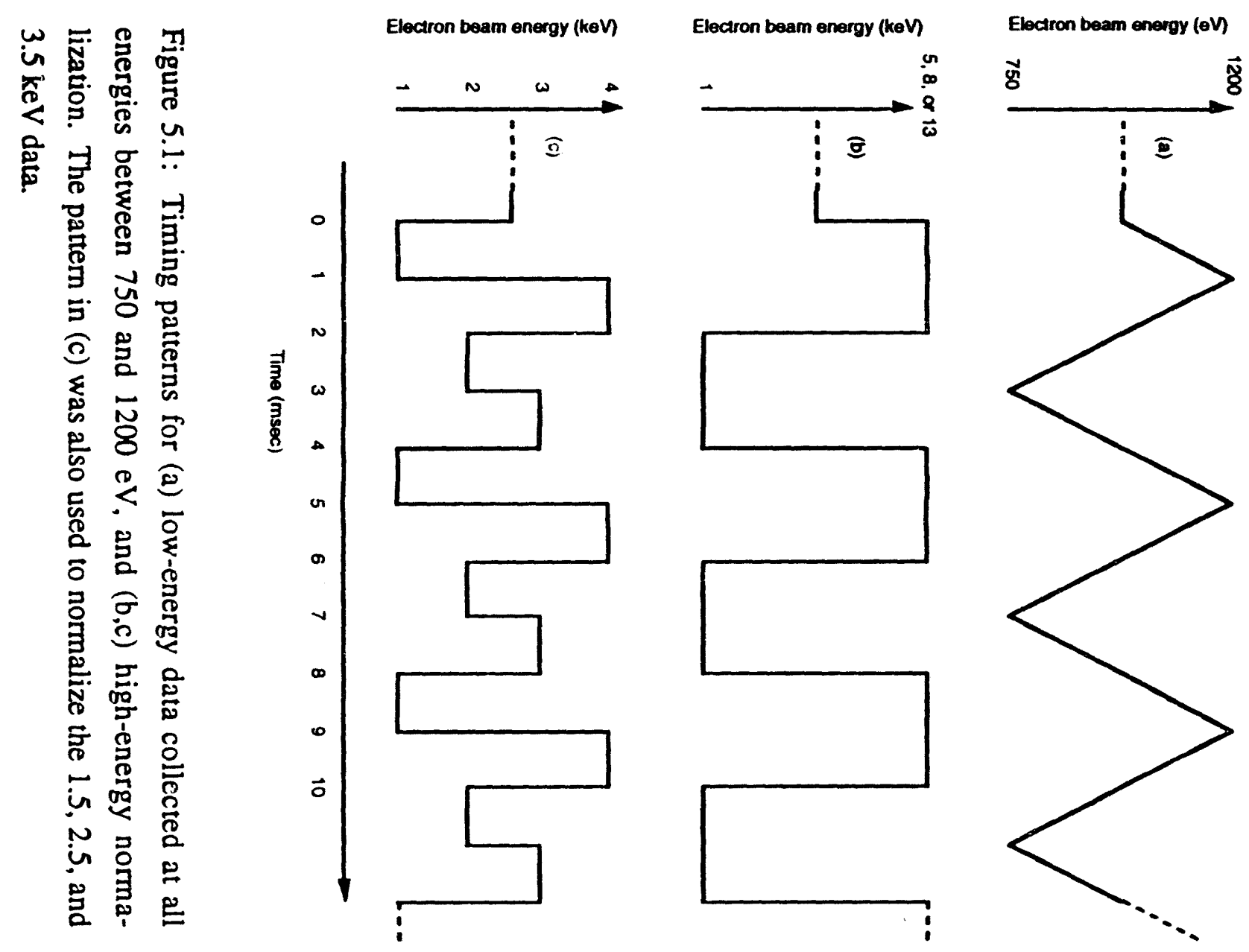
that only a few measurements at widely spaced energies are necessary. The Li-like ISI cross section is also smooth, rising from zero at its $1107-\mathrm{eV}$ threshold and increasing until it peaks at about two and a half times the threshold energy and then falling asymptotically as $\ln E / E$. The two contributions to the intensity of $z$, EIE of He-like and ISI of $\mathrm{Li}$-like ions, can each be determined by varying the ratio of $\mathrm{He}$-like to $\mathrm{Li}$ like abundance, using the intensities of lines $w$ (proportional to $n_{\mathrm{He}}$ ) and $q r$ (proportional to $n_{L i}$ ) for comparison. When $I_{q r}=0$, there is no ISI contribution and line $z$ is produced strictly by EIE, while for nonzero $n_{L i}$, any increase in $I_{z}$ is proportional to $I_{q r}$. The two contributions to the $x y$ blend, direct excitation by EIE and collisional transfer from line $z$, can be similarly determined.

There were three primary concerns in making this set of measurements. First, the ratio of Li-like and He-like ion density, $n_{L i} / n_{H e}$, had to be varied over as wide a range as possible to achieve the best results. The density of H-like ions, $n_{H}$, had to, at the same time, be minimized so $t^{2}$ ombination-induced $K_{\alpha}$ emission was negligible. Lastly, there had to $b \in$ vay of normalizing the cross sections obtained at different energies.

One way to increase the fraction of Li-like ions is to set the electron beam energy on a He-like DR resonance. This is much more effective than setting the beam energy below the threshold for Li-like ionization because cross sections for DR (at resonance) are orders of magnitude larger than for RR. (It is also impossible to run the beam at energies below the $239-\mathrm{eV}$ ionization threshold for Li-like neon.) Once the charge balance is established, the beam energy can be raised above the ISI threshold for a short time to collect data and then be lowered back to the resonance to replenish the $\mathrm{Li}$-like fraction. This method is most effective for higher- $Z$ elements, and has been used very successfully to study ISI in Li-like $\mathrm{Cr}^{21+}$ and $\mathrm{Fe}^{23+}[80]$. 
For lower- $Z$ elements such as neon, however, the cross sections for recombination in general and DR in particular are relatively weak. A iarger problem is that the He-like neon DR resonances are at very low energies where EBIT is most unstable, so that jumping from the $K L L$ resonance complex around $670 \mathrm{eV}$ up to several $\mathrm{keV}$ to make ISI measurements is likely to make EBIT crash.

Instead, the best way to produce a high fraction of Li-like neon ions is to use high gas injection rates with a shallow trap potential, in this case only $80 \mathrm{~V}$ instead of the usual 200 or $300 \mathrm{~V}$. The shallow well means that ions are only loosely bound within the trap) and are therefore more likely to escape before reaching highly charged states. The high gas injection rate also acts to lower the average charge state in the trap, mostly through charge exchange recombination. (There is no evaporative cooling effect here because there is no coolant gas, with $Z$ less than that for neon, being injected.)

High-energy measurements were made at nine energies: $1.5,2.0,2.5,3.0,3.5$, $4.0,5.0,8.0$, and $13.0 \mathrm{keV}$. In terms of energy coverage (in units of threshold energy), this was by far the most extensive investigation ever conducted on EBIT, measuring cross sections from the lowest DR resonance up to 14 times the EIE threshold. At each energy, spectra were collected with several (at least 5) different ion abundance ratios, with $n_{L i} / n_{H e}$ typically varying by a factor of 4 or 5 as the gas injection pressure was varied by a factor of up to 100 . At the low-pressure end, the limiting factor was the need for reasonable data rates (the number of ions was of course smaller at low gas pressures) and the fact that a completely pure charge state can never be attained in EBIT, while at the high-pressure end, the electron beam became unstable if the gas injection rate was raised too high. As with the low energy data, the beam current was adjusted to maintain a constant electron density at all energies. 
Most of the measurements were made with the ions in steady state, that is, with the beam energy and current held constant. Spectra were recorded using EBDAQ. In order to normalize those measurements so that relative cross sections at different energies could be computed, at least one measurement at each energy was made by switching the beam energy up and down between the high energy and $1 \mathrm{keV}$. The baseline energy of $1 \mathrm{keV}$ was chosen because the excitation cross sections of all $\mathrm{K}_{\alpha}$ lines are relatively flat at that energy, with no resonances or jumps from cascades (see Table 5.1). The high energy cross sections could also be normalized to the continuous-coverage low energy data.

In order for the normalizations to be valid, the charge balance had to remain constant, so the beam energy and current were switched up and down at a very fast rate. Because of the finite slew rate of the power supplies, however, there was some lag as the voltages rose and fell. This lag was the better part of $1 \mathrm{msec}$ while switching between $1 \mathrm{kV}$ and the highest voltages, so for the 5,8 , and $13 \mathrm{keV}$ normalizations the timing pattern (shown in Figure 5.1b) was $2 \mathrm{~ms} \mathrm{high,} 2 \mathrm{~ms}$ low. A multistep pattern was used to normalize the 2,3 , and $4 \mathrm{keV}$ spectra, and a similar one for the $1.5,2.5$, and $3.5 \mathrm{keV}$ spectra (see Figure $5.1 \mathrm{c}$ ).

Because of the lag in drift tube and anode voltages, the Event Mode data acquisition system was used so that data obtained as the beam was in transition could be excluded without throwing away valid data. The amount of time actually spent at each energy could then be determined and used with the intensity of line $w$ (which depended upon only the He-like ion abundance) to compute relative excitation and ionization cross sections at different energies, after being normalized to the $1 \mathrm{keV}$ data. A more detailed explanation of this process is given in the chapter on analysis, Chapter 8. 


\section{Chapter 6}

\section{Polarization}

In order to obtain accurate line intensities, analysis of the FCS data must include such factors as window absorption and varying beam current. Another important effect is line polarization. Because of the directionality of the electron beam, X-ray emission from EBIT is iil general polarized and nonisotropic, and since the efficiency of crystal spectrometers depends upon the polarization of incident radiation, any quantitative study of EBIT spectra must take this into account. This chapter begins with a discussion of the theory of X-ray polarization, then explains in section 6.2 how theoretical polarizations were calculated for He-like neon $K_{\alpha}$ lines, continues with experimental measurement of the polarization of those lines in 6.3 , and concludes with the derivation of correction factors used to analyze low and high energy cross section data in 6.4 .

\subsection{Background and Theory}

In general, radiation produced as the result of an electron-ion collision is emitted with a specific polarization and with a continuous but nonisotropic intensity distribution $W(\theta, \phi)$, where the angles $\theta$ and $\phi$ are measured with respect to the collision axis. In most plasmas, electron-ion collisions are completely random in direction, and so radiation emitted from the plasma as a whole is both isotropic and unpolarized. In EBIT, however, the electrons all travel in one direction, introducing a preferred axis and therefore producing polarized and nonisotropic emission. Two issues thus arise which must be addressed: first, $\mathrm{X}$-rays are only observed at one angle 
relative to the beam, and second, the FCS acts as a polarimeter, preferentially reflecting $X$-rays polarized perpendicular to the plane of dispersion.

The general issue of the angular distribution and polarization of gamma rays from excited nuclei has been examined by Alder and Steffen [2]. David Vogel [80] then applied those results to the specific case of X-ray emission from EBIT as part of his $\mathrm{Ph}$. $\mathrm{D}$. thesis. The following discussion is a summary of that work, with some additions from Inal and Dubau [43].

Several simplifications of the general case can be made. First, the electron and ion distributions in EBIT are cylindrically symmetric, so the intensity distribution $W$ is only a function of $\theta$. In addition, atomic transitions are usually dominated by a single multipole operator (such as electric dipole) because the ratio of successive electric multipoles is roughly $\left(\alpha Z_{\text {eff }}\right)^{2}$, where $Z_{\text {eff }}$ is the effective nuclear charge and $\alpha$ is the fine structure coefficient. For He-like neon, the ratio is less than 1/200; magnetic multipole transition probabilities are smaller than their electric multipole counterparts by the same factor. Another simplification is that atomic X-rays have only linear and not circular polarization.

Polarization and nonistropic emission arise when the magnetic sublevels of the atomic state undergoing emission have unequal populations. The sublevels are labeled with quantum number $m_{J}$, which denotes the component of the total angular momentum $J$ which lies along the $\mathrm{z}$-axis, defined here as the electron beam direction. The occupation probability of each magnetic quantum state is given by $g\left(m_{J}\right)$ where $\Sigma g\left(m_{J}\right)=1$, summing over all $m_{J}$ values from $-\mathrm{J}$ to J. The occupation probabilities depend upon the excitation mechanism (EIE, DR, RR, cascades, etc.), but for states populated only by direct EIE,

$$
g\left(m_{j}\right)=\frac{\sigma_{m_{J}}}{\sum_{m_{J}} \sigma_{m_{J}}}=\frac{\sigma_{m_{J}}}{\sigma_{\text {total }}},
$$


where $\sigma_{m J}$ is simply the EIE cross section for each magnetic sublevel (e.g., $1 s 2 p{ }^{3} P_{2}$ with $m_{J}=-1$ ) and $\sigma_{\text {total }}$ is the cross section for that entire state (in this example, $1 s 2 p$ ${ }^{3} P_{2}$ ). Because atomic interactions are dominated by electrostatic rather than magnetic forces, occupation probabilities do not depend on the sign of $m_{J}$, that is, $g\left(m_{J}\right)=$ $g\left(-m_{J}\right)[43]$. Atomic radiation therefore has linear polarization and not circular, as would be true for the more general case where $g\left(m_{J}\right) \neq g\left(-m_{J}\right)$.

Since X-rays from EBIT are linearly polarized, their intensity can be written as the sum of two intensity distribution functions, $W_{x^{\prime}}(\theta)$ and $W_{y^{\prime}}(\theta)$, one for each polarization direction. The polarization of an X-ray emitted at an angle $\theta$ can then be defined as

$$
P(\theta)=\frac{W_{x^{\prime}}(\theta)-W_{y^{\prime}}(\theta)}{W_{x^{\prime}}(\theta)+W_{y^{\prime}}(\theta)}
$$

The emission geometry, with its axis conventions, is shown in Figure 6.1. The total intensity distribution $W(\theta)$ is simply the sum of the two components,

$$
W(\theta)=W_{x^{\prime}}(\theta)+W_{y^{\prime}}(\theta)
$$

normalized so that

$$
\int W(\theta) d \Omega=1
$$

where the integral is over all angles.

As shown by Alder and Steffen, the solutions for linearly polarized radiation from a cylindrically symmetric source (as for X-rays from EBIT) are

$$
\begin{aligned}
& W_{x^{\prime}}(\theta)=\frac{1}{8 \pi} \sum_{\lambda=\text { even }} B_{\lambda}\left(J_{i}\right)\left[A_{\lambda}(\gamma) P_{\lambda}(\cos \theta)+A_{\lambda 2} P_{\lambda}^{2}(\cos \theta)\right] \\
& W_{y^{\prime}}(\theta)=\frac{1}{8 \pi} \sum_{\lambda=\text { even }} B_{\lambda}\left(J_{i}\right)\left[A_{\lambda}(\gamma) P_{\lambda}(\cos \theta)-A_{\lambda 2} P_{\lambda}^{2}(\cos \theta)\right]
\end{aligned}
$$

where $P_{\lambda}(\cos \theta)$ and $P^{2} \lambda(\cos \theta)$ are, respectively, standard and associated Legendre polynomials. Only even terms are required for linearly polarized radiation. 


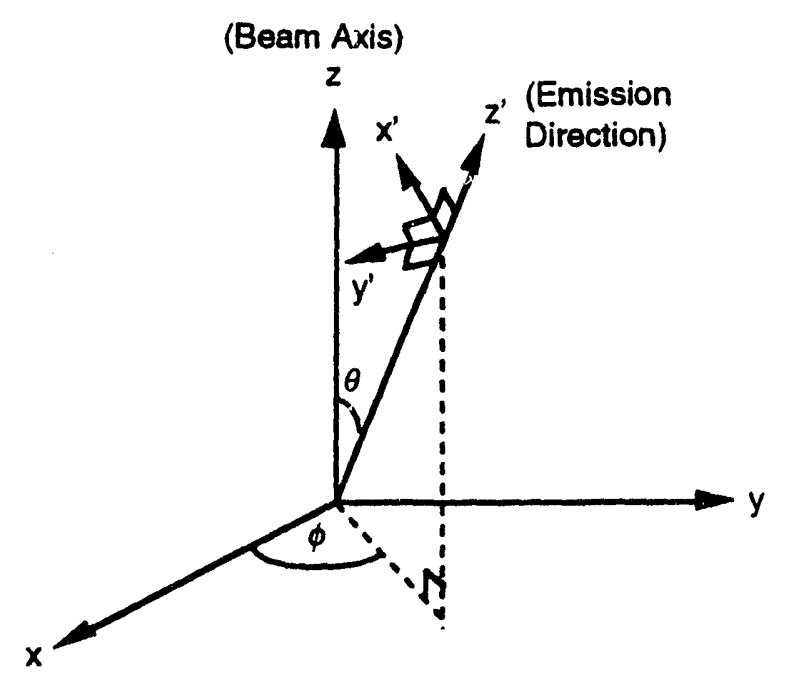

Figure 6.1: Emission geometry. $\mathrm{X}$-rays are emitted in the direction of the $z^{\prime}$ axis defined by angles $\theta$ and $\phi$. Total intensity is the sum of polarization components $W_{\mathbf{x}^{\prime}}$ and $W_{\mathbf{y}^{\prime}}$, with electric field along the $\mathrm{x}^{\prime}$ and $y^{\prime}$ axes, respectively. The $x^{\prime}$ axis lies in the plane defined by the $z$ and $z^{\prime}$ axes. When $\theta=90^{\circ}, x^{\prime}$ is parallel to the beam ( $z$ axis) and $y^{\prime}$ is perpendicular to it, so $I_{\|}$and $I_{\perp}$ are defined as being equal to $W_{\mathrm{x}^{\prime}}$ and $W_{\mathbf{y}^{\prime}}$, respectively. 
The initial state of the system is described by the function $B \lambda$, called the orientation parameter. It is a function of the initial angular momentum $J_{i}$, the magnetic quantum numbers $m_{J}$, and their populations $g\left(m_{J}\right)$ and involves Wigner 3-J symbols, which are similar to Clebsch-Gordon coefficients.

The electromagnetic transition is characterized by the angular distribution coefficient $A_{\lambda}$ and the linear polarization parameter $A_{\lambda 2}$. They are functions of both the initial and final angular momenta, and include terms proportional to the matrix elements of electromagnetic multipole operators, as well as Wigner 3-J and 6-J symbols. Dipole radiation is described by $A$ terms with $\lambda$ equal to 0 and 2 , quadrupole radiation by $\lambda=0,2$, and 4 , and so on. Note that the polarization dependence arises from the different signs of the last term in the expressions for $W_{x^{\prime}}(\theta)$ and $W_{y^{\prime}}(\theta)$. When they are added to give the total intensity distribution $W(\theta)$, the polarization dependence drops out, leaving just the directional dependence.

A thorough mathematical treatment is beyond the scope of this thesis, so only directly applicable results are presented here, beginning with some general rules. $A_{0}$ and $B_{0}$ are always equal to 1 and correspond to isotropic unpolarized radiation. States with initial total angular momentum $J$ equal to 0 or $1 / 2$ always produce radiation that is isotropic and unpolarized, regardless of the excitation mechanism Line $r$, for example, which results from the decay of the $1 s 2 s\left({ }^{3} s\right) 2 p^{2} p_{1 / 2}$ state, is always unpolarized. For cascades (discussed in section 6.2.1), the magnetic sublevel populations always become more uniform (less polarized) as one state decays to another. Another rule is that electric and magnetic multipoles of the same order produce radiation with the same intensity distribution except that the polarization components are reversed.

If we define $P$ to be the polarization at $\theta=90^{\circ}$, which is the angle at which spectra are obtained on EBIT, then for dipole radiation, 


$$
P=\frac{ \pm 3 A_{2} B_{2}}{2-A_{2} B_{2}}
$$

where the minus sign is for electric dipole (E1) and the plus sign is for magnetic dipole (M1). For magnetic quadrupole radiation, the equation is

$$
P=\frac{-3 A_{2} B_{2}-\frac{5}{4} A_{4} B_{4}}{2-A_{2} B_{2}+\frac{3}{4} A_{4} B_{4}}
$$

Making use of the equality of $g\left(m_{J}\right)$ and $g\left(-m_{J}\right)$, the orientation parameters and angular distribution coefficients for He-like $K_{\alpha}$ emission are as follows. For the dipole lines $w, y$, and $z$ (which are all $J=1$ to 0 transitions),

$$
A_{2}=\frac{1}{\sqrt{2}} \quad B_{2}=\sqrt{2}[g(1)-g(0)]
$$

while for line $q$ which has $J_{\mathrm{i}}=3 / 2$ and $J_{\mathrm{f}}=1 / 2$,

$$
A_{2}=\frac{1}{2} \quad B_{2}=2\left[g\left(\frac{3}{2}\right)-g\left(\frac{1}{2}\right)\right]
$$

Line $r$ is always unpolarized since $J_{\mathrm{i}}=1 / 2$. For line $x$, a magnetic quadrupole transition from $J=2$ to 0 , we need even terms through $\lambda=4$ :

$$
\begin{array}{ll}
A_{2}=-\sqrt{\frac{5}{14}} & B_{2}=\sqrt{\frac{10}{7}}[2 g(2)-g(1)-g(0)] \\
A_{4}=-\frac{4}{\sqrt{14}} & B_{4}=\frac{2}{\sqrt{14}}[g(2)-4 g(1)+3 g(0)]
\end{array}
$$

Using these expressions, the polarization at $90^{\circ}$ for the electric dipole emission of lines $w\left(1 s 2 p^{1} P_{1} \rightarrow 1 s^{2} S_{0}\right)$ and $y\left(1 s 2 p^{3} P_{1} \rightarrow 1 s^{2}{ }^{1} S_{0}\right)$ is given by

$$
P=\frac{g(1)-g(0)}{g(1)+g(0)}
$$

the polarization for the M1 emission of line $z\left(1 s 2 s^{3} S_{1} \rightarrow 1 s^{2}{ }^{1} S_{0}\right)$ is 


$$
P=\frac{g(0)-g(1)}{g(0)+g(1)}
$$

the polarization for the $\mathrm{E} 1$ emission of line $q\left(1 s 2 s 2 p^{2} P_{3 / 2} \rightarrow 1 s^{2} 2 s^{2} s_{1 / 2}\right)$ is

$$
P=\frac{3 g(1 / 2)-3 g(3 / 2)}{5 g(1 / 2)+3 g(3 / 2)}
$$

and the polarization for the M2 emission of line $x\left(1 s 2 p^{3} P_{2} \rightarrow 1 s^{2}{ }^{1} S_{0}\right)$ is

$$
P=\frac{g(2)-g(1)}{g(2)+g(1)}
$$

The intensity distribution functions are also required. For dipole emission (again, taking $P$ to be the polarization at $90^{\circ}$ ), the normalized intensity distribution is

$$
W(\theta)=\frac{3\left(1 \pm P \cos ^{2} \theta\right)}{4 \pi(3 \pm P)}
$$

where the minuses are for $\mathrm{El}$ and the plusses for $\mathrm{M} 1$. This provides the equation relating line intensity observed at $90^{\circ}$ with the intensity averaged over all $4 \pi$ steradians,

$$
I^{4 \pi}=\frac{3 \pm P}{3} I\left(90^{\circ}\right)
$$

There is no such simple general expression for $\mathrm{M} 2$ radiation, but for the specific case of line $x$,

$$
I^{4 \pi}=\frac{4}{5[1-g(0)]} I\left(90^{\circ}\right)
$$

\subsection{Theoretical Calculation of Line Polarizations}

All that remains is the determination of the magnetic sublevel populations $g\left(m_{J}\right)$ of each excited state. This step relies on theoretical modeling of the atomic system. 


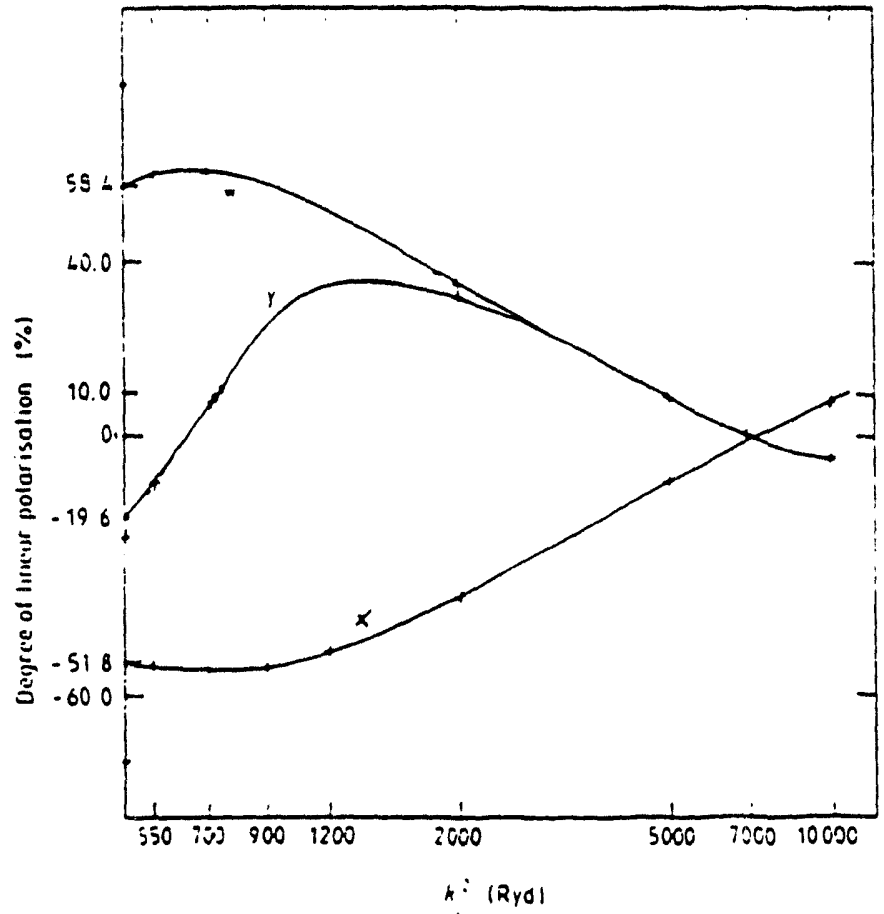

Figure 6.2: Theoretical polarization of He-like $\mathrm{Fe} K_{\alpha}$ lines arising from direct electron impact excitation [44]. Line $z$ has zero polarization when excited only by EIE. Note that the energy scale is in units of Rydbergs. Threshold is approximately $495 \mathrm{Ryd}$. 
The polarization of $K_{\alpha}$ lines excited by a unidirectional electron beam and observed at an angle of $90^{\circ}$ to the beam has been computed (neglecting cascades) by Inal and Dubau [44] for He-like Fe 24+. The results for $w, x$, and $y$ are shown in Figure 6.2. For $E I E$, and in the absence of cascades into $2^{3} S_{1}$, line $z$ is unpolarized. The polarization of $q$ was also computed and is roughly 0.55 times that of $w$ at all energies, being well approximated by the relation $P_{q}=3 P_{w} /\left(6-P_{w}\right)$.

The polarizations of He-like $\mathrm{Ne}$ were calculated using magnetic sublevel excitation cross sections provided by Reed [72]. After scaling relative to threshold energies, the results are virtually identical to those for iron, with the exception that $y$ now has the same polarization as $x$, beginning with $P=-0.52$ at threshold. The polarization of lines $x$ and $y$ diverges for higher $Z$ elements such as Fe because of the relativistic mixing of $2^{3} P_{1}$ and $2^{1} P_{1}$, causing $y$ to behave more like $w$. For He-like neon, however, the intercombination lines $x$ and $y$ have virtually identical polarization and intensity distributions, and so for purposes of analysis the $x y$ blend can be treated as one line.

\subsubsection{Cascades}

In addition to being unpolarized when directly excited by EIE, line $z$ is also unpolarized when excited by ISI of Li-like ions. This is because the electron-ion collision results in ionization, removing the affected ionic electron and leaving the remaining two electrons with no "knowledge" of the collision direction. Because $2{ }^{3} S_{1}$ is the lowest lying of any excited level, however, it receives much of its population via cascades from higher levels, especially from $2^{3} P_{2}$ and $2^{1} P_{0}$, and as a result line $z$ is expected to be somewhat polarized. $P_{z}$ can be calculated using Equation 6.13 by tracing every level that cascades into $2^{3} S_{1}$ and tracking the population of the $m_{J}=-1$, 
0 , and 1 sublevels. The bookkeeping for this analysis is most easily done by employing de-orientation factors which couple the orientation parameter of an initial state to that of the final state. Using $i$ to denote an initial state and $f$ the final, the equation is

$$
B_{\lambda}\left(J_{f}\right)=B_{\lambda}\left(J_{i}\right) U_{\lambda}\left(J_{i}, J_{f}, L\right)
$$

where $L$ is the order of the radiative multipole (all relevant cascades proceed via electric dipole transitions, so $L=1$ ) and the de-orientation factor $U_{\lambda}$ is given by

$$
U_{\lambda}\left(J_{i}, J_{f}, L\right)=(-1)^{J_{i}+J_{f}+\lambda+L}\left[\left(2 J_{i}+1\right)\left(2 J_{f}+1\right)\right]^{1 / 2}\left\{\begin{array}{lll}
J_{i} & J_{i} & \lambda \\
J_{f} & J_{f} & L
\end{array}\right\}
$$

where the \{\} term is an aforementioned Wigner 6-J symbol whose value can be found in the tables of Cowan [26]. $U_{\lambda}$ is always less than or equal to 1 , so the effect of cascading is to progressively randomize the magnetic sublevel populations and decrease the degree of polarization.

When a level is populated Ly several mechanisms or cascades from different levels, the net orientation parameter $B \lambda$ is given by weighted summing of each contribution. As an example, take the $2^{3} S_{1}$ level of He-like neon at an electron energy of $1000 \mathrm{eV}$. This is not high enough to excite the $n=3$ levels, but the $2^{3} S_{1}$ state is still fed by cascades from the $2^{3} P_{2,1,0}$ levels in addition to being directly excited by EIE. Branching ratios for He-like Ne levels through $n=3$ were computed by Osterheld [66] for this experiment and are shown in Table 6.1. The equation for the $B_{2}$ orientation parameter of the $2^{3} S_{1}$ state is then

$$
B_{2}\left({ }^{3} S_{1}\right)=\frac{\sum_{i} U_{2}\left(J_{i}, 1,1\right) \sigma_{i} B_{2}\left(J_{i}\right) b_{i \rightarrow z}}{\sum_{i} \sigma_{i} b_{i \rightarrow z}}
$$




\begin{tabular}{ll}
\hline Level & Branching ratio \& transition \\
\hline $2^{3} S_{1}$ & $1.000 \rightarrow 1^{1} S_{0}(\mathrm{z})$ \\
$2^{3} P_{0}$ & $1.000 \rightarrow 2^{3} S_{1}$ \\
$2^{3} P_{1}$ & $0.965 \rightarrow 1^{1} S_{0}(\mathrm{y})$ \\
& $0.035 \rightarrow 2^{3} S_{1}$ \\
$2^{3} P_{2}$ & $0.020 \rightarrow 1^{1} S_{0}(\mathrm{x})$ \\
& $0.980 \rightarrow 2^{3} S_{1}$ \\
$2^{1} S_{0}$ & $1.000 \rightarrow 1^{1} S_{0}(2$-photon $)$ \\
$2^{1} P_{1}$ & $1.000 \rightarrow 1^{1} S_{0}(\mathrm{w})$ \\
$3^{3} S_{1}$ & $0.67 \rightarrow 2^{3} P_{0} \rightarrow 2^{3} S_{1}$ \\
& $0.33 \rightarrow 2^{3} P_{1}$ \\
$3^{3} P_{0}$ & $1.00 \rightarrow 2^{3} S_{1}$ \\
$3^{3} P_{1}$ & $0.99 \rightarrow 2^{3} S_{1}$ \\
$3^{3} P_{2}$ & $1.00 \rightarrow 2^{3} S_{1}$ \\
$3^{1} S_{0}$ & $1.00 \rightarrow 2^{1} P_{1}$ \\
$3^{3} D_{1}$ & $0.56 \rightarrow 2^{3} P_{0} \rightarrow 2^{3} S_{1}$ \\
& $0.42 \rightarrow 2^{3} P_{1}$ \\
$3^{3} D_{2}$ & $0.74 \rightarrow 2^{3} P_{1}$ \\
& $0.24 \rightarrow 2^{3} P_{2} \rightarrow 2^{3} S_{1}$ \\
$3^{3} D_{3}$ & $0.98 \rightarrow 2^{3} P_{2} \rightarrow 2^{3} S_{1}$ \\
$3^{1} D_{2}$ & $0.98 \rightarrow 2^{1} P_{1}$ \\
$3^{1} P_{1}$ & $1.00 \rightarrow 1^{1} S_{0}$ \\
\hline \hline
\end{tabular}

Table 6.1: List of branching ratios for levels with $n=2$ and $n=3$, calculated by Osterheld [66]. For higher- $n$ levels, it is assumed that branching ratios for each term are the same as for its $n=3$ counterpart. Extrapolation of EIE cross sections assumes that $\sigma$ scales as $n^{-4.5}$ for triplet terms and $n^{-3.6}$ for singlets. Excitation to $F, G$, and higher terms is negligible. 


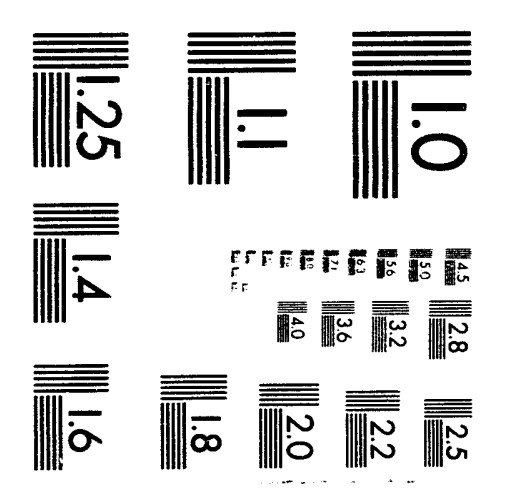



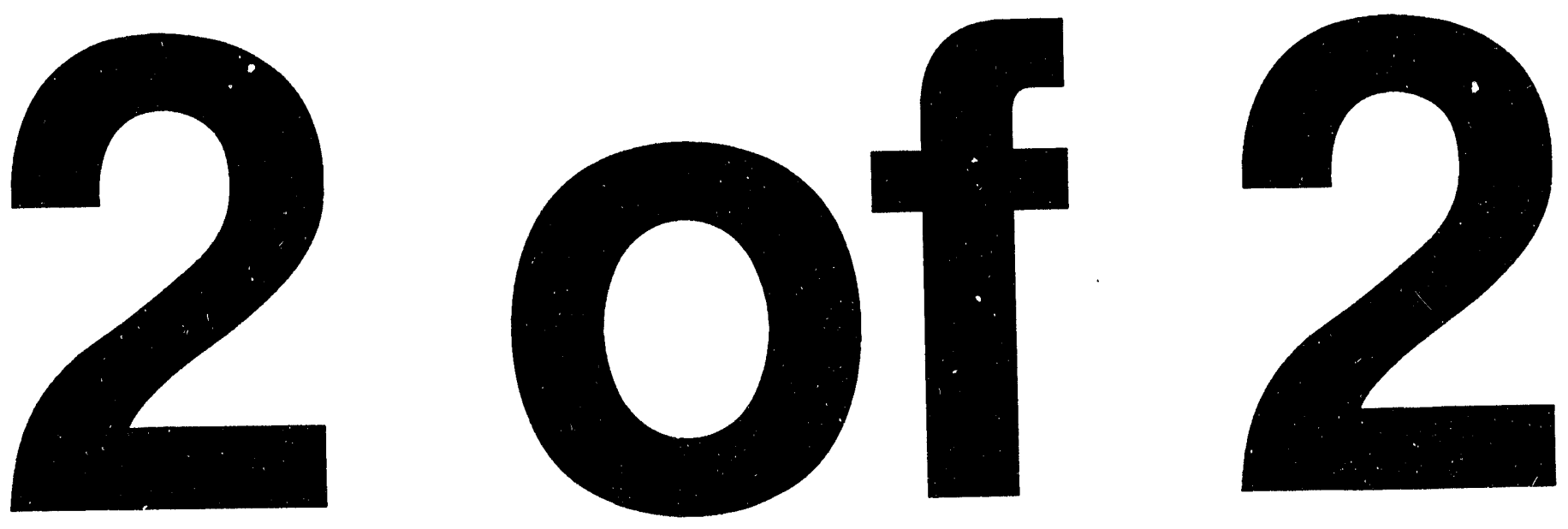
where $\sigma_{i}$ is the excitation cross section for an initial level and $b_{i \rightarrow z}$ is the branching ratio for radiative decay of that level into $2^{3} S_{1}$. The denominator is the total effective excitation cross section for $2^{3} S_{1}$. Continuing with this example, the de-orientation factors for the $2^{3} P$ levels are

$$
\begin{array}{ll}
\text { 1s } 2 \text { p }{ }^{3} P_{2}: & U_{2}(2,1,1)=(7 / 20)^{1 / 2}=0.5916 \\
1 s 2 p^{3} P_{1}: & U_{2}(1,1,1)=1 / 2 .
\end{array}
$$

Level ${ }^{3} P_{0}$ is already unoriented $\left(J_{i}=0\right)$ so its $B_{2}=0$. The direct EIE contribution of $2^{3} S_{1}$ is also unoriented. Substituting in the expressions for $B_{2}$ for each level along with the branching ratios, the numerator of equation 6.18 is then

$$
\begin{aligned}
& \sqrt{\frac{7}{20}} \sigma\left(2^{3} P_{2}\right) \sqrt{\frac{10}{7}}[2 g(2)-g(1)-g(0)] 0.980 \\
& +\frac{1}{2} \sigma\left(2^{3} P_{1}\right) \sqrt{2}[g(1)-g(0)] 0.035 \\
& +0\left(\text { for } 2^{3} P_{0}\right)+0\left(\text { for } 2^{3} S_{1}\right)
\end{aligned}
$$

and the denominator is

$$
\sigma\left(2^{3} P_{2}\right) 0.980+\sigma\left(2^{3} P_{1}\right) 0.035+\sigma\left(2^{3} P_{0}\right) 1.000+\sigma\left(2^{3} S_{1}\right) 1.000
$$

Cross sections for EIE of the magnetic sublevels of all $n=2$ and $n=3$ levels in He-like Ne were calculated by Reed for several energies between 930 and 13,000 eV [72]. For $n \geq 4$, cross sections for $S, P$, and $D$ terms were extrapolated assuming that they scale as $n^{-4.5}$ for triplet terms and $n^{-3.6}$ for singlets. Those powers of $n$ were derived from the ratios of $n=2$ and $n=3$ cross sections. Excitation to terms of higher angular momentum $(F, G$, etc.) was assumed to be negligible. Decay channels and branching ratios were assumed to be the same as for corresponding $n=3$ terms. Cross sections for innershell EIE of Li-like neon were also computed by Reed. These cross sections were then used to calculate the polarization of lines $w, x, y, z$, and $q$ at all 


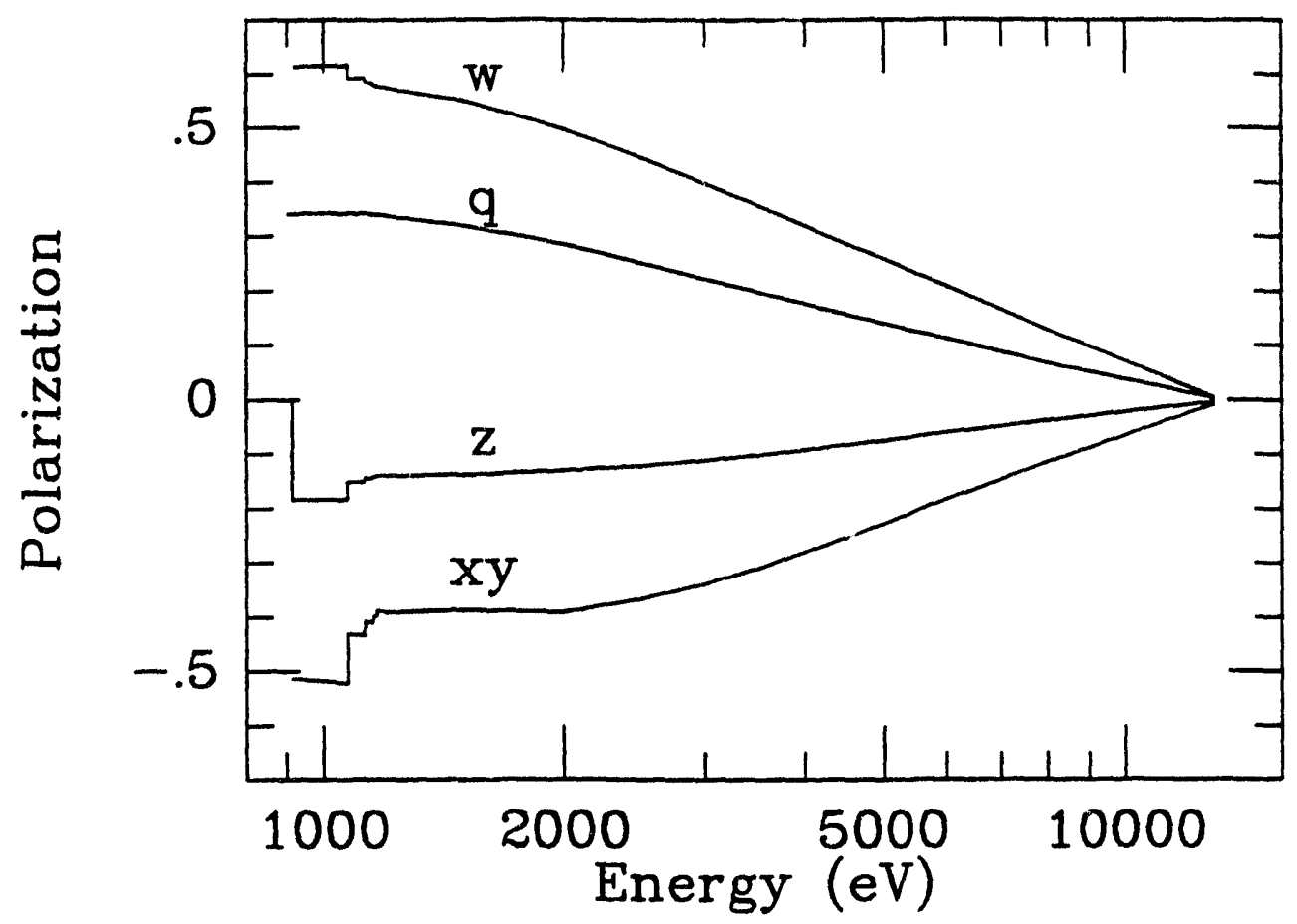

Figure 6.3: Theoretical polarizations of He-like $\mathrm{Ne} K_{\alpha}$ lines, derived from cross section calculations of Reed [72]. Compare with Figure 6.2 for He-like Fe, which does not include the effect of cascades. Line $r$, which is blended with $q$, always has zero polarization because it proceeds from a level with $J_{\mathrm{i}}=1 / 2$. 
energies between threshold and $1200 \mathrm{eV}$, as well as at the higher energies for which spectra were obtained Resuits are shown in Figure 6.3. The effects of resonant excitation peaks were not included since no theoretical calculations of their polarization exist. It was implicitly assumed that RE emission has the same polarization as EIE emission.

\subsubsection{Collisional Transfer from the Metastable ${ }^{3} S_{1}$ State}

The above results are only valid in the low-density limit. At densities typically found in EBIT, the $2^{3} S_{1}$ level is sometimes excited to higher levels before it can decay to ground and emit a $z$ photon. Collisional transfer not only increases the intensity of $x y$ at the expense of $z$, it can also affect those lines' polarizations. I used the same model to study this effect as I did for cascades, with the addition of possible excitation from the $2^{3} S_{1}$ level rather than just decay to ground. The branching ratio for excitation to a state $s_{k}$ from the $2^{3} S_{1}$ level depends on the electron density and energy and is given by

$$
b_{k}=\frac{n_{e} v_{e} \sigma\left(s_{k}\right)}{n_{e} v_{e} \sum_{j} \sigma\left(s_{j}\right)+A_{z}}
$$

where $\sigma\left(s_{k}\right)$ is the cross section for collisional transfer to state $s_{k}$, the sum is over all states that can be excited from $2^{3} S_{1}$, and $A_{z}$ is the radiative rate for line $z$, which was measured in Chapter 7. Note, however, that because we are dealing with magnetic sublevels, the radiative rate used in equation 6.24 is actually one-third the total radiative rate of the ${ }^{3} S_{1}$ level, since $m_{J}$ can equal $-1,0$, or +1 .

According to calculations by Osterheld [66], 89\% of collisional transfers from $2^{3} S_{1}$ go directly to $2^{3} P_{2,1,0}$ and the other $11 \%$ to higher energy states (mostly $n=3$ ) 
that then decay back to $2^{3} S_{1}$ or $2^{3} P_{2,1,0}$. My model used only the cross sections for direct transfer to the $2^{3} P$ states, but approximated the effect of transfer to intermediate states by replacing $\sigma\left(s_{k}\right)$ with $1.07 \sigma\left(s_{k}\right)$ in equation 6.24 . Cross sections for excitation to the magnetic sublevels of $2^{3} P_{2,1,0}$ at an energy of $750 \mathrm{eV}$ were provided by Sampson and Zhang [74]. They also provided cross sections at energies of 1200 and $2000 \mathrm{eV}$, but only for the magnetic sublevels of ${ }^{3} P_{1}$. Their results showed that cross sections at each energy (for each $m_{\mathrm{J}}$ ) were virtually the same after scaling the cross sections as $E^{-0.84}$. Sublevel cross sections for ${ }^{3} P_{2}$ and ${ }^{3} P_{0}$ were assumed to scale the same way.

To determine the appropriate value of $n_{\mathrm{e}}$ to use in equation 6.24 . I conducted an experiment, discussed in detail in Chapter 7, that measured the net transfer of $z$ to $x y$ intensity at $750 \mathrm{eV}$ while also measuring the radiative rate of $z$. At a beam current of $42 \mathrm{~mA}$, the ratio of transfer-produced $I_{x y}$ to $I_{z}$ was 0.218 . According to my model, that required a density of $5.7 \times 10^{11} \mathrm{~cm}^{-3}$. (Density results listed in Chapter 7 were derived using a separate model constructed by Osterheld that did not break down cross sections by magnetic sublevel. That model predicted a density of $8.7 \times 10^{11} \mathrm{~cm}^{-3}$, a factor of 1.5 higher, which is consistent with its use of cross sections for collisional transfer that were $2 / 3$ smaller than those of Zhang and Sampson. This points out the uncertainty inherent in calculating cross sections for this process, but has no effect on calculated polarizations since cross sections predicted by the two theories differ by only a scale factor. In that case, $n_{\mathrm{e}}$ can be chosen so that the product $n_{\mathrm{e}} \sigma$ is the same for both theories.) The density obtained using this method is significantly lower than the $4.2 \times 10^{12} \mathrm{~cm}^{-3}$ predicted using equation 3.2 which is based on the diameter of the beam. The reason is that ions move in and out of the electron beam, and since the ${ }^{3} S_{1}$ state is so long-lived, it effectively "sees" a lower average density. This effect will be discussed in more detail in the results section of Chapter 8. 
Using the density determined above, the collisional transfer model showed that there was no effect on the polarization of $z$ at any energy, while the polarization of $x y$ emission as a result of collisional transfer was always equal to $P_{z}+0.177$. This positively polarized emission component thus makes the net polarization of $x y$ less negative than in the low-density limit (seen in Figure 6.3). As an example, at $1 \mathrm{keV}$, $P_{x y}$ was reduced from -0.519 (its low-density limit) to -0.325 . At $13 \mathrm{keV}, P_{x y}$ even becomes slightly positive.

At energies below the EIE threshold, DR satellite lines are also polarized, with the polarizations of high- $n$ satellites smoothly approaching those of their parent lines as $n \rightarrow \infty$. The polarizations of $K L L$ DR lines are the same for all elements and have been calculated by Inal and Dubau [43]. DR satellites are discussed in detail in Chapter 8.

\subsubsection{Effect of Transverse Electron Velocity}

The theoretical predictions described above must be adjusted because electron collisions in EBIT are not entirely unidirectional. There is a transverse velocity component arising from the rotation of the entire beam at one half the cyclotron frequency as it travels upward along the magnetic field [41]. With a magnetic field of 3 Tesla, this produces a transverse temperature of roughly $250 \mathrm{eV}$, tipping the direction of each electron-ion collision from purely vertical. The resulting distribution of collision directions remains centered on the electron beam axis, but with a relative velocity spread $\Delta v_{\mathrm{e}} / v_{\mathrm{e}}$ that is proportional to the inverse square root of the longitudinal beam energy. The net effect is to make collisions more isotropic and reduce the polarization of $\mathrm{X}$-rays emitted from the trap. In their study of He-like Sc polarization

on EBIT [39], Henderson et al. calculated that the actual line polarization (from purely 
unidirectional collisions) was approximately $10 \%$ greater (e.g., $P=0.44$ versus 0.40 ) than the polarization of lines emitted from the trap. Their measurement was at a beam energy of $4.4 \mathrm{keV}$. Extrapolating to other energies, the effect at $1 \mathrm{keV}$ is estimated to be larger by a factor of $(4.4 / 1.0)^{1 / 2}$, so that the correction is $21 \%$, and the equation relating "unidirectional" polarization and the emitted polarization of EBIT X-rays at any energy is given by

$$
P_{\text {emisted }}(E)=\left(1-\frac{0.21}{\sqrt{E(k e v)}}\right) P_{u n i}(E)
$$

Applying this to line $w$ at a beam energy of $1.0 \mathrm{keV}$, the expected polarization will be 0.79 times +0.615 , or +0.486 . Likewise, lines $x y$ and $z$ should have polarizations of -0.258 and -0.145 , respectively. One final step is to smooth the sharp jumps in polarization that occur at cascade thresholds in order to duplicate the effect of the spread in beam energy arising from space charge. This was done by convolving polarization as a function of electron energy with a Gaussian function $(F W M H=50$ $\mathrm{eV}$, or $\sqrt{2} \sigma=30.0 \mathrm{eV}$ ), weighted by the effective cross section and the nonisotropic emission factor (from equation 6.17), that is,

$$
P_{\text {smooth }}(E)=\frac{\int_{-\infty}^{\infty} P\left(E^{\prime}\right) \sigma\left(E^{\prime}\right) \frac{3}{3 \pm P(E)} e^{-\left(\frac{E-E}{30.0}\right)^{2}} d E^{\prime}}{\int_{-\infty}^{\infty} \sigma\left(E^{\prime}\right) \frac{3}{3 \pm P\left(E^{\prime}\right)} e^{-\left(\frac{E-E}{30.0}\right)^{2}} d E^{\prime}} .
$$

The " $+"$ in the intensity distribution factor is for $z$, a magnetic dipole transition, while "-" is for the other lines. As mentioned before, cross sections were obtained from the same model used to calculate line polarizations. The integrals were numerically evaluated by summing between $E-50$ and $E+50 \mathrm{eV}$ in $1-\mathrm{eV}$ steps. Final results are shown in Figure 6.4. For comparison with experimental cross section data (which is 


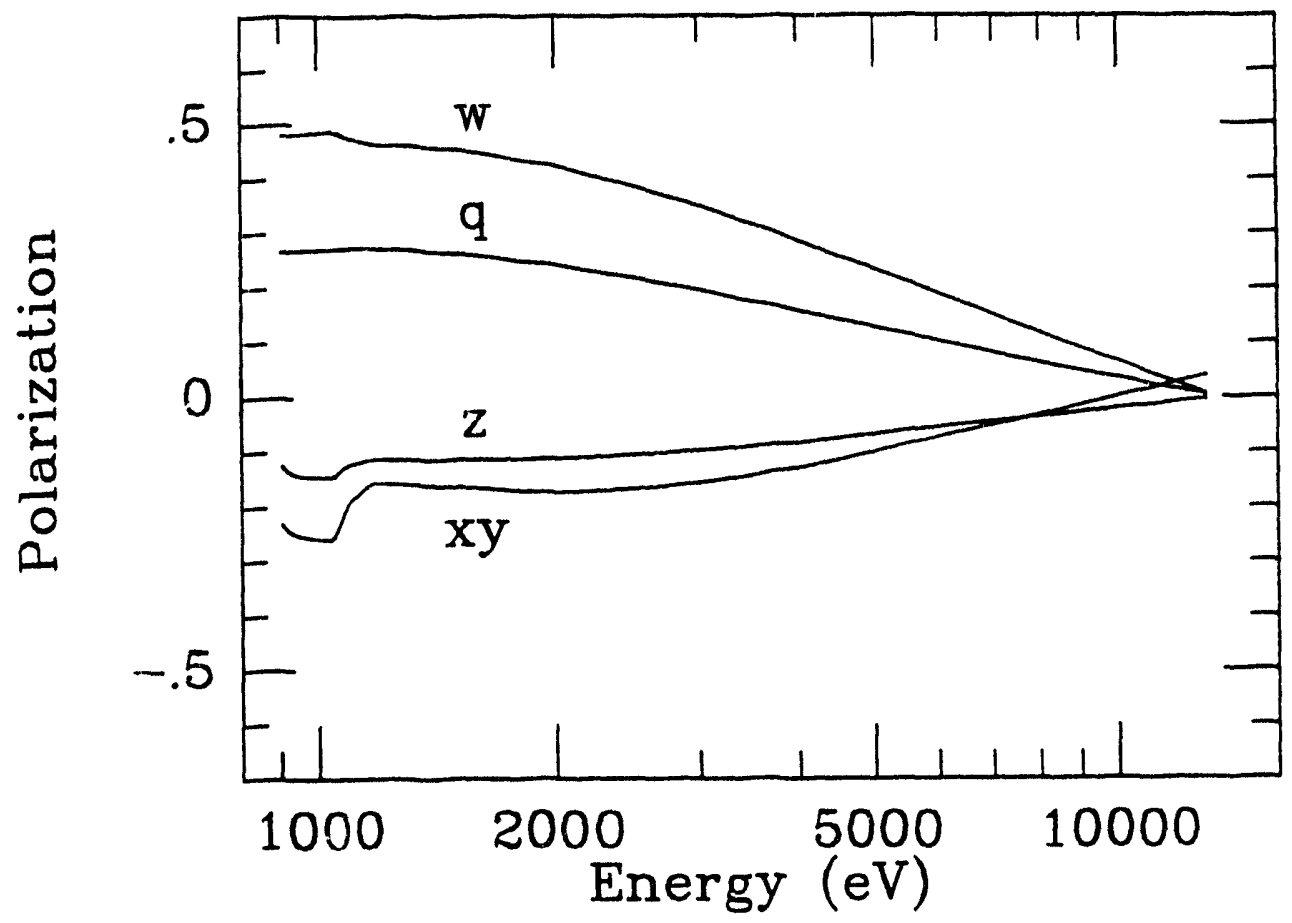

Figure 6.4: Theoretical polarizations after including the effects of collisional transfer, transverse electron velocity, and beam energy spread. These results are only for direct excitation of He-like ions. The polarization of line $z$ (and $x y$ via collisional transfer) will be altered when excited as a result of innershell ionization of Li-like ions. 
presented in Chapter 8), theoretical cross sections must also be convolved with the beam energy distribution, using the equation

$$
\sigma_{\text {smooth }}(E)=\frac{\int_{-\infty}^{\infty} \sigma\left(E^{\prime}\right) e^{-\left(\frac{E-E}{30.0}\right)^{2}} d E^{\prime}}{\int_{-\infty}^{\infty} e^{-\left(\frac{E-E}{30.0}\right)^{2}} d E^{\prime}} .
$$

To summarize this rather lengthy section, effective cross sections and polarizations of He-like neon $K_{\alpha}$ lines were derived from theoretical cross sections for excitation of magnetic sublevels in $n=2$ and $n=3$ states by a perfectly collimated monoenergetic electron beam. The effects of cascades from all levels, as well as collisional transfer from the $2^{3} S_{1}$ metastable state, were included. In order to apply those results to spectra obtained using EBIT, the effects of transverse electron velocity and energy spread in the EBIT electron beam were also computed. It was found that cascades have a very significant effect on the polarizations of $x y$ and $z$, and that collisional transfer from $z$ to $x y$ reduced the degree of polarization of $x y$ while having no effect on $P_{z}$. Transverse electron velocities in the EBIT beam tend to make ionelectron collisions more omni-directional, which reduces the polarization of all lines.

\subsection{Polarization Measurements}

Although theoretical predictions of He-like line polarization are more reliable than cross section calculations (since it is generally only the relative cross sections that matter) and should be accurate to several percent, it is still desirable to have experimental confirmation. In one of the few X-ray measurements that has been made ( $K_{\alpha}$ emission from He-like Sc ${ }^{19+}$ [39]), measured polarizations differed sharply with 
theory until the hyperfine interaction was included in theoretical models [39]. (None of the isotopes of neon has any spin. so there is no hyperfine coupling.)

\subsubsection{Experimental Methods}

There are three methods of measuring polarization on EBIT, all of them rather difficult, each having its own strengths and weaknesses. The first is to record spectra at different values of the angle $\theta$, making measurements on one of the ports ringing the $\operatorname{trap}\left(\theta=90^{\circ}\right)$ and at the top of EBIT $\left(\theta=0^{\circ}\right)$. Polarization can then be determined (after normalizing for the different observation geometries, usually by using an unpolarized line for comparison) based on the relationship between $P$ and the intensity distribution function $W(\theta)$, using equation (6.16) or a similar equation appropriate for the transition involved. Because of the very small opening angle for observations at $0^{\circ}$, however, measurements using a crystal spectrometer are far too time-consuming and solid state detectors must be used. Because of those detectors' limited energy resolution, most emission lines can not be observed this way.

The second and third methods both rely on the fact that diffraction crystals have different reflectivities for radiation polarized perpendicular and parallel to the plane of dispersion. Since virtually all measurements on EBIT are made at $\theta=90^{\circ}$, let us again take $P$ to stand for $P\left(90^{\circ}\right)$ and define the quantities

$$
\begin{aligned}
& I_{\|}=W_{x^{\prime}}\left(90^{\circ}\right) \\
& I_{\perp}=W_{y^{\prime}}\left(90^{\circ}\right)
\end{aligned}
$$

so that $I_{\|}$refers to intensity with polarization parallel to the electron beam (perpendicular to the plane of dispersion) and $I_{\perp}$ refers to intensity with polarization 
perpendicular to the beam (parallel to the plane of dispersion). Let $R_{\|}$and $R_{\perp}$ refer to the corresponding crystal reflectivities. The polarization is then given by

$$
P=\frac{I_{\|}-I_{\perp}}{I_{\|}+I_{\perp}}=\frac{\left(I_{\|} / I_{\perp}\right)-1}{\left(I_{\|} / I_{\perp}\right)+1}
$$

and the total measured intensity (at $\theta=90^{\circ}$ ) is given by

$$
I_{\text {meas }}=I_{\|} R_{\|}+I_{\perp} R_{\perp} .
$$

Using the "rotatable spectrometer" method, spectra are collected with a crystal spectrometer in two different orientations, the normal horizontal mode and a vertical mode. In the vertical mode, $R_{\|}$and $R_{\perp}$ are reversed in equation 6.31 . With $R_{\|}$and $R_{\perp}$ known from theory or calibration (and after normalizing the different geometrical factors using a line of known polarization), $I_{||} I_{\perp}$ can then be determined. If the crystal reflectivity is much higher for one polarization component than the other, as is true for Bragg angles near $45^{\circ}$ since $R_{\perp} / R_{\|} \approx \cos ^{2}\left(2 \theta_{\mathrm{B}}\right)$, then the analysis is particularly straightforward since only one polarization component is being measured at a time. This is the method that was used by Henderson et al. [39] to measure the polarization of $w, x, y$, and $z$ in He-like $\mathrm{Sc}^{19+}$, a measurement that revealed the importance of the hyperfine interaction in that system. Although this method is conceptually simple, determining the relative geometrical efficiency of the two spectrometer orientations can be quite time consuming even with a curved crystal. The situation would be even worse using a flat crystal spectrometer because the EBIT line source would have to be stopped down to a point source for the vertical measurement. In addition, two spectrometers must be used, or the same one moved to collect spectra in the two different orientations. For these reasons, my polarization measurements employed the third procedure in which the FCS did not have to be moved, as explained below. 


\subsubsection{Polarization at $1 \mathrm{keV}$}

The method used here was the "two crystal" technique, which employs two crystals with different values of $R_{\perp} / R_{\|}$. In this polarization measurement for He-like Ne $K_{\alpha}$ lines, TAP and mica crystals were used, with Bragg angles of approximately $32^{\circ}$ and $43^{\circ}$, respectively. The corresponding values of $R_{\perp} / R_{\|}$, which are slightly different for each line, are approximately 0.24 and 0.006 according to theoretical calculations by Gullikson, listed in Ref. [40]. The two equations for measured intensity,

$$
\begin{gathered}
I_{\text {meas }}^{T A P}=I_{\|} R_{\|}^{T A P}+I_{\perp} R_{\perp}^{T A P} \\
I_{\text {meas }}^{\text {mica }}=I_{\|} R_{\|}^{\text {mica }}+I_{\perp} R_{\perp}^{\text {mica }},
\end{gathered}
$$

can be arranged to give

$$
\frac{I_{\|}}{I_{\perp}}=\left(\frac{R_{\perp}^{\text {TAP }}}{R_{\|}^{\text {TAP }}}\right) \frac{\left(\frac{I_{\text {meas }}^{\text {mica }}}{I_{\text {meas }}^{\text {TAP }}}\right)-\left(\frac{R_{\perp}^{\text {mica }}}{R_{\perp}^{\text {TAP }}}\right)}{\left(\frac{R_{\|}^{\text {mica }}}{R_{\|}^{\text {TAP }}}\right)-\left(\frac{I_{\text {meas }}^{\text {mica }}}{I_{\text {meas }}^{\text {TAP }}}\right)} .
$$

Because the TAP and mica data are collected at different times, the measured line intensities are normalized to the $K_{\alpha}$ peak seen in the $\mathrm{Si}(\mathrm{Li})$ detector data, which are insensitive to polarization. The polarization is then given by equation (6.30).

Spectra were collected at $1000 \mathrm{eV}$, and are shown in Figure 6.5. This energy was chosen because it lies below the onset of cascades and between resonances, but is still near threshold where it is most important that cross sections be accurate. Although the TAP and mica measurements were taken at different times (first the TAP, then two sets of mica measurements, then TAP again), the number of $K_{\alpha}$ counts in the $\mathrm{Si}(\mathrm{Li})$ data provided very reliable normalizations. Mica spectra were also 

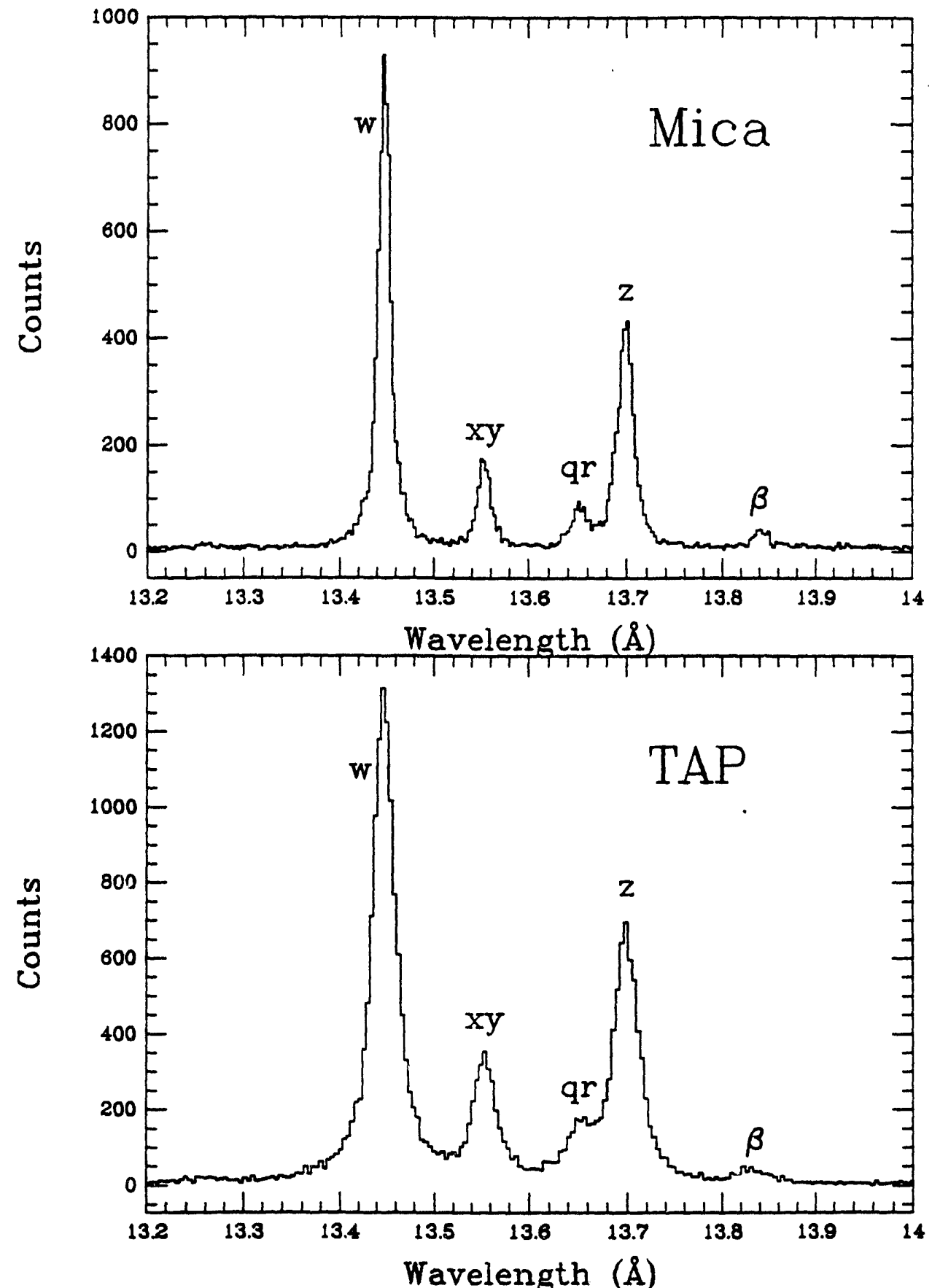

Figure 6.5: Spectra obtained at $1000 \mathrm{eV}$ with mica and TAP crystals. Note that $x y$ is relatively less intense in the mica spectrum. This is because $x y$ is negatively polarized $\left(I_{\perp}>I_{\|}\right)$and the reflectivity of mica is very low for perpendicularly polarized radiation of wavelength $\lambda_{x y}$. 
obtained in Event Mode fashion over the energy range $750-1200 \mathrm{eV}$, just as was done using a TAP crystal.

Crystal reflectivities for each line were interpolated from the tabulated results of Gullikson. Although both crystals used in this experiment were new and presumably in good condition, their uniformity was checked by looking at the intensity of line $w$, normalized to the $\mathrm{Si}(\mathrm{Li})$ detector peak, as the crystal was rotated in small increments, thus moving the active diffraction area for line $w$ across the crystal face. It was found that the TAP reflectivity varied smoothly and linearly along the crystal, producing a $6.5 \%$ higher reflectivity for line $z$ than line $w(2.7 \%$ higher for $x y, 5.3 \%$ for $q)$. A trend of the same magnitude was also seen in the mica data, although with lesser statistics because of mica's significantly lower overall reflectivity, approximately $1 / 12$ that of TAP. In equation 6.34, these two effects cancel each other out.

The theoretical values of $R_{\|} R_{\perp}$ for each crystal should be quite accurate, but the ratios $R_{\|}^{\text {mica }} / R_{\|}^{\text {TAP }}$ and $R_{\perp}^{\text {mica }} / R_{\perp}^{\text {TAP }}$ are less reliable and vary a bit with each pair of crystals. They can be determined from equation 6.34 by solving for $R_{\|}^{\text {mica }} / R_{\|}^{\text {TAP }}$ after substituting

$$
\frac{R_{\perp}^{\text {mica }}}{R_{\perp}^{\text {TAP }}}=\frac{R_{\perp}^{\text {mica }}}{R_{\|}^{\text {mica }}} \frac{R_{\|}^{\text {mica }}}{R_{\|}^{\text {TAP }}} \frac{R_{\|}^{\text {TAP }}}{R_{\perp}^{\text {TAP }}}
$$

and observing a nearby line of known polarization, usually an intrinsically unpolarized line since no theoretical calculations are then required. For $\mathrm{He}$-like $\mathrm{Ne}$, however, there are no suitable lines with $P=0$. The nearby hydrogenic Ne Lyman- $\alpha_{2}$ line is unpolarized but blended with polarized Lyman- $\alpha_{1}$, and the only unpolarized Li-like satellite with significant intensity is line $r$, which is blended with $q$. All the $K_{\alpha}$ lines are unpolarized at $13 \mathrm{keV}$, but countrates are too low at that energy to acquire the excellent statistics required. Instead, line $w$ was chosen because of its high intensity and because it is unaffected by collisional transfer. Thus, its theoretically predicted polarization should be quite reliable. 
Measured intensities for $w$ and the other lines were obtained using the PKFIT peak-fitting program written by David Knapp, an EBIT scientist. It uses standard $\chi^{2-}$ minimization methods and offers great flexibility in choosing the fitting region, type of curve to use, and links between peaks. It was found empirically that a weak Gaussian function on top of a broader Lorentzian function gave the best fits. Each peak was therefore described by six parameters, namely the amplitude, width, and centroid of each function. Widths should be equal for all the peaks, so those two parameters were automatically set to the best-fit values for line $w$, which was always the strongest line. The Gaussian and Lorentzian functions were also set to have the same centroids for a given peak, and their relative amplitudes were also linked to the value found for the $w$ peak. For lines other than $w$, therefore, only two parameters were left free to vary, an amplitude and the centroid. All lines sat on top of a common flat background which was free to vary. The fits obtained in this way were quite reliable, and the uncertainty in the number of counts for each peak was taken to be $N^{1 / 2}$. Background was completely negligible except for $z$, which was blended with a very weak line. The intensity of this background line was determined from comparison with another unblended contaminant line. (The intensities of all background lines were checked at several energies when no neon was present in the trap, and only one line, under line $z$, interfered with any of the neon lines, and only at energies below about $2 \mathrm{keV}$.) Its intensity was approximately $1 \%$ that of $z$, with a relative uncertainty of $\pm 20 \%$ which was included in the error estimate for $I_{z}$.

Over 22,000 counts were accumulated in $w$ using TAP, and over 7000 using mica. Using the $K_{\alpha}$ peak in the $\mathrm{Si}(\mathrm{Li})$ detector data for normalization, the intensity ratio for $w$ was $I_{\text {meas }}^{\text {mica }} / I_{\text {meas }}^{\text {TAP }}=0.0822 \pm 0.0005$. A polarization of +0.486 for $w$ means $I_{\|} I_{\perp}=2.89$, and inserting these values into equation 6.34 yields $R_{\|}^{\text {mica }} / R_{\|}^{\text {TAP }}=$ 
$0.0889 \pm 0.0012$. Using that value, the polarizations of the other lines were determined and are listed in Table 6.2.

Polarization data from the second measurement, collected over the range 750 $1200 \mathrm{eV}$, had to be summed to attain adequate statistics. Below about $920 \mathrm{eV}$ lie the DR satellite lines, whose strengths and polarizations are relatively more difficult to calculate theoretically. For this reason, only spectra between 920 and $1200 \mathrm{eV}$ were summed. These polarization data were analyzed in the same fashion as the $1000 \mathrm{eV}$ data, defining $P_{\mathrm{w}}$ to be +0.477 , which was computed from a weighted average of the theoretical polarizations shown in Figure 6.4. Those results are also listed in Table 6.2 .

As seen from equation 6.34, errors in the measured polarizations can arise from several sources. Theoretical values of $R_{\|} / R_{\perp}$ for both TAP and mica are fairly reliable, and have little effect on $P$ even if their values are grossly wrong. $P$ is much more sensitive to the value of $R_{\|}^{\text {mica }} / R_{\|}^{T A P}$, which was determined by setting the measured value of $P_{\mathrm{w}}$ equal to the theoretical value. The uncertainty in $R_{\|}^{\text {mica }} / R_{\|}^{\text {TAP }}$ is almost exactly equal to the uncertainty in $I_{\text {meas }}^{\text {mica }} / I_{\text {meas }}^{T A P}$ for line $w$, which (for the $1 \mathrm{keV}$ data) is equal to $1.4 \%$ from simple counting statistics. That uncertainty in the value of $R_{\|}^{\text {mica }} / R_{\|}^{T A P}$ propagates through equation 6.34 to create uncertainties in $P_{\mathrm{xy}}$ and $P_{\mathrm{Z}}$ of 0.051 and 0.044 , respectively. The statistical uncertainties in the values of $I_{\text {meas }}^{\text {mica }} / I_{\text {meas }}^{\text {TAP }}$ for $w, x y$, and $z$ likewise create uncertainties in $P$ of $\pm 0.074,0.054$, and 0.041 , respectively. Adding in quadrature yields uncertainties of $\pm 0.074,0.074$, and 0.044 for $P_{\mathrm{w}}, P_{\mathrm{xy}}$, and $P_{\mathrm{z}}$, respectively. Uncertainties for the $920-1200 \mathrm{eV}$ data are nearly identical to those for $1000 \mathrm{eV}$, given above. Uncertainties in theoretical polarizations are difficult to estimate accurately, but should be a few or several percent.

Although the TAP and mica spectra included the $q r$ blend, $P_{\mathrm{gr}}$ is not listed. This is because its value is very sensitive to $I_{\text {meas }}^{\text {mica }} / I_{\text {meas }}^{T A P}$, and since there is no way to ensure 


\begin{tabular}{|lc|ll|}
\hline \hline \multicolumn{2}{|c|}{$\begin{array}{c}\text { Polarization } \\
\text { at } 1000 \mathrm{eV}\end{array}$} & \multicolumn{2}{c|}{ Average Polarization } \\
Theoretical & Measured & Theoretical $1200 \mathrm{eV}$ \\
\hline$P_{\mathrm{w}}=+0.486$ & $P_{\mathrm{w}} \equiv+0.486$ & $P_{\mathrm{w}}=+0.477$ & $P_{\mathrm{w}} \equiv+0.477$ \\
$P_{\mathrm{xy}}=-0.258$ & $P_{\mathrm{xy}}=-0.277$ & $P_{\mathrm{xy}}=-0.217$ & $P_{\mathrm{xy}}=-0.292$ \\
$P_{\mathrm{z}}=-0.145$ & $P_{\mathrm{z}}=+0.618$ & $P_{\mathrm{z}}=-0.130$ & $P_{\mathrm{z}}=+0.765$ \\
\hline
\end{tabular}

Table 6.2: Theoretical and experimentally measured polarizations of He-like neon $K_{\alpha}$ lines. The measured polarization of line $w$ is defined to be equal to the theoretical polarization. Statistical uncertainties are \pm 0.074 for $P_{\mathrm{w}}, \pm 0.074$ for $P_{\mathrm{xy}}$, and \pm 0.044 for $P_{\mathrm{z}}$. Measured and predicted values of $P_{x y}$ show good agreement. It is believed the measured values of $P_{\mathrm{z}}$ are incorrect because of defects in the mica diffraction crystal. 
that the ratio of He-like to Li-like ions was the same for the TAP and mica data at the percent level, the measured value of $P_{\mathrm{qr}}$ cannot be trusted. A small variation in $n_{\mathrm{He}} / n_{\mathrm{Li}}$ has negligible effect on the measured values of $P_{\mathrm{w}}, P_{\mathrm{xy}}$, or $P_{\mathrm{z}}$ because the charge balance is dominated by He-like ions and the $K_{\alpha}$ peak in the $\mathrm{Si}(\mathrm{Li})$ spectrum

(used to normalize intensities and determine $I_{\text {meas }}^{\text {mica }} / I_{\text {meas }}^{\text {TAP }}$ ) is therefore almost exactly proportional to $n_{\mathrm{He}}$.

While the theoretical and measured polarizations of $x y$ agree quite well, it is clear that, even with generous uncertainties, there is large disagreement for line $z$. There are three possibilities: the theoretical polarization is wrong, the TAP data are wrong, or the mica data are wrong. Based upon theory and the few other experimental results for He-like systems, it is quite unlikely that the theoretical value is significantly in error, and uniformity measurements for the TAP crystal leave little room for the $18 \%$ drop in reflectivity required to reconcile data and theory. The most likely explanation is that line $z$ was diffracted from an anomalously high-reflectivity region of the mica crystal, which is certainly possible given the much poorer statistical quality of the mica uniformity data. This will need to be checked in follow-up experiments.

In the meantime, it will be assumed that all data collected with the TAP crystal are valid and that theoretical polarizations are indeed accurate. The final section of this chapter explains how theoretical polarizations are used to obtain true line intensities from experimental spectra.

\subsection{Intensity Correction Factors}

Because of polarization-dependent spectrometer efficiency and nonisotropic emission, X-ray line intensities recorded by the FCS are not the same as those emitted 
from a collection of ions excited by random-direction electron collisions. Measured intensities must therefore be adjusted to account for these and any other instrumental effects. Ignoring geometrical efficiencies that are the same for all lines when observed simultaneously (such as the number of trapped ions and the solid angle acceptance of the spectrometer), the measured intensity of a line is given by

$$
I^{\text {meas }}=T A\left(I_{\|} R_{\|}+I_{\perp} R_{\perp}\right)
$$

where $T$ is the net transmision through the spectrometer and detector windows and $A$ is the fraction of $\mathrm{X}$-rays transmitted through the windows that are absorbed by the PSPC detector gas. For $\mathrm{P} 10$ gas $(90 \% \mathrm{Ar})$ at 1 atm and with an $8-\mathrm{mm}$ absorption depth, all the He-like Ne lines are completely absorbed so $A=1$. From equation $6.30, I_{\|}$and $I_{\perp}$ can be written in terms of $P$ so that equation 6.36 can be written as

$$
I^{\text {meas }}=\frac{1}{2} T I\left(90^{\circ}\right) R_{\mathrm{fl}}\left[(1+P)+(1-P)\left(\frac{R_{1}}{R_{1}}\right)\right]
$$

where $I\left(90^{\circ}\right)$ is the line intensity emitted from EBIT at an angle of $90^{\circ}$, equal to the sum of $I_{\|}$and $I_{\perp}$. The effect of nonisotropic emission is given by equation 6.17 so that the emitted intensity averaged over all space is equal to

$$
I^{4 \pi}=\frac{(3 \pm P) 2}{3 T R_{\|}} \frac{1}{(1+P)+(1-P)\left(\frac{R_{1}}{R_{1}}\right)} I^{\text {meas }}
$$

where the "+" is for M1 lines and the "-" is for E1 lines. To make comparisons easier, the intensity of any line $l, I_{1}^{4 \pi}$, can be normalized to the value of $I^{4 \pi}$ for line $w$ with zero polarization (meaning that $I_{W} 4 \pi=I_{W}$ meas when $w$ is unpolarized), so that

$$
I_{l}^{4 \pi}=I_{l}^{\text {meas }}\left(\frac{3 \pm P_{l}}{3}\right)\left(\frac{T_{w}}{T_{l}}\right)\left(\frac{R_{l}^{w}}{R_{l l}^{l}}\right) \frac{1+\left(\frac{R_{l}^{w}}{R_{l}^{w}}\right)}{\left(1+P_{l}\right)+\left(1-P_{l}\right)\left(\frac{R_{1}^{l}}{R_{l}^{l}}\right)} .
$$


For $q r$, which is a blend of two lines with different polarizations $\left(P_{\mathrm{r}}=0\right)$, we simply note that $I_{\mathrm{qr}}=I_{\mathrm{q}}+I_{\mathrm{r}}$ (for both measured and $4 \pi$ intensities) and use equation 6.39 to obtain

$$
\begin{aligned}
& I_{q r}^{4 \pi}=I_{q r}^{\text {meas }}\left(\frac{I_{q r}^{4 \pi}}{I_{q}^{4 \pi}}\right)\left(\frac{3-P_{l}}{1}\right)\left(\frac{T_{w}}{T_{q r}}\right)\left(\frac{R_{l}^{w}}{R_{l}^{q r}}\right) \times \\
& \frac{1+\left(\frac{R_{1}^{w}}{R_{1}^{*}}\right)}{3\left[\left(1+P_{q}\right)+\left(1-P_{q}\right)\left(\frac{R_{I}^{q r}}{R_{1}^{q}}\right)\right]+\left(\frac{I_{r}^{4 \pi}}{I_{q}^{4 \pi}}\right)\left(3-P_{q}\right)\left[1+\left(\frac{R_{r}^{q r}}{R_{l}^{q r}}\right)\right]}
\end{aligned}
$$

where $I_{r}^{4 \pi} / I_{q}^{4 \pi}=0.430$ at all energies (as explained in Chapter 8). The transmission of $w$ through the FCS and detector windows (a total of $4 \mu \mathrm{m}$ of polypropylene, $1 \mu \mathrm{m}$ of polyimide, and $300 \AA$ of aluminum) is $1.028,1.055$, and 1.0685 times that for lines $x y, q r$, and $z$, respectively. According to calculations by Gullikson [40], for TAP $R_{\|}$is constant for all $K_{\alpha}$ lines to one part in 3000 , while $R_{\perp} / R_{\|}$is equal to 0.2444 for $w$, 0.2362 for $x y, 0.2286$ for $q r$, and 0.2249 for $z$. As discussed in section 6.3.2, however, $R_{\|}$varies with position along the TAP crystal, so that $\mathrm{R}_{\|}{ }^{\mathrm{w}} / \mathrm{R}_{\|} \mathrm{xy}=1 / 1.027, \mathrm{R}_{\|} \mathrm{w} / \mathrm{R}_{\|} \mathrm{q}^{\mathrm{r}}=$ $1 / 1.053$, and $R_{\|}{ }^{w} / R_{\|}{ }^{2}=1 / 1.065$. Inserting these values into equations 6.39 and 6.40 yields

$$
\begin{aligned}
& I_{w}^{4 \pi}=I_{w}^{\text {meas }} \frac{\left(1-\frac{1}{3} P_{w}\right)}{\left(1+0.6072 P_{w}\right)}, \\
& I_{x y}^{4 \pi}=I_{x y}^{\text {meas }} \frac{1.008\left(1-\frac{1}{3} P_{x y}\right)}{\left(1+0.6179 P_{x y}\right)}, \\
& I_{q r}^{4 \pi}=I_{q r}^{\text {meas }} \frac{1.015\left(1-\frac{1}{3} P_{q}\right)}{\left(1+0.3388 P_{q}\right)}, \\
& I_{z}^{4 \pi}=I_{z}^{\text {meas }} \frac{1.019\left(1+\frac{1}{3} P_{z}\right)}{\left(1+0.6328 P_{z}\right)} .
\end{aligned}
$$

Note that the intensities of $w, x y$, and $q r$ (for which the measured polarizations agree well with theory) are fairly sensitive to polarization uncertainties while the intensity of line $z$ (which has discrepant experimental and theoretical polarizations) is relatively 
insensitive. The difference arises because $z$ is an M1 transition, thus putting a plus sign rather than a minus sign in the numerator. These equations will be used extensively in Chapter 8 to analyze spectral data. 


\section{Chapter 7}

\section{Radiative Lifetime of the ${ }^{3} S_{1}$ State}

Measurements of radiative lifetimes of the metastable $1 \mathrm{~s} 2 \mathrm{~s}{ }^{3} S_{1}$ level in heliumlike ions span 16 orders of magnitude from neutral He to $\mathrm{Xe}^{52+}[5,24,25,31,35$ $37,42,51,58,64,86]$. These measurements provide important tests of atomic structure calculations and serve as benchmarks for use in density diagnostics of laboratory and astrophysical plasmas. A large gap between $\mathrm{Li}^{+}(\tau=58.6 \mathrm{sec})$ and $\mathrm{S}^{14+}(\tau=706 \mathrm{nsec})$ remains that cannot be plored with laser fluorescence techniques applicable to neutral or singly charged ions, or with the beam-foil method used on accelerators to study lifetimes less than several hundred nanoseconds. The structure of ions in this gap is strongly affected by electron correlations, and lifetime measurements can test the accuracy of theoretical wavefunctions in a regime where uncertainties introduced by relativity are small.

Here we describe a new technique to measure radiative rates of levels in highly charged ions which are too slow to be measured with beam-foil techniques. It relies on high-resolution $\mathrm{x}$-ray spectroscopy of stationary, electrostatically trapped ions interacting with a monoenergetic electron beam. Our method of excitation is the inverse of the beam-foil method, and the radiative lifetime is determined directly from the temporal decay of the photon signal instead of from its spatial variation along a beamline. We apply the technique to $\mathrm{Ne}^{8+}$ and determine the radiative lifetime of the ${ }^{3} S_{1}$ level to be $90.4 \pm 1.4 \mu \mathrm{sec}$. The relative precision of our measurement surpasses that of any other published ${ }^{3} S_{1}$ lifetime and for the first time challenges theory at the percent level. 
This experiment consisted of three separate measurements performed on the Livermore electron beam ion trap (EBIT). The beam energy was switched $(1-2 \mathrm{kHz})$ between 960 and $750 \mathrm{eV}$, above and below the $905-\mathrm{eV}$ excitation threshold of the ${ }^{3} S_{1}$ level. The higher beam energy excites only the $n=2$ level, so corrections for radiative cascades from higher levels (usually the largest source of error in beam-foil experiments) are unnecessary. No lines are excited at the lower energy.

A vacuum flat crystal spectrometer (FCS) recorded the resulting $2 \rightarrow 1$ spectrum, shown in Fig. 7.1, which includes the resonance line $w\left(1 s 2 p^{1} P_{1} \rightarrow 1 s^{2}{ }^{1} S_{0}\right)$, the intercombination blend of $x$ and $y\left(1 s 2 p^{3} P_{2,1} \rightarrow 1 s^{2}{ }^{1} S_{0}\right)$, the forbidden line $z\left(1 s 2 s^{3} S_{1}\right.$ $\left.\rightarrow 1 s^{2}{ }^{1} S_{0}\right)$, and the lithiumlike collisional satellite blend of $q$ and $r\left(1 s 2 s 2 p^{2} P_{3 / 2,1 / 2} \rightarrow\right.$ $\left.1 s^{2} 2 s^{2} S_{1 / 2}\right)$. The $x y$ blend is dominated by $y$, which is about 26 times as intense as $x$. We used a TAP crystal $(2 d=25.76 \AA$ ), and a one-dimensional position-sensitive proportional counter with a $4-\mu \mathrm{m}$ polypropylene window to detect the dispersed $\mathrm{x}$ rays. For two of the three measurements made, we also used a $\mathrm{Si}(\mathrm{Li})$ detector which provided a much higher countrate, although the much lower spectral resolution meant that $w, x y, q r$, and $z$ could not be individually resolved.

A data acquisition system recorded the spectrum as a function of time with a resolution of $1.6 \mu \mathrm{sec}$. The temporal behavior of the individual lines is shown in Figure 7.2. The origin of the time axis is defined as the point at which the electron beam energy is switched from 960 to $750 \mathrm{eV}$. The slew rate is $10 \mathrm{eV} / \mu \mathrm{sec}$, and it thus takes about $20 \mu \mathrm{sec}$ for excitation of $w$ to cease. Line $z$ and the $\mathrm{Si}(\mathrm{Li}) 2 \rightarrow 1$ peak, however, both show prominent exponential tails. A weaker tail also appears on the $x y$ blend. The $x y$ tail exists because of collisional quenching of the ${ }^{3} S_{1}$ lifetime at the effective electron density of the measurement, i.e., because of transfer of an electron from the $1 s 2 s^{3} S_{1}$ to $1 s 2 p^{3} P_{2,1,0}$ levels. The ${ }^{3} P_{2}$ and ${ }^{3} P_{0}$ levels immediately decay 


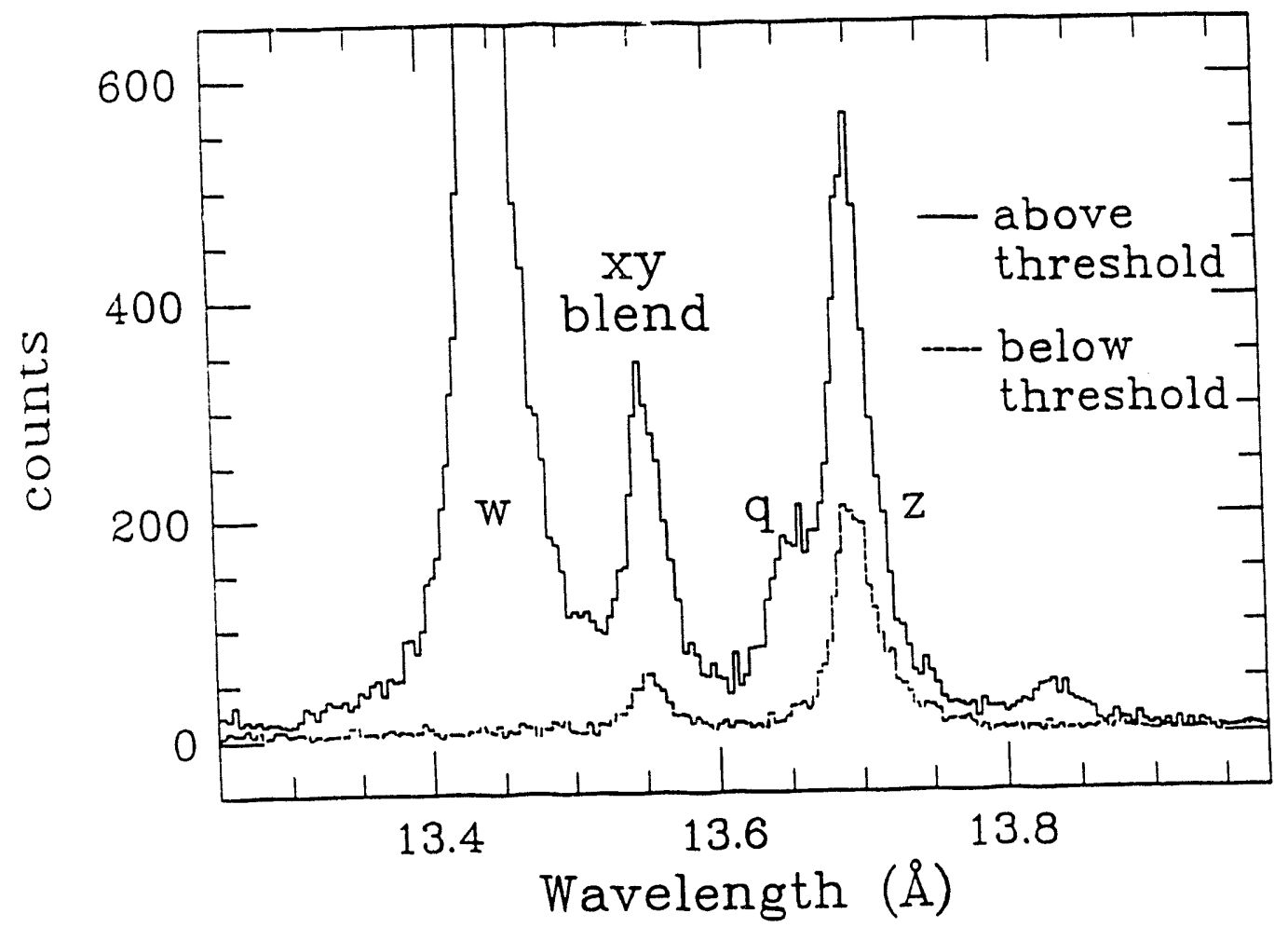

Figure 7.1. Spectra of He-like Ne as seen by the FCS. Note how only $x y$ and $z$ remain when the beam energy is lowered below the excitation threshold of $z$. Both spectra are integrations of the data shown in Figure 7.2: "above threshold" from time $=-150$ to 0 $\mu \mathrm{sec}$, and "below threshold" from 20 to $420 \mu \mathrm{sec}$. 
back to ${ }^{3} S_{1}$ (with branching ratios of $97.9 \%$ and $100 \%$, respectively), but the ${ }^{3} P_{1}$ level decays to ground via $y$ photon emission (branching ratio 96.5\%).

We can eliminate collisional quenching by reducing the beam current and thus the effective electron density. Alternatively, the effects of this collisional transfer may be easily accounted for by solving

$$
\frac{d n_{\text {mela }}}{d t}=-n_{\text {meta }}\left(\frac{1}{\tau_{z}}+\frac{1}{\tau_{\text {coll }-x y}}+\frac{1}{\tau_{\text {coll }-8}}\right)
$$

and

$$
\frac{d n_{x y}}{d t}=-\frac{n_{x y}}{\tau_{x y}}+\frac{n_{\text {mela }}}{\tau_{\text {coll }-x y}}
$$

where $n_{\text {meta }}$ is the number of metastable ${ }^{3} S_{1}$ ions, $n_{x y}$ is the number of ${ }^{3} P$ ions that decay to ground via $x$ and $y, \tau_{z}$ is the radiative lifetime of $z, \tau_{x y}$ is an average of the radiative lifetimes of $x$ and $y, \tau_{\text {coll-xy }}$ is the timescale for collisional redistribution from ${ }^{3} S_{1}$ to ${ }^{3} P$ that results in the production of an $x$ or $y$ photon, and $\tau_{\text {coll-g }}$ is the timescale for collisions from ${ }^{3} S_{1}$ to any other levels that then decay to ground. Solving these equations and using the fact that the radiative lifetimes for $x(0.44 \mu \mathrm{sec})$ [28] and $y(0.19 \mathrm{nsec})$ [30] are negligible compared to that for $z$ (the very small intensity of $x$ makes its lifetime effectively negligible), we obtain

$$
\frac{\tau_{z}}{\tau_{\text {coll }-x y}}=\frac{I_{x y}}{I_{z}}
$$

and

$$
\frac{1}{\tau_{\text {decay }}}=\frac{1}{\tau_{\text {decay }}}+\frac{1}{\tau_{\text {coll }-x y}}+\frac{1}{\tau_{\text {coll }-g}},
$$

where $I_{z}$ and $I_{x y}$ are line intensities during decay and $\tau_{\text {decay }}$ is the decay constant of the tails on both $x y$ and $z$. Combining Eqs. (7.3) and (7.4) we get 


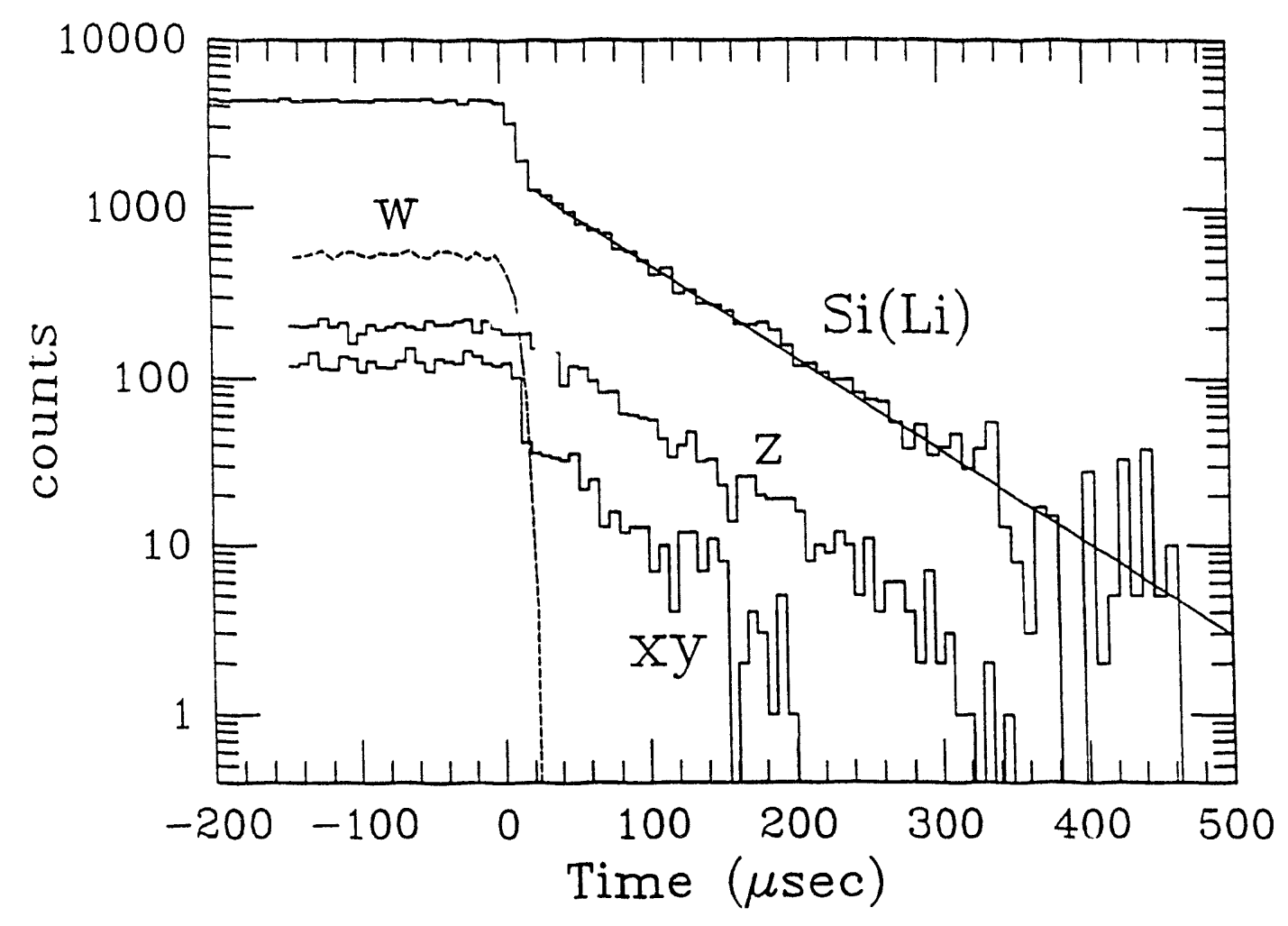

Figure 7.2. Line intensities as a function of time. The FCS data ( $w, x y$, and $z)$ are from measurement \#1, while the $\mathrm{Si}(\mathrm{Li})$ data is from measurement $\# 2$. Time $=0$ marks where the electron beam energy starts to drop from 960 to $750 \mathrm{eV}$. Excitation of the resonance line $w$ ceases after about $20 \mu \mathrm{sec}$. The exponential decay of the other lines beyond this point is apparent. Plotted data were background-subtracted. 


$$
\tau_{z}=\tau_{\text {decay }}\left[1+(1+k) \frac{I_{x y}}{I_{z}}\right]
$$

where the constant $k=\tau_{\text {coll-xy }} / \tau_{\text {coll-g }}$ " 1 is independent of density. Thus, the radiative lifetime of $z$ is equal to the decay constant of the exponential tails on $x y$ and $z$ multiplied by a correction factor arising from the collisional redistribution of ${ }^{3} S_{1}$ to other levels (mostly ${ }^{3} P$ ) that then decay to ground via photon emission.

We conducted measurements at the three effective electron densities listed in Table 7.1. Each density was determined from the ratio $I_{x y} I_{z}$ using a detailed model of the level populations in heliumlike neon constructed with the Hebrew UniversityLawrence Livermore Atomic Code package (HULLAC) [4]. The populations were calculated from a balance of all collisional and radiative processes connecting the $n=2,3$ excited levels as well as the ground state.

Starting at the point where excitation of $w$ had ceased, the decay curves of $x y$ and $z$ (using the sum of $x y$ and $z$ seen in the FCS data and the $x y z$ blend seen in the $\mathrm{Si}(\mathrm{Li})$ data) were fit with an exponential term $A e^{-t / \tau_{d e c a y}}$ plus a constant background. A typical fit is shown in Figure 7.2. The intensity ratios $I_{x y} I_{z}$ for each measurement were obtained by summing all counts in the exponential tails, and then correcting for small differences in spectrometer efficiency between $x y$ and $z$. The results are listed in Table 7.1, including $1 \sigma$-error estimates.

Looking at Eq. (7.5), the uncertainty in our determination of $\tau_{z}$ includes contributions from statistical errors in $\tau_{\text {decay }}$ and $I_{x y} I_{z}$, systematic uncertainties in the measurement of these quantities, and uncertainties in the theoretical calculation of $k$. As we show in the following discussion, all these possible errors are quite small, the largest arising from statistical uncertainties.

Because the electron beam density varies with radius and because a fraction of each ion's orbit may lie outside the beam [77], we actually measure an average of 
$\tau_{\text {decay }}$ over different densities. This means that the decay curve we fit is a superposition of exponentials with different decay constants. Likewise, the intensity ratio $I_{x y} / \lambda_{z}$ we measure is an average of many slightly different intensity ratios. Modeling this effect assuming a Gaussian beam profile, we find that fitting only one $\tau_{\text {decay }}$ to the decay curve and using the effective $I_{x y} / I_{z}$ will lead to an underestimate of $\tau_{\mathrm{z}}$. We correct for this by increasing $\tau_{\text {decay }}$ by $0.16(0.09,0.03) \mu \mathrm{sec}$ for measurement $\# 1$ (\#2,\#3) and including an uncertainty of equal magnitude, as shown in Table 7.1.

The interpretation of $I_{x y} / I_{z}$ and $\tau_{\text {decay }}$ is also affected by the polarization and anisotropy of the emitted line radiation since electron collisions in EBIT are nearly unidirectional [39]. In particular, the FCS is polarization-sensitive because the reflectivities of the TAP crystal for the two different polarization components differ by a factor of 4.5 [38]. Using the formalism of Alder and Steffen [2] and magnetic sublevel calculations from the code of Zhang, Sampson, and Clark [87], we compute the polarization of $z$ at time $=0$ to be $-10.5 \%(-11.7 \%,-13.1 \%)$ in measurement $\# 1(\# 2$, \#3) and conservatively assume a $30 \%$ relative uncertainty. The degree of polarization and anisotropy decreases slowly in time (fastest for the highest density measurement) because of collisional redistribution. In contrast, $x$ and $y$ are virtually unpolarized because the colliding electrons that excite these lines have an energy more than 70 times the $10-\mathrm{eV}$ threshold for collisional transfer from ${ }^{3} S_{1}$ to the ${ }^{3} P$ levels. Thus we determine that the apparent values of $I_{x y} I_{z}$ are too large by $2.5 \%, 3.1 \%$, and $3.8 \%$, respectively. Correspondingly, the apparent values of $\tau_{\text {decay }}$ are too high by $0.56 \mathrm{msec}$ for measurement \#1 and too low by $0.45(0.32) \mu \mathrm{sec}$ for measurement \#2 (\#3).

The apparent $\tau_{\text {decay }}$ may also be affected by the loss of ${ }^{3} S_{1}$ states due to recombination and ion escape from the trap region. We estimate the overall timescale for these losses by observing Lyman- $\alpha$ emission from hydrogenlike neon ions under similar conditions of electron density and neutral gas pressure. A population of $\mathrm{H}$-like 
TABLE 7.1. Results of lifetime measurements and associated uncertainties.

\begin{tabular}{|c|c|c|c|}
\hline Measurement & $\# 1$ & $\# 2$ & \#3 \\
\hline Spectrometer & FCS & $\mathrm{Si}(\mathrm{Li})$ and $\mathrm{FCS}$ & $\mathrm{Si}(\mathrm{Li})$ and $\mathrm{FCS}$ \\
\hline Electron density $\left(\mathrm{cm}^{-3}\right)$ & $8.7 \times 10^{11}$ & $5.7 \times 10^{11}$ & $2.9 \times 10^{11}$ \\
\hline \multicolumn{4}{|l|}{$I_{x y} / I_{z}$} \\
\hline Fit results & $0.218 \pm 0.010$ & $0.142 \pm 0.014$ & $0.073 \pm 0.011$ \\
\hline Corr. for spect. effic. & $+0.006 \pm 0.004$ & $+0.004 \pm 0.003$ & $+0.002 \pm 0.001$ \\
\hline Corr. for polarization & $-0.005 \pm 0.002$ & $-0.004 \pm 0.001$ & $-0.003 \pm 0.001$ \\
\hline Final value & $0.218 \pm 0.012$ & $0.141 \pm 0.014$ & $0.072 \pm 0.011$ \\
\hline \multicolumn{4}{|l|}{$\tau_{\text {decay }}(\mu \sec )$} \\
\hline Fit results & $74.33 \pm 2.28$ & $78.75 \pm 1.55$ & $83.58 \pm 1.98$ \\
\hline Timing calibration & $0.00 \pm 0.38$ & $0.00 \pm 0.50$ & $0.00 \pm 0.53$ \\
\hline Corr. for $n_{e}$ profile & $+0.16 \pm 0.16$ & $+0.09 \pm 0.09$ & $+0.03 \pm 0.03$ \\
\hline Corr. for polarization & $-0.56 \pm 0.17$ & $+0.45 \pm 0.14$ & $+0.32 \pm 0.10$ \\
\hline Corr. for ion loss & $+0.05 \pm 0.05$ & $+0.05 \pm 0.05$ & $+0.05 \pm 0.05$ \\
\hline Final value & $73.98 \pm 2.32$ & $79.34 \pm 1.64$ & $83.98 \pm 2.05$ \\
\hline \multicolumn{4}{|l|}{$\tau_{z}(\mu \sec )$} \\
\hline Using Eq. (5) & $90.27 \pm 2.96$ & $90.65 \pm 2.20$ & $90.11 \pm 2.39$ \\
\hline Uncertainty in $\mathrm{k}$ & $0.00 \pm 0.03$ & $0.00 \pm 0.02$ & $0.00 \pm 0.01$ \\
\hline Final value & $90.27 \pm 2.96$ & $90.65 \pm 2.20$ & $90.11 \pm 2.39$ \\
\hline
\end{tabular}


ions was first created by raising the beam energy above the ionization potential of $\mathrm{He}$ like ions $(1196 \mathrm{eV})$ but below that for ionization to tare nuclei $(1362 \mathrm{eV})$. The beam energy was then dropped to just above the excitation energy of Ly- $\alpha(1022 \mathrm{eV})$. Under these conditions, Ly- $\alpha$ intensity is proportional to the number of $\mathrm{H}$-like ions in the trap, and can be used to measure the loss rate of H-like ions. The Ly- $\alpha$ emission was then observed for $30 \mathrm{msec}$ with no appreciable decrease in intensity. We conclude that the timescale for loss of excited He-like ions is at least $60 \mathrm{msec}$, and thus the required correction to $\tau_{\text {decay }}$ must be no more than $0.10 \mu \mathrm{sec}$.

The final values for $\tau_{\text {decay }}$ and $I_{x y} / I_{z}$ are listed in Table 7.1. Their uncertainties are calculated from the quadrature sum of statistical errors and the correction term uncertainties discussed above, including a $0.51 \%(0.63 \%)$ uncertainty in the timing calibration for measurement \#1 (\#2 and \#3).

The constant $k$, which appears in Eq. (7.5), depends on the timescale for collisions from ${ }^{3} S_{1}$ to levels that then decay to ground other than through $x$ or $y$. We calculated this with the HULLAC modeling package which yields a value of 0.0082 , with an uncertainty of $20 \%$. Using this value, we obtained the values of $\tau_{z}$ shown in the Table.

Taking a weighted average we obtain a final result of $\tau_{z}=90.37 \pm 1.42 \mu \mathrm{sec}$. This is somewhat less than the value of $92.0 \mu \mathrm{sec}$ calculated by Drake [29]. Previously measured values of the ${ }^{3} S_{1}$ lifetime in higher- $Z$ ions $[5,24,25,31,35$ $37,42,58]$ are also systematically lower than Drake's, as shown in Figure 7.3, although their larger relative experimental uncertainties limit the significance of the disagreement with theory. More recently calculated values of $\tau_{\mathrm{z}}$, by Johnson and Lin [45], are in better agreement with experimental data. In particular, they report a $91.1-\mu \mathrm{sec}$ lifetime for neon, in good agreement with our result. 


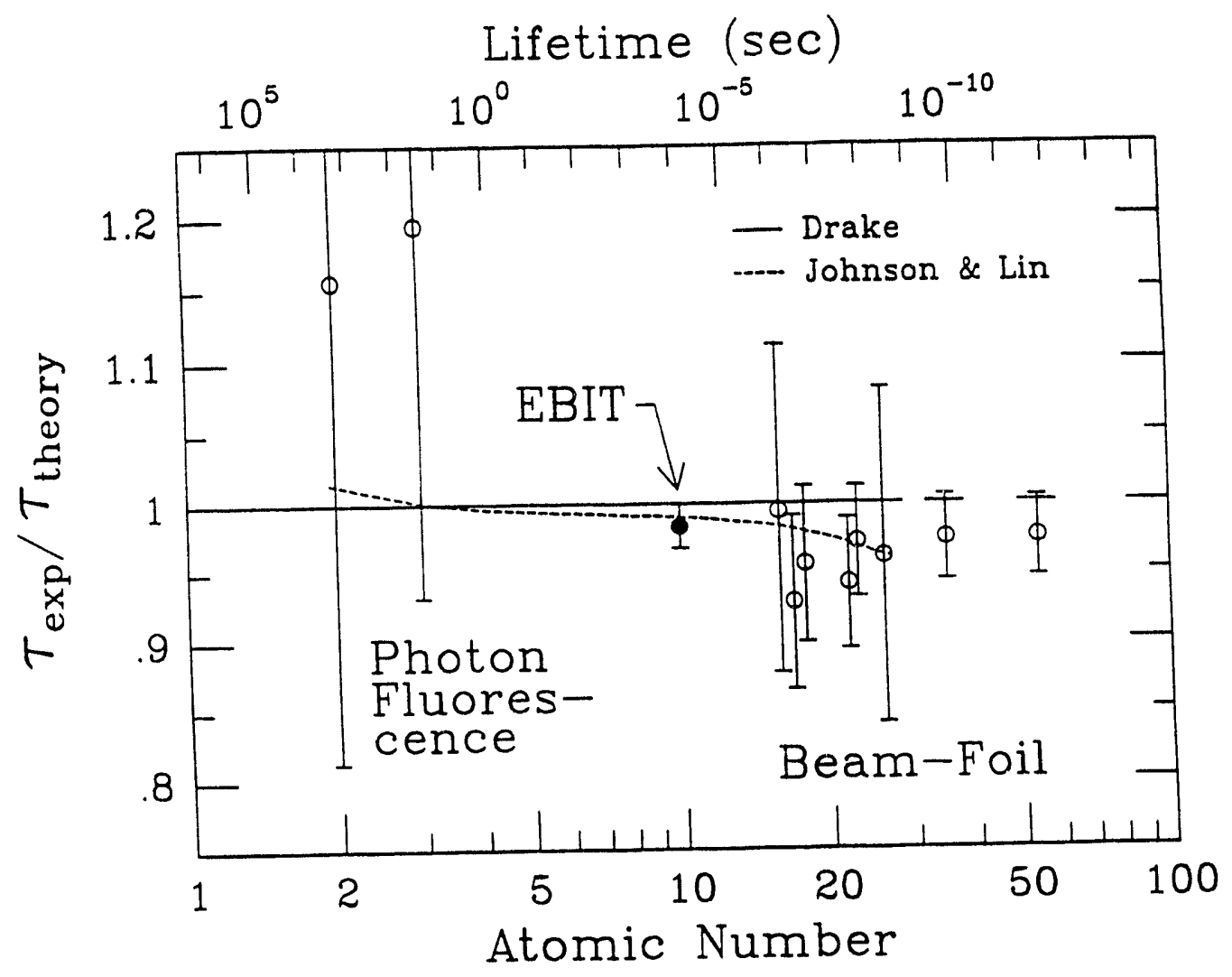

Figure 7.3. Experimental versus theoretical lifetimes for $z$, with labels indicating which method was used for each measurement. For consistency, theoretical lifetimes are taken from Drake $[31,29]$, except for xenon $(Z=54)$, for which we use the average of the six theoretical results quoted in the experimental measurement paper [58]. Experimental values are taken from Refs. $86(\mathrm{He}), 51(\mathrm{Li}), 5(\mathrm{~S}, \mathrm{Cl}), 42(\mathrm{Ar}), 35(\mathrm{Ti})$, $37(\mathrm{~V}, \mathrm{Fe}), 31(\mathrm{Br})$, and $58(\mathrm{Xe})$. Error bars are as quoted in each reference and reflect $68 \%$ confidence limits, except $\mathrm{He}, \mathrm{Li}$, and $\mathrm{Ar}$, for which they are higher. 


\section{Chapter 8}

\section{Analysis and Results}

This chapter presents the analysis of data which were obtained as described in Chapter 6. Relative cross sections are derived for electron impact excitation (including cascades) of all He-like $K_{\alpha}$ lines, as well as the cross section for production of line $z$ by innershell ionization of Li-like Ne. The cross section for collisional transfer from $2^{3} S_{1}$ to $2^{3} P$ was also measured. Section 8.1 deals with dielectronic recombination at energies below the EIE threshold and discusses how wavelengths and strengths of $K L L$ satellites were measured. Sections 8.2 and 8.3 cover analysis of the low- and high-energy data, and the final section applies these results to density and temperature diagnostics. In each section, experimental results are compared with theory.

\subsection{Dielectronic Recombination Satellite Lines}

Dielectronic recombination spectra, including the KLL band which lies lowest in energy, were obtained using the Event Mode operation and data acquisition system as described in Chapter 6. As mentioned in Chapter 1, DR satellites can be used as temperature diagnostics or as indicators of nonthermal energy distributions. In astrophysical applications, DR is most important for Fe because the relative strength of DR satellites increases with atomic number $Z$, thus filling the $\mathrm{Fe} K_{\alpha}$ spectrum with strong satellite lines. Even in low- $Z$ systems, however, DR satellites play an important role since they often blend with other non-satellite lines which have diagnostic uses. 
In particular, high- $n$ satellites always blend with their parent line and must be taken into account for proper interpretation of a given spectrum.

As explained in Chapter 2, dielectronic satellite lines are produced by the radiative stabilization of doubly excited, or autoionizing, levels populated by the radiationless, resonant, dielectronic capture of a free electron. For DR of He-like ions (to form Li-like ions) the process can be written as

$$
1 s^{2}+e^{-} \rightarrow 1 s 2 \ln l^{\prime} \rightarrow 1 s^{2} n l^{\prime}+h v^{\prime} .
$$

The spectator $n l^{\prime}$ electron partially shields the nuclear charge, so the photon energy $h v^{\prime}$ of the satellite line is slightly less than the energy $h v$ of the heliumlike transition

$$
1 s 2 l \rightarrow 1 s^{2}+h v
$$

DR satellite lines are therefore slightly shifted toward longer wavelengths, relative to their parent lines; the shift is largest for $n=2$ spectator electrons and approaches zero as $n \rightarrow \infty$. Likewise, the electron energy required to produce the $h v^{\prime}$ photon via DR is always less than the energy required for direct excitation, but approaches the EIE threshold energy as $n \rightarrow \infty$. This is because the dielectronic process makes use of potential energy associated with the recombining electron.

These two features, the offsets in electron energy and photon wavelength, can be seen in Figure 8.1, which is a plot of He-like Ne spectra obtained using the Event Mode system. The vertical axis is electron energy and the horizontal is wavelength. The electron beam energy was ramped between 600 and $1200 \mathrm{eV}$. For comparison, the direct excitation thresholds for $w, x y$, and $z$ are 922,916 , and $905 \mathrm{eV}$, respectively. Lines $q$ and $r$ are collisional satellites of $w$ resulting from inner-shell EIE of Li-like $\mathrm{Ne}$, with thresholds of $908 \mathrm{eV}$. Line $\beta$ is the corresponding Be-like satellite and a very faint B-like satellite (labeled $B$ ) can also be seen. 


\section{LII}

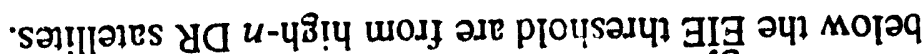

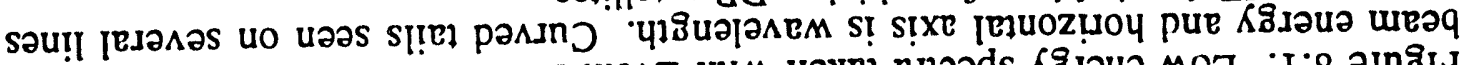

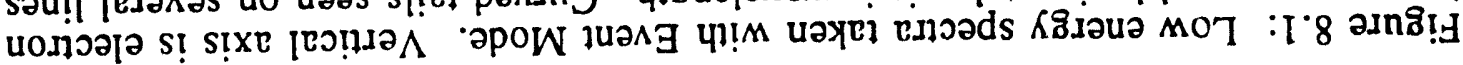

\section{$(\forall)$ чұбиәәәлем}

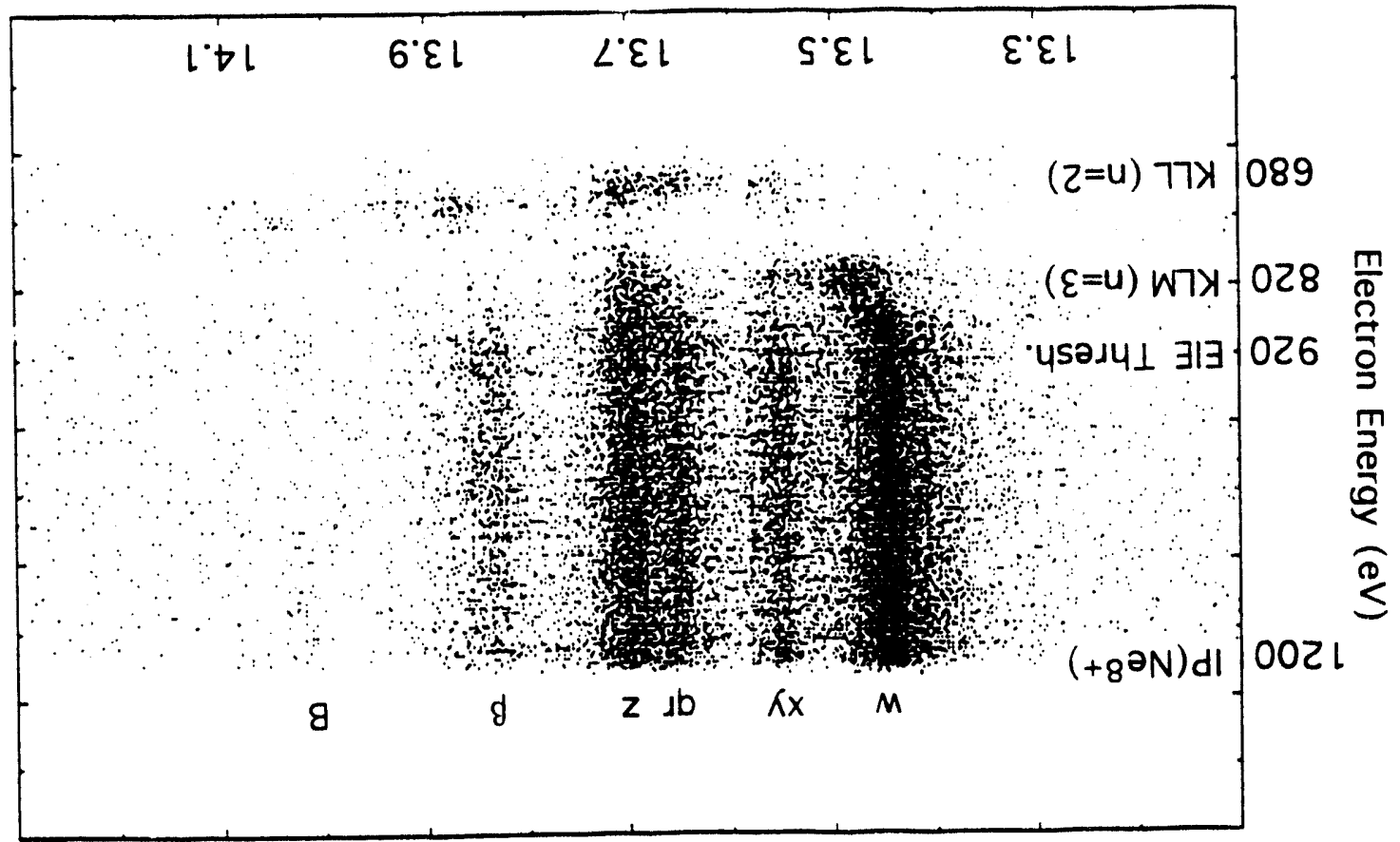


Note the prominent curved tails on $w$ and $q r$ that extend below threshold. These are DR satellite lines. Weaker tails can also be seen on $x y$ and $\beta$. Although DR is a resonant process, the electron beam energy spread smears out the individual high- $n$ resonances. The $K L M$ band ( $n=3$ spectator electron) at the bottom of the curved tails (about $820 \mathrm{eV}$ ) is partially resolved while the $K L L$ band $(n=2)$ around $680 \mathrm{eV}$ is quite distinct. The relatively strong $K L L$ satellites below about $13.8 \AA$ are Li-like satellites (which is why they mostly have wavelengths near those of the Li-like satellites $q$ and $r$ ) while satellites with $\lambda>13.8$ are Be-like. Some weak B-like $K L L$ satellites can be seen beyond about $14 \AA$.

\subsubsection{Fluorescence Yields}

The autoionizing states which produce satellite lines can either autoionize or undergo radiatively stabilizing transitions. The intensity of a satellite line therefore depends upon the relative rates of these competing processes. The fluorescence yield $W$ of an autoionizing state is defined as the fraction of the time that the state decays radiatively and is thus just a branching ratio. In the general case, several radiative transitions may be possible, so the fluorescence yield for a given satellite (from an autoionizing state to a final state) is given by

$$
W_{s}=W_{a f}=\frac{A_{a f}^{\text {Rad }}}{\sum_{j} A_{a j}^{\text {Rad }}+\sum_{k} A_{a k}^{\text {Auto }}}
$$

where $A_{a f}^{\text {Rad }}$ is the rate (units of $\sec ^{-1}$ ) for radiative decay from the autoionizing state la) to lower state $\mid f$, the first summed term in the denominator represents the total rate of radiative decay from state $|a\rangle$, and the second sum extends over all levels which are 
populated by autoionization of level $|a\rangle$ (for instance, a $1 s 2 l 2 l$ state can only autoionize to $1 s^{2}$, but a $1 s 3 l 4 l$ state can autoionize to $1 s 3 l, 1 s 2 l$, or $\left.1 s^{2}\right)$.

Fluorescence yields are necessary to compute the effective innershell EIE cross sections for the Li-like satellites $q$ and $r$. The effective cross section for the $q r$ blend is

$$
\sigma_{q r}=\sigma_{q}+\sigma_{r}=W_{q} \sigma_{q-\text { state }}+W_{r} \sigma_{r-\text { slate }}
$$

where $\sigma_{\mathrm{q} \text {-state }}$ and $\sigma_{\mathrm{r} \text {-state }}$ are the cross sections for excitation of the states that decay to produce $q$ and $r$. Lines $q$ and $r$ arise from essentially identical upper levels; the only difference is that $J_{i}=3 / 2$ for $q$ and $J_{i}=1 / 2$ for $r$. Since they are excited from the same $\mathrm{Li}$-like ground state, on statistical grounds one would expect the ratio $\sigma_{\mathrm{r} \text {-state }} \sigma_{\mathrm{q} \text {-state }}$ to be $1 / 2$. Reed [72] has calculated the excitation cross sections for all 16 autoionizing Li-like Ne levels of the type $1 s 2 l 2 l^{\prime}$ and finds that $\sigma_{\mathrm{r} \text {-state }} / \sigma_{\mathrm{q} \text {-state }}$ is indeed very near $1 / 2(0.493)$ for all energies. Several of the other 14 levels have cross sections nearly as large as for the states producing $r$ and $q$, but their $W$ factors are much smaller. Examples are lines $s$ and $t$, whose upper states have excitation cross sections almost half those of $q$ and $r$ (respectively), but whose fluorescence factors are a hundred times smaller. Lines $q$ and $r$ are therefore the only significant collisionally excited Li-like satellites in neon.

Fluorescence yields were taken from published tables. Vainshtein and Safronova [79] employed a perturbation technique on a basis of Coulomb functions using an expansion in powers of $1 / Z$ to calculate the radiative and Auger decay rates of all $16 \mathrm{Li}$-like $\mathrm{Ne} 1 \mathrm{~s} 2 \mathrm{~s} 2 l^{\prime}$ states. Chen [21] carried out calculations for $1 s 2 \ln ^{\prime} l$ ' states with $n^{\prime}=2$ and 3 (corresponding to $K L L$ and $K L M$ satellites) with configuration interaction using the multiconfigurational Dirac-Fock method (MCDF). Nilsen's calculations [65] for $1 s 2 l^{\prime} l^{\prime} \rightarrow 1 s 2 n^{\prime \prime} l^{\prime \prime}\left(n^{\prime}, n^{\prime \prime}=2,3,4\right)$ were based on relativistic MCDF 
bound states and distorted-wave Dirac continuum states. All three sources agree that $W_{\mathrm{r}} / W_{\mathrm{q}}=0.875$ to within one half percent. This means

$$
\sigma_{q r}=1.431 W_{q} \sigma_{q-\text { stase }}
$$

There was significant disagreement on the value of $W_{\mathrm{q}}$, however, with predictions varying by up to a factor of 1.8 from 0.351 (Chen), to 0.519 (Nilsen), to 0.637 (Vainshtein and Safronova). Results from the high energy data, described in section 8.3, will be used to determine the accuracy of the above predictions.

\subsubsection{Theoretical Resonance Strengths}

For a satellite line excited by dielectronic recombination, it is more useful to speak of a resonance strength rather than an excitation cross section. This is because cross sections for DR, which is a resonant process, are extremely energy-dependent, having Lorentzian shapes with natural widths of less than one $\mathrm{eV}$. If the cross section for excitation of a satellite line via $D R$ is written as $\sigma_{\mathrm{af}}\left(E-E_{\text {res }}\right)$, where $E_{\text {res }}$ is the resonant excitation energy, then the resonance strength $S_{\mathrm{af}}$ for the transition $|a\rangle \rightarrow \mid f$ is defined as

$$
S_{a f} \equiv \int_{-\infty}^{\infty} \sigma_{a f}\left(E-E_{\text {res }}\right) d E
$$

$S_{\mathrm{af}}$ can be thought of as the resonance strength for excitation of state la> from an initial state $|i\rangle$ times the fluorescence yield for decay to state $|f\rangle$, that is, $S_{\mathrm{af}}=S_{\mathrm{ia}} W_{\mathrm{af}}$. Because autoionization is just the inverse of excitation of an autoionizing state, by the principle of detailed balance, $S_{\mathrm{ia}}$ is proportional to $A_{a i}^{\text {Auto }}$, along with statistical weighting factors. The full equation for the resonance strength of a satellite line $s$ which results from the radiative decay of an autoionizing state $|a\rangle$ to a final state $|f\rangle$ is given by Beiersdorfer et al. [7] as 


$$
S_{s}=S_{a f}=\frac{g_{a}}{g_{i}} \frac{A_{a i}^{\text {Auto }} A_{a f}^{\text {Rad }}}{\sum_{j} A_{a j}^{\text {Rad }}+\sum_{k} A_{a k}^{\text {Auto }}} \frac{2 \pi^{2} a_{0}^{3} R_{y}^{2}}{E_{\text {res }}} \sqrt{\frac{m_{e}}{2 R_{y}}}
$$

where $g_{\mathrm{a}}$ is the statistical weight of the autoionizing level (equal to $2 J_{\mathrm{a}}+1$ ), $g_{\mathrm{i}}$ is the weight of the initial (ground state) level (equal to 1 for He-like ions and 2 for Li-like), $a_{0}$ is the Bohr radius, $R_{\mathrm{y}}$ is the Rydberg energy, and $m_{\mathrm{e}}$ is the electron mass. It is common to refer to a satellite intensity factor for each satellite transition, defined as

$$
Q_{d}=\frac{g_{a} A_{a i}^{\text {Auto }} A_{a f}^{\text {Rad }}}{\sum A_{a j}^{\text {Rad }}+\sum_{k} A_{a k}^{\text {Auto }}} \quad\left[\sec ^{-1}\right]
$$

so that equation 8.7 may be written as

$$
S_{s}=\left(2.475 \times 10^{-30}\right) \frac{Q_{d}}{g_{i} E_{\text {res }}} \quad\left[\mathrm{cm}^{2} \mathrm{eV}\right]
$$

where $E_{\text {res }}$ is in $\mathrm{eV}$.

Note that satellites are strongest when they have large radiative and autoionization rates. Because most autoionization rates are all about the same (within a factor of 100 or so), $S_{S}$ is largely determined by the radiative rate. Radiative rates are not much affected by spectator electrons, so lines emitted from states with short lifetimes (large $A_{r}$ ), such as the El lines $w$ and $q$, have strong satellites, while line $z$, emitted from the long-lived ${ }^{3} S_{1}$ state, has essentially no satellites (see Figure 8.1).

\subsubsection{Experimental Measurement of $K L L$ Resonance Strengths}

Resonance strengths can be measured on EBIT by normalizing the observed intensity of a DR satellite to the intensity of a line excited by EIE whose cross section is known as a function of energy. The observed intensity of a Li-like DR satellite is 


$$
I_{s}\left(E_{\text {ave }}\right)=v_{c} n_{e} n_{H e} S_{s} f\left(E_{\text {res }}-E_{\text {ave }}\right)
$$

where $E_{\text {ave }}$ is the average energy of the beam electrons and the function $f\left(E-E_{\text {ave }}\right)$ describes the energy distribution of the electrons. In Chapter $6, f$ was modeled as a Gaussian function with a FWHM of $50 \mathrm{eV}$. If the energy distribution function is normalized so that

$$
\int_{-\infty}^{\infty} f\left(E-E_{a v e}\right) d E=1
$$

then integrating both sides of equation 8.10 over all energies (experimentally, by sweeping the beam energy up and down across the resonance) yields

$$
I_{s}(\text { total })=v_{e} n_{e} n_{H e} S_{s} .
$$

Since the intensity of Li-like satellites is proportional to the He-like ion density, their strengths must be normalized to a He-like line excited by EIE. The obvious choice is line $w$, which has an intensity given by

$$
I_{w}\left(E_{\text {ave }}\right)=v_{e} n_{e} n_{H e} \sigma_{w}\left(E_{\text {ave }}\right) .
$$

Integrating over a range of energies, in this case from 920 to $1200 \mathrm{eV}$, then yields

$$
I_{w}(920-1200)=v_{c} n_{e} n_{H e} \int_{920}^{1200} \sigma_{w}\left(E_{a v e}\right) d E_{\text {ave }} .
$$

For this measurement, the beam current was held fixed so that $v_{e} n_{e}$ was constant. $I_{s}$ and $I_{\mathrm{W}}$ were measured at the same time so that $v_{e} n_{e} n_{H e}$ cancels out when equations 8.12 and 8.14 are divided, giving

$$
S_{s}=\frac{I_{s}(\text { total })}{I_{w}(920-1200)} \int_{920}^{1200} \sigma_{w}\left(E_{\text {ave }}\right) d E_{\text {ave }} .
$$




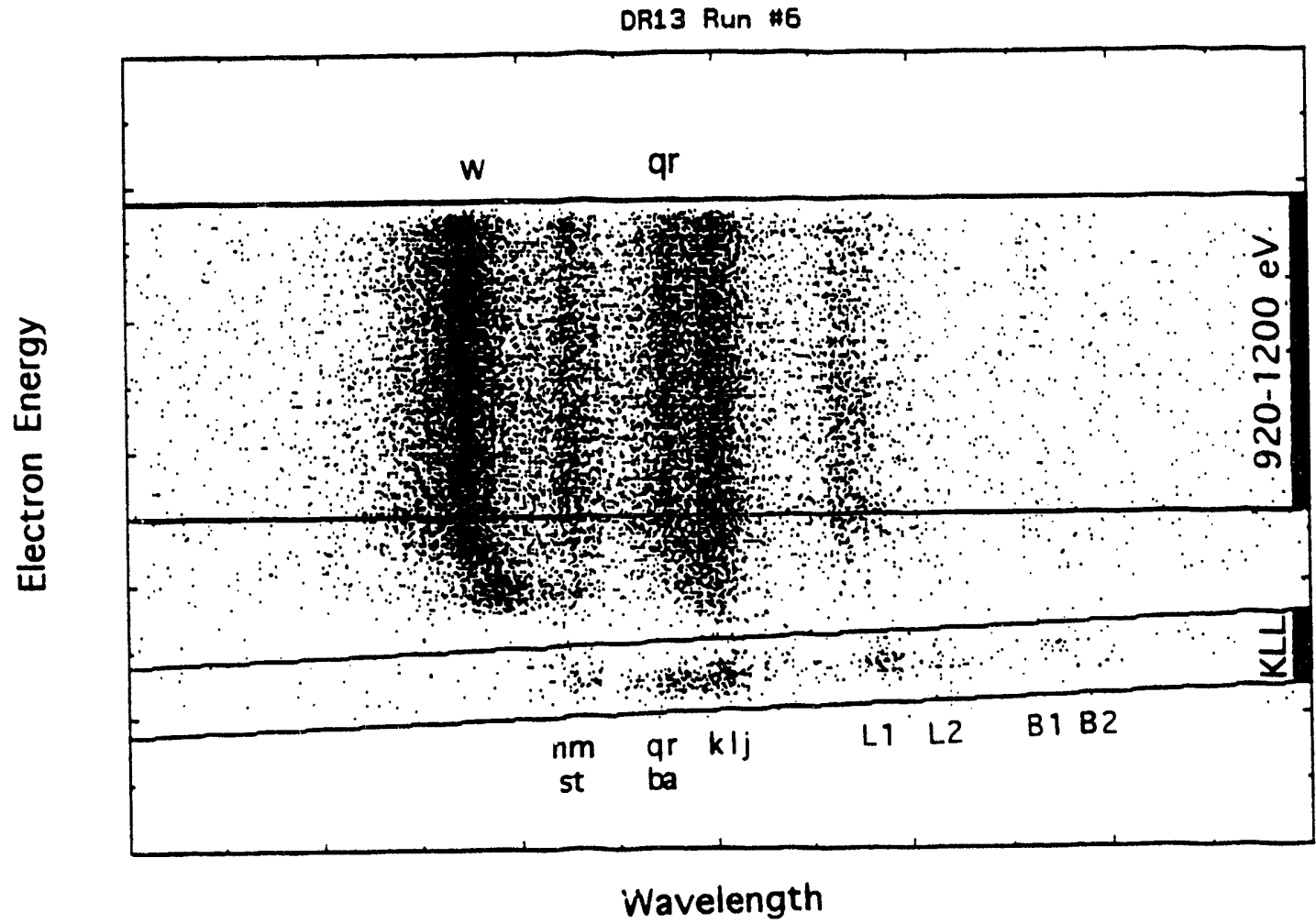

Figure 8.2: Energy windows used to determine DR resonance strengths. The KLL spectrum contained in the lower window is shown in Figure 8.3. Intensities of lines $w$ and $a r$ were summed from 920 to $1200 \mathrm{eV}$ for normalization of $\mathrm{Li}$-like and Be-like satellites. 
The resonance strength of Be-like satellites is given by the same formula, but by normalizing to the collisionally excited Li-like $q r$ blend.

$I_{\mathrm{s}}($ total) was found iur each DR satellite by collapsing the spectra contained in the window shown in Figure 8.2. The resulting spectrum is shown in Figure 8.3a. The integrated intensities of $w$ and $q r$ were found by summing all spectra above 920 $\mathrm{eV}$. The number of counts in each line or blend was determined by using the PKFIT program. Intensities were then corrected for instrumental and polarization effects using the appropriate version of equation 6.39. Li-like satellite polarizations were taken from Inal and Dubau [44] and adjusted to account for the electron beam's transverse velocity using equation 6.25 . Values of $E_{\text {res }}$ for each Li-like satellites were provided by Chen [22]. The average value of $P_{w}$ and the integral of $\sigma_{w}$ were computed using the smoothed cross sections described in Chapter 6.2. For the Be-like satellites, it was assumed that $P=+0.25$ (the polarization of $q r$ at threshold), reduced to 0.19 by transverse beam velocity effects at the average resonance energy of $700 \mathrm{eV}$, taken from Chen [23]. $P_{q}$ and $\int \sigma_{q r}$ were computed in the same manner as for $w$, using the fluorescence yield $W_{q}=0.637$ calculated by Vainshtein and Safronova [79]. Satellite wavelengths were also measured, using $w, x y$, and $z$ as reference lines with wavelengths of $13.4473,13.5530$, and $13.6990 \AA$, respectively, taken from Drake [27] and assuming that $I_{y} I_{x}=26$, as computed by Osterheld [66]. Results are shown in Table 8.1 for Li-like satellites, and Table 8.2 for Be-like satellites.

Theoretical resonance strengths for the Li-like lines were computed according to equation 8.9 using satellite intensity factors $\left(Q_{d} s\right)$ calculated by Nilsen [65], Chen [21], and Vainshtein and Safronova [79]. Strengths for the Be-like satelintes were derived from the results Chen [22], and wavelengths were taken from Chen and Crasemann [20]. It should be noted that although values of $Q_{d}$ are also listed by Chen and Crasemann, they are completely useless for determining the strengths of Be-like 

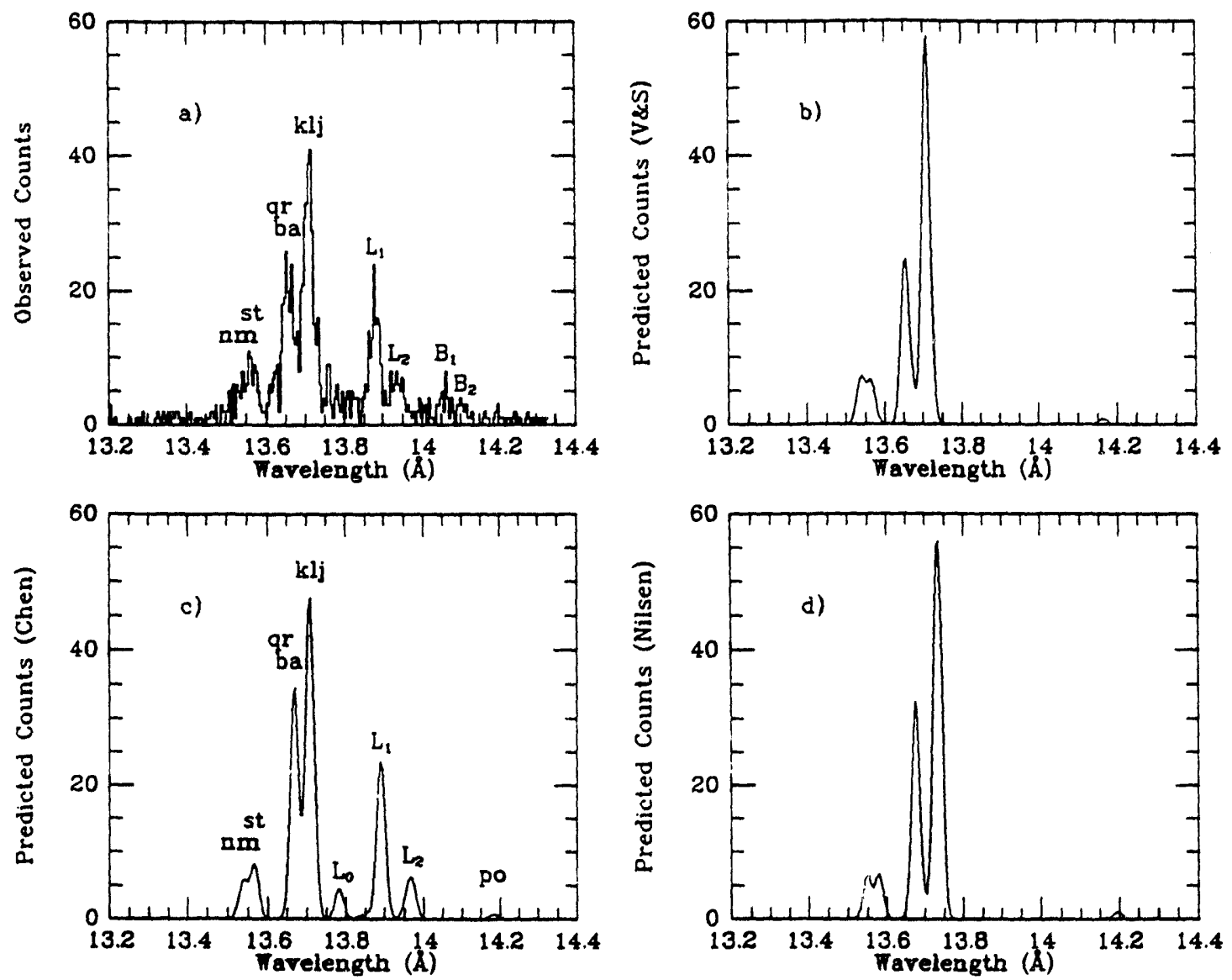

Figure 8.3: Experimental and theoretical $K L L$ spectra. Theoretical Be-like satellite spectra are not shown in $b$ or $d$ because they were not computed. 
satellite lines because they were calculated with the assumption that $A_{a i}^{\text {Auto }}=\sum_{k} A_{a k}^{\text {Auto }}$. While this is true for Li-like $1 s 2 l 2 l$ ' states, which virtually always autoionize to the single ground state of the He-like ion, Be-like $1 s 2 l 2 l$ ' $2 l^{\prime \prime}$ states autoionize to create a Li-like ion which has three possible ground states, namely $1 s^{2} 2 s^{2} s_{1 / 2}, 1 s^{2} 2 p 2 P_{1 / 2}$, and $1 s^{2} 2 p^{2} P_{3 / 2}$. The tabulated results of Chen and Crasemann [20] list only the total autoionization rate for each state, and not the individual rates for decay to the ground states of the Li-like ion. After being told of this problem, Chen immediately provided individual autoionization rates [22]. The resulting theoretical resonance strengths are presented in Tables 8.1 and 8.2 and were also used to predict what the observed spectra would look like after accounting for polarization and instrumental effects. The model spectra are shown in Figures $8.3 b, c$, and $d$.

Reasonably good agreement is found between theory and experiment for the Lilike satellites, although theoretical resonance strengths are on average $34 \%$ higher than measured values. Note that strengths predicted by the three theories typically vary by approximately $20 \%$. The wavelength results of Vainshtein and Safronova are clearly superior to the other theoretical predictions.

There is an estimated $10 \%$ uncertainty in the experimentally determined resonance strengths which arises from uncertainties in the assumed line polarizations. A source of error that is more difficult to estimate arises from changes in trap conditions, specifically the beam-ion overlap, as the electron beam sweeps up and down over a factor of two in energy. Since beam current was held fixed for this measurement, electron density increased as electrons moved more slowly at lower energies. This would tend to increase the beam-ion overlap as the electron space charge attracted ions more strongly. The result of this extra attraction, however, would be more trapped ions at low energies, thus increasing the apparent satellite intensities. Beiersdorfer et al. [7] estimated the increase at between 5 and $20 \%$ in their 
Table 8.1: Atomic data for principal $K L L$ dielectronic satellite transitions $1 \mathrm{~s} 212 l^{\prime} \rightarrow 1 \mathrm{~s}^{2} 2 l^{\prime}$ in Li-like $\mathrm{Ne}^{7+}$. Transitions are labeled in the notation of Gabriel [32] and are grouped to indicate blending. Experimental wavelengths were measured with respect to the He-like lines $w, x y$, and $z$ using wavelengths from Drake [27]. Numbers in parentheses denote the uncertainty in the last digit(s). Experimental resonance strengths were measured with respect to line $w$, using theoretical cross sections calculated by Reed [72]. Experimental values of $\lambda$ and $S$ are for blends.

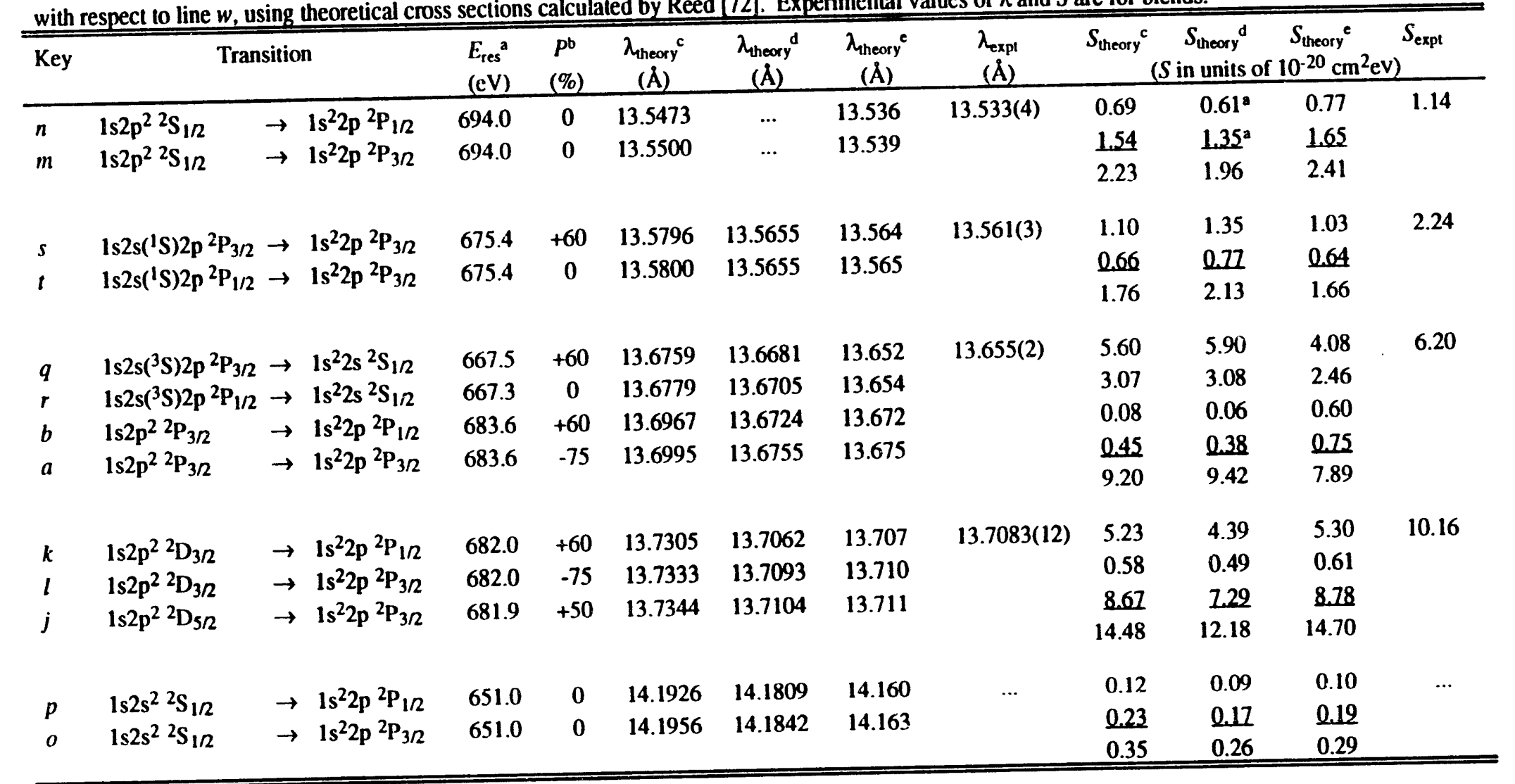

${ }^{\mathrm{a} C h e n, ~ p r i v a t e ~ c o m m u n i c a t i o n ~[22] . ~}$

bInal and Dubau [44]

cNilsen [65].

dChen [21].

e Vainshtein and Safronova [79]. 
Table 8.2: Atomic data for principal $K L L$ dielectronic satellite transitions $1 \mathrm{~s} 2 \mathrm{l}^{3} \rightarrow 1 \mathrm{~s}^{2} 2 \mathrm{l}^{2}$ in Be-like $\mathrm{Ne}^{6+}$. Theoretical values of $\lambda$ and $S$ are taken from Chen [21,22]. Transitions are grouped to roughly correspond with observed spectral features. Experimental resonance strengths were measured with respect to the collisionally excited Li-like satellite blend $q r$, using theoretical cross sections calculated by Reed [72] and fluorescence vields calculated by Vainshtein and Safronova [79].

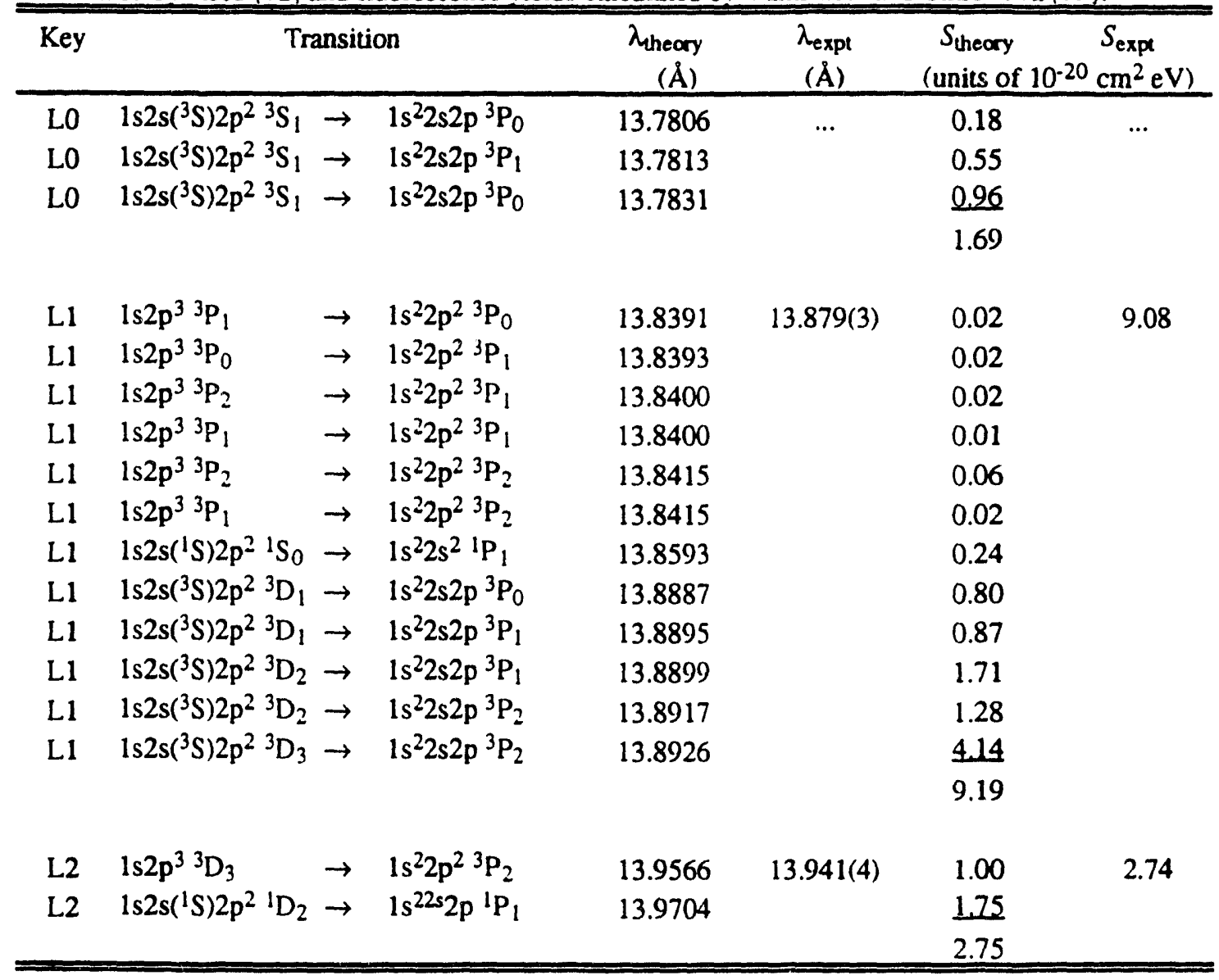


measurement of resonance strengths for $\mathrm{He}$-like $\mathrm{Fe}$, but at the very low energies required to study neon, beam instabilities might counteract some of this increase. Net experimental uncertainty of this measurement is estimated to be $20 \%$, which suggests that theoretical predictions are indeed too high.

Agreement between experiment and theory is excellent for the Be-like satellites, although the reader is reminded that the theoretical predictions of Vainshtein and Safronova [79] for $W_{q}$ and $W_{r}$ were used to compute $\sigma_{q r}$, which is used in Equation 8.15 to normalize the experimental resonance strengths. Agreement is not as good if the fluorescence factors computed by Chen [21] or Nilsen [65] are used instead. In particular, using Chen's value of 0.351 for $W_{q}$ (instead of 0.637 ) makes the experimental resonance strengths a factor of 1.8 larger than theory. Experimental error, even with the uncertainty in the exact polarization of the satellites (conservatively estimated at \pm 0.25 ) and some ambiguity in assigning individual radiative transitions to observed spectral features (because of errors in theoretically calculated wavelengths of Be-like transitions and some blending of lines), is no larger than $30 \%$.

\subsection{Low Energy Data}

The energy region within about $300 \mathrm{eV}$ of the roughly $900-\mathrm{eV}$ threshold for direct excitation of He-like neon $K_{\alpha}$ lines is of great importance, both because it generally contributes most of the neon emission in collisional plasmas (because of the Maxwellian distribution of electron energies and the fall-off in excitation cross sections at higher energies) and because of the complexity and structure in the $K_{\alpha}$

excitation cross sections near threshold. In addition to the $K L L$ DR resonances described above, there are higher order DR satellites ( $K L M, K L N, K L O+$ resonances) 
just below threshold, and resonant excitation peaks just above threshold. In addition, cascades add step-like features to the EIE cross sections up to the 1196-eV threshold for ionization of He-like ions.

As described in Chapter 5.2, the energy range between 750 and $1200 \mathrm{eV}$ was studied using the Event Mode system. In this second Event Mode measurement, the electron beam energy was ramped up and down, but at a much slower rate than for the first measurement (which was used to study the $K L L$ resonances) so that features in the cross section of the forbidden line $z$ (which is produced by the decay of a longlived atomic state) would not be smeared out. Data from this measurement are presented in the following section (8.2.1), and results for DR. EIE, and RE are compared with theory in section 8.2.2.

\subsubsection{Near-Threshold Line Intensities}

Above the EIE threshold of each line, but below the 1196-eV threshold for production of $\mathrm{H}$-like ions (and therefore below the threshold for significant innershell ionization of $\mathrm{Li}$-like $\mathrm{Ne}$ ), the intensities of lines $w, x y$, and $z$ are proportional to the number of He-like ions in the trap. (Above the $1107-\mathrm{eV}$ threshold for innershell ionization of Li-like neon, there is some contribution to the intensity of $z$, but as shown in section 8.3, the ISI cross section is still quite small below $1200 \mathrm{eV}$. In addition, there are fewer $\mathrm{Li}$-like than He-like ions in the trap, so the importance of ISI below $1200 \mathrm{eV}$ is negligible.) Likewise, the intensity of the $q r$ blend is proportional to the number of Li-like ions. In the low-density limit, the equations for line intensity are therefore

$$
\begin{aligned}
& I_{w}=v_{e} n_{e} n_{H e} \sigma_{w} \\
& I_{x y}=v_{c} n_{e} n_{H e} \sigma_{x y}
\end{aligned}
$$




$$
\begin{aligned}
& I_{z}=v_{e} n_{e} n_{H e} \sigma_{z} \\
& I_{q r}=v_{e} n_{e} n_{L i} \sigma_{q r}
\end{aligned}
$$

where $v_{e}$ is the electron velocity, $n_{e}$ is the electron density, $n_{H e}$ is the density of Helike ions, $n_{L i}$ is the density of Li-like ions, and the $\sigma s$ are the effective line excitation cross sections after cascades have been included (see Chapter 6).

In this experiment, as discussed before, the electron density is high enough that there is significant collisional transfer between the ${ }^{3} S_{1}$ level that produces line $z$ and the ${ }^{3} P$ levels that produce $x y$. The effect of this transfer on the intensities of $x y$ and $z$ was modeled in Chapter 6. If we define $\sigma(0)$ as the line excitation cross section as density approaches zero, $\sigma\left(n_{e}\right)$ as the effective cross section at density $n_{e}$, and $f_{n e t}$ as the net transfer of " $z$ intensity" to " $x y$ intensity", then the equations for $l_{x y}$ and $I_{z}$ may be written as

$$
\begin{gathered}
I_{x y}=v_{c} n_{e} n_{H e} \sigma_{x y}\left(n_{e}\right)=v_{c} n_{e} n_{H e}\left[\sigma_{x y}(0)+f_{n e t} \sigma_{z}(0)\right] \\
I_{z}=v_{e} n_{e} n_{H e} \sigma_{z}\left(n_{e}\right)=v_{e} n_{e} n_{H e} \sigma_{z}(0)\left[1-f_{\text {net }}\right] .
\end{gathered}
$$

This experiment measures the effective line excitation cross sections including the effects of collisional transfer, that is, the $\sigma\left(n_{e}\right)$ 's. The net transfer $f_{n e t}$ and the relative cross section for transfer as a function of energy will be determined from the high energy data in section 8.3, along with line excitation cross sections at zero density.

As described in Chapter 3, this experiment was designed to keep the electron density constant as a function of energy. Likewise, the beam energy is ramped up and down at a very rapid rate so that the ionization balance does not change, that is, the densities of He-like and Li-like ions are also constant. Since the electron velocity $v_{e}$ scales as $E^{1 / 2}$, the line intensity equations can now be written as

$$
I_{w}=C \sqrt{E} \sigma_{w}
$$




$$
\begin{aligned}
& I_{x y}=I_{w} \frac{\sigma_{x y}\left(n_{e}\right)}{\sigma_{w}} \\
& I_{z}=I_{w} \frac{\sigma_{\cdot}\left(n_{e}\right)}{\sigma_{w}} \\
& I_{q r}=I_{w} \frac{n_{L i}}{n_{H e}} \frac{\sigma_{q r}}{\sigma_{w}}
\end{aligned}
$$

where $C$ is a constant (equal to $\sqrt{\frac{2}{m_{e}}} n_{e} n_{H e}$ ). These equations form the basis of the analysis procedure used to derive relative line excitation cross sections as a function of energy.

As shown in Figure 8.4, a range of data between 730 and $1230 \mathrm{eV}$ was selected and then binned into 25 separate spectra, $20 \mathrm{eV}$ per bin. Data accumulated on the up and down sweeps were compared to confirm that corresponding energy bins covered the same range in each case. Each of the 25 spectra were then analyzed separately using the PKFIT program to determine the intensity of each line. DR satellites were treated as contributors to the intensity of their respective parent lines. Using the polarizations computed in Chapter 6 (see equation 6.26), the intensity of each line was corrected for instrumental and polarization effects. By dividing by $E^{1 / 2}$ and normalizing to the intensity of line $w$ at $1000 \mathrm{eV}$, the excitation cross section for each line is then equal to its normalized intensity times the cross section of $w$ at $1 \mathrm{keV}$. Results are shown in Figures 8.5 through 8.8 .

The 23 filled circle data points for each spectrum cover energies from 760 to $1200 \mathrm{eV}$. There are only 21 data points for $w$ tccause the amount of time spent in each of the two energy bins at the top of the energy sweep was somewhat less than the time-per-bin over the rest of the sweep. The observed intensities of $w$ at these two energies are therefore anomalously low (not shown), but the intensities of the other $K_{\alpha}$ lines relative to $w$ are still perfectly valid. There are additional data points at $1000 \mathrm{eV}$ and $1100 \mathrm{eV}$ (plotted with open squares) taken from steady state measurements at 


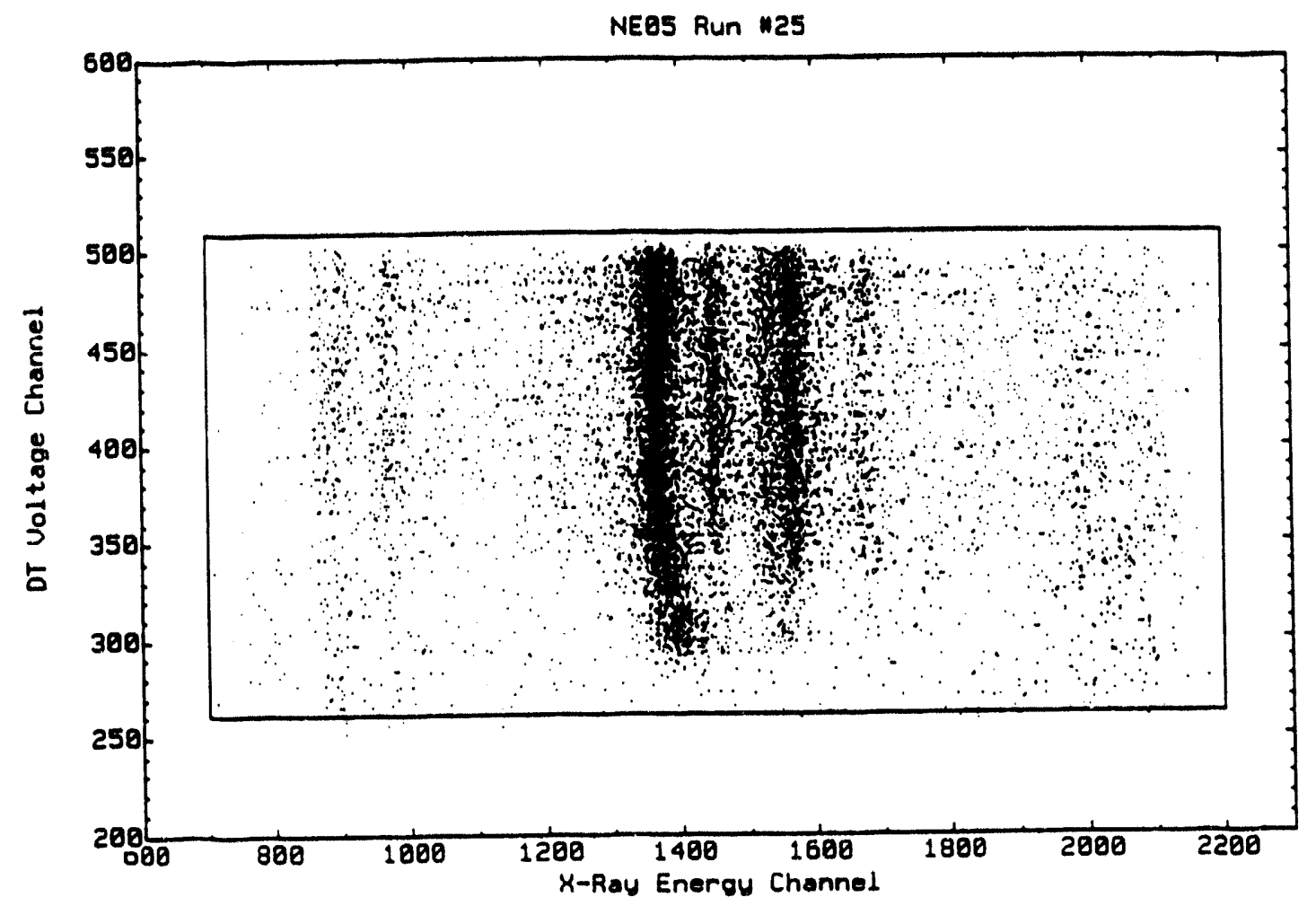

Figure 8.4: Event Mode spectra from 750 to $1200 \mathrm{eV}$. Boxed region was divided into 25 horizontal slices which were analyzed as separate spectra. 
those energies. The data at $1000 \mathrm{eV}$ are normalized so that $I_{w}=1$, as for the Event Mode data; the points at $1100 \mathrm{eV}$ are plotted relative to the theoretically predicted value for $w$ at $1100 \mathrm{eV}$. Steady-state results for $q r$ are not plotted because the ratio of He-like to Li-like ions was probably different from that during the Event Mode data run, and so comparisons cannot be made.

Error bars on the data points show statistical uncertainties and include contributions from background, as described in Chapter 6. Systematic errors are dominated by the uncertainties in localized crystal reflectivity and window transmissions, line polarization (usid for intensity corrections according to equation 6.39), and variations in trap conditions (such as electron and ion densities) as the beam energy sweeps up and down.

Variations in crystal uniformity, window transmission, and detector response are estimated to be at the $5 \%$ level or less. In the low-density limit, theoretical polarizations are probably accurate to a few percent, but the effects of collisional transfer on $P_{x y}$ and $P_{z}$ are not as well determined because of uncertainties in the collisional rate. As an example of the effects of polarization error, take $P_{w}=$ $0.60 \pm 0.04$ and $P_{x y}=-0.30 \pm 0.12$ after collisional transfer. Then apply equation 6.25 to correct for the effects of transverse electron velocity in the beam. assuming a $10 \%$ uncertainty in the correction factor. At $1 \mathrm{keV}$, the corrected polarizations then will be $P_{w}=0.47 \pm 0.07$ and $P_{x y}=-0.24 \pm 0.10$. These uncertainties carry through the intensity corrections for polarization (equations 6.41 and 6.42) to produce uncertainties in $I^{4 \pi}$ of roughly $7 \%$ for line $w$ and $10 \%$ for $x y$. Line $z$, being less affected by polarization uncertainties, has an uncertainty of 3 or $4 \%$ in its relative intensity. The $q r$ blend, which includes an intrinsically unpolarized line, has an intensity uncertainty of approximately $6 \%$. 

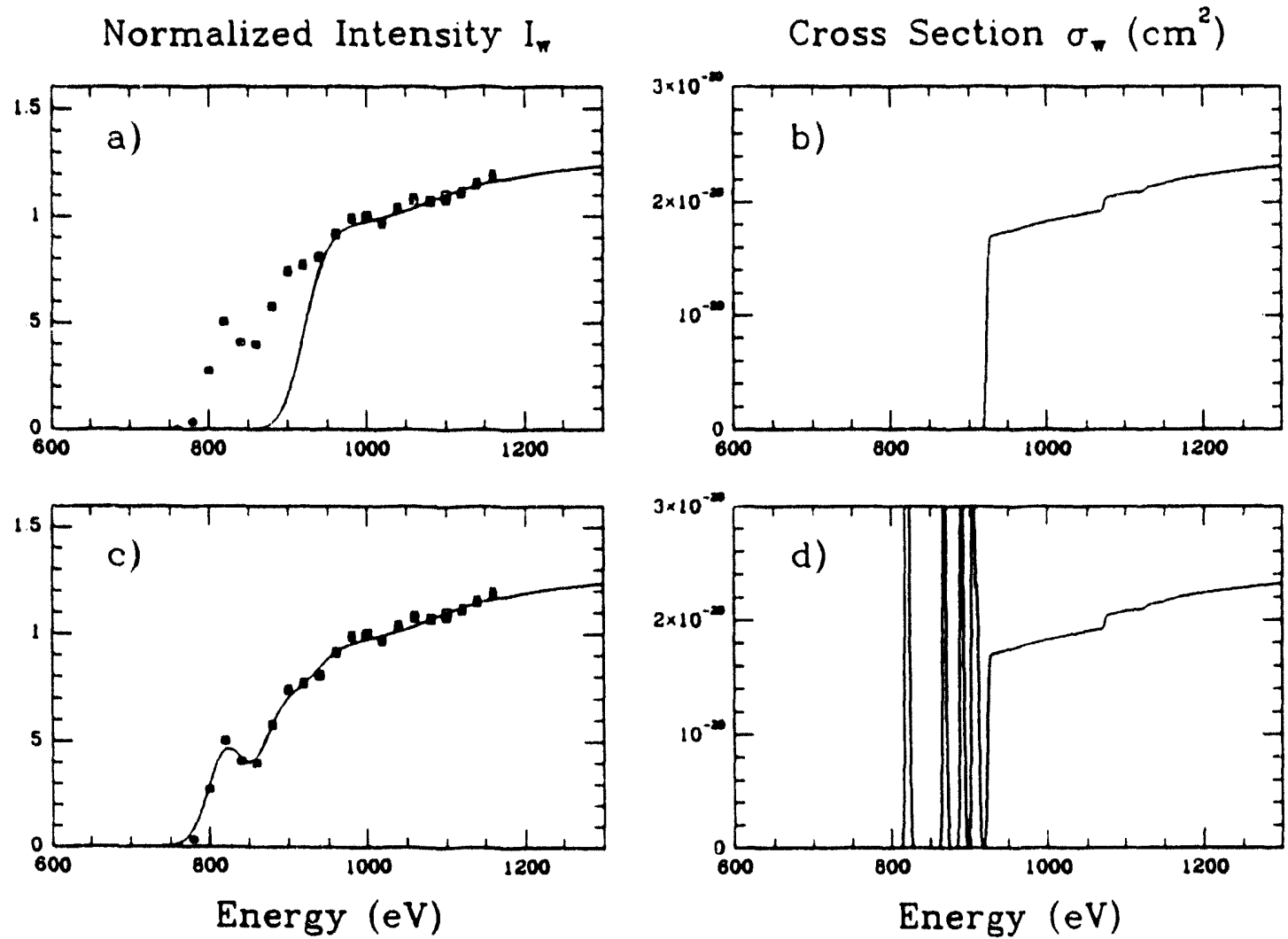

Figure 8.5: Experimental and theoretical cross sections for line $w$. Theoretical cross sections on the right are convolved with a 75-eV FWHM Gaussian function and compared with experimental data on the left. Top panels show cross section derived from calculations by Reed [72], as described in Chapter 6. Bottom panels include dielectronic recombination. 

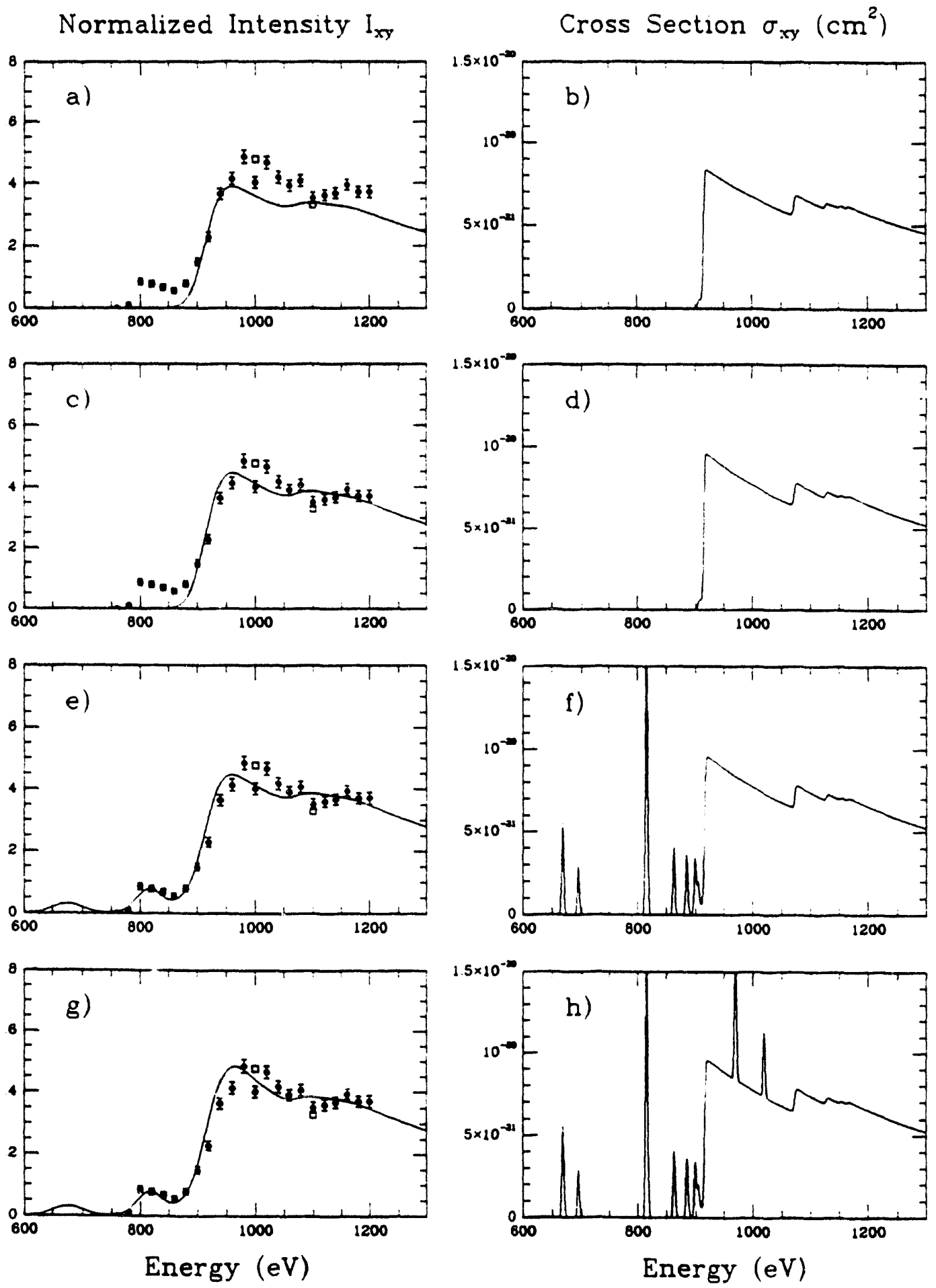

Figure 8.6: Cross sections for $x y$. Panels $a$ and $b$ show theoretical EIE cross section. Panels $c$ and $d$ show cross section scaled up by $15 \%$. Panels $e$ and $f$ include DR, $g$ and $h$ include the $K M M$ and $K M N$ resonances. 

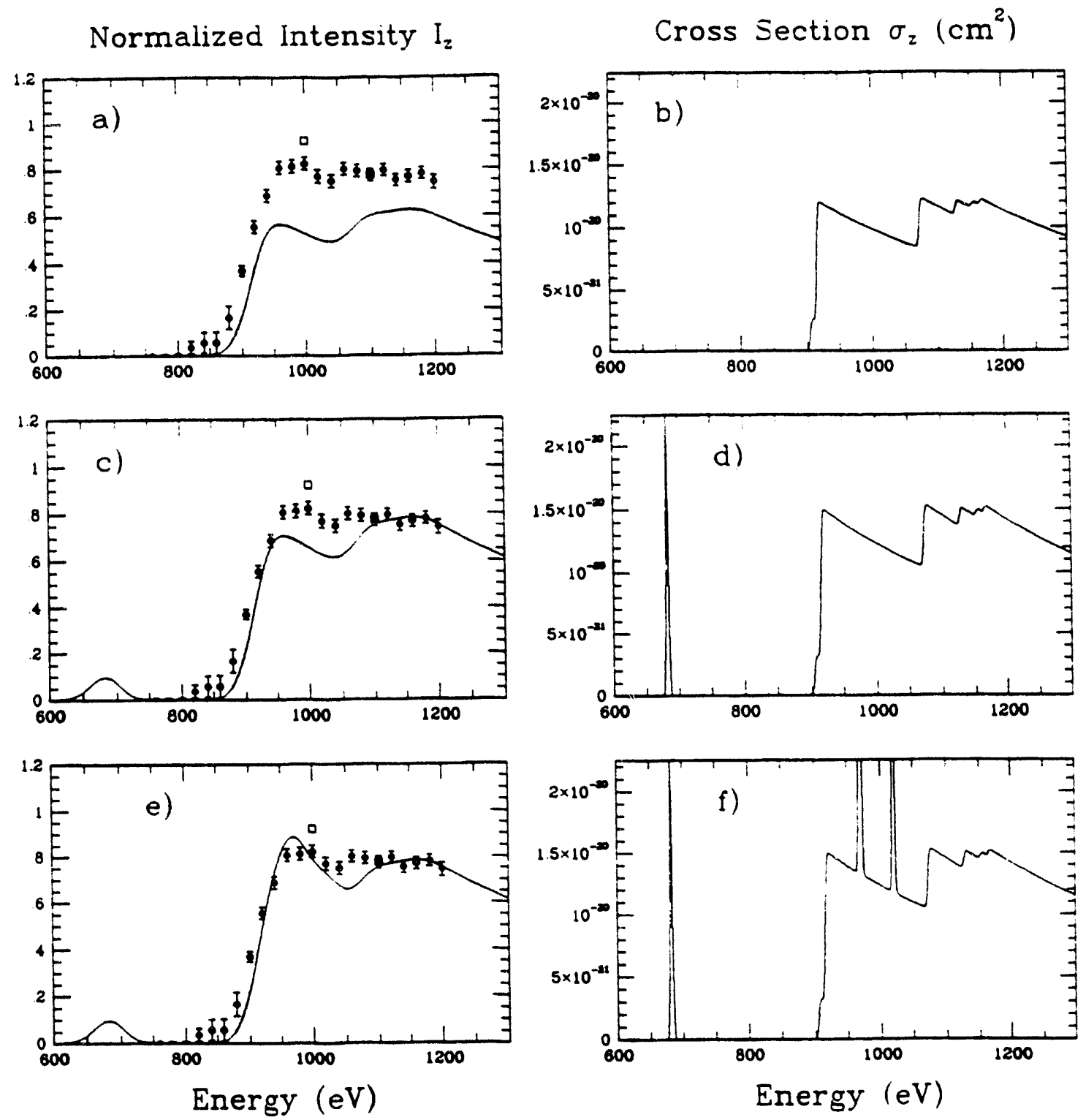

Figure 8.7: Cross sections for $z$. The theoretical EIE cross section is scaled up by $25 \%$ in panels $c$ and $d$. 

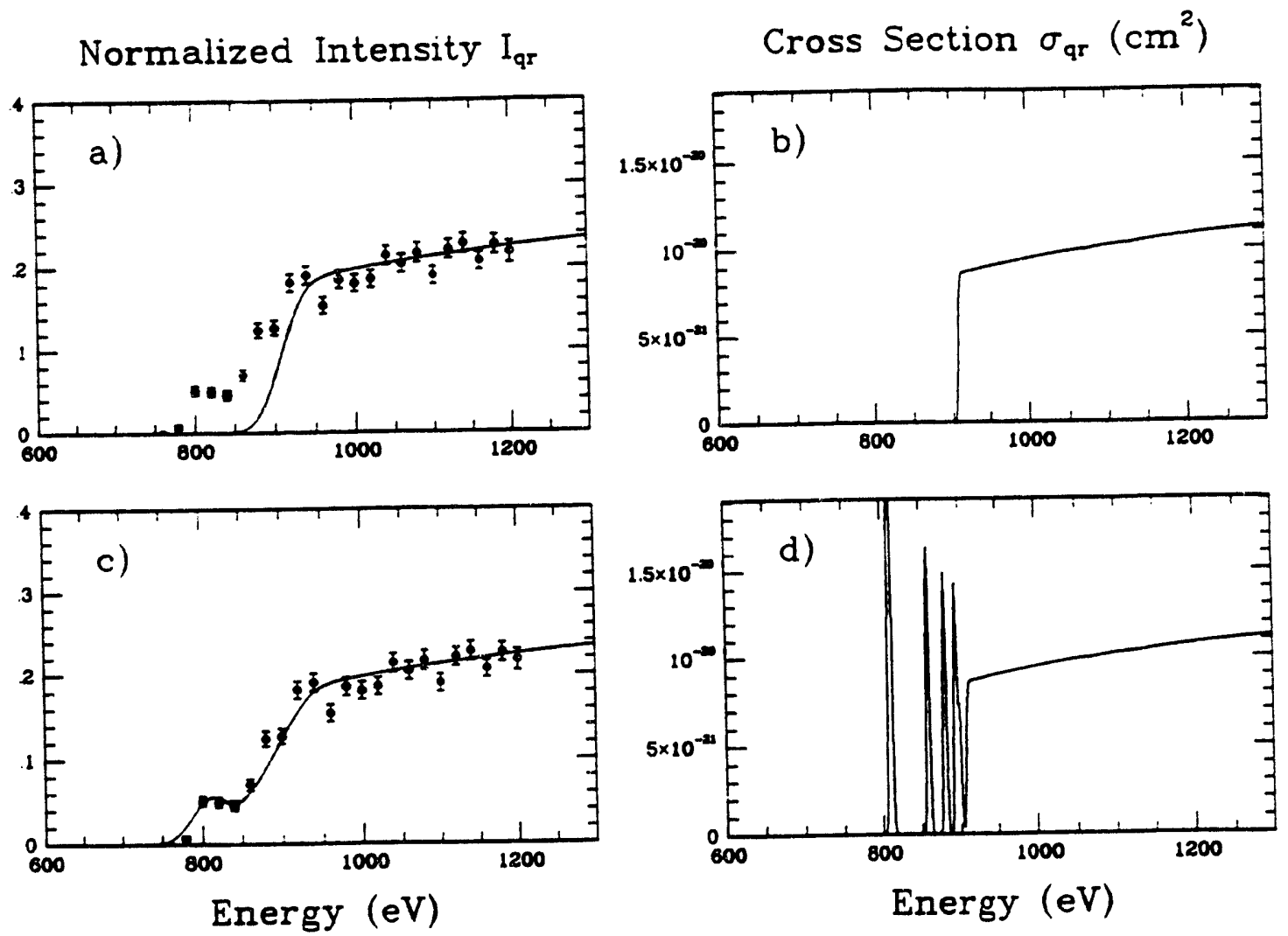

Figure 8.8: Cross sections for $q r$. 
Variations in trap conditions are difficult to estimate, but given the relatively small range of energies covered and the maintenance of a (presumed) constant electron density, $n_{e} n_{i o n}$ should be constant at the level of a few percent. Net systematic errors are therefore dominated by polarization uncertainties, and range from about $5 \%$ for $z$ to $10 \%$ for $x y$. Statistical uncertainties are largest for the weakest line, $x y$, for which $5 \%$ is typical at energies above threshold.

\subsubsection{Determination of Cross Sections and Resonance Strengths}

For each emission line, several graphs are presented, arranged in pairs of experimental data and theoretical cross sections. The plots on the right show the theoretical cross section for that line (or blend). The topmost plot, labeled as $b$, shows cross sections based on calculations by Reed that include the effects of cascades and collisional transfer at the nominal electron density of $5.7 \times 10^{11} \mathrm{~cm}^{-3}$, as described in Chapter 6 . For $q r$, the fluorescence yields calculated by Vainshtein and Safronova are used to derive the theoretical line excitation cross section shown (see equation 8.5). Subsequent plots on the right side show the cross section as it is rescaled, as DR resonances are added, and as resonant excitation (RE) resonances are included. Each of these steps will be explained shortly. KLL DR resonances, previously measured in section 8.1, are included for completeness. The p.' ts on the left show the experimental data along with the theoretical cross section after being convolved with a 75-eV FWHM Gaussian function to model the effect of energy spread in the electron beam. This beam energy spread is somewhat larger than the expected $50-\mathrm{eV}$ width, but it better matches the data, particularly the widths of the KLM resonances. The plots on the right show the theoretical cross sections convolved with a 6-eV FWHM Gaussian to better display the resonances. 
Note that the derived cross sections and resonance strengths are only relative measurements, just as for the $K L L \mathrm{DR}$ resonance strengths derived in section 8.1. For processes whose rates are proportional to $n_{H e}$, cross sections are relative to $\sigma_{w}$ at 1000 $\mathrm{eV}$, is taken to be $\sigma_{w}=1.828 \times 10^{-20} \mathrm{~cm}^{2}$. For processes whose rates are proportional to $n_{L i}$ (namely emission from $q r$ and its DR satellites), cross sections are relative to $\sigma_{q r}$ at $1000 \mathrm{eV}$, taken to be $9.48 \times 10^{-21} \mathrm{~cm}^{2}$, based on excitation cross section calculations by Reed and assuming that the fluorescence yield of $q$ is 0.637 (see Eq. 8.5).

The procedure for determining relative cross sections and resonance strengths for each line begins with rescaling the effective excitation cross section (based on calculations by Reed [72], as described in Chapter 6) so that it matches (by eye) the experimental data at energies between about 1100 and $1200 \mathrm{eV}$. This requires that the initial values of $\sigma_{x y}$ and $\sigma_{z}$ be multiplied by factors of 1.15 and 1.25 , respectively.

Then DR satellites, from $K L M(n=3)$ to $K L Q(n=7)$, were added below threshold. Estimated resonance energies, shown in Table 8.3, were extrapolated from calculations by Chen [22] for $K L L$ and $K L M$ Li-like satellites, and Chen [23] for $K L L$ Be-like satellites. The resonance strengths of the $w$ satellites were determined by adding 1-eV-wide peaks at each resonance energy in the theoretical cross section, and simply adjusting their heights until the convolved cross section matched the experimental data. This is essentially the same process described in section 8.1.3 used to find the strengths of $K L L$ resonances, although the strengths of individual resonances become less reliable as the resonances get closer to the EIE threshold because they cannot be resolved in energy. The strengths of the $w$ resonances were then scaled (all together) to match the $x y$ and $q$ data. The resulting fits were quite good, with the exception of the $K L M$ resonance of $x y$ which had to be significantly increased to match the data. Results are shown in Table 8.3. 
Table 8.3: High order resonances. Resonance energies (in $\mathrm{eV}$ ) are estimated from results of Chen [22] for DR resonances and from Ref. [70] for RE resonances. Resonance strengths (in $10^{-19} \mathrm{~cm}^{2} \mathrm{eV}$ ) are derived from experimentally observed cross sections, as described in the text.

\begin{tabular}{lcccccccc}
\hline \hline & \multicolumn{2}{c}{$w$} & \multicolumn{2}{c}{$x y$} & \multicolumn{2}{c}{$q r$} & \multicolumn{2}{c}{$z$} \\
Resonance & $E_{\text {res }}$ & $S$ & $E_{\text {res }}$ & $S$ & $E_{\text {res }}$ & $S$ & $E_{\text {res }}$ & $S$ \\
\hline $\mathrm{KLM}$ & 820 & 4.60 & 815 & 0.80 & 810 & 1.53 & $\ldots$ & $\ldots$ \\
$\mathrm{KLN}$ & 868 & 2.40 & 863 & 0.18 & 858 & 0.80 & $\ldots$ & $\ldots$ \\
$\mathrm{KLO}$ & 890 & 2.20 & 885 & 0.16 & 880 & 0.73 & $\ldots$ & $\ldots$ \\
$\mathrm{KLP}$ & 904 & 2.00 & 899 & 0.15 & 894 & 0.67 & $\ldots$ & $\ldots$ \\
$\mathrm{KLQ}^{+}$ & 909 & 1.00 & 904 & 0.08 & 899 & 0.33 & $\ldots$ & $\ldots$ \\
& & & & & & & & \\
$\mathrm{KMM}$ & $\ldots$ & $\ldots$ & 970 & 0.50 & $\ldots$ & $\ldots$ & 970 & 2.00 \\
$\mathrm{KMN}^{+}$ & $\ldots$ & $\ldots$ & 1020 & 0.25 & $\ldots$ & $\ldots$ & 1020 & 1.00 \\
\hline \hline
\end{tabular}


Since the $K L M$ resonances are nearly resolved in energy from higher resonances. their strengths should be nearly as accurate as those for the $K L L$ satellites. In fact. excellent agreement is found between theory and experiment for $w$ 's $K L M$ and $K L N$ satellites. Although deciding which transitions are satellites of $w$ and which are satellites of $x y$ was somewhat uncertain because of the limited accuracy of their theoretical wavelength predictions, Nilsen [65] and Chen [21] predict that $w$ 's $K L M$ satellites have a total strength of $4.68 \times 10^{-19}$ and $3.86 \times 10^{-19} \mathrm{~cm}^{2} \mathrm{eV}$, respectively, compared to the experimental value of $4.6 \times 10^{-19}$ (estimated accuracy 20\%). Nilsen also predicts a strength of $2.27 \times 10^{-19} \mathrm{~cm}^{2} \mathrm{eV}$ for $w_{K L N}$, compared to $2.4 \times 10^{-19}$ experimentally. Agreement is not as good for $y_{K L M}$, measured as $8.0 \times 10^{-20} \mathrm{~cm}^{2} \mathrm{eV}$ $( \pm 25 \%)$ and predicted at $3.5 \times 10^{-20}$ by Nilsen and $3.0 \times 10^{-20}$ by Chen. Although individual DR resonances ( $K L N$ and higher) for a given line could not be resolved, the total strength of the $K L N-K L \infty$ DR resonances has an estimated accuracy of $\pm 25 \%$ for each of lines $w, x y$, and $q r$.

After the effects of DR were included, the cross sections for $w$ and $q r$ were well determined, but the experimental cross section for $z$, and to a lesser extent $x y$, was significantly higher than theory for energies above but near the EIE threshold. The explanation for this discrepancy is the omission of resonant excitation peaks from the theoretical cross section (see Chapter 2 for an explanation of RE). The strengths of KMM RE peaks have been calculated by Pradhan, Norcross, and Hummer [70] for neon and several other elements, but the results were folded into calculations of collision rates as functions of temperature without being listed separately, and so are effectively unavailable. Some idea of the importance of RE, however, can be inferred from the work of Chantrenne et al. [19], who measured EIE cross sections near threshold for $w, x, y$, and $z$ in He-like titanium $(Z=22)$ and found that the $K M M$ and $K M N$ resonances increased the integrated cross section for excitation of $z$ by roughly 
$20 \%$ between threshold and the onset of $n=3$ cascades. The resonances were relatively weaker for $x, y$, and $w$. Higher order resonances, such as $K N N$, were considered negligible.

For the neon data presented here, the strengths of the $K M M$ and $K M N$ resonances in $x y$ and $z$ were estimated in the same fashion as for the DR resonances. $E_{r e s}$ for the $K M M$ resonance (actually composed of several related resonances spread over an energy range of $20 \mathrm{eV}$ ) was set at $970 \mathrm{eV}$, in accordance with the calculations of Pradhan et al. [70]. The KMN resonance was set at $1020 \mathrm{eV}$, slightly higher than its true energy to approximate the contributions of higher $K M x$ resonances. The resonance strengths which gave the best fits to the data are listed in Table 8.3. Note, however, that the polarizition of the resonances was not considered (most likely a small effect) and the model used was not very detailed. In addition, no effort was made to fit RE resonances to the $w$ or $q r$ data, although they must exist at some (evidently low) level.

Even after the inclusion of these resonances, a noticeable difference between data and theory still exists just below the onset of $n=3$ cascades in the cross section for $z$, and to a lesser extent in the cross section for $x y$. This was also noted in the results of Chantrenne et al. [19], who observed a "shelf in the triplet line data" just below the cascade jump which they suggested was due to stronger than expected $K M n(n>4)$ resonances.

One other feature that deserves mention is the anomalously large intensity of $z$ relative to $w$ for the $1000-\mathrm{eV}$ steady-state polarization measurement. This measurement had excellent statistics, and all other $1000-\mathrm{eV}$ measurements (from the normalization of high energy data) also showed a larger value of $I_{z}$ than did the Event Mode data. Considering the proximity of the $K M M$ and $K M N$ resonances, the most likely explanation is that the beam energy spread was smaller during steady-state 
measurements (the high energy normalization count as steady-state because the beam energy was held at $1 \mathrm{keV}$ for 1 or $2 \mathrm{msec}$ ) than for the Event Mode measurements which continuously varied the beam energy, thus preventing the beam from "settling down." A narrower beam energy spread would thus make the RE peaks narrower, and increase their relative intensity. This explanation only works, however, if the beam energy was sufficiently close to a resonance. Since the uncertainty in the centroid of the beam energy is 10 to $20 \mathrm{eV}$ for this measurement, it is quite possible that the presumed $1000-\mathrm{eV}$ measurement was actually a bit higher or lower and therefore near either the $K M M$ or $K M N$ resonance.

\subsection{High Energy Data}

At energies above the ionization threshold for He-like (to form $\mathrm{H}$-like) ions, the excitation cross sections for all the $K_{\alpha}$ lines are smoothly varying without any resonances. A few measurements at widely spaced energies therefore suffice to map out the behavior of the cross section as a function of energy. Briefly summarizing the experimental procedure described in Chapter 5 , measurements were made at nine energies between 1.5 and $13 \mathrm{keV}$ (over 14 times the EIE threshold energy). At least one spectrum at each of those energies was obtained by switching the beam energy up and down so that line intensities could be normalized to those at $1 \mathrm{keV}$. The other spectra were collected while the beam energy was held fixed but the ratio of He-like and Li-like ions was varied from one measurement to another. As will be explained, these measurements permitted the determination of relative cross sections as a function of energy for the following: (1) excitation of $w, x y$, and $z$; (2) collisional transfer from ${ }^{3} S_{1}$ to ${ }^{3} P$; (3) innershell ionization of Li-like neon, which excites line $z$. 


\subsubsection{Excitation of $\mathrm{K}_{\alpha}$ Lines at High Energies}

At energies above the ionization threshold of He-like neon and in the low density limit, line intensities are given by

$$
\begin{gathered}
I_{w}=n_{H e} n_{e} \nu_{e} \sigma_{w}+n_{H}\left(n_{e} v_{e} \sigma_{w}^{R R}+n_{0} v_{0} \sigma_{w}^{C E R}\right) \\
I_{x y}=n_{H e} n_{e} \nu_{e} \sigma_{x y}+n_{H}\left(n_{e} v_{e} \sigma_{x y}^{R R}+n_{0} v_{H} \sigma_{x y}^{C E R}\right) \\
I_{z}=n_{H e} n_{e} \nu_{e} \sigma_{z}+n_{H}\left(n_{e} \nu_{e} \sigma_{z}^{R R}+n_{0} v_{0} \sigma_{z}^{C E R}\right)+n_{L i} n_{e} v_{e} \sigma_{z}^{I S I} \\
I_{q r}=n_{L} n_{e} \nu_{e} \sigma_{q r}
\end{gathered}
$$

where $\sigma_{l}^{R R}$ and $\sigma_{l}^{C E R}$ are, respectively, the cross sections for radiative and charge exchange recombination of $\mathrm{H}$-like neon that result in the emission of line $l$. no is the

velocity of neutral neon ions and $v_{H}$ is the velocity of $H$-like ions in the trap. $\sigma_{Z}^{I S I}$ is the cross section for production of line $z$ by inner-shell ionization of a Li-like ion. Other symbols should be familiar.

The total cross section for RR at $1000 \mathrm{eV}$ is approximately 1000 times smaller than the EIE cross sections for excitation of $w, x y$, and $z$. RR is thus unimportant unless $n_{H}$ " $n_{H e}$. Although the H-like line Lyman- $\alpha$ could not be simultaneously observed to provide an indication of the relative abundance of He-like and $\mathrm{H}$-like ions, a computer model of the ionization balance in the EBIT trap showed that $n_{H} / n_{H e}$ never exceeded a value of about 4 for any of the measurements discussed here and was usually less than 1. A few spectra were obtained that did show Ly- $\alpha$ and $w$ simultaneously; the relative strength of these two lines was consistent with model predictions. It was therefore assumed that the radiative recombination of $\mathrm{H}$-like ions had a completely negligible impact on the observed intensities of all $K_{\alpha}$ lines.

The effect of charge exchange recombination is more difficult to estimate. CER cross sections are rather difficult to calculate, and most results are for CER between 
neutral and ionized hydrogen, for which $\sigma C E R \approx 10^{-14} \mathrm{~cm}^{2}[3]$. Cross sections for other elements are roughly the same. CER tends to populate low-angular momentum, high-n states of the recombining ion, which mostly decay directly (or after one cascade) to ground. The cross sections for excitation of the $n=2$ states which produce $K_{\alpha}$ radiation are therefore significantly smaller than the total cross section, probably of order $10^{-16} \mathrm{~cm}^{2}$.

The energy of the neutral ions as they are injected into the trap is that of room temperature, so the relevant collision velocity is that of the ions in the trap. With a well depth of $80 \mathrm{~V}$, the maximum energy for trapped $\mathrm{H}$-like neon ions is $q_{i} V_{\text {well }}=$ $(9 e)(80 \mathrm{~V})=720 \mathrm{eV}$. Assuming an average energy of half that, the velocity of the ions is $6 \times 10^{6} \mathrm{~cm} / \mathrm{sec}$. The pressure of neutral neon gas in the injection chamber was typically a few times $10^{-7}$ torr, corresponding to a density of $10^{10} \mathrm{~cm}^{-3}$. As the gas atoms are ballistically injected into the trap, they tend to spread out, so their density in the trap is probably no higher than $10^{9} \mathrm{~cm}^{-3}$.

The excitation rate $n_{O} v_{H} \sigma^{C E R}$ is then approximately 1 (or less), compared to $n_{e} v_{e} \sigma^{E I E} \approx 100$ at $1000 \mathrm{eV}$. At higher energies, say $10 \mathrm{keV}$, the cross sections for $x y$ and $z$ have dropped by roughly three orders of magnitude from their values at 1000 $\mathrm{eV}$, to approximately $10^{-23} \mathrm{~cm}^{2}$. Since the relative abundance of $\mathrm{H}$-like ions in the trap also increases with energy, CER may begin to compete with EIE for excitation of $x y$ and $z$ at energies of a few or several keV. CER should still be negligible for excitation of $w$ and $q r$ because their EIE cross sections do not decrease as quickly at high energies.

In the following discussions it will be assumed that the recombination of $\mathrm{H}$-like ions does not contribute to the excitation of $K_{\alpha}$ lines. Equations 8.27-8.29 then become

$$
I_{w}=n_{H e} n_{e} \nu_{e} \sigma_{w}
$$




$$
\begin{gathered}
I_{x y}=I_{w} \frac{\sigma_{x y}}{\sigma_{w}} \\
I_{z}=I_{w} \frac{\sigma_{i}}{\sigma_{w}}+I_{q r} \frac{\sigma_{\dot{s}}^{I S l}}{\sigma_{q r}} .
\end{gathered}
$$

These equations are true for the low-density limit. For nonzero densities, they must be modified to include the effect of collisional transfer. To distinguish between values at zero and nonzero densities, let "(0)" after a quantity refer to its value in the lowdensity limit, and " $\left(n_{e}\right)$ " the value at nonzero density $n_{e}$. Lines $w$ and $q r$ are unaffected by collisional transfer so their cross sections and intensities will not be marked with this density notation. If $f_{\text {net }}$ represents the fractional net transfer of " $z$ intensity" to "xy intensity," then the intensities for $x y$ and $z$ can be written as

$$
\begin{gathered}
I_{x y}\left(n_{e}\right)=I_{x y}(0)+I_{z}(0) f_{n e t}\left(n_{e}\right) \\
I_{z}\left(n_{e}\right)=I_{z}(0)\left[1-f_{\text {net }}\left(n_{e}\right)\right] .
\end{gathered}
$$

After a combining with equations 8.32 and 8.33 (which are low-density equations) and a little rearranging we finally obtain

$$
\begin{gathered}
\frac{I_{x y}\left(n_{e}\right)}{I_{w}}=\left[\frac{\sigma_{x y}(0)}{\sigma_{w}}\right]+\left[\frac{f_{n e t}}{1-f_{n e t}}\right] \frac{I_{j}\left(n_{e}\right)}{I_{w}} \\
\frac{I_{z}\left(n_{e}\right)}{I_{w}}=\left[\left(1-f_{n e t}\right) \frac{\sigma_{i}(0)}{\sigma_{w}}\right]+\left[\left(1-f_{n e t}\right) \frac{\sigma_{i}^{I S I}}{\sigma_{q r}}\right] \frac{I_{q r}}{I_{w}}
\end{gathered}
$$

All the intensity ratios in these two equations are measurable quantities. Note also the linear relationships between those ratios. If $b_{x y}$ and $m_{x y}$ are defined as, respectively, the vertical axis intercept and slope of the line that results when $I_{x y}\left(n_{e}\right) / \pi_{w}$ is plotted versus $I_{z}\left(n_{e}\right) I_{w}$, and $b_{z}$ and $m_{z}$ are the intercept and slope of the line relating $I_{z}\left(n_{e}\right) / I_{w}$ and $I_{q r}\left(n_{e}\right) I_{w}$, then

$$
\frac{\sigma_{x y}(0)}{\sigma_{w}}=b_{x y}
$$




$$
\begin{aligned}
\frac{\sigma_{i}(0)}{\sigma_{w}} & =b_{z}\left(1+m_{x y}\right) \\
\frac{\sigma_{z}^{I S I}(0)}{\sigma_{w}} & =m_{z}\left(1+m_{x y}\right) \\
f_{n e t} & =\frac{m_{x y}}{1+m_{x y}} .
\end{aligned}
$$

The product of electron density and the cross section for excitation from ${ }^{3} S_{1}$ to ${ }^{3} P_{3,1,0}$, $n_{e} \sigma\left({ }^{3} S_{1} \rightarrow^{3} P\right)$, can also be derived, from the value of $f_{n e t}$. If $f$ is the fraction of ${ }^{3} S_{1}$ states that are excited to ${ }^{3} P$ rather than that decay to ground, and $1 / 3$ of those ${ }^{3} P$ states then decay to produce $x y$ emission and the other $2 / 3$ decay back to ${ }^{3} S_{1}$ where the process is repeated ad infinitum (an approximation accurate to $1 \%$ ), then by summing the resulting geometric series we obtain

$$
f=\frac{3 f_{\text {net }}}{1+2 f_{\text {net }}}
$$

Using the atomic model discussed in Chapter $6, f$ is also given by

$$
f=\frac{\left.1.07 n_{e} \nu_{e} \sigma{ }^{3} S_{1} \rightarrow{ }^{3} P_{2,1.0}\right)}{1.07 n_{e} \nu_{e} \sigma\left({ }^{3} S_{1} \rightarrow{ }^{3} P_{2,1.0}\right)+A_{r}^{2}}
$$

where $A_{r} z$ is the rate for radiative decay of the ${ }^{3} S_{1}$ state, equal to $1.10 \times 10^{4} \mathrm{sec}^{-1}$. This can be rewritten to finally obtain

$$
n_{e} \sigma\left({ }^{3} S_{1} \rightarrow{ }^{3} P_{2,1,0}\right)=\frac{A_{r}^{z}}{v_{e}}\left(\frac{f}{1-f}\right) .
$$

\subsubsection{Data Analysis and Fit Results}

Line intensities and their uncertainties were derived in the usual way using the PKFIT program, and then corrected for polarization using equations $6.41-6.44$. The 
polarizations of lines $x y$ and $z$, however, depend upon how much of their respective intensities comes from EIE. ISI, or collisional transfer. Polarizations from EIE were computed as described in Chapter 6. Since ISI of Li-like ions removes the 1 s electron involved in the collision, the remaining electrons have no "knowledge" of the incident electron's direction, and so $z$ emission as a result of ISI is unpolarized. According to the model described in Chapter 6, $P_{x y}=P_{z}+0.177$ as a result of collisional transfer. With $P_{z}^{E I E}, P_{z}^{I S I}, P_{x y}^{E I E}$, and $P_{x y}^{I S I}$ thus determined, only the relative intensities of each contribution are necessary to determine the average, weighted polarizations of $x y$ and z. This was done using the following equations:

$$
\begin{gathered}
I_{z}^{E I E}=I_{w} \frac{\sigma_{z}(0)}{\sigma_{w}}\left(1-f_{n e t}\right)=I_{w} b_{z} \\
I_{z}^{I S I}=I_{q r} \frac{\sigma_{i}^{I S I}(0)}{\sigma_{q r}}\left(1-f_{n e t}\right)=I_{q r} m_{z} \\
I_{x y}^{E I E}=I_{w} \frac{\sigma_{x y}(0)}{\sigma_{w}}+I_{w} \frac{\sigma_{z}(0)}{\sigma_{w}} f_{\text {net }}=I_{w}\left(b_{x y}+b_{z} m_{x y}\right) \\
I_{x y}^{I S I}=I_{q r} \frac{\sigma_{z}^{I S I}(0)}{\sigma_{q r}} f_{n e t}=I_{q r} m_{z} m_{x y} .
\end{gathered}
$$

The values of $b$ and $m$, however, are results from fitting the corrected intensity data. which depend on the net line polarizations, which in turn depend on the relative contributions of EIE and ISI. Data analysis is thus an iterative process. For clarity, the following description of the fitting process will assume that the final corrected intensities are used.

The intensity ratio data is plotted in Figures 8.9 and 8.10. The straight lines in each plot are the results of a multivariate fitting procedure based on Deming's method [57]. The multivariate method, rather than a simple weirhted linear least-squares fitting routine, was required because there are actually three variables, each with its own uncertainties, involved in each fit: $I_{z}, I_{q r}$, and $I_{w}$ in Figure 8.9; and $I_{x y}, I_{z}$, and $I_{w}$ 

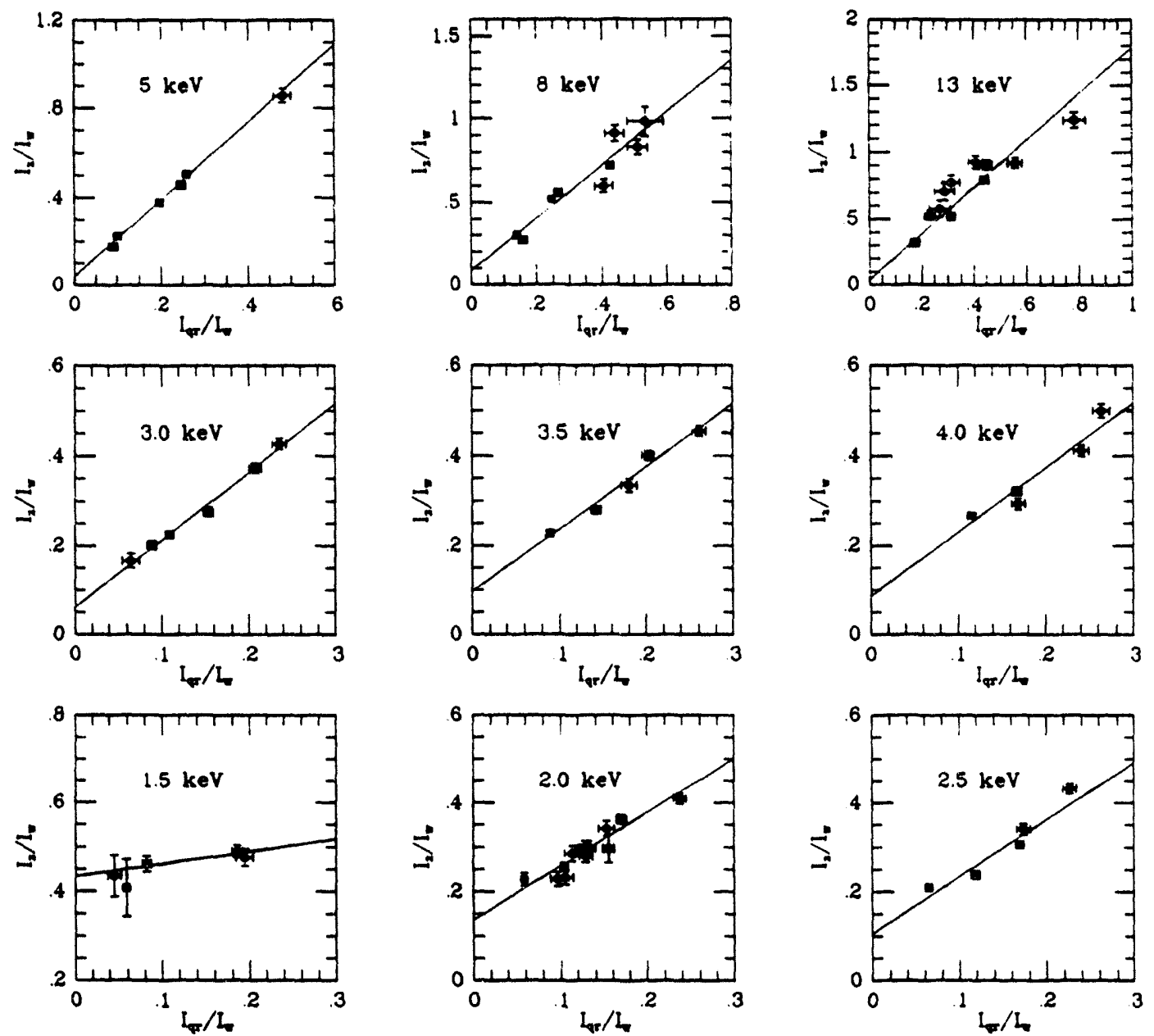

Figure 8.9: High energy data for lines $z$ and $q r$. Each panel has the same aspect ratio so the slopes of linear fits can be compared. Note how the y-intercepts (corresponding to EIE of $z$ ) decrease with energy and the slopes (corresponding to ISI excitation of $z$ ) increase with energy. 

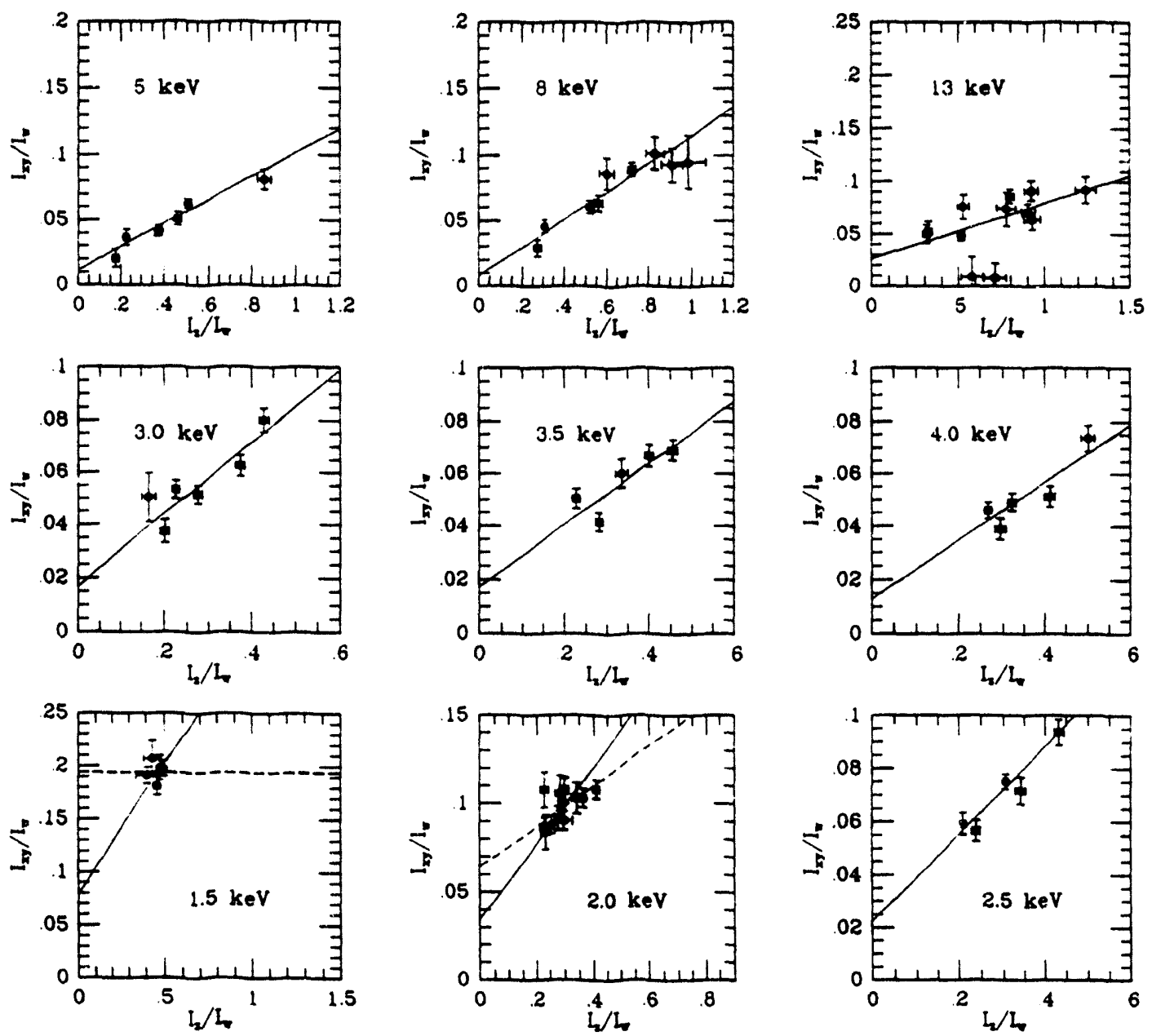

Figure 8.10: High energy data for $x y$ and $z$. Each panel has the same aspect ratio. Note that $y$-intercepts (corresponding to EIE of $x y$ ) and slopes (corresponding to collisional transfer from $z$ to $x y$ ) generally decrease with increasing energy. Fits for 1.5 and $2.0 \mathrm{keV}$ data fixed the $y$-intercept at values predicted by theory. Dashed line fits correspond to open circle data points in Figure 8.11, and are used for comparisons with cross sections derived from solid line fits. 
in Figure 8.10. The actual fitting program was written by David Vogel and provides estimates of the uncertainties of the two fit parameters.

As can be seen in the Figures, the quality of the fits was generally quite good, although the statistical quality of the $I_{x y} / I_{w}$ vs. $I_{z} / I_{w}$ data is noticeably poorer than that in Figure 8.9 because of the small intensity of $x y$ at high energies. In this regime, the EIE cross section for $x y$ is extremely small and virtually all of $I_{x y}$ comes from collisional transfer from line $z$.

The 1.5 and $2.0 \mathrm{keV}$ data in Figure 8.10 had to be specially treated because the relative contribution of ISI to the intensities of $x y$ and $z$ could not be varied over a large enough range to give good fits. For the $1.5 \mathrm{keV}$ data, the dashed line marks the average value of $I_{x y} / I_{w}$ as a sort of worst-case scenario assuming that there is no collisional transfer from $z$. The dashed line for the $2 \mathrm{keV}$ data is the fitting program result, but from inspection it is clear that a wide range of slopes would give adequate fits. Instead, the y-intercepts were set at values derived from the theoretical cross sections computed by Reed [72] and the slopes determined from best-fit lines drawn by eye.

Fit results at each energy were then inserted into equations $8.45-8.48$. For equation 8.48 , a density of $5.7 \times 10^{11} \mathrm{~cm}^{-3}$ was assumed. The experimental collisional cross section $\sigma\left({ }^{3} S_{1} \rightarrow{ }^{3} P_{2,1,0}\right)$ was then divided by 3 (since excitation to ${ }^{3} P_{2,1,0}$ has the statistical proportions 5:3:1) to give $\sigma\left({ }^{3} S_{1} \rightarrow{ }^{3} P_{1}\right)$. Results are shown in Figure 8.11, along with theoretical predictions marked by solid curves.

Filled circle data points represent results derived from the solid line fits in Figures 8.9 and 8.10 . Open circles at 1.5 and $2.0 \mathrm{keV}$ denote results using the dashed line fits in Figure 8.9. Error bars were determined using standard error propagation techniques. In Figures $8.11 a$ and $8.11 \mathrm{c}$, data points at 1.5 and $2.0 \mathrm{keV}$ do not have error bars, but the difference between solid and open data points gives an indication of 
relative uncertainties. Note that if the assumed values of $\sigma_{x y}(0) / \sigma_{w}$ at 1.5 and $2.0 \mathrm{keV}$ were increased by $15 \%$ over their theoretical values, in accordance with the lowenergy results of section 8.2, then the corresponding values of $\left.\sigma^{3} S_{1} \rightarrow{ }^{3} P_{1}\right)$ in Figure $a$ would be even closer to the solid line marking theoretical predictions. In Figures $b$ and $d$, error bars were computed from the uncertainties in $b_{z}$ and $m_{z}$, with no error contributions from $m_{x y}$.

The theoretical collisional transfer cross section in Figure $a$ is that used in the theoretical polarization calculations of Chapter 6 . It is normalized to $\sigma\left({ }^{3} S_{1} \rightarrow{ }^{3} P_{1}\right)=$ $2.46 \times 10^{-18} \mathrm{~cm}^{2}$ at $750 \mathrm{eV}$, as calculated by Sampson and Zhang [74], and assumes that $\sigma$ scales as $E^{-0.84}$. As can be seen, the data are in good agreement with the predicted energy dependence.

Figure $b$ also shows excellent agreement with theory, with some qualifications. The theoretical cross section for ISI excitation of $z$ was computed using the semiempirical Lotz formula [56], assuming that $3 / 4$ of the innershell ionizations of Li-like Ne result in a $1 s 2 s^{3} S_{1}$ state. The other $1 / 4$ produce a $1 s 2 s^{1} S_{0}$ state. The general Lotz formula is

$$
\sigma(E)=\sum_{j=1}^{N} a_{j} q_{j} \frac{\ln \left(E / I_{j}\right)}{E I_{j}}\left[1-b_{j} e^{-c_{j}\left(E / I_{j}-1\right)}\right]
$$

where the index $j$ denotes the subshell, $l_{j}$ is the ionization potential of the subshell, and $q_{j}$ is the occupation number. The constants $a_{j}, b_{j}$, and $c_{j}$ are parameters determined from experimental data. Since no experimental data are available for Li-like neon, the default values of $a=4.5 \times 10^{-14} \mathrm{~cm}^{2} \mathrm{eV}^{2}, b=0$, and $c=0$ are used here. For removal of a $1 s$ electron from Li-like neon, $q_{j}=2$ and $I_{j}=1107 \mathrm{eV}$. The cross section for ISI excitation of $z$ is thus

$$
\sigma_{z}^{d S I}(E)=\frac{3}{4}\left(4.5 \times 10^{-14} \mathrm{~cm}^{2} \mathrm{eV}^{2}\right) 2 \frac{\ln (E / 1107 \mathrm{eV})}{E(1107 \mathrm{eV})} .
$$



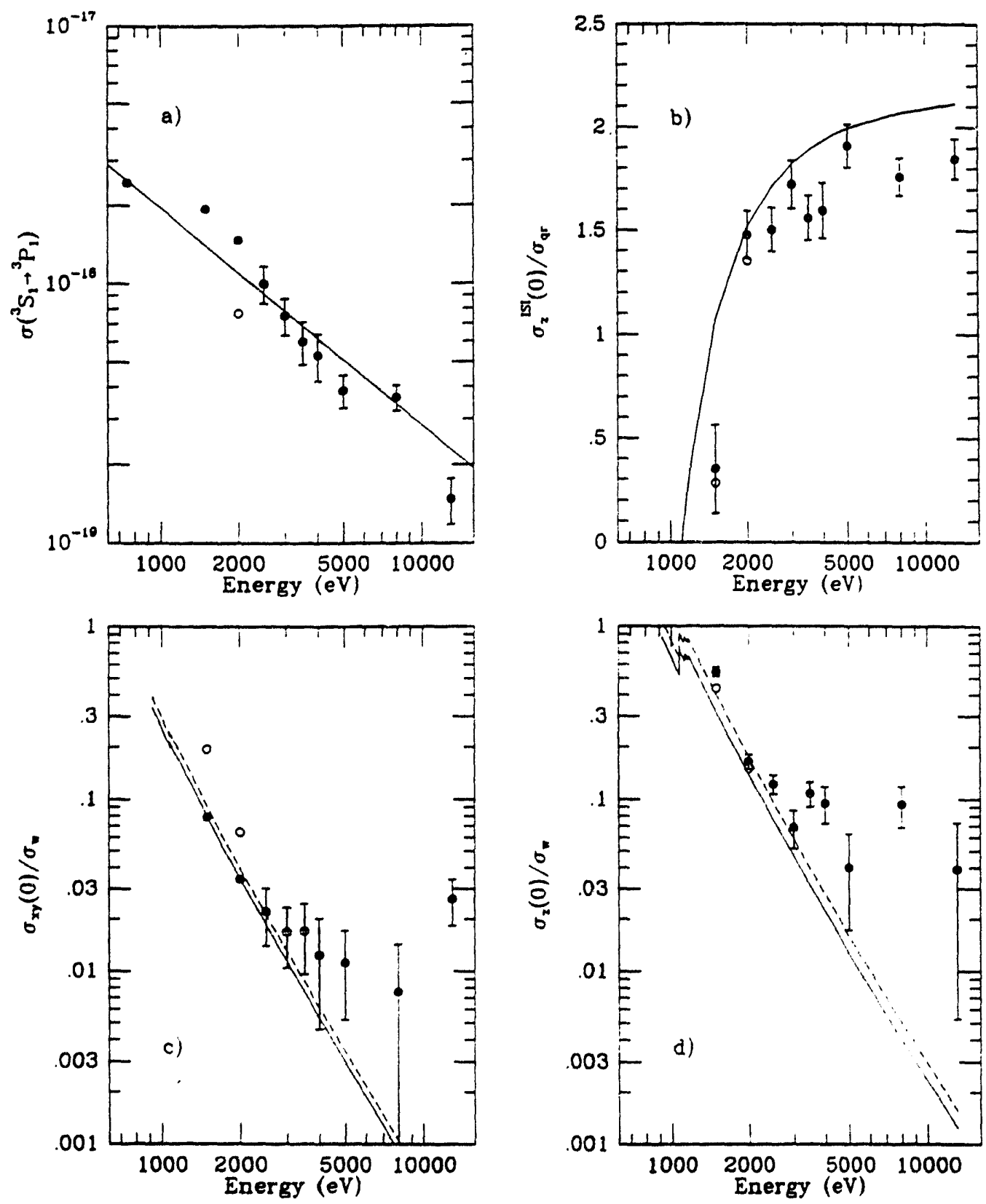

Figure 8.11: Relative cross sections derived from high energy data. Open circle data points are computed using dashed line fits from Figure.8.10. Theoretical cross sections are based on calculations by Sampson and Zhang [74] for panel $a$ and Reed [72] for panels $b, c$, and $d$. Fluorescence factor for $q$ computed by Vainshtein and Safronova was used in calculation of theoretical value of $\sigma_{q r}$ (panel $b$ ), and the Lotz formula was used for $\sigma_{z} I S I$. Effects of CER are seen at highest energies in panels $c$ and $d$. The dashed lines in panels $c$ and $d$ are the theoretical cross sections for $x y$ and $z$ scaled up by $15 \%$ and $25 \%$, respectively (to agree with the low-energy results). 
From comparisons with distorted-wave and other atomic model calculations, as well as experimental data, the above formula has an estimated error of approximately $\pm 20 \%$.

The cross section for excitation of $q r$ is based on the calculations of Reed [72], which have an estimated accuracy of $\pm 20 \%$. As discussed in section 8.1, those calculations are for the excitation of the states that decay to produce $q$ and $r$. To obtain the effective cross section for emission of the $q r$ blend, the fluorescence yield $W$ must also be determined (see equation 8.4). For the theoretical curve shown, the fluorescence yield $W_{q}=0.637$ computed by Vainshtein and Safronova [79] was used. If the results of Chen [21] or Nilsen [65] were used, the theoretical curve would be a factor of 1.82 or 1.23 , respectively, higher. Given the estimated theoretical and experimental uncertainties, Nilsen's prediction for $W_{q}$ is consistent with the data, but Chen's is not.

The theoretical curves in Figures $8.11 c$ and $d$ are based on the results of Reed [72] for EIE of He-like ions in the low density limit. Agreement with the data is generally good up to several keV, particularly if the theoretical cross sections for $x y$ and $z$ are scaled up by $15 \%$ and $25 \%$, respectively, (plotted with dashed lines) based on the low-energy results of section 8.2. At the highest energies, however, the experimental data are significantly above theory. The most likely explanation for these deviations is charge exchange recombination of $\mathrm{H}$-like neon ions.

Although there are methods to minimize the number of $\mathrm{H}$-like ions, such as briefly raising the beam energy above the ionization threshold to collect spectra and then keeping the beam energy low for a long period so the ions could recombine, or dumping the ions from the trap after every high energy probe, the data collection efficiency would be prohibitively low. A less exact but more efficient method would be to monitor the $\mathrm{H}$-like ion abundance while collecting He-like $K_{\alpha}$ spectra and then 
subtract off the CER contribution to each line's intensity. As explained in Chapter 4, however, existing solid state detectors do not have enough energy resolution to separate He-like and H-like emission, and the FCS does not have enough bandwidth to simultaneously observe the H-like Ly- $\alpha$ line and the He-like spectra. The solution would be to have a second high resolution spectrometer, or to switch back and forth between $\mathrm{Ly}-\alpha$ and the He-like $K_{\alpha}$ lines. Even then, the average collisional velocity, density of neutral atoms, and effective CER excitation cross section for each line would have to be estimated (see equations 8.27-8.29). As discussed in section 8.4, however, experimental complications arising from CER at high energies are of very little practical importance because $x y$ and $z$ emission is generally dominated by contributions from ion-electron collisions at low energies.

\subsubsection{Normalization of $\sigma_{w}(E)$}

As explained in Chapter 5, spectra obtained at different energies were normalized to data at $1 \mathrm{keV}$ to determine the energy dependence of each line's excitation cross section. Line $w$ was the basis of this normalization, since its intensity is not affected by the abundance of Li-like ions. The normalization relied on the amount of time spent collecting data at the high and low energies and implicitly assumed that trap conditions, specifically the density and distribution of ions and electrons, did not change as the beam energy was raised and lowered.

The number of counts in line $w$ depends on the amount of time spent collecting data $(t)$, so the integrated intensity is given by

$$
I_{w}=n_{H e} n_{e} \nu_{e} \sigma_{w} t
$$

The electron beam energy is equal to $(1 / 2) m v^{2}$, so the cross section at energy $E$ is 


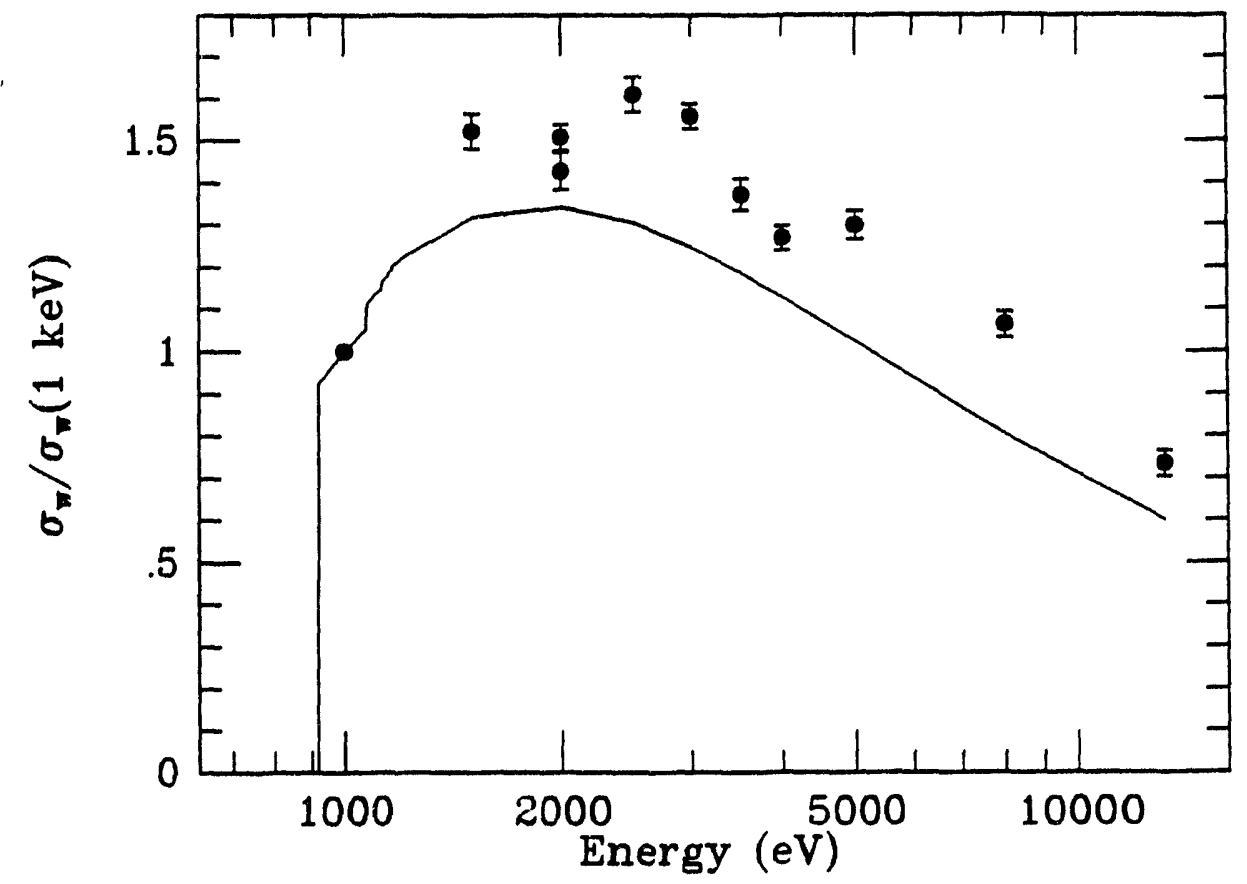

Figure 8.12: Energy dependence of $\sigma_{w}$. Data were normalized to the intensity of $w$ at $1000 \mathrm{eV}$. Solid curve is based on theoretical calculations of Reed [72]. 


$$
\sigma_{w}(E)=\frac{I_{w}(E)}{n_{H e} n_{e} \sqrt{2 E / m_{e}} t(E)}
$$

and the ratio of cross sections at energy $E$ and $1 \mathrm{keV}$ is

$$
\frac{\sigma_{w}(E)}{\sigma_{w}(1)}=\frac{I_{w}(E)}{I_{w}(1)} \sqrt{\frac{1}{E}} \frac{t(E)}{t(1)}
$$

where $E$ is in $\mathrm{keV}$ and $n_{\mathrm{He}} n_{e}$ is assumed constant.

For each normalization measurement--there were two for $2 \mathrm{keV}$--the amount of time spent at each energy was extracted from the Event Mode timing data. Line intensities were determined in the usual way using PKFIT and then corrected for polarization. Results are shown in Figure 8.12.

Apart from the $1 \mathrm{keV}$ normalization point, all the data points lie approximately $20 \%$ above theory. It is not clear if this is a real result or if there is a systematic error in the normalization procedure. If the cause is an instrumental effect, then it is almost certainly because of variations in trap conditions (ion density and species abundances, electron density, ion-beam overlap) between $1 \mathrm{keV}$ and the higher energies. The best method for answering this question would be to simultaneously observe a He-like line whose cross section is accurately known as a function of energy and see if its derived cross section behaved as expected. The obvious candidate is the RR peak as He-like neon recombines to form Li-like ions, but that emission is too weak to be distinguished from background emission. A more practical method would be to use the H-like Ly- $\propto$ line, but it would then be necessary to make sure that the ionization balance in the trap did not change as the energy was varied. 


\subsection{Application to Temperature Diagnostic}

The purpose of all these measurements is to provide benchmarks against which theoretical calculations can be compared, so that plasma diagnostics can be based on reliable data. Ideally, experimental and theoretical results could be compared for individual processes so that specific aspects of theory (such as DR resonance strengths or cascades from EIE of levels with $n>2$ ) that are incorrect could be identified and targeted for study. Most experimental data on X-ray line emission has come from plasma sources such as tokamaks where a continuous range of electron energies are present and the energy distribution can usually be described by one or more temperatures. Astrophysical sources, of couse, are also plasmas. It is therefore not surprising that most published theoretical results have presented line excitation rates in terms of temperature, convolving cross sections with Maxwellian energy distributions. The resulting rate coefficient for a line $l$ is given by

$$
\gamma_{l}(T) \equiv\left\langle v_{e} \sigma_{l}\left(v_{e}\right)\right\rangle=\left(\frac{2}{k T}\right)^{3 / 2} \frac{1}{\sqrt{m_{e} \pi}} \int_{0}^{\infty} E \sigma_{l}(E) e^{-E / k T} d E \quad\left[\mathrm{~cm}^{3} / \mathrm{sec}\right]
$$

where $<>$ means averaged over all velocities according to a Maxwellian distribution. Multiplying by the ion and electron densities then gives the number of emitted photons per second per cubic centimeter.

Convolving a cross section with energy, however, completely conceals all detail contained in the cross section, such as cascade jumps and DR and RE resonances. Nevertheless, to compare with most published results, it is necessary to compute rate coefficients.

One of the most important He-like diagnostics is that for temperature, using the line ratio $G=\left(I_{x y}+I_{z}\right) / I_{w}$ where the intensities are those emitted at a given temperature, that is, the line intensities are proportional to their respective rate coefficients. Using 
the results of section 8.2 for low energy cross sections and scaling up the theoretical cross sections $\sigma_{x y}$ and $\sigma_{z}$ (by $15 \%$ for $x y$ and $25 \%$ for $z$, as indicated by experimental results), the line ratio $G$ was computed as a function of temperature. So that the resulting function could be compared with published results, DR resonances were not included, and contributions from $\mathrm{H}$-like and Li-like ions were excluded. The results are shown in Figure 8.13, along with the predictions of one of the best theoretical models. The experimental curve when including DR is also shown to demonstate the importance of $D R$ at low temperatures.

Agreement between experiment and theory is not bad, with some exceptions. At low temperatures, where RE is most important (contributing roughly $10 \%$ of $I_{z}$ and $5 \%$ of $I_{x y}$ ), Pradhan et al. [70] predict a significantly higher value of $G$. The results of Chantrenne et $a !$. for He-like $\mathrm{Ti}$ [19] showed that theory significantly overestimated the strength of the $K M M$ resonance (the strongest of the RE peaks). It may be that Pradhan et al. also overestimate the importance of RE. In practical terms, however, the disagreement is of relatively minor importance since He-like emission drops off drastically at temperatures below roughly $10^{6} \mathrm{~K}$, and so it is unlikely that $K_{\alpha}$ emission could be observed from astrophysical sources at these low temperatures. At higher temperatures, above a few times $10^{6} \mathrm{~K}$, the predictions of Pradhan et al. fall below measured values. This is probably the result of underestimating the EIE cross sections of $x y$ and $z$.

Because of their rapid decline with energy (scaling as roughly $E^{-3}$ ), the most important parts of the cross sections for $x y$ and $z$ are at relatively low energy, below one or two times the 1196-eV ionization threshold of He-like neon. Over half of $I_{x y}$ and $I_{z}$ comes from energies below $1200 \mathrm{eV}$ at temperatures up to about $7 \times 10^{6} \mathrm{~K}$, and more than $90 \%$ from energies below $2000 \mathrm{eV}$ up to $10^{7} \mathrm{~K}$. In contrast, over half of $I_{w}$ comes from energies above $2000 \mathrm{eV}$ at $10^{7} \mathrm{~K}$, and the fraction continues to rise well 


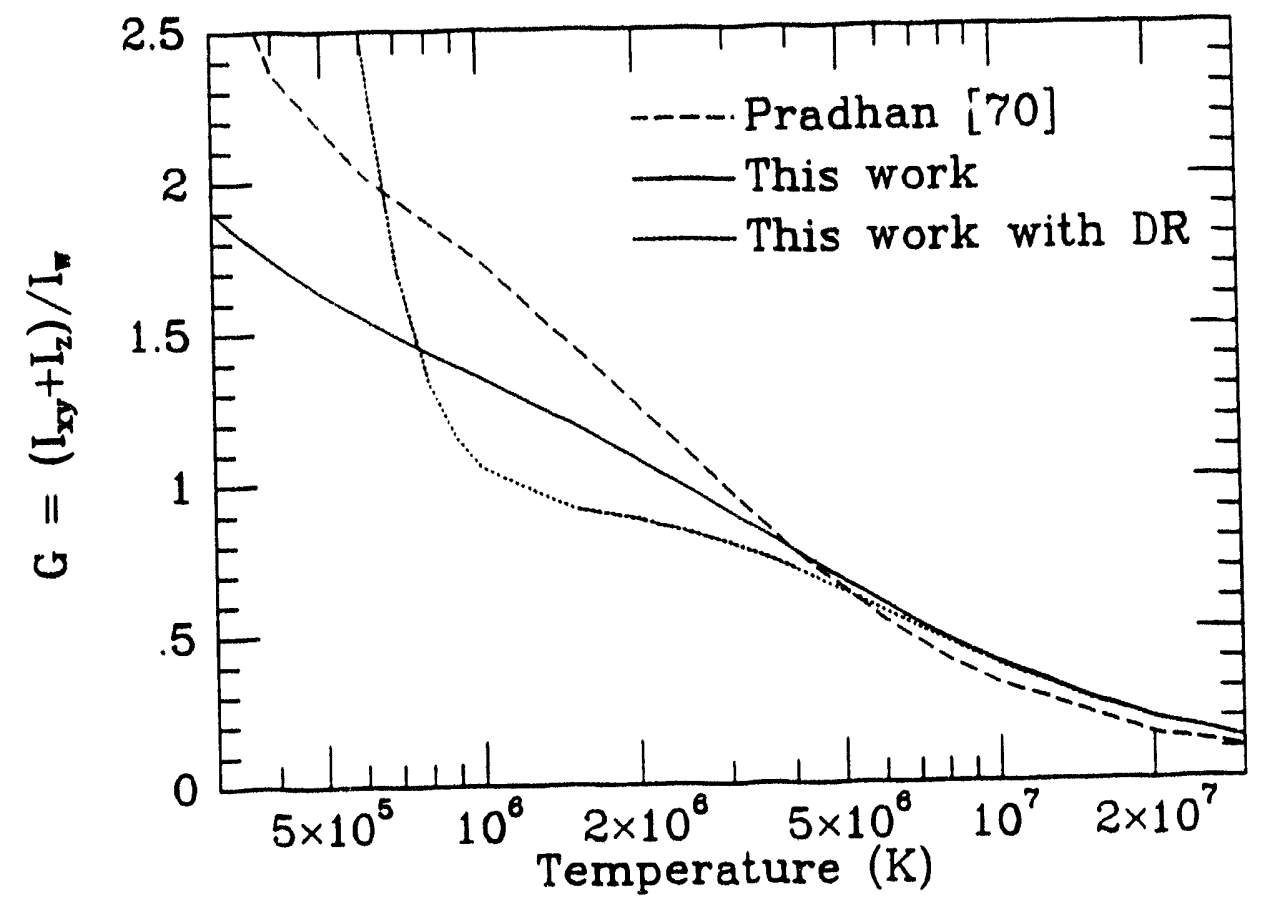

Figure 8.13: Comparison of experimental and theoretical results for temperaturesensitive line ratio $G$. Experimentally determined cross sections for $w, x y$, and $z$ were integrated over all energies weighted with a Maxwellian energy distribution to derive rate coefficients. 
past $10^{8} \mathrm{~K}$. Of course, these figures are only true for emission from He-like neon. At high temperatures such as $10^{8} \mathrm{~K}$, most neon ions will be fully stripped and the majority of He-like $K_{\alpha}$ emission will come from the recombination of $\mathrm{H}$-like ions.

For astrophysical applications, any complete model of He-like emission from collisional plasmas must include contributions from the RR and DR of $\mathrm{H}$-like ions, ISI of Li-like ions, and collisional transfer from $z$ to $x y$ caused by $\mathrm{H}$ and He nuclei. In photoionized nebulae, cross sections for photoexcitation, photoionization, $D R$, and RR are needed. Although great progress has been made in experimental and theoretical investigations of these atomic processes, much work remains to be done to ensure that plasma diagnostics are reliable and properly applied. 


\section{Chapter 9}

\section{Concluding Remarks}

The goal of this experimental program was to accurately and completely characterize all major atomic processes involved in the emission of $K_{\alpha}$ radiation from He-like neon, in order to establish a firm foundation for the development of astrophysical X-ray plasma diagnostics. Although it proved impossible to study the contribution of $\mathrm{H}$-like ions because recombination cross sections are so much smaller than those for direct excitation, virtually all relevant processes in He-like and Li-like ions were measured with an average relative uncertainty of approximately 10 or $15 \%$ over an electron energy range of 600 to $13,000 \mathrm{eV}$. Most theoretical predictions were validated, while several important discrepancies were also discovered. Virtually all of the measurements made were the first of their kind for He-like neon, and several were the first or one of the first ever made for any species of highly charged ion.

\subsection{Summary of Results}

Perhaps the most impressive result is the measurement of the radiative lifetime of the $1 s 2 s^{3} S_{1}$ state that produces line $z$, accurate to nearly $1 \%$. The lifetime of this metastable state is the cornerstone of the He-like neon density diagnostic, and the method developed here can be applied to measure the radiative rates of other transitions of astrophysical importance. The other parameter of importance to the density diagnostic, the cross section for collisional excitation of the ${ }^{3} S_{1}$ state to the ${ }^{3 P}$ states, was also studied. Although an absolute cross section could not be measured, as was the case for all the cross sections measured in this experiment, its energy dependence agreed well with theoretical predictions. This was probably the first 
measurement of a cross section for collisional excitation from a metastable state in a highly charged ion.

The measurement of $\mathrm{X}$-ray polarization, although not a complete success, was one of the very few $\mathrm{X}$-ray line polarization measurements ever made and confirmed theoretical predictions of the relative polarization of at least two emission lines. The study of innershell ionization in Li-like neon likewise confirmed general expectations while distinguishing between several theoretical predictions of the fluorescence yields of the Li-like satellites $q$ and $r$.

Although the EBIT electron beam lacked the energy resolution required to accurately determine the strengths of RE resonances, measurement of electron impact excitation cross sections showed that theory underestimates the relative cross sections for $x y$ and $z$ by an average of $20 \%$. There were also indications that high order resonant excitation peaks may be significantly stronger than predicted by theory. Dielectronic recombination satellites were also measured and resolved for the first time, yielding resonance strengths and very accurate wavelength measurements. Observed strengths of Li-like DR satellites showed significant disagreement with several theories, while measured strengths of Be-like satellites agreed very well with theoretical predictions, although the Be-like results could vary by almost a factor of two depending on which theoretical values for the fluorescence factor of line $q$ were used.

From an instrumental point of view, the experiment was also a success. Low energy operation of EBIT was shown to be entirely feasible, thus preparing the way for further experiments of interest to $\mathrm{X}$-ray astrophysicists. The low energy flat crystal spectrometer, with its modified proportional counter, proved to be an extremely versatile and effective instrument and has been used extensively in other EBIT experiments. 


\subsection{Future Work}

Examples of future astrophysically-directed research on EBIT include the study of He-like ions of other abundant elements such as oxygen, nitrogen, sulfur, and iron. Studies of carbon are probably not feasible because of the extremely low energies required. Another obvious subject for study on EBIT is iron L-shell emission. Efforts are now underway to replace the $4-\mu \mathrm{m}$ polypropylene detector window with a thinner and more transmissive polyimide window so that $\mathrm{X}$-rays of even lower energy (Fe $L$ shell down to $\sim 700 \mathrm{eV}$, He-like $\mathrm{O}$ at $570 \mathrm{eV}$ ) can be observed. To increase the overall efficiency of the low-energy spectrometer, it may be worthwhile to use grazing incidence reflection gratings in place of Bragg crystals for measurements that do not require very high energy resolution.

For the specific case of He-like neon measurements, several improvements are possible, although most require significant technological advances or investments of time and money. The two factors that most limited the accuracy of measurements in this experiment were the electron beam energy resolution and uncertainties in line polarization. Better energy resolution, to more precisely map out resonances and cascade jumps, requires that the space charge of the electron beam be reduced. This requires reducing the electron density which can most easily be done by running the beam at lower current. Lower density also means that there would be much less collisional transfer from $z$ to $x y$, and thus less uncertainty in the polarizaticns of those lines. A significant drawback is a large drop in $\mathrm{X}$-ray flux. Since density scales as current, and countrate scales as $I^{3 / 2}$, improving the energy resolution from $75 \mathrm{eV}$ to 20 $\mathrm{eV}$ means a drop of a factor of 7 in emitted flux. A new power supply would also be required to reduce the ripple in the drift tube voltage.

A large part of the uncertainty in polarization comes from the correction for transverse electron velocities in the beam (equation 6.25). One way to reduce the 
uncertainty in that correction is to measure the polarization of a highly polarized line with reliable theoretical polarization (such as $w$ ) very accurately and thus determine the extent of the depolarization. This requires extremely accurate calibration of crystal reflectivities, preferably using an intrinsically unpolarized line (which may be hard to find near $1 \mathrm{keV}$ ). Precise calibration is a tedious and time-consuming process, but one which is required for relative cross sections to be measured with accuracies of $5 \%$ or better.

It would also be desirable to build another spectrometer that could make simultaneous observations of Lyman- $\alpha$ and He-like $K_{\beta}\left(1 s^{3} p^{1} P_{1} \rightarrow 1 s^{2}{ }^{1} S_{0}\right)$. The intensity of Ly- $\alpha$ could be used to estimate the relative abundance of $\mathrm{H}$-like ions, while the relative intensities of $K_{\beta}$ and $w$ (or all $\mathrm{K}_{\alpha}$ lines) can be used as a temperature diagnostic. A second spectrometer could also be used to make polarization measurements (in conjunction with the first FCS) using either the "two crystal" method or the "vertical-horizontal" method.

Background emission from impuri trapped in EBIT is especially troublesome for low-energy measurements. li ... uuld be substantially reduced, solid state spectrometers, such as $\mathrm{Si}(\mathrm{Li})$ detectors, might be able to resolve some radiative recombination peaks. The RR peaks could then be used to normalize He-like $\mathrm{Ne} K_{\alpha}$ emission and thus derive (semi-)absolute cross sections. Of even greater benefit would be the ability to study just one ionic species at a time. EBIT scientists are currently studying the feasibility of superposing a radio-frequency signal on the drift tubes which could be tuned to match cyclotron resonances and expel all ions except the one species under stady. If successful, this capability would increase the scientific power of EBIT immensely. 


\section{References}

[1] L.W. Acton and W.A. Brown, Astrophys. J. 25, 1065 (1978).

[2] K. Alder and R. M. Steffen, in The Electromagnetic Interaction in Nuclear Spectroscopy, edited by W. D. Hamilton (American Elsevier, New York, 1975), Chap. 1, pp. 1-38; R. M. Steffen and K. Alder, ibid., Chap. 12, pp. 505-582.

[3] M. Arnaud and R. Rothenflug, Astron. Astrop. Suppl. Series 60, 425 (1985).

[4] A. Bar-Shalom, M. Klapische, and J. Oreg, Phys. Rev. A 38, 1773 (1988).

[5] J. R. Bednar, C. L. Cocke, B. Curnutte, and R. Randall, Phys. Rev. A 11, 460 (1975).

[6] P. Beiersciorfer and B.J. Wargelin, Rev. Sci. Instrum., accepted.

[7] P. Beiersdorfe: et al., Phys. Rev. A 46, 3812 (1992).

[8] P. Beiersdorfer, et al., Phys. Rev. A 46, R25 (1992).

[9] P. Beiersdorfer, et al., Proceedings of the XVII Int'l Conf. on the Physics of Electron and Atomic Collisions, Brisbane, Australia, July 10-16, 1991 (IOP Publishing, London, 1992), p. 313.

[10] P. Beiersdorfer, et al., Phys. Rev. Lett. 67, 2272 (1991).

[11] P. Beiersdorfer, et al., Rev. Sci. Instrum. 61, 2338 (1990).

[12] P. Beiersdorfer, et al., Phys. Rev. Lett. 65, 1995 (1990).

[13] M. Bitter, et al., Phys. Rev. A 32, 3011 (1985).

[14] M. Bitter, et al., Phys. Rev. Lett. 43, 129 (1979).

[15] G.R. Blumenthal, G.W.F. Drake, and W.H. Tucker, Astrophys. J. 172, 205 (1972).

[16] C.J. Borkowsi and M.K. Kopp, Rev. Sci. Instrum. 39, 155 (1968).

[17] J. P. Cassinelli and J. H. Swank, Astrophys. J. 271, 681 (1983).

[18] J. I. Castor, in Instabilities in Luminous Early Type Stars, eds. H. G. J. L. Lamers, C. W. H. de Loore (Reidel, Dordrecht, 1987), p. 159.

[19] S. Chantrenne, P. Beiersdorfer, R. Cauble, and M.B. Schneider, Phys. Rev. Lett. 69, 265 (1992).

[20] M. H. Chen and B. Crasemann, At. Data and Nucl. Data Tables 37, 420 (1987). 
[21] M. H. Chen, At. Data and Nucl. Data Tables 34, 301 (1986).

[22] M. H. Chen (private communication).

[23] M. H. Chen, Phys. Rev. A 31, 1449 (1985).

[24] C. L. Cocke, B. Curnutte, and R. Randall, Phys. Rev. Lett. 31, 507 (1973).

[25] C. L. Cocke, B. Curnutte, J. MacDonald, and R. Randall, Phys. Rev. A 9, 57 (1974).

[26] R. D. Cowan, The Theory of Atomic Structure and Spectra (U. of Calif. Press, Berkeley, CA, 1981).

[27] G. W. F. Drake, Can. J. Phys. 66, 586 (1988).

[28] G. W. F. Drake, Astrophys. J. 158, 1199 (1969); 163, 439 (E) (1971).

[29] G. W. F. Drake, Phys. Rev. A 3, 908 (1971).

[30] G. W. F. Drake and A. Dalgarno, Astrophys. J. 157, 459 (1969).

[31] R. W. Dunford, et al., Phys. Rev. A 41, 4109 (1990).

[31a] A. Fabian, in Proceedings of the Tenth International Colloquium on UV and $X$-ray Spectroscopy of Astrophysical and Laboratory Plasmas, ed. E. H. Silver and S. M. Kahn, (Cambridge University Press, Cambridge, 1993).

[32] A.H. Gabriel, Mon. Not. R. Astron. Soc. 160, 99 (1972).

[33] A. H. Gabriel and C. Jordan, Nature 221, 947 (1969).

[34] A. H. Gabriel and C. Jordan, Mon. Not. R. Astron. Soc. 145, 241 (1969).

[35] H. Gould, R. Marrus, and R. W. Schmieder, Phys. Rev. Lett. 31, 504 (1973).

[36] H. Gould and R. Marrus, Bull. Am. Phys. Soc. 21, 84 (A) (1976).

[37] H. Gould, R. Marrus, and P. J. Mohr, Phys. Rev. Lett. 33, 676 (1974).

[38] E. Gullikson (private communication).

[39] J. R. Henderson, et al., Phys. Rev. Lett. 65, 705 (1990).

[40] B. L. Henke, E. M. Gullikson, and J. C. Davis, At. Data and Nucl. Dota Tables, submitted.

[41] G. Herrmann, J. Appl. Phys. 29, 127 (1958).

[42] G. Hurbricht and E. Träbert, Z. Phys. D 7, 243 (1987).

[43] M. K. Inal and J. Dubau, J. Phys. B 22, 3329 (1989). 
[44] M. K. Inal and J. Dubau, J. Phys. B 20, 4221 (1987).

[45] W. R. Johnson and C. Lin, Phys. Rev. A 9, 1486 (1974).

[46] C. Jordan and N.J. Veck, Sol. Phys. 78, 125 (1982).

[47] E. Källne, J. Källne, and A.K. Pradhan, Phys. Rev. A 28, 467 (1983).

[48] F.P. Keenan, et al., Phys. Rev. A 39, 4092 (1989).

[49] D.A. Knapp, et al., Phys. Rev. A 47, 2039 (1993).

[50] D.A. Knapp, et al., Phys. Rev. Lett. 62, 2104 (1989).

[51] R. D. Knight and M. H. Prior, Phys. Rev. A 21, 179 (1980).

[52] M. A. Levine, et al., Phys. Scripta T22, 157 (1988).

[53] M. A. Levine, R.E. Marrs, and R.W. Schmieder, Nucl. Instrum. Methods A237, 429 (1985).

[54] D. A. Liedahl, et al., in Atomic Processes in Plasmas, Proceedings of the DAMOP Workshop, ed., E. Marmar and J. Terry (American Institute of Physics, 1992).

[55] D. A. Liedahl, et al., Astrophys. J. 391, 306 (1992).

[56] W. Lotz, Zeitschrift für Physik 202, 205 (1967).

[57] J. Mandel, The Statistical Analysis of Experimental Data (Dover, New York, 1964), pp. 295-303.

[58] R. Marrus, et al., Phys. Rev. A 39, 3725 (1989).

[59] D. L. McKenzie, Astrophys. J. 322, 512 (1987).

[60] D. L. McKenzie and P.B. Landecker, Astrophys. J. 259, 372 (1982).

[61] R. Mewe, Astron. Astrophys. Review 25, 7 (1991).

[62] R. Mewe and J. Schrijver, Astron. Astrophys. 65, 99 (1978).

[63] R. Mewe and J. Schrijver, Astron. Astrophys. 87, 55 (1978).

[64] H. W. Moos and J. R. Woodworth, Phys. Rev. Lett. 30, 775 (1973).

[65] J. Nilsen, At. Data and Nucl. Data Tables 38, 339 (1988).

[66] A.L. Osterheld (private communication).

[67] R. Pallavicini, et al., Astrophys. J. 248, 279 (1981). 
[68] A. K. Pradhan, Astrophys. J. 263, 477 (1982).

[69] A. K. Pradhan, D. W. Norcross, and D. G. Hummer, Astrophys. J. 246, 1031 (1981).

[70] A. K. Pradhan, D. W. Norcross, and D. G. Hummer, Phys. Rev. A 23, 619 (1981).

[71] A. K. Pradhan and J. M. Shull, Astrophys. J. 249, 821 (1981).

[72] K. Reed (private communication).

[73] G. B. Rybicki, in Instabilities in Luminous Early Type Stars, eds. H. G. J. L. Lamers, C. W. H. de Loore (Reidel, Dordrecht, 1987), p. 175.

[74] D. H. Sampson and H. L. Zhang (private communication).

[75] M. B. Schneider, et al., Phys. Rev. A 45, R1291 (1992).

[76] M. B. Schneider, et al., Int'l Symp. on Electron Beam Ion Sources and Their Applications, ed. A. Hershcovitch, AIP Conf. Proc. No. 188 (AIP, New York, 1989), p. 158.

[77] M. B. Schneider, et al., in Proc. Int. Symp. on Electron Beam Ion Sources and their Applications, edited by A. Hershcovitch, AIP Conf. Proc. No. 188 (AIP, New York, 1989) p. 145.

[78] TFR group, J. G. Doyle, and J. L. Schwob, J. Phys. B 15, 817 (1982).

[79] L. A. Vainshtein and U. I. Safronova, At. Data and Nucl. Data Tables 21, 49 (1978).

[80] D. A. Vogel, Ph.D. thesis, Georgia Institute of Technology, 1992.

[81] D. A. Vogel, et al., Z. Phys. D 21, S193 (1991).

[82] S. D. Vrtilek, et al., Astrophys. J. Suppl. Ser. 76, 1127 (1991).

[83] B. J. Wargelin, P. Beiersdorfer, and S. M. Kahn, Phys. Rev. Lett. 71, 2196 (1993).

[84] C. J. Wolfson, et al., Astrophys. J. 269, 319 (1983).

[85] K. Wong, et al., Z Phys. D 21, S197 (1991).

[86] J. R. Woodworth and H. W. Moos, Phys. Rev. A 12, 2455 (1975).

[87] H. L. Zhang, D. H. Sampson, and R. E. H. Clark, Phys. Rev. A 41, 198 (1990). 
11
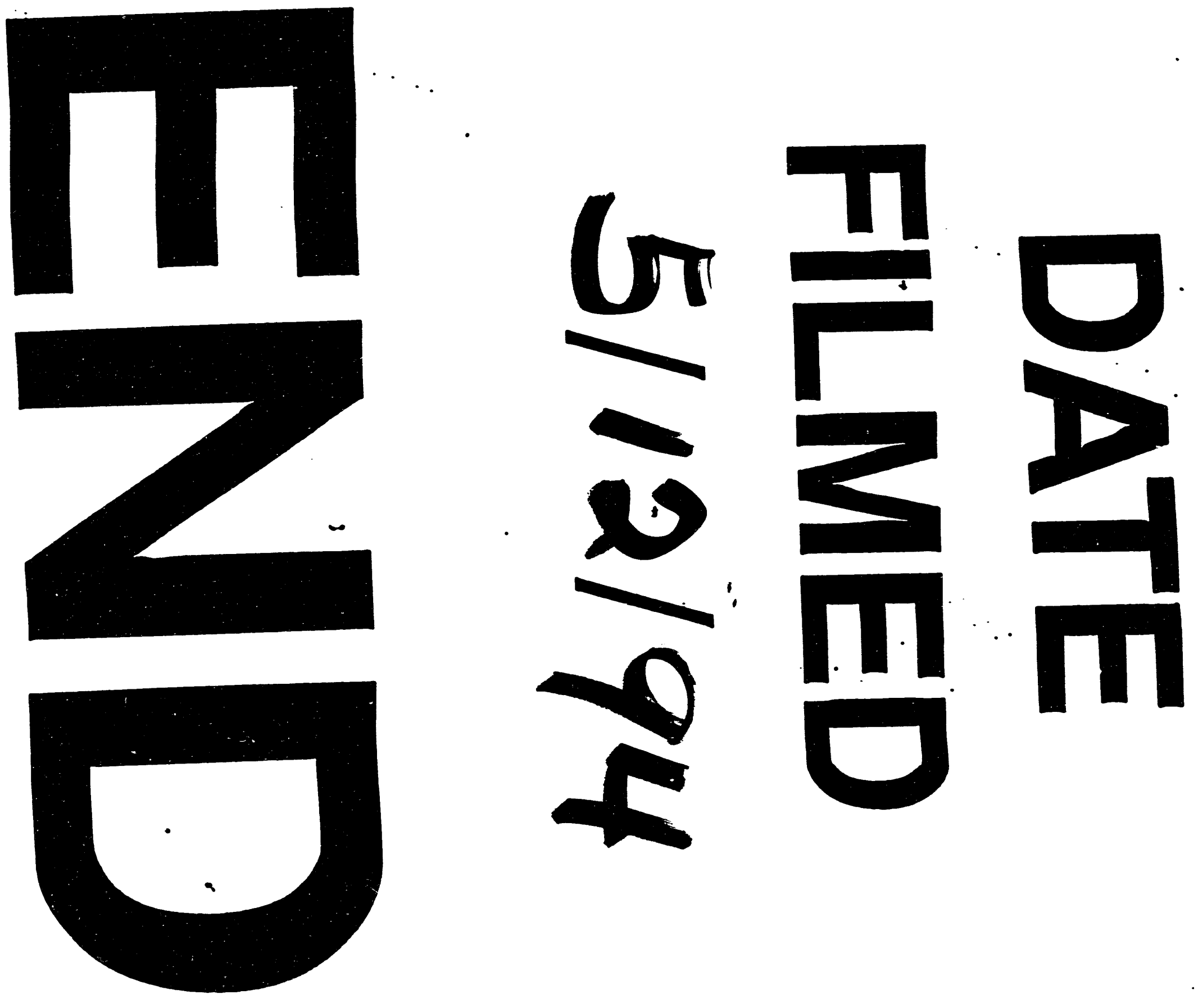
